\title{
Sequence of segmental contributions in the lower cervical spine and their application to cervical arthroplasty
}

Citation for published version (APA):

Boselie, T. F. M. (2019). Sequence of segmental contributions in the lower cervical spine and their application to cervical arthroplasty. [Doctoral Thesis, Maastricht University]. ProefschriftMaken Maastricht. https://doi.org/10.26481/dis.20190208ab

Document status and date:

Published: 01/01/2019

DOI:

10.26481/dis.20190208ab

Document Version:

Publisher's PDF, also known as Version of record

\section{Please check the document version of this publication:}

- A submitted manuscript is the version of the article upon submission and before peer-review. There can be important differences between the submitted version and the official published version of record. People interested in the research are advised to contact the author for the final version of the publication, or visit the DOI to the publisher's website.

- The final author version and the galley proof are versions of the publication after peer review.

- The final published version features the final layout of the paper including the volume, issue and page numbers.

Link to publication

\footnotetext{
General rights rights.

- You may freely distribute the URL identifying the publication in the public portal. please follow below link for the End User Agreement:

www.umlib.nl/taverne-license

Take down policy

If you believe that this document breaches copyright please contact us at:

repository@maastrichtuniversity.nl

providing details and we will investigate your claim.
}

Copyright and moral rights for the publications made accessible in the public portal are retained by the authors and/or other copyright owners and it is a condition of accessing publications that users recognise and abide by the legal requirements associated with these

- Users may download and print one copy of any publication from the public portal for the purpose of private study or research.

- You may not further distribute the material or use it for any profit-making activity or commercial gain

If the publication is distributed under the terms of Article $25 \mathrm{fa}$ of the Dutch Copyright Act, indicated by the "Taverne" license above, 
Sequence of segmental contributions in the lower cervical spine and their application to cervical arthroplasty

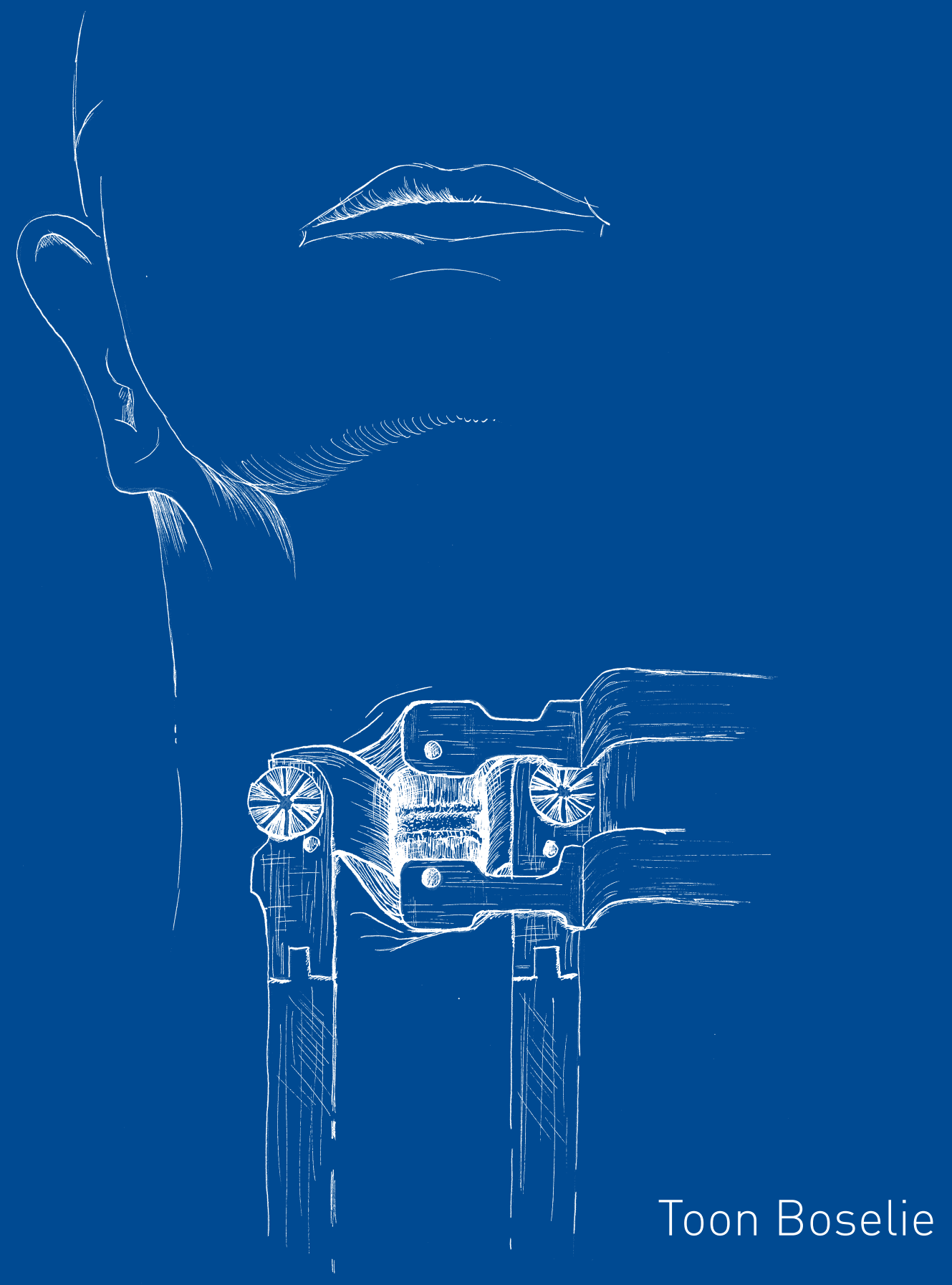





\title{
Sequence of segmental contributions in the lower cervical spine and their application to cervical arthroplasty
}

\author{
Toon F.M. Boselie
}


(C) copyright Toon Boselie, Maastricht 2018

Cover illustration Lindi Bronneberg-Jacobs

Printing: ProefschriftMaken || www.proefschriftmaken.nl

ISBN 978-94-6380-184-3

All rights reserved. No part of this publication may be reproduced, stored in a retrieval system or transmitted, in any form or by any means, electronic, mechanical, photocopying, recording or otherwise, without prior permission of the author or the copyright-owning journals for previous published chapters. 


\title{
Sequence of segmental contributions in the lower cervical spine and their application to cervical arthroplasty
}

\author{
PROEFSCHRIFT \\ ter verkrijging van de graad van doctor aan de Universiteit Maastricht \\ op gezag van de Rector Magnificus, Prof. dr. Rianne M. Letschert, \\ volgens het besluit van het College van Decanen, \\ in het openbaar te verdedigen \\ op vrijdag 8 februari 2019 om 12.00 uur
}

door

Antonius (Toon) Franciscus Maria Boselie 


\section{Promotor}

Prof. dr. R. A. de Bie

\section{Co-promotor}

Dr. H. van Santbrink

\section{Beoordelingscommissie}

Prof. dr. L. van Rhijn (voorzitter)

Dr. L. Jacobi-Postma

Prof. dr. W. Peul (Leids Universitair Medisch Centrum)

Prof. dr. Y. Temel

Dr. A. Vilanova (TU Delft) 


\section{Table of Contents}

Chapter 1 General introduction and thesis outline

Chapter 2 Arthroplasty versus Fusion in Single-Level Cervical Degenerative Disc Disease

Chapter 3 Cervical Vertebrae Tracking in Video-Fluoroscopy Using the Normalized Gradient Field

Chapter 4 A pilot study of sequence of segmental contributions in the lower cervical spine during active extension and flexion: healthy controls vs. cervical degenerative disc disease patients

Chapter 5 Cervical spine kinematics after anterior cervical discectomy with or without implantation of a mobile cervical disc prosthesis; an RCT

Chapter 6 Sequence of segmental contributions in the caudal cervical spine in arthroplasty and ACD patients: a randomized controlled trial

Chapter 7 General discussion

Valorisation

Summary

List of abbreviations

Dankwoord

Curriculum Vitae

List of Publications 

Chapter

General introduction and thesis outline 



\section{Cervical degenerative disc disease}

Cervical degenerative disc disease (CDDD) is degeneration of a cervical intervertebral disc and/or the adjoining vertebral bodies. Possible sequelae are a bulging or herniated intervertebral disc, foraminal narrowing due to loss of disc space height or osteophyte formation, or a combination of these. A common resulting clinical symptom is cervical radiculopathy. Other clinical symptoms that can be caused are axial pain, sensory loss or motor weakness due to spinal cord compression, and headache [1-4].

The mean annual incidence of cervical radiculopathy is reported to be 83 per 100,000 people, with a peak incidence of 202 per 100,000 for people aged 50 to 54 years. After the age of 60 years, a rapid decline in incidence is described, more dramatic in females than in males $[5,6]$.

Treatment of cervical radiculopathy can be non-surgical or surgical. The majority of patients respond well to non-surgical treatment options, such as physical therapy, use of a collar, analgesic or anti-inflammatory medication [7]. With non-surgical treatment, up to $60 \%$ of patients are satisfied or very satisfied at six weeks after start of treatment [8]; up to $83 \%$ of the patients who undergo conservative treatment report good or excellent results at a mean follow-up of two years [9]. Surgical treatment can be considered in cases of insufficient relief of symptoms with these non-surgical treatment options [1013]. In case of radiculopathy, the primary goal of surgical intervention is to relieve radiating arm pain, as any neurological deficit that is present may be permanent despite treatment. The reported proportion of patients who are eventually treated surgically ranges from $8 \%$ to $35 \%[6,9,14]$.

\section{Surgical treatment of cervical radiculopathy due to CDDD}

The most commonly performed procedure is anterior cervical discectomy with fusion (ACDF). Also used is anterior cervical discectomy without fusion promoting actions (ACD). In ACD, all or part of an intervertebral disc is removed [15]. In ACDF, a bony fusion between the two vertebrae is then promoted by interposition of a cage, autograft, or allograft material in the disc space after the discectomy. Anterior plate and screw fixation can be added. In ACD the rate of fusion in the operated segment is already up to $80 \%$. The additional fusion promoting actions in ACDF increase the rate of fusion to over $95 \%$ $[3,11,16,17]$.

Fusion and simple discectomy both have good clinical results, with short term (at six to eight weeks) satisfactory or good results in $90 \%$ to $100 \%$ of the patients $[16,18-20]$. In the long term, patient satisfaction slowly drops to 68-96\% after 7-20 years due to recurrent symptoms caused by CDDD at an adjacent level $[11,19,21]$. This is called adjacent segment disease (ASDis). There is insufficient evidence to adequately compare non-sur- 
gical and surgical treatment. In a systematic review of trials comparing non-surgical treatment with surgical intervention for CDDD the authors concluded that there was low quality evidence (only 1 trial, $n=81$ ) that surgery relieved pain faster than non-surgical therapy. At one year there no longer was a significant difference between the two groups [22]. Long term outcomes for surgical and non-surgical treatment cannot be compared as there is no prospective long term data on non-surgical treatment of CDDD. Therefore, the rate of ASDis, as well as long term patient satisfaction are not known in the nonsurgical treatment group.

\section{Adjacent segment degeneration and adjacent segment disease}

Adjacent segment degeneration is the occurrence of CDDD at a level adjacent to a previously treated segment. There can be purely radiological changes, in which case the term adjacent segment degeneration (ASDeg) is used. When the degeneration leads to new symptoms, such as a radiculopathy or myelopathy, the term adjacent segment disease (ASDis) is used $[23,24]$. The reported incidence for ASDis is $2.9 \%$ per year, or $25 \%$ after ten years [23].

The underlying mechanism is thought to be compensation of the loss of motion in the fused segment, resulting in overstraining of the adjacent segments. There is no in vivo proof on this subject, the principle was only shown in cadaver studies. In a porcine cadaver study, Eck et al reported an increase in intradiscal pressure in an intervertebral disc adjacent to a fused segment [25]. The same study also reported increased mobility in the segments adjacent to a fused segment, although only significant in the cranial adjacent segment [25]. An increase in adjacent segment ROM was reported in a small nonrandomized study $(n=25)$ [26]. However, in a small subgroup of patients $(n=22)$ that were enrolled in the Bryan FDA IDE trial no increase in adjacent segment ROM was seen. In the latter study an increase in anterior-posterior translation was seen in the ACDF group, which was significant at 6 months, but not at 12 months after surgery [27].

There is much debate about the role of fusion in the development of ASDis [28-30]. The degeneration might also be part of the natural history, with continuing progression in a degenerating spine. One study that is cited as an example for this hypothesis, was published by Herkowitz et al. in 1990, in which the rate of ASDeg was compared between a group that underwent posterior foraminotomy and a group that underwent ACDF [31]. In this study, a higher rate of ASDeg was reported for the group that underwent posterior foraminotomy, a surgical procedure that rarely results in fusion. Although there is much debate about ASDeg/ASDis, cervical arthroplasty was introduced. 


\section{Cervical arthroplasty}

Cervical disc prostheses were developed in an effort to reduce the incidence of ASDis by preserving motion in the operated segment [32]. These movable prostheses can be placed in the intervertebral space after the discectomy. Motion preservation is thought to reduce aforementioned sequelae in adjacent motion segments, which in turn could lead to less ASDeg/ASDis. Given the relatively low annual incidence of ASDeg/ASDis, long term studies are necessary to prove or disprove this protective effect.

The goal of CA should not only be to preserve mobility, but to facilitate normal movement, as abnormal mobility can intuitively still lead to increased degeneration. Since the introduction of cervical disc prostheses in the 1980s, several types have been developed, with different materials and movement characteristics [29, 33, 34].

\section{Motion analysis}

A definition of normal motion is necessary to be able to determine if a cervical disc prosthesis facilitates normal motion. The most commonly used method to study motion after $\mathrm{CA}$ is the use of segmental intervertebral range of motion measurements (sROM). These are measured in the sagittal plane. There are several methods of measuring the sROM. Three commonly referred methods are the methods of Bakke, Buetti-Bäuml, and Penning. These methods differ in the landmarks that are used, and the handling of the images.

The method of Penning is the most reliable of these three methods [35]. 

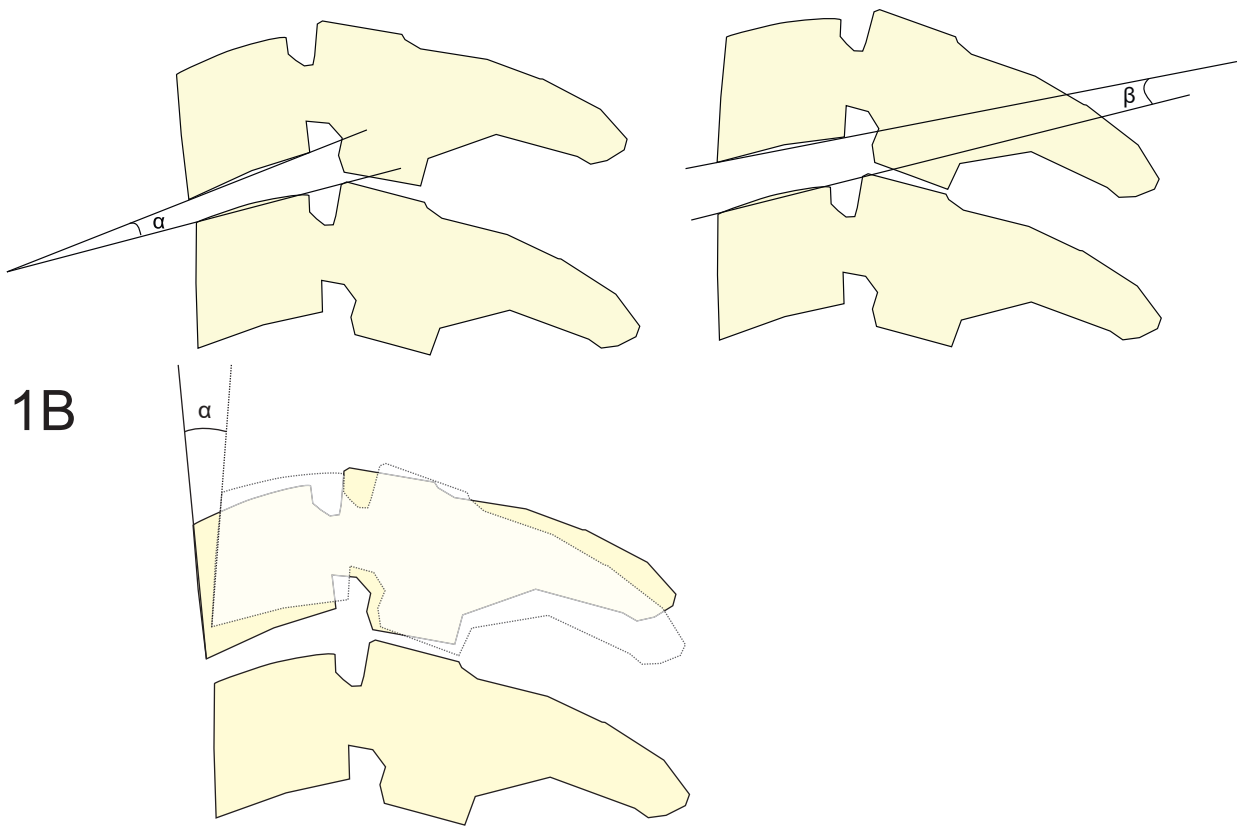

Figure 1A: method of Bakke. Two lines are drawn, parallel to the superior and inferior endplates of a disc space, on function radiographs. The angle of the disc space is therefore known in a maximum flexion position $(\alpha)$ and in the maximum extension position ( $\beta$ ), the difference is the sROM for that motion segment.

Figure 1B: method of Penning. The two images are superimposed, the contours of the caudal vertebra are then matched on both images. The angle between the two contours of the cranial vertebra is the sROM for that motion segment.

The method is easy and quick. The main downside of the use of sROMs is their high intraand interindividual variability [36-40]. Therefore, the normal ranges that have been reported in healthy subjects are very wide (Table 1). Differences of up to 10 degrees within a single motion segment in the same patient are possible at different time points [40]. The high variability makes the method useless in individual subjects, and only offers limited use in larger groups.

Table 1: Average sROM and their respective SD for motion segments C4-C5, C5-C6, and C6-C7 for reported populations of healthy controls. Normal range is often defined as mean +/- 2SD. (from: Bogduk, N. and S. Mercer, Biomechanics of the cervical spine. I: Normal kinematics. Clin Biomech (Bristol, Avon), 2000. 15(9): p. 63348) [41].

\begin{tabular}{l|c|c|c|} 
& \multicolumn{3}{|c|}{ Mean sROM C, SD) } \\
\hline Literature & C4-C5 & C5-C6 & C6-C7 \\
\hline Aho et $a l^{10}$ & $22(4)$ & $28(4)$ & $15(4)$ \\
\hline Bhalla et al II $^{11}$ & $23(1)$ & $19(1)$ & $18(3)$ \\
\hline Lind et al $^{12}$ & $16(6)$ & $15(8)$ & $11(7)$ \\
\hline Dvorak et al & $19(4)$ & $20(4)$ & $19(4)$ \\
\hline
\end{tabular}


Alternatively, the sequence of segmental contributions to flexion and/or extension of the entire cervical spine has been proposed as a more consistent parameter of cervical spine motion $[41,42]$. For this type of analysis a fluoroscopic recording of a subject performing a maximum extension to full bending flexion (or vice versa) is necessary. Throughout the flexion or extension movement of the entire cervical spine, the various motion segments are contributing at different moments. The sequence was found to be fairly consistent, with a biphasic motion pattern in the segments within block $\mathrm{C} 4-\mathrm{C} 7$, with a caudal to cranial sequence of contributions during the first phase, and a cranial to caudal sequence during the second phase. A biphasic pattern was also described during extension, with a first phase that in many cases did not show a specific sequence, while the second phase consisted of a cranial to caudal sequence of segmental contributions in 9 out of 10 subjects.

The consistent sequences that were reported during flexion and extension were the reason to choose the second method, the analysis of the sequence of segmental contributions, as the primary focus to investigate cervical spine motion in relation to cervical arthroplasty.

\section{Aim of this thesis}

The primary goal of this thesis is to investigate if cervical arthroplasty restores normal motion. To this end a computer assisted method of analysis was developed. If normal motion is restored, the premise on which the assumed reduction in ASDeg/ASDis is based is met. Secondly, the aim is to compare clinical results of arthroplasty and fusion.

\section{Outline of the thesis}

In chapter 2, a systematic review comparing the clinical and radiological results of arthroplasty versus fusion was performed. Due to the length of follow up in the available studies at the time of reviewing, the maximum follow up was 2 years.

In chapter 3, a novel method of tracking vertebrae throughout all frames of an x-ray cinematographic recording is described. This method was designed to track rotations of the skull and all cervical vertebrae in recordings of a subject performing a full extension to 'bending' flexion movement (flexion cinematographic recording, FCR), and vice versa (extension cinematographic recording, ECR). This method uses a normalised gradient field to compare the orientation of a user defined template area between frames.

In chapter 4, the aforementioned method of tracking vertebrae is used to determine the sequence of segmental contributions of the segments C4-C5, C5-C6 and C6-C7 to flexion and extension of the caudal cervical spine (C4-C7). A definition of 'normal' motion was 
made, based on ECR data in healthy controls. The method was then also applied to data on preoperative CDDD patients to determine reproducibility, sensitivity, and specificity of this definition in distinguishing between healthy controls and CDDD patients.

In chapter 5 , the protocol for a randomized controlled trial (RCT) to investigate cervical spine mobility and clinical outcome after simple discectomy or arthroplasty for radiculopathy due to single level CDDD is described. In this RCT patients were evaluated at three time points. During each evaluation an FCR and ECR were recorded. Additionally, patients were required to fill in several questionnaires, and underwent a neurological examination.

In chapter 6 , the results from the RCT are reported and the findings are discussed.

In chapter 7, the cumulative findings of the previous chapters and their applicability to daily practice are discussed. Suggestions for future research are made, based on this discussion. 


\section{References}

1. Persson, L.C., et al., Cervical radiculopathy: pain, muscle weakness and sensory loss in patients with cervical radiculopathy treated with surgery, physiotherapy or cervical collar. A prospective, controlled study. Eur Spine J, 1997. 6(4): p. 256-66.

2. Persson, L.C., J.Y. Carlsson, and L. Anderberg, Headache in patients with cervical radiculopathy: a prospective study with selective nerve root blocks in 275 patients. Eur Spine J, 2007. 16(7): p. 953-9.

3. Grob, D., Surgery in the degenerative cervical spine. Spine (Phila Pa 1976), 1998. 23(24): p. $2674-83$.

4. Polston, D.W., Cervical radiculopathy. Neurol Clin, 2007. 25(2): p. 373-85.

5. Salemi, G., et al., Prevalence of cervical spondylotic radiculopathy: a door-to-door survey in a Sicilian municipality. Acta Neurol Scand, 1996. 93(2-3): p. 184-8.

6. Radhakrishnan, K., et al., Epidemiology of cervical radiculopathy. A population-based study from Rochester, Minnesota, 1976 through 1990. Brain, 1994. 117 ( Pt 2): p. 325-35.

7. Kuijper, B., et al., Degenerative cervical radiculopathy: diagnosis and conservative treatment. A review. Eur J Neurol, 2009. 16(1): p. 15-20.

8. Kuijper, B., et al., Cervical collar or physiotherapy versus wait and see policy for recent onset cervical radiculopathy: randomised trial. BMJ, 2009. 339: p. b3883.

9. Saal, J.S., J.A. Saal, and E.F. Yurth, Nonoperative management of herniated cervical intervertebral disc with radiculopathy. Spine (Phila Pa 1976), 1996. 21(16): p. 1877-83.

10. Gore, D.R. and S.B. Sepic, Anterior cervical fusion for degenerated or protruded discs. A review of one hundred forty-six patients. Spine (Phila Pa 1976), 1984. 9(7): p. 667-71.

11. Gore, D.R. and S.B. Sepic, Anterior discectomy and fusion for painful cervical disc disease. A report of 50 patients with an average follow-up of 21 years. Spine (Phila Pa 1976), 1998. 23(19): p. 2047-51.

12. Klein, G.R., A.R. Vaccaro, and T.J. Albert, Health outcome assessment before and after anterior cervical discectomy and fusion for radiculopathy: a prospective analysis. Spine (Phila Pa 1976), 2000. 25(7): p. 8013.

13. White, A.A., 3rd, et al., Relief of pain by anterior cervical-spine fusion for spondylosis. A report of sixty-five patients. J Bone Joint Surg Am, 1973. 55(3): p. 525-34.

14. Wolff, M.W. and L.A. Levine, Cervical radiculopathies: conservative approaches to management. Phys Med Rehabil Clin N Am, 2002. 13(3): p. 589-608, vii.

15. Jacobs, W., et al., Single or double-level anterior interbody fusion techniques for cervical degenerative disc disease. Cochrane Database Syst Rev, 2011(1): p. CD004958.

16. Dowd, G.C. and F.P. Wirth, Anterior cervical discectomy: is fusion necessary? J Neurosurg, 1999. 90(1 Suppl): p. 8-12.

17. Fountas, K.N., et al., Anterior cervical discectomy and fusion associated complications. Spine (Phila Pa 1976), 2007. 32(21): p. 2310-7.

18. Donk, R.D., et al., What's the best surgical treatment for patients with cervical radiculopathy due to singlelevel degenerative disease? A randomized controlled trial. PLoS One, 2017. 12(8): p. e0183603.

19. Nandoe Tewarie, R.D., R.H. Bartels, and W.C. Peul, Long-term outcome after anterior cervical discectomy without fusion. Eur Spine J, 2007. 16(9): p. 1411-6.

20. Wirth, F.P., et al., Cervical discectomy. A prospective analysis of three operative techniques. Surg Neurol, 2000. 53(4): p. 340-6; discussion 346-8.

21. Palma, L., et al., Favourable long-term clinical outcome after anterior cervical discectomy. A study on a series of 125 patients undergoing surgery a mean of 11 years earlier. Acta Neurochir (Wien), 2010. 152(7): p. 1145-52.

22. Nikolaidis, I., et al., Surgery for cervical radiculopathy or myelopathy. Cochrane Database Syst Rev, 2010(1): p. CD001466.

23. Hilibrand, A.S., et al., Radiculopathy and myelopathy at segments adjacent to the site of a previous anterior cervical arthrodesis. J Bone Joint Surg Am, 1999. 81(4): p. 519-28.

24. Gore, D.R., et al., Neck pain: a long-term follow-up of 205 patients. Spine (Phila Pa 1976), 1987. 12(1): p. 1-5. 


\section{Chapter 1}

25. Eck, J.C., et al., Biomechanical study on the effect of cervical spine fusion on adjacent-level intradiscal pressure and segmental motion. Spine (Phila Pa 1976), 2002. 27(22): p. 2431-4.

26. Wigfield, C., et al., Influence of an artificial cervical joint compared with fusion on adjacent-level motion in the treatment of degenerative cervical disc disease. J Neurosurg, 2002. 96(1 Suppl): p. 17-21.

27. Sasso, R.C. and N.M. Best, Cervical kinematics after fusion and bryan disc arthroplasty. Journal of spinal disorders \& techniques, 2008. 21(1) :(pp 19-22), 2008. Date of Publication: Feb 2008.): p. 22.

28. Helgeson, M.D., A.J. Bevevino, and A.S. Hilibrand, Update on the evidence for adjacent segment degeneration and disease. Spine J, 2013. 13(3): p. 342-51.

29. Seo, M. and D. Choi, Adjacent segment disease after fusion for cervical spondylosis; myth or reality? Br J Neurosurg, 2008. 22(2): p. 195-9.

30. Rosenthal, P. and K.D. Kim, Cervical adjacent segment pathology following fusion: Is it due to fusion? World J Orthop, 2013. 4(3): p. 112-3.

31. Herkowitz, H.N., L.T. Kurz, and D.P. Overholt, Surgical management of cervical soft disc herniation. A comparison between the anterior and posterior approach. Spine (Phila Pa 1976), 1990. 15(10): p. 1026-30.

32. Goffin, J., et al., Intermediate follow-up after treatment of degenerative disc disease with the Bryan Cervical Disc Prosthesis: single-level and bi-level. Spine (Phila Pa 1976), 2003. 28(24): p. 2673-8.

33. Cummins, B.H., J.T. Robertson, and S.S. Gill, Surgical experience with an implanted artificial cervical joint. J Neurosurg, 1998. 88(6): p. 943-8.

34. Galbusera, F., et al., Biomechanical studies on cervical total disc arthroplasty: a literature review. Clin Biomech (Bristol, Avon), 2008. 23(9): p. 1095-104.

35. Osterhouse, M.D., et al., Reliability study of the Penning method for cervical intersegmental motion assessment: A pilot study. Vol. 10. 2002. 52-58.

36. Lind, B., et al., Normal range of motion of the cervical spine. Arch Phys Med Rehabil, 1989. 70(9): p. 692-5.

37. Dvorak, J., et al., In vivo flexion/extension of the normal cervical spine. J Orthop Res, 1991. 9(6): p. 828-34.

38. Bhalla, S.K. and E.H. Simmons, Normal ranges of intervertebral-joint motion of the cervical spine. Can J Surg, 1969. 12(2): p. 181-7.

39. Aho, A., O. Vartiainen, and O. Salo, Segmentary antero-posterior mobility of the cervical spine. Ann Med Intern Fenn, 1955. 44(4): p. 287-99.

40. Van Mameren, $H_{\text {., }}$ et al., Cervical spine motion in the sagittal plane (I) range of motion of actually performed movements, an X-ray cinematographic study. Eur J Morphol, 1990. 28(1): p. 47-68.

41. Bogduk, N. and S. Mercer, Biomechanics of the cervical spine. I: Normal kinematics. Clin Biomech (Bristol, Avon), 2000. 15(9): p. 633-48.

42. Van Mameren, H., Motion patterns in the cervical spine. 1988, Maastricht University. 


\section{Chapter}

\section{Arthroplasty versus Fusion in Single-Level Cervical Degenerative Disc Disease}

\section{A Cochrane Review}

Toon F. M. Boselie, Paul C. Willems, Henk van Mameren, Rob A. de Bie, Edward C. Benzel, Henk van Santbrink

Spine 2012

(adapted) 


\section{Abstract}

Study design A systematic review of randomized controlled trials (RCTs).

Objective To assess the effects of arthroplasty versus fusion in the treatment of radiculopathy or myelopathy, or both, due to single level cervical degenerative disc disease.

Summary of background data There is ongoing debate about whether fusion or arthroplasty is superior in the treatment of single-level cervical degenerative disc disease. Mainly because the intended advantage of arthroplasty compared with fusion, prevention of symptoms due to adjacent segment degeneration in the long term, is not confirmed yet. Until sufficient long-term results become available, it is important to know whether results of 1 of the 2 treatments are superior to the other in the first 1 to 2 years.

Methods We searched electronic databases for randomized controlled trials. We included randomized controlled trials that directly compared any type of cervical fusion with any type of cervical arthroplasty, with at least 1 year of follow-up. Study selection was performed independently by 3 review authors, and "risk of bias" assessment and data extraction were independently performed by 2 review authors. In case of missing data, we contacted the study authors or the study sponsor. We assessed the quality of evidence.

Results Nine studies (2400 participants) were included in this review; 5 of these studies had a low risk of bias. Results for the arthroplasty group were better than the fusion group for all primary comparisons, often statistically significant. For none of the primary outcomes was a clinically relevant difference in effect size shown. Quality of the evidence was low to moderate.

Conclusion There is low to moderate quality evidence that results are consistently in favor of arthroplasty, often statistically significant. However, differences in effect size were invariably small and not clinically relevant for all primary outcomes. 


\section{Introduction}

In the treatment of cervical degenerative disc disease surgery can be considered in cases of insufficient relief of symptoms with conservative treatment [1-5]. We focus on surgical intervention in case of radiculopathy, myelopathy, or a combination. Primary goals of surgical intervention are to relieve radiating arm pain in case of radiculopathy and to prevent progression of neurological deficit in case of myelopathy.

The most commonly performed cervical spine procedure is discectomy, either with or without fusion (for the rest of this review, these will be referred to as fusion and simple discectomy, respectively). Posterior laminoforaminotomy is less common and falls outside the scope of this review [6]. Fusion and simple discectomy have good clinical results [7-9]. For both types of surgery, a high rate of fusion, up to $80 \%$ in simple discectomy and more than $95 \%$ in fusion, has been reported $[2,7,10-12]$. Currently, there is much debate about whether fusion is a contributing factor to long-term clinical outcome and whether it causes accelerated degeneration of adjacent segments. If such adjacent disc degeneration causes symptoms, it is referred to as adjacent segment disease (ASDis). ASDis may be the result of increased intradiscal pressure and increased mobility at the segments adjacent to a fused segment [13]. However, it may simply be the result of natural progression of degeneration in an aging spine. Therefore, it remains unclear whether fusion should be regarded as a goal of treatment, should be prevented, or should be regarded as an accepted side effect of surgery [14]. The yearly risk of ASDis is calculated to be around $2.9 \%$. About two-thirds of these cases result in additional surgery $[1,2,8,15]$. To avoid ASDis as a presumed long-term effect of fusion, simple discectomy with implantation of a mobile disc prosthesis (for the rest of this review, this will be referred to as arthroplasty) has become increasingly popular. The intended long-term benefits of arthroplasty should lead to less recurrent disability, pain, and concomitant surgery. However, it is important to realize that these benefits are yet to be demonstrated $[14,16,17]$. It will probably take several years before the long-term results are known with a reasonable degree of certainty. Meanwhile, to offer patients the best evidence-based treatment, it is important to verify whether the short-term results of arthroplasty are at least similar to those of fusion, and how they evolve over time. If arthroplasty was shown to be less effective than fusion at 1 to 2 years after surgery, a more conservative approach to starting new trials and including new patients in ongoing trials would be justified, while waiting for truly long-term results. The goal of this systematic review is therefore to determine which treatment, discectomy with fusion or discectomy with arthroplasty, provides the best clinical, functional, and radiological outcomes for patients with symptomatic single-level cervical degenerative disc disease. A preliminary search only identified randomized controlled trials (RCTs) that compared fusion and arthroplasty; there were none that compared all of the aforementioned treatment arms combined, or simple discectomy compared with arthroplasty alone. Simple discectomy was therefore not included in this review. In a future update of the review, it might be possible to include simple discectomy as well. 
This review is adopted from the Cochrane Review "Boselie TFM, Willems PC, van Mameren $\mathrm{H}$, de Bie $\mathrm{R}$, Benzel EC, van Santbrink H. Arthroplasty versus fusion in single-level cervical degenerative disc disease. Cochrane Database of Systematic Reviews 2012, Issue 9. Art. No.: CD009173. DOI: 10.1002/14651858.CD009173.pub2." Copyright Cochrane Library, reproduced with permission.

\section{Materials and methods}

We only included RCTs directly comparing fusion with arthroplasty. Inclusion and exclusion criteria are depicted in Table 1.

Clinical, functional, and radiological outcome parameters with a minimal follow-up duration of 1 year were included (Table 2).

Table 1. Inclusion and Exclusion Criteria

\begin{tabular}{ll}
\hline Inclusion Criteria & Exclusion Criteria \\
\hline Randomized controlled trial & Metabolic bone disease (e.g., osteoporosis) \\
Skeletally mature patients (18 yr or older) & Multiple levels \\
Male or female & Previous cervical spine surgery \\
$\begin{array}{l}\text { Symptomatic cervical degenerative disc disease } \\
\text { (radicular pain and/or myelopathy) }\end{array}$ & Malignancy \\
Single level & Radiotherapy of the cervical spine region \\
C3-C4, C4-C5, C5-C6, or C6-C7 & \\
$\begin{array}{l}\text { Insufficient relief after > 6 wk conservative treatment } \\
\text { (except in case of progressive myelopathy) }\end{array}$ & Inflammatory spinal arthritis \\
\hline
\end{tabular}

Table 2. Primary and Secondary Outcomes

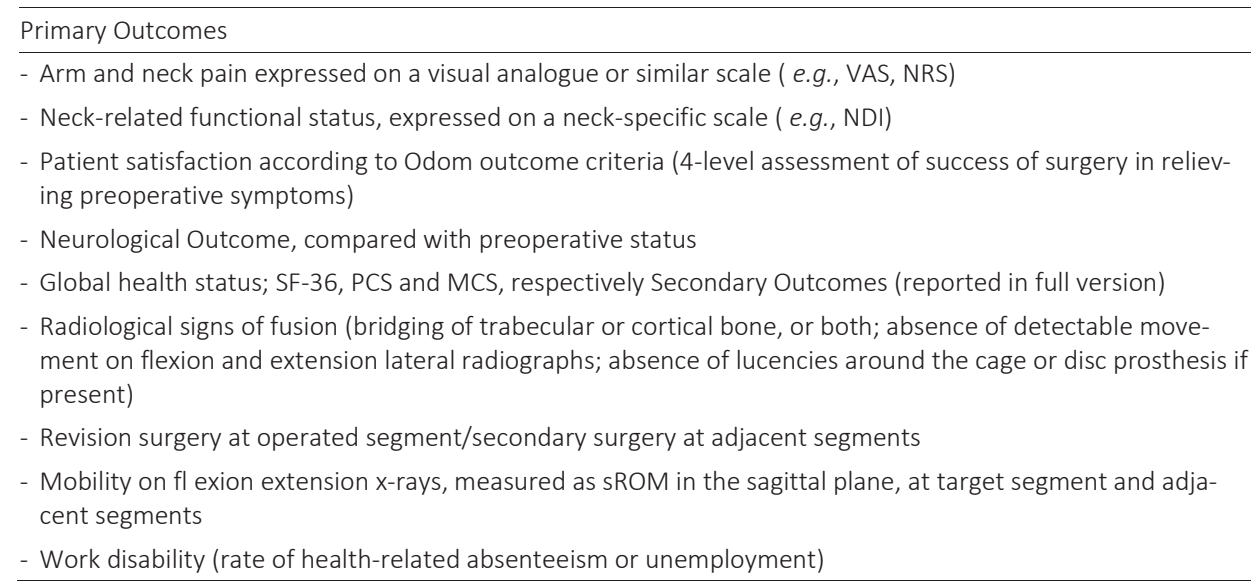

VAS indicates visual analogue scale; NRS, numerical rating scale; NDI, neck disability index; SF-36, Short Form36; PCS, physical component summary; MCS, mental component summary; sROM, segmental range of motion. 
We collected all outcome measures at short-term follow-up (up to and including 3 months) and medium-term follow-up (1-2 year). At the moment of writing the protocol, the authors were not aware of reports of studies with a follow-up longer than 2 years. Some abstracts and articles reporting results on follow-up longer than 2 years were published since then. To avoid introduction of heterogeneity because of large differences in the duration of follow-up, we decided to extract 1-year results when available, if these were not available, we extracted 2-year results. Due to the very limited amount of studies reporting results after more than 2 years' follow-up, these were not included as an additional time point in this review. Results on long-term follow-up (preferably 5 years or longer) will be included in a future update of this review.

We searched the following electronic databases: the CENTRAL [Cochrane Central Register of Controlled Trials] (The Cochrane Library, most recent issue), MEDLINE (through PubMed; from 1950 to May 25, 2011), EMBASE and EBMR (through Ovid; from 1989 to May 25, 2011), SIGLE [System for Information on Grey Literature] database through OpenSIGLE, US FDA [Food and Drug Administration] database on medical devices, Clinicaltrials.gov to identify trials in progress. The exact search strategy can be found in the full version and in Appendix 1 at the end of this chapter, as well as online: http://links.Iww.com/SLA/A408.

\section{Data collection and analysis}

One review author (T.B.) performed a primary screening to remove all references that were clearly not eligible, retrieved the conference proceedings and full-text articles to be evaluated, and contacted study authors of eligible studies for additional information or data if necessary.

\section{Selection of studies}

Three review authors (T.B., P.W., and H.V.S.) independently performed the first selection based on title and abstract. If there was disagreement between review authors, it was resolved through discussion. If consensus could not be reached, a fourth review author (R.D.B.) was consulted. To increase the chance of detecting a publication bias, we have not only included full-text publications, but also abstracts and conference proceedings with sufficient (numerical) data, or if data were made available through personal communication.

This was also the procedure if a study did not meet one of the inclusion or exclusion criteria (e.g., if a study included single-level and multilevel cases, we contacted the authors if data or a publication for the subgroup having undergone single-level surgery were available). 


\section{Data extraction and management}

We used a standardized data extraction form to extract relevant data from selected articles. This was performed independently by 2 review authors for each study. If consensus was not reached between the 2 review authors, a third author was asked to arbitrate. One review author entered the data into RevMan 5.1 (The Nordic Cochrane Center, Copenhagen, Denmark), after which another review author checked all values [18].

Arm pain and neck pain scores were reported as visual analogue scale (VAS) or NRS scores, with a maximum score of 10,20 , or 100 . For the purpose of pooling we recalculated all values to a maximum score of $100 \%$ of the scale.

\section{Assessment of risk of bias in included studies}

We used the 12 criteria recommended in the 2009 updated Cochrane Back Review Group (CBRG) guidelines, which are based on the updated "Cochrane Handbook for Systematic Reviews of Interventions" to assess risk of bias [19, 20]. Two review authors independently applied them to the selected studies (T.B., and H.V.S. or P.W.) and scored the criteria as "low" or "high" risk of bias, or "unclear." In case of disagreement, the 2 review authors discussed until consensus was reached. If consensus was not reached, a third review author (H.V.S. or P.W.) was consulted.

We defined a study as having a "low" overall risk of bias when 6 or more of the 12 criteria were met and there were no serious methodological flaws. We explored the impact of including studies with a high risk of bias by conducting a sensitivity analysis.

Blinding the patient and/or care provider is almost impossible in these types of trials, but not entirely [21]. This is a large potential source of bias, especially in the short term, when a "new-technology" bias can play a role and many of the outcome measures are patient reported. Given that all but one of the primary outcomes assessed in this review are patient-reported outcomes (e.g., pain, satisfaction) the outcome assessor was regarded as not blinded if the patient was not blinded.

\section{Measures of treatment effect}

\section{Dichotomous data}

Dichotomous data were used for reporting on: fusion (fused segment or not), neurological status (unchanged or improved, vs. worsened), working status (working or not working), and patient satisfaction ((very) satisfied vs. all other options). These were reported as risk ratios (RRs) with 95\% confidence intervals (Cls). 


\section{Continuous data}

For continuous data (e.g., VAS, neck disability index, sROM), we used mean differences (MDs) if outcomes were measured in the same way between trials. We calculated 95\% Cls for each outcome.

\section{Clinical relevance of results}

We used the 5 questions recommended by the CBRG to determine whether the results of the individual studies were clinically relevant [19]. This effect was compared with the preoperative status. To determine clinical relevance of the results of the meta-analysis we used the pooled effect sizes. The difference in effect size between the 2 treatments for each outcome at each time point was scored as "small," "medium," or "large" [20]. These were defined as follows:

- Small: MD less than $10 \%$ of the scale, RR more than 0.8 (or $<1.25$, depending on the risk/benefit being for the investigational or the control group);

- Medium: MD 10\% to $20 \%$ of the scale, RR from 1.25 to 2.0 or from 0.5 to 0.8 ;

- Large: MD more than $20 \%$ of the scale, RR less than 0.5 or more than 2.0.

\section{Dealing with missing data}

If possible, we contacted the original investigators to request any missing information. We explored the impact of including studies with high levels of missing data (15\% or greater loss to follow-up) in the overall assessment of treatment effect by using a sensitivity analysis.

If standard deviations (SDs) were not reported and could not be acquired, one of 3 options was used; the first option was calculation of the missing SDs if sufficient other data were reported (MDs, $P$ value, number of observations), the second was actual manual measurement (if graphs with error bars were present), and the third option was imputation by taking the average SD of similarly sized studies for that outcome.

\section{Assessment of heterogeneity}

We assessed the presence of heterogeneity between the trials primarily on a visual inspection of the overlap of $\mathrm{Cls}$ in the forest plots (eye-balling), followed, if necessary, by a $Q$ test ( $\chi 2$ analysis) to test for heterogeneity and an $l^{2}$ analysis to describe the proportion of total variance that can be explained by heterogeneity.

\section{Assessment of reporting biases}

We planned the use of a funnel plot to identify possible publication bias. We placed no language restrictions in the search terms to reduce language, and possibly location bias. 
We actively searched for publications using the same group of subjects (duplicate populations) to ensure we did not inadvertently count them as separate studies.

\section{Data synthesis}

We assessed the overall quality of the evidence for each outcome. To accomplish this, we used an adapted GRADE approach, as recommended by the updated CBRG method guidelines [19]. The quality of the evidence on a specific outcome was based on the performance against 5 domains: study design (methodological quality), consistency of results, directness (generalizability), precision (sufficient data), and reporting of the results across all studies that measure that particular outcome (possibility of publication bias).

The quality starts at "high" when RCTs with a low risk of bias provide results for the outcome, and reduces by a level for each of the factors not met.

Meta-analyses were only conducted where studies were judged to be sufficiently homogeneous with respect to the included population, treatments, and outcome measure, as we expected those to be the most important reasons for clinical heterogeneity. Because our inclusion criteria were quite strict (e.g., no prior surgery, only single-level cases, only adults) we expected these in fact to be fairly homogeneous. Unless stated otherwise, a fixed-effect model was used for the pooled analysis. In case of significant heterogeneity a random-effects model was used, and an effort was made to identify possible causes. Significant heterogeneity was defined as $P$ less than 0.1 in the $\chi 2$ analysis, combined with an $l^{2}$ more than $30 \%$.

\section{Sensitivity analysis}

Two sensitivity analyses were planned a priori; one to assess the influence of including studies with a high risk of bias, and one to the influence of high levels of attrition ( $>15 \%$ loss to follow-up). Six additional post hoc sensitivity analyses were performed. These are described in detail in the full-text version.

\section{Results}

The search was performed on May 25, 2011, and 5847 references were identified in the primary search (see flow chart in Figure 1), and 49 through other sources (e.g., Clinicaltrials.gov). 


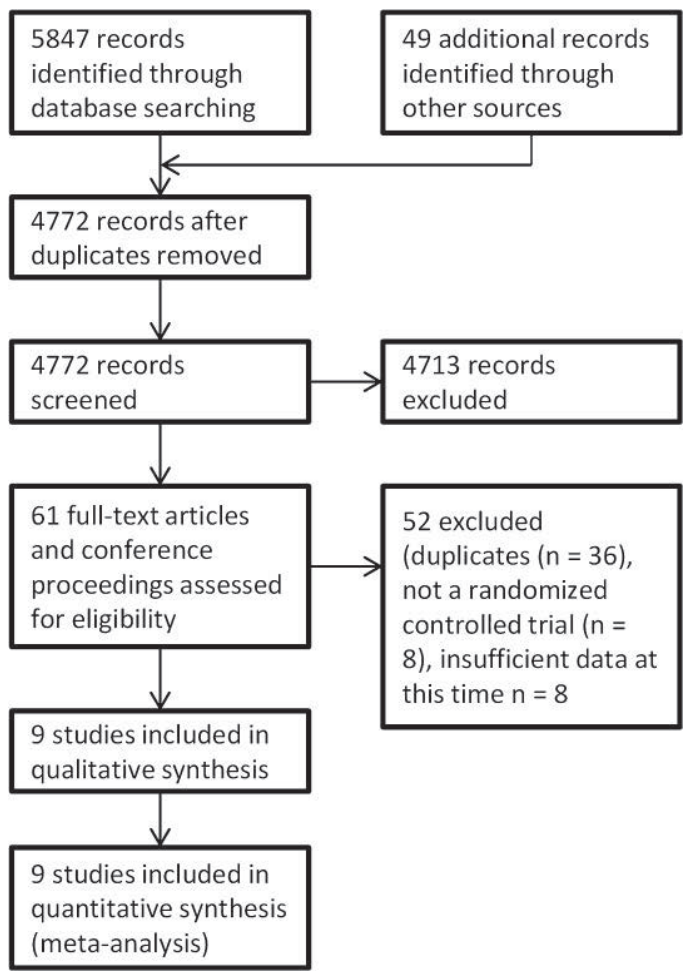

Figure 1: Flow chart of the search showing number of inclusions/exclusions at each step of the review process.

After removal of 1124 duplicate references, the total amount of references to be screened was 4773 . After a primary screening by one author (T.B.) a total of 57 publications seemed eligible for inclusion, other references were not selected for various reasons (e.g., publications about the lumbar spine, retrospective studies, studies without a control group). The high amount of exclusions during primary screening was caused by the relatively wide search terms that were used to keep the amount of "missed references" to a minimum. Four additional publications were identified through cross-referencing; however, all 4 were duplicate populations. These 61 publications reported on a total of 24 separate study populations. Of these 24 study populations, 9 were included [22-74], 7 were excluded [75-81], one is awaiting classification [82], and 7 were ongoing $[21,22,25,27,28,83-90]$. Additionally, one of the 61 references did not report about a study population but described a surgical technique and was therefore excluded [91]. If there were multiple publications reporting about a study, they were grouped and we extracted data for the publication with the largest sample size for that outcome. Studies were classified as ongoing if results were not available at the time of writing of this review, or if the results that had been reported included (partial) duplicate populations of an already included study. This was the case for 3 studies (the FDA IDE [Investigational Device Exemption] studies concerning the Advent disc (Orthofix, Lewisville, TX, USA), 
Mobi-C disc (LDR Medical, Austin, TX, USA), and Discover disc (DePuy Spine, Raynham, MA, USA)). Several studies were ongoing with the purpose of acquiring long-term followup (up to 10 years) at the time of classification, but had reported results for a follow-up of at least 1 year; these were solely classified as included and not also as ongoing.

In compliance with the new MECIR (Methodological Expectations for Cochrane Intervention Reviews) the search was updated July 26, 2012. This resulted in a total of 523 additional references. Of these references, 24 seemed eligible during the primary screening. Thirteen of these were references reporting data about studies that were already included; the other 11 references were classified as "Studies awaiting classification."

\section{Included studies}

The 9 included study populations included a total of 2400 participants (1262 investigational, 1138 control). Study sample size was in the range of 55 to 541 . All studies were RCTs directly comparing arthroplasty with fusion. The majority of the studies (7 of 9) were conducted in North America, all of which were multicenter US FDA IDE studies. One study was conducted in Germany [65] and one international study was conducted in the UK, Switzerland, Australia, and Belgium [73].

Eight of the studies were industry sponsored [23, 34, 49, 56, 58, 62, 70, 73].

\section{Excluded studies}

Seven studies were excluded. One because it was retrospective [80], 5 because they were not an RCT [75-78, 81], and one because it combined a prospective cohort with one of the treatment arms of an RCT [92].

\section{Risk of bias in included studies}

A summary of the "risk of bias" analysis for each of the included studies is depicted in Table 3. Five studies had a low overall risk of bias $[34,49,56,58,62]$, and 4 studies had a high overall risk of bias $[23,65,70,73]$. In all 4 studies with a high overall risk of bias, this was because of a large number of quality criteria being judged as having an "unclear risk of bias." We identified no studies with a fatal flaw. The risk of bias analysis is described in more detail in Appendix 2 at the end of this chapter, as well as online: http://links.Iww.com/SLA/A409. 
Table 3. Risk of Bias Analysis

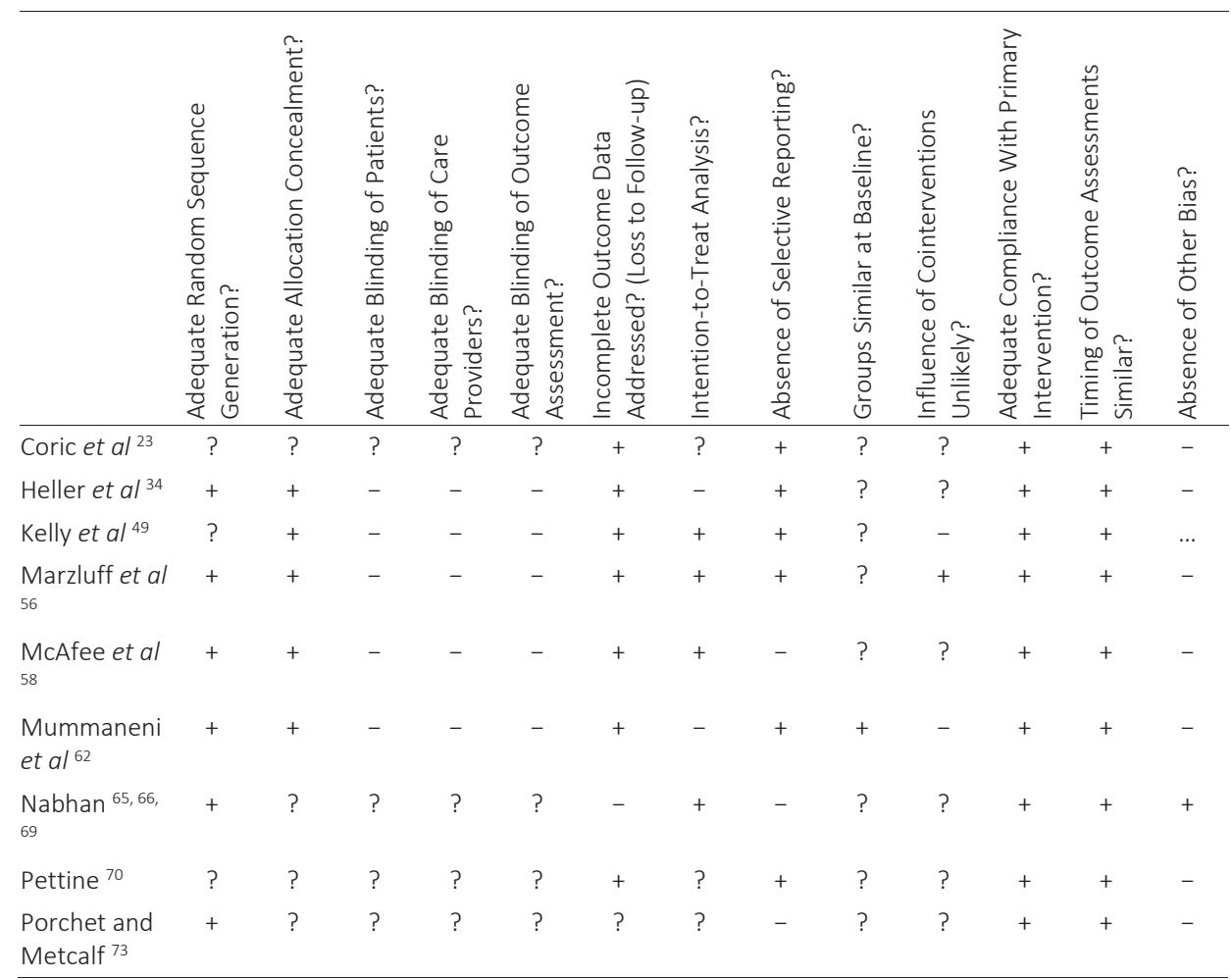

The criteria are scored with a " + " if there is suffi cient data to support that they are met in the study; a "?" if there is insuffi cient data to support this; and a "-" if there is data that points to the criteria not being met.

\section{Primary outcomes}

\section{Arm pain}

For arm pain, 6 studies were identified with a total of 1346 (at 3 months) and 1310 (at 12 years) participants $[34,56,62,65,70,73]$. All 6 of these were suitable for pooled analysis, 3 of which had an overall low risk of bias [34, 56, 62]. There was low quality evidence (risk of bias, reporting) that there was a significant difference between arthroplasty and fusion at 3 months and 1 to 2 years, in favor of arthroplasty $(\mathrm{MD},-2.18 ; 95 \% \mathrm{Cl},-3.68$ to -0.68 ; $\mathrm{MD},-1.54 ; 95 \% \mathrm{Cl}-2.86$ to -0.22 , respectively) (Figure 2 ). 


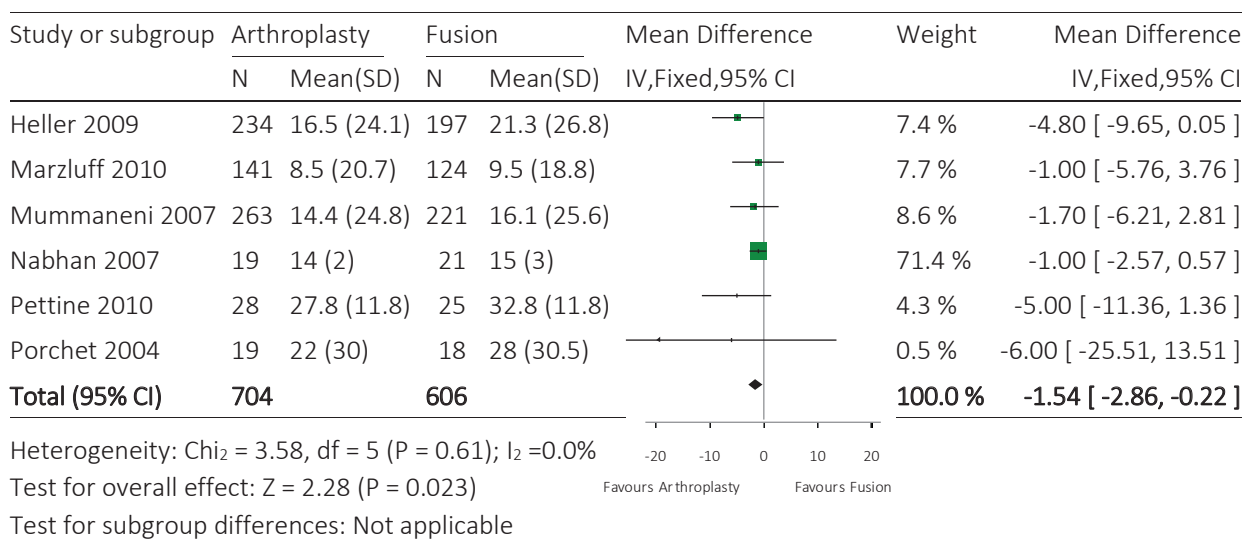

Figure 2: Forest plot of VAS scores for arm pain at 1 to 2 years after surgery showing MD between the 2 treatment arms. VAS indicates visual analogue scale; MD, mean difference; $\mathrm{Cl}$, confidence interval; SD, standard deviation.

Clinical relevance was low because the pooled difference in effect size was small $(<10 \%$ of the scale). For 1 study the data for VAS pain could not be used for pooled analysis of either arm pain or neck pain [23]. They used a combined VAS score, which was defined as overall pain in arms, shoulders, and neck. They reported a small difference in favor of arthroplasty at 3 months and 1 to 2 years, both of which were not significant $(P=0.58$ and $P=0.07$, respectively).

Size and direction of the effect corresponded to the pooled analysis of VAS arm and VAS neck.

\section{Neck pain}

For neck pain, 6 studies were identified with a total of 1347 (at 3 months) and 1309 (at 1-2 years) participants $[34,56,62,65,70,73]$. All 6 of these were suitable for pooled analysis, 3 of which had an overall low risk of bias $[34,56,62]$. There was moderate quality evidence (risk of bias) that there was no significant difference between arthroplasty and fusion at 3 months (MD, $-3.67 ; 95 \% \mathrm{Cl},-9.80$ to 2.46). For this analysis, a random effects model was used due to a large amount of heterogeneity ( $\chi 2=36.32$ at degrees of freedom $\left.=5, I^{2}=86 \%\right)$. This was found to be caused by the extremely small SDs of one study [65].

There was moderate quality evidence (risk of bias) that there was a significant difference between arthroplasty and fusion at 1 to 2 years, in favor of arthroplasty (MD, - 3.12; $95 \% \mathrm{Cl},-4.69$ to -1.28$)$. Clinical relevance was low because the pooled difference in effect size was small $(<10 \%$ of the scale).

Neck-related functional status

For neck-related functional status, 6 studies were included with a total of 1545 (at 3 months) and 1505 (at $1-2$ years) participants [23, 34, 56, 62, 70, 73]. All 6 of these were 
suitable for pooled analysis, 3 of which had an overall low risk of bias $[34,56,58]$. There was moderate quality evidence (risk of bias) that there was a significant difference between arthroplasty and fusion at 3 months and 1 to 2 years, in favor of arthroplasty (MD, - 5.14; $95 \% \mathrm{Cl},-6.94$ to $-3.34 ; \mathrm{MD}-2.79 ; 95 \% \mathrm{Cl},-4.73$ to -0.85 , respectively) (Figure $3)$. Clinical relevance was low because the pooled effect size was small $(<10 \%$ of the scale).

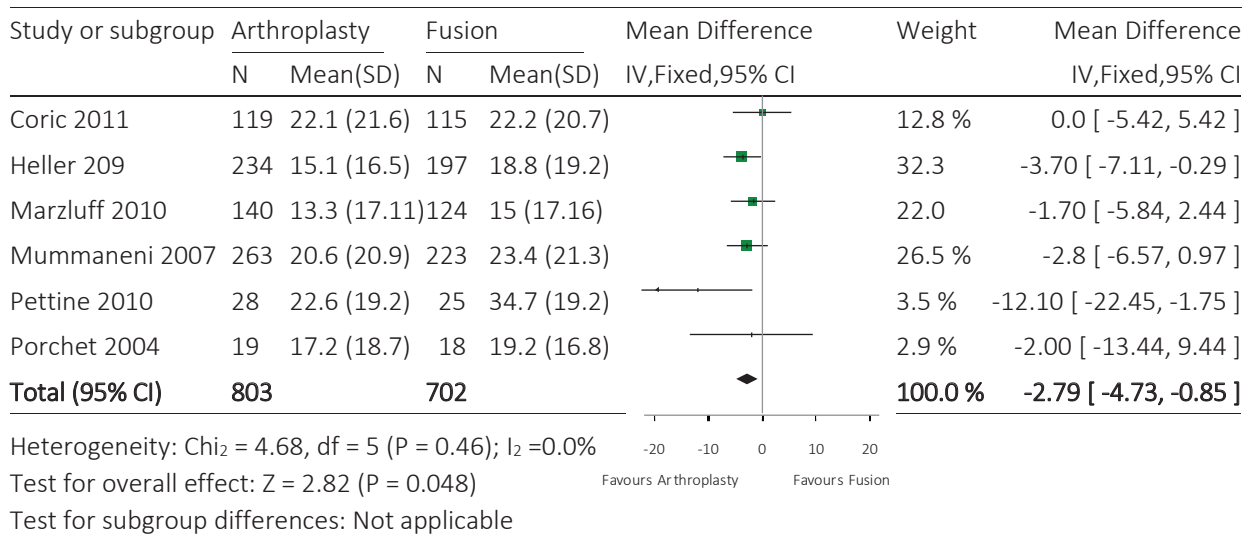

Figure 3: Forest plot of NDI scores at 1 to 2 years after surgery showing MD between the 2 treatment arms. NDI indicates neck disability index; $\mathrm{MD}$, mean difference; $\mathrm{Cl}$, confidence interval; SD, standard deviation.

\section{Patient satisfaction}

For patient satisfaction, 2 studies were included with a total of 498 participants $[23,56]$. Both studies were suitable for pooled analysis, one with an overall low risk of bias [56]. Only results at 1 to 2 years were reported. There was very low quality evidence (risk of bias, consistency, precision) that there was no significant difference between arthroplasty and fusion at 1 to 2 years (RR, 1.06; 95\% Cl, 1.00-1.12; $P=0.06)$.

\section{Neurological status}

For neurological status, defined as the percentage of patients with unchanged or improved neurological status, at 3 months only 1 study, with a total of 497 participants, was included [62].

This study had an overall low risk of bias. There was low quality evidence (risk of bias, precision) that there was no significant difference between the 2 treatment groups (RR, 1.05; 95\% Cl, 0.99-1.12; $P=0.09$ ).

Three studies, with a total of 1147 participants, were included for the results at 1 to 2 years [23, 34, 62]. All 3 of these were suitable for pooled analysis. Two of these had an overall low risk of bias [34,62]. There was moderate quality evidence (risk of bias) that there was a significant difference between the 2 treatment groups in favor of arthroplasty (RR, 1.05; 95\% Cl, 1.01-1.09; $P=0.007$ ). 


\section{Global health status}

For the Short Form-36 physical component summary (SF-36-PCS) at 3 months, only 1 study was included. 34 There was low quality evidence (risk of bias, precision) that there was a significant difference between the 2 treatment groups in favor of arthroplasty (MD, 2.40; 95\% Cl, 0.55-4.25). Clinical relevance was low because the pooled effect size was small $(<10 \%$ of the scale).

For the SF-36-PCS at 1 to 2 years, 3 studies were included [34, 62, 73]. All 3 were suitable for pooled analysis. Two of the studies had an overall low risk of bias [34, 62]. There was moderate quality evidence (risk of bias) that there was a significant difference between the 2 treatment groups in favour of arthroplasty (MD, 2.10; 95\% Cl, 0.68-3.51). Clinical relevance was low because the pooled effect size was small ( $<10 \%$ of the scale).

For the SF-36- mental component summary (MCS) at 3 months only 1 study was included. 34 There was very low quality evidence (risk of bias, precision [very serious]) that there was no significant difference between the 2 treatment groups (MD, 1.80; 95\% Cl, 0.10 to 3.70). Clinical relevance was low because the pooled effect size was small $(<10 \%$ of the scale).

For the SF-36-MCS at 1 to 2 years, 3 studies were included [34, 62]. All 3 were suitable for pooled analysis. Two of the studies had an overall low risk of bias [34,62]. There was low quality evidence (risk of bias, consistency) that there was a significant difference between the 2 treatment groups in favour of arthroplasty (MD, 1.46; 95\% Cl, 0.10-2.82). Clinical relevance was low because the pooled effect size was small ( $<10 \%$ of the scale).

One study was not included in the pooled analysis because it reported SF-36 scores as the percentage change from baseline at 1 to 2 years [56]. Results for this study showed $91 \%$ of the arthroplasty patients to have an unchanged or improved SF-36-PCS score versus $92 \%$ in the fusion group. For the SF-36-MCS this was $77 \%$ versus $71 \%$, respectively. Similar to the pooled analysis, there were no large differences between the groups. Direction of effect for the SF-36-PCS at 1 to 2 years seems to be the inverse of the pooled results, but this cannot be compared directly due with absence of actual scores.

\section{Sensitivity analysis}

For the 2 most important outcomes, arm pain and neck-related functional status, the results of the sensitivity analyses were pooled (Figures 4, 5). These figures illustrate that size and direction of the effect were consistent in these analyses. However, for arm pain a small study effect could not be ruled out. In this analysis, statistical significance was lost, although only a small proportion of participants was excluded (up to 11\%). Thus, it is unlikely that this null finding is simply due to a decrease in power (i.e., a type II error). 


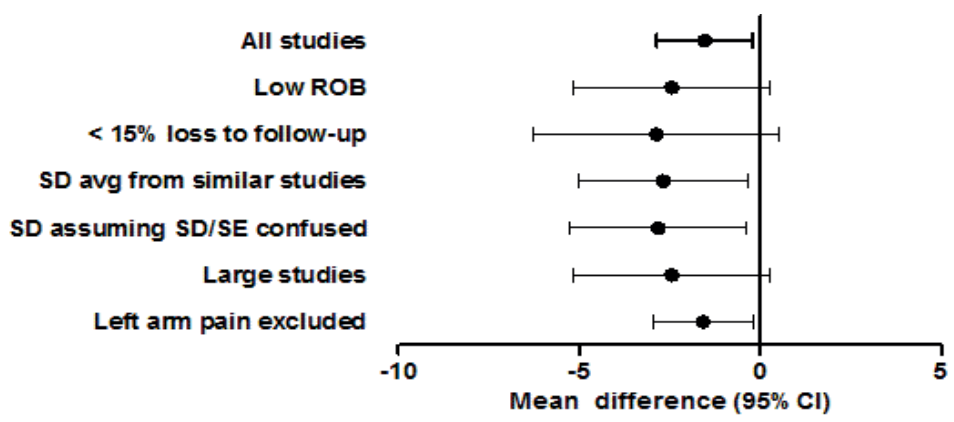

Figure 4: Sensitivity analysis of VAS scores for arm pain at 1 to 2 years after surgery showing a consistent size and direction of difference in effect size. VAS indicates visual analogue scale; SD, standard deviation; $\mathrm{Cl}$, confidence interval.

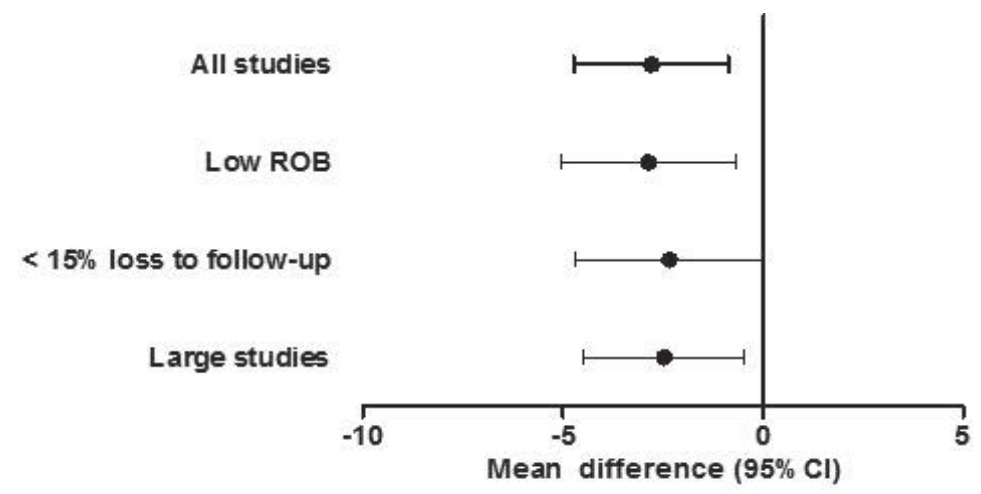

Figure 5: Sensitivity analysis of NDI scores at 1 to 2 years after surgery showing a consistent size and direction of difference in effect size. NDI indicates neck disability index; $\mathrm{Cl}$, confidence interval.

For neck-related functional status this was not the case and findings were robust to sensitivity analyses.

Although no significant difference in neck pain scores was found at 3 months in the main analysis, a significant difference was found in all sensitivity analyses. No significant difference was found in one study that had extremely low SDs compared with all the other, often much larger, studies [65].

The substantial heterogeneity that was present was not evident when these SDs were replaced with the average of the other small studies, and when they were recalculated under the assumption that for this study inadvertently SEs instead of SDs were reported. Results at 1 to 2 years were robust to sensitivity analyses.

Patient satisfaction gained significance in the analysis excluding studies with a high risk of bias, but this was based only on one study. The comparison for neurological status at 1 to 2 years lost significance in the analysis excluding studies with more than $15 \%$ loss to follow-up. This could be a type II error because size and direction of the effect were similar, whereas more than $40 \%$ of the participants was excluded. This was also the case 
for results at 1 to 2 years of the SF-36-MCS, for which significance was lost, but a similar direction and size of effect were seen. The SF-36-PCS was robust to sensitivity analyses. Due to a paucity of studies, no sensitivity analyses could be performed for working status at either time point.

\section{DISCUSSION}

\section{Summary of main results}

A summary of results for the 7 main outcomes is reported in the "Summary of Findings" table (see Appendix 3 at the end of this chapter, or online: http://links.Iww.com/SLA/A410). There was low quality evidence that arm pain was significantly lower in the arthroplasty group at 3 months and 1 to 2 years. However, the actual difference was small and not clinically relevant (2.18 points lower on a 100-point scale for results at 3 months and 1.54 points lower on a 100-point scale for results at 1-2 years). Significance was not robust to sensitivity analyses, which was unlikely to be solely the result of a type II error. However, size and direction were comparable in all sensitivity analyses (Figures 4, 5).

There was moderate quality evidence that neck pain was significantly lower and neckrelated functional status (neck disability index) was significantly higher in the arthroplasty group at 3 months and 1 to 2 years. But again, the actual differences between the groups were small (2.79-5.14 points on a 100-point scale), and not clinically relevant. This was also true for the SF-36-PCS. The SF-36-MCS also showed a small, significant difference in favor of arthroplasty, but significance was not robust to sensitivity analyses.

With respect to the secondary outcomes there were 2 statistically significant and clinically relevant outcomes: moderate quality evidence that for arthroplasty there was a large reduction in revision surgery at the index level as compared with fusion, and high quality evidence of a large difference in SROM at the index level at 3 months and 1 to 2 years, being caused by a reduction of SROM in the fusion group, whereas average SROM in the arthroplasty group was equal to baseline. Whether this correlates with better clinical or functional outcome has not been investigated. The reduction in revision surgery was not robust to sensitivity analyses.

No statistically significant reduction in adjacent level surgery was seen, although there was a trend in favor of arthroplasty. Until a statistically significant, and clinically relevant, difference is confirmed in longer-term follow-up, this should not lead to any conclusions.

In summary, a clinically relevant difference was not seen for any of the primary outcomes, although results for the arthroplasty group were better than the fusion group for all comparisons, and were often statistically significant. 


\section{Overall completeness and applicability of evidence}

Due to the strict inclusion criteria we used, the generated evidence cannot be applied to all patients experiencing single-level cervical degenerative disc disease. The vast majority of the study population did not have any prior cervical spine surgery, which is not a reflection of daily practice, but results in more homogeneous groups and a sound comparison. With respect to the other patient characteristics, generalizability was much better because these indications and contraindications were much more of a reflection of daily practice. Therefore, the conclusions of this review are primarily relevant for patients with a first presentation of radiculopathy or myelopathy due to single-level cervical degenerative disc disease that is refractory to nonsurgical treatment.

\section{Quality of the evidence}

Overall, quality of the evidence was very low to moderate. This was, at least partially, due to the downgrading of outcomes that were reported by patient or carer in a nonblinded setting. Until blinded studies on this subject become available a "new technology" bias cannot be ruled out. We expect this type of bias to fade over time. In our opinion it was debatable whether this would already be so after 1 or 2 years. This is why this conservative approach was chosen. We do think that "new technology" bias will diminish in "longer-term" (4 to 5 years or longer) results.

For several studies, many criteria had to be judged as "unsure" in the risk of bias analysis. In many cases a detailed protocol could not be found, and additional information could not be acquired through study authors or sponsors. This led to several studies being judged to have an overall high risk of bias. We suspect that at least some of these criteria were in fact eligible for a low risk of bias score. This conservative scoring of the studies may affect the quality of evidence scores in a negative way. We therefore strongly advise authors and sponsors to publish study protocols of RCTs to allow for a more reliable scoring of the current level of evidence.

\section{Potential biases in the review process}

We only included studies written in English, although we considered trials in all languages to minimize publication bias. To minimize chances of publication bias further, we did an extensive database search and decided to include unpublished studies that surfaced (i.e., no full-text report of the full population), contrary to what we planned in the protocol. It is difficult to say if inclusion of unpublished studies leads to overestimation or underestimation of the effect $[20,93]$. In this review, we included 2 studies based on conference proceedings [56, 70], for 1 of these, we received all requested data for the entire population from the sponsor [56], which eliminates the possibility of a subset showing a 
more/less favorable effect. This effect cannot be ruled out for the other study [70], although direction and size of the effect were generally in line with the other included studies.

Another potential source of bias is the fact that we only included studies in which participants did not have prior surgery at the level of surgery, or on the superior or inferior adjacent level. This led to the partial exclusion of studies $[49,58]$. However, we are of the opinion that previous surgery can be a serious confounding factor in this type of surgery.

It could be argued that the large variety in type of disc prosthesis that was evaluated attenuates applicability of the conclusion of this review to specific prostheses. Although the design of the various types of prostheses varies, they all have the same basic principle. One type may have results that differ from others, but large differences should be identified through the analysis of heterogeneity. If more studies become available for each type of prosthesis, a subgroup analysis could be useful. However, given the paucity of studies per device, this is currently not feasible.

Finally, a potential source of bias was related to the manner by which we handled some of the missing data, by several methods of imputation. Our use of these methods was found to have no effect on significance, effect size, or direction of effect in a sensitivity analysis.

\section{Agreements and disagreements with other studies or reviews}

Since the publication of our protocol, 5 reviews have been published [94-98]. Overall, not all of our findings were consistent with the other reviews. In our review, neurological status and reoperation rate at the index level were consistently significantly in favor of arthroplasty. Although other reviews showed an effect in the same direction, these comparisons did not consistently reach statistical significance. A likely cause is the fact that these reviews invariably included fewer studies and participants than the current review. Given the small differences between the 2 groups for all outcomes, the number of included participants is a highly relevant factor. This is supported by the fact that there were no cases in which the direction of effect was not the same.

\section{Conclusion}

\section{Implications for practice}

Although differences in effect size were statistically significant, effects were invariably small and not clinically relevant for all primary outcomes. Significance was often gained or lost in the varying sensitivity analyses. Nevertheless, the direction of the effect was consistently in favor of arthroplasty. Given that all of the included studies were not 
blinded this could be because of patient or carer expectations. At this time, neither treatment option is the obvious first choice, with both treatments being valid treatment options with respect to results at a maximum of 2 years. Given the current absence of truly long-term results to provide evidence of the intended advantage of arthroplasty over fusion, that is a reduction in ASDis, and absence of long-term complications (e.g., fusion around the prosthesis, durability issues), use of these devices should still be limited to clinical trials.

\section{Implications for Research}

Minimal differences in short-term results is to be expected because the primary aim of the operation, decompression of the nerve root, should be similar in both treatment groups. This is supported by the fact that, although there is a tendency for results to be in favor of arthroplasty, the pooled differences in effect size were in general small. Given the fact that all included studies were not blinded, this could be the result of patient or carer bias. The intended goal of disc prosthesis implantation is the prevention of new symptoms in the long term. It is therefore imperative that long-term results are reported for all of the trials that have been performed to date, and for those that are ongoing because only long-term results can indicate the effectiveness of these devices in preventing long-term sequelae, such as ASDis or adjacent disc degeneration. Contrary to this systematic review, which featured primarily patient-reported primary outcomes, a future update should therefore include these long-term sequelae as one of the primary outcome measures.

It will be interesting to see if patient-reported outcomes will continue to be in favor of arthroplasty, especially because we consider it is unlikely that patient expectations will continue to play a role after several years. Based on our review and experiences, there are several suggestions we would like to make for future reports on these RCTs in order to facilitate future meta-analyses, and ensure the highest quality of evidence. These recommendations are as follows:

1. More detailed reporting of study methodology, ideally by publishing study protocols;

2. Reporting numerical values with their variance and the number of patients evaluated, for all time points;

3. Reporting actual values at the time points, as well as the changes in scores and their variance;

4. More detailed reporting of baseline demographics;

5. Reporting results separately for subgroups (single- vs. multiple-level, prior cervical spine surgery vs. no previous cervical spine surgery). 


\section{Key Points}

- Nine study populations, with a total of 2400 included participants, were included in this systematic review. For the primary outcome, arm pain, 6 studies were identified with a total of 1346 (at 3 months) and 1310 (at 1-2 years) participants.

- Risk of bias analysis showed a low overall risk of bias for 5 of the 9 included studies. In all 4 studies with a high overall risk of bias, this was caused by a large number of quality criteria being judged as having an "unclear risk of bias."

- A clinically relevant difference was not seen for any of the primary outcomes, although results for the arthroplasty group were better than the fusion group for all comparisons, often statistically significant.

- At this time, neither treatment option is the obvious first choice, with both treatments being valid treatment options with respect to results at a maximum of 2 years. Given the absence of truly long-term results, use of these devices should still be limited to clinical trials.

\section{Acknowledgments}

The authors thank Rachel Couban, Trials Search Coordinator of the CBRG, for her help in reviewing the search strategy, adapting it for the various search engines, and performing the initial search. The authors also thank Victoria Pennick, former Managing Editor of the CBRG, for her guidance in the preparation of the protocol, and Teresa Marin, Managing Editor of the CBRG, for her guidance in the preparation of the review. Furthermore, the authors also thank Qionghui Joanna Liu, B.Eng, of the Institute for Work \& Health, for her help in extracting data from a publication in Chinese. $81 \mathrm{In}$ addition, the authors thank $\mathrm{D}$. Coric, MD; J. Heller, MD; J. Hipp, PhD; J. Marzluff, MD; A. Nabhan, MD, PD; K. Pettine, MD; F. Philips, MD; and F. Porchet, MD, PD, for providing additional data that were not reported in their publications. Finally, the authors thank K. Baker, PhD, of Globus Medical, and D. Wootten, PhD, of Medtronic Sofamor Danek, for sharing the original data for the 4 concerned trials. Supplemental digital content is available for this article. Direct URL citations appearing in the printed text are provided in the HTML and PDF version of this article on the journal's web site (www.spinejournal.com). 


\section{References}

1. Gore, D.R. and S.B. Sepic, Anterior cervical fusion for degenerated or protruded discs. A review of one hundred forty-six patients. Spine (Phila Pa 1976), 1984. 9(7): p. 667-71.

2. Gore, D.R. and S.B. Sepic, Anterior discectomy and fusion for painful cervical disc disease. A report of 50 patients with an average follow-up of 21 years. Spine (Phila Pa 1976), 1998. 23(19): p. 2047-51.

3. Klein, G.R., A.R. Vaccaro, and T.J. Albert, Health outcome assessment before and after anterior cervical discectomy and fusion for radiculopathy: a prospective analysis. Spine (Phila Pa 1976), 2000. 25(7): p. 8013.

4. White, A.A., 3rd, et al., Relief of pain by anterior cervical-spine fusion for spondylosis. A report of sixty-five patients. J Bone Joint Surg Am, 1973. 55(3): p. 525-34.

5. Wieser, E.S. and J.C. Wang, Surgery for neck pain. Neurosurgery, 2007. 60(1 Supp1 1): p. S51-6.

6. Henderson, C.M., et al., Posterior-lateral foraminotomy as an exclusive operative technique for cervical radiculopathy: a review of 846 consecutively operated cases. Neurosurgery, 1983. 13(5): p. 504-12.

7. Dowd, G.C. and F.P. Wirth, Anterior cervical discectomy: is fusion necessary? J Neurosurg, 1999. 90(1 Suppl): p. 8-12

8. Wirth, F.P., et al., Cervical discectomy. A prospective analysis of three operative techniques. Surg Neurol, 2000. 53(4): p. 340-6; discussion 346-8.

9. Nandoe Tewarie, R.D., R.H. Bartels, and W.C. Peul, Long-term outcome after anterior cervical discectomy without fusion. Eur Spine J, 2007. 16(9): p. 1411-6.

10. Fountas, K.N., et al., Anterior cervical discectomy and fusion associated complications. Spine (Phila Pa 1976), 2007. 32(21): p. 2310-7.

11. Grob, D., Surgery in the degenerative cervical spine. Spine (Phila Pa 1976), 1998. 23(24): p. 2674-83.

12. Persson, L.C., et al., Cervical radiculopathy: pain, muscle weakness and sensory loss in patients with cervical radiculopathy treated with surgery, physiotherapy or cervical collar. A prospective, controlled study. Eur Spine J, 1997. 6(4): p. 256-66.

13. Eck, J.C., et al., Biomechanical study on the effect of cervical spine fusion on adjacent-level intradiscal pressure and segmental motion. Spine (Phila Pa 1976), 2002. 27(22): p. 2431-4.

14. Seo, M. and D. Choi, Adjacent segment disease after fusion for cervical spondylosis; myth or reality? $\mathrm{Br} \mathrm{J}$ Neurosurg, 2008. 22(2): p. 195-9.

15. Hilibrand, A.S., et al., Radiculopathy and myelopathy at segments adjacent to the site of a previous anterior cervical arthrodesis. J Bone Joint Surg Am, 1999. 81(4): p. 519-28.

16. Bartels, R.H., R. Donk, and A.L. Verbeek, No justification for cervical disk prostheses in clinical practice: a meta-analysis of randomized controlled trials. Neurosurgery, 2010. 66(6): p. 1153-60; discussion 1160.

17. Botelho, R.V., et al., A systematic review of randomized trials on the effect of cervical disc arthroplasty on reducing adjacent-level degeneration. Neurosurg Focus, 2010. 28(6): p. E5.

18. Review Manager (RevMan). Version 5.1. 2011, The Nordic Cochrane Centre, The Cochrane Collaboration: Copenhagen.

19. Furlan, A.D., et al., 2009 updated method guidelines for systematic reviews in the Cochrane Back Review Group. Spine (Phila Pa 1976), 2009. 34(18): p. 1929-41.

20. Higgins, J. and S. Green, eds. Cochrane Handbook for Systematic Reviews of Interventions Version 5.1.0 [updated March 2011]. 2011, The Cochrane Collaboration.

21. Arts, M.P., et al., The NEtherlands Cervical Kinematics (NECK) trial. Cost-effectiveness of anterior cervical discectomy with or without interbody fusion and arthroplasty in the treatment of cervical disc herniation; a double-blind randomised multicenter study. BMC Musculoskelet Disord, 2010. 11: p. 122.

22. Coric, D., et al., Prospective study of cervical arthroplasty in 98 patients involved in 1 of 3 separate investigational device exemption studies from a single investigational site with a minimum 2-year follow-up. Clinical article. J Neurosurg Spine, 2010. 13(6): p. 715-21. 
23. Coric, D., et al., Prospective, randomized, multicenter study of cervical arthroplasty: 269 patients from the Kineflex $\mid C$ artificial disc investigational device exemption study with a minimum 2-year follow-up. J Neurosurg Spine, 2011.

24. Guyer, R.D., et al., Prospective, Randomized, Multicenter Trial Comparing Cervical Total Disc Replacement to Anterior Cervical Fusion in 269 Patients: 24-month Follow-up. The Spine Journal, 2010. 10(9): p. S41S42.

25. Guyer, R.D., et al., A Prospective Randomized Comparison of Cervical Disc Replacement and Anterior Cervical Fusion: Combined Results from Five FDA IDE Trials from a Single Site. The Spine Journal. 10(9, Supplement 1): p. S43-S43.

26. Musante, D.B., et al., Comparison of Radiographic Findings of Total Disc Replacement vs. Anterior Cervical Fusion: 24-Month Follow-up from a Prospective, Randomized, Controlled, Multicenter Trial with 269 Patients. The Spine Journal, 2010. 10(9): p. S32-S33.

27. Jawahar, A., et al., Total disc arthroplasty does not affect the incidence of adjacent segment degeneration in cervical spine: results of 93 patients in three prospective randomized clinical trials. Spine J, 2010. 10(12): p. 1043-8.

28. Jawahar, A., et al., Total disc arthroplasty does not affect the incidence of adjacent segment degeneration in cervical spine: Results of 93 patients in three prospective, randomized, clinical trials, in 25th Annual Meeting of the North American Spine Society (NASS). 2010: Miami, FL United States. p. pp 34S

29. Coric, D., F. Finger, and P. Boltes, Prospective randomized controlled study of the Bryan Cervical Disc: early clinical results from a single investigational site. J Neurosurg Spine, 2006. 4(1): p. 31-5.

30. Garrido, B.J., et al., Adjacent-level cervical ossification after Bryan cervical disc arthroplasty compared with anterior cervical discectomy and fusion. J Bone Joint Surg Am, 2011. 93(13): p. 1185-9.

31. Garrido, B.J., T.A. Taha, and R.C. Sasso, Clinical Outcomes of Bryan Cervical Disc Arthroplasty A Prospective, Randomized, Controlled, Single Site Trial With 48-Month Follow-up. J Spinal Disord Tech, 2010.

32. Hacker, R.J., Cervical disc arthroplasty: a controlled randomized prospective study with intermediate follow-up results. Invited submission from the joint section meeting on disorders of the spine and peripheral nerves, March 2005. J Neurosurg Spine, 2005. 3(6): p. 424-8.

33. Heller, J., et al., Bryan Cervical Disc Replacement: Results of the United States Prospective Randomized Clinical Trial versus Anterior Cervical Discectomy and Fusion. The Spine Journal, 2007. 7(5S): p. 3.

34. Heller, J.G., et al., Comparison of BRYAN cervical disc arthroplasty with anterior cervical decompression and fusion: clinical and radiographic results of a randomized, controlled, clinical trial. Spine (Phila Pa 1976), 2009. 34(2): p. 101-7.

35. Powell, J.W., et al., Quality of Spinal Motion With Cervical Disk Arthroplasty: Computer-aided Radiographic Analysis. J Spinal Disord Tech, 2010.

36. Riew, K.D., et al., Cervical disc arthroplasty compared with arthrodesis for the treatment of myelopathy. J Bone Joint Surg Am, 2008. 90(11): p. 2354-64.

37. Riina, J., et al., The effect of an anterior cervical operation for cervical radiculopathy or myelopathy on associated headaches. Journal of Bone \& Joint Surgery - American Volume, 2009. 91(8): p. 1919-1923.

38. Sasso, R.C., et al., Results of cervical arthroplasty compared with anterior discectomy and fusion: four-year clinical outcomes in a prospective, randomized controlled trial. Orthopedics, 2011. 34(11): p. 889.

39. Sasso, R.C., et al., Results of cervical arthroplasty compared with anterior discectomy and fusion: four-year clinical outcomes in a prospective, randomized controlled trial. J Bone Joint Surg Am, 2011. 93(18): p. 1684-92.

40. Sasso, R.C., et al., Sagittal alignment after Bryan cervical arthroplasty. Spine (Phila Pa 1976), 2011. 36(13): p. 991-6.

41. Sasso, R.C. and N.M. Best, Cervical kinematics after fusion and bryan disc arthroplasty. Journal of spinal disorders \& techniques, 2008. 21(1) :(pp 19-22), 2008. Date of Publication: Feb 2008.): p. 22.

42. Sasso, R.C., et al., Motion analysis of bryan cervical disc arthroplasty versus anterior discectomy and fusion: results from a prospective, randomized, multicenter, clinical trial. J Spinal Disord Tech, 2008. 21(6): p. 393 9. 
43. Sasso, R.C., et al., Cervical disc replacement: Four-year follow-up results from the united states prospective randomized bryan clinical trial. Spine, 2009. Conference: 37th Annual Meeting of the Cervical Spine Research Society, CSRS 2009 Salt Lake City, UT United States. Conference Start: 20091203 Conference End: 20091205. Conference Publication:(var.pagings). , 2009. Date of Publication: 2009.).

44. Sasso, R.C., et al., Artificial disc versus fusion: a prospective, randomized study with 2-year follow-up on 99 patients. Spine (Phila Pa 1976), 2007. 32(26): p. 2933-40; discussion 2941-2.

45. Sasso, R.C., et al., Clinical outcomes of BRYAN cervical disc arthroplasty: a prospective, randomized, controlled, multicenter trial with 24-month follow-up. J Spinal Disord Tech, 2007. 20(7): p. 481-91.

46. Steinmetz, M.P., et al., Cervical disc arthroplasty compared with fusion in a workers' compensation population. Neurosurgery, 2008. 63(4) :(pp 741-747), 2008. Date of Publication: October 2008.): p. 747.

47. Delamarter, R.B., et al., Results at 24 months from the prospective, randomized, multicenter Investigational Device Exemption trial of ProDisc-C versus anterior cervical discectomy and fusion with 4-year follow-up and continued access patients. SAS J, 2010. 4(4): p. 122-8.

48. Kearns, S., et al., Five-Year Results of the Prodisc-C Multicenter Randomized Clinical Trial. The Spine Journal, 2011. 11(10): p. S46.

49. Kelly, M.P., et al., Adjacent Segment Motion Following Anterior Cervical Discectomy and Fusion versus ProDisc(R)-C Cervical Total Disk Arthroplasty: Analysis from a Randomized, Controlled Trial. Spine (Phila Pa 1976), 2011.

50. Kelly, M.P., et al., Comparison of adjacent segment motion following ACDF vs prodisc -C arthroplasty: Analysis from a randomized, controlled trial with 2 years follow-up. Spine, 2009. Conference: 37th Annual Meeting of the Cervical Spine Research Society, CSRS 2009 Salt Lake City, UT United States. Conference Start: 20091203 Conference End: 20091205. Conference Publication:(var.pagings). , 2009. Date of Publication: 2009.).

51. Murrey, D., et al., 5-Year results of the prospective, randomized, multi-center FDA Investigational Device Exemption (IDE) ProDisc-C TDR clinical trial. Spine, 2009. Conference: 37th Annual Meeting of the Cervical Spine Research Society, CSRS 2009 Salt Lake City, UT United States. Conference Start: 20091203 Conference End: 20091205. Conference Publication:(var.pagings). , 2009. Date of Publication: 2009.).

52. Murrey, D., et al., Results of the prospective, randomized, controlled multicenter Food and Drug Administration investigational device exemption study of the ProDisc-C total disc replacement versus anterior discectomy and fusion for the treatment of 1-level symptomatic cervical disc disease. Spine J, 2009. 9(4): p. 275-86.

53. Murrey, D.B., et al., Two-Year Results of a Randomized Controlled Clinical Trial Comparing ProDisc-C and Anterior Cervical Discectomy and Fusion. SAS Journal, 2008. 2(2) :(pp 76-85), 2008. Date of Publication: 2008.): p. 85

54. Park, J.J., et al., Analysis of segmental cervical spine vertebral motion after prodisc-C cervical disc replacement. Spine, 2010. 35(8) :(pp E285-E289), 2010. Date of Publication: April 2010.): p. E289.

55. Segebarth, B., et al., Incidence of dysphagia comparing cervical arthroplasty and ACDF. SAS J, 2010. 4(1): p. 3-8.

56. Marzluff, J., et al., 2-Year Multicenter Follow-up in a Prospective Randomized Clinical Trial: Comparison of a Cervical Artificial Disc to an ACDF Treatment. The Spine Journal, 2010. 10(9): p. S135-S136.

57. Howell, K., et al., A Prospective, Randomized Clinical Investigation of the Porous Coated Motion (PCM) Artificial Cervical Disc: Two-Year Results from the US IDE Study. The Spine Journal, 2011. 11(10, Supplement): p. S18.

58. McAfee, P.C., et al., Lower incidence of dysphagia with cervical arthroplasty compared with ACDF in a prospective randomized clinical trial. J Spinal Disord Tech, 2010. 23(1): p. 1-8.

59. Park, D.K., E.L. Lin, and F.M. Phillips, Index and adjacent level kinematics after cervical disc replacement and anterior fusion: in vivo quantitative radiographic analysis. Spine (Phila Pa 1976), 2011. 36(9): p. 721 30.

60. Burkus, J.K., et al., Long-term clinical and radiographic outcomes of cervical disc replacement with the Prestige disc: results from a prospective randomized controlled clinical trial. J Neurosurg Spine, 2010. 13(3): p. 308-18. 
61. Haid, R.W., et al. Long-term clinical and radiographic outcomes of the PRESTIGE ST cervical disc replacement: results from a prospective randomized controlled clinical trial. in 37th Annual Meeting of the Cervical Spine Research Society. 2009. Salt Lake City, UT.

62. Mummaneni, P.V., et al., Clinical and radiographic analysis of cervical disc arthroplasty compared with allograft fusion: a randomized controlled clinical trial. J Neurosurg Spine, 2007. 6(3): p. 198-209.

63. Riina, J., et al., Comparison of single-level cervical fusion and a metal-on-metal cervical disc replacement device. Am J Orthop, 2008. 37(4): p. E71-7.

64. Ahlhelm, F., et al., Segmentale Beweglichkeit nach anteriorer zervikaler Dekompression - Eine Vergleichsstudie., in Schweizer Archiv fur Neurologie und Psychiatrie. 2010: Basel.

65. Nabhan, A., et al., Disc replacement using Pro-Disc $C$ versus fusion: A prospective randomised and controlled radiographic and clinical study. European Spine Journal, 2007. 16(3) :(pp 423-430), 2007. Date of Publication: Mar 2007.): p. 430.

66. Nabhan, A., et al., The ProDisc-C prosthesis: clinical and radiological experience 1 year after surgery. Spine (Phila Pa 1976), 2007. 32(18): p. 1935-41.

67. Nabhan, A., et al., Assessment of adjacent-segment mobility after cervical disc replacement versus fusion: RCT with 1 year's results. Eur Spine J, 2011. 20(6): p. 934-41.

68. Nabhan, A., et al., Cervical spine disc replacement versus fusion: A prospective randomized and controlled radiographic and clinical study eurospine 2006. 8Th annual meeting of the european spine society, 25-28 october 2006, istanbul, turkey-abstracts \#41. Eur Spine J, 2006. 15(Suppl 4): p. S473.

69. Nabhan, A., et al., Segmental kinematics and adjacent level degeneration following disc replacement versus fusion: RCT with three years of follow-up. Journal of Long-Term Effects of Medical Implants, 2007. 17(3) :(pp 229-236), 2007. Date of Publication: 2007.): p. 236.

70. Pettine, K.A., Clinical and Radiographic Outcome of the NeoDisc Cervical Total Disc Replacement (TDR) at 2-Year Follow-up. The Spine Journal, 2010. 10(9): p. S44.

71. Brotchi, J., et al., A multicenter, prospective, randomized, study of an artificial disc versus fusion for primary cervical disc surgery. Orthopaedic Proceedings, 2004. 86-B(SUPP_I): p. 85-86.

72. McCombe, P., et al., A multicenter, prospective, randomized, study of the Prestige artificial disc versus fusion for primary cervical disc surgery. Orthopaedic Proceedings, 2005. 87-B(SUPP_III): p. 411-411.

73. Porchet, F. and N.H. Metcalf, Clinical outcomes with the Prestige II cervical disc: preliminary results from a prospective randomized clinical trial. Neurosurg Focus, 2004. 17(3): p. E6.

74. Porchet, F., et al., A multicenter, prospective, randomized, study of an artificial cervical disc versus fusion for primary cervical disc surgery, in 4th Annual Meeting of the Spine Society of Europe. 2002: Nantes, France.

75. Bhadra, A.K., et al., Single-level cervical radiculopathy: clinical outcome and cost-effectiveness of four techniques of anterior cervical discectomy and fusion and disc arthroplasty. Eur Spine J, 2009. 18(2): p. 232-7.

76. Bryan, V.E., Cervical motion segment replacement. European Spine Journal, 2002. 11(SUPPL. 2) :(pp S92S97), 2002. Date of Publication: 2002.): p. S97.

77. Cao, J., et al., Contrastive analysis of neck axial symptoms after Bryan cervical disc arthroplasty or traditional anterior cervical discectomy and fusion. [Chinese]. Zhongguo xiu fu chong jian wai ke za zhi = Zhongguo xiufu chongjian waike zazhi = Chinese journal of reparative and reconstructive surgery, 2008. 22(10) :(pp 1200-1204), 2008. Date of Publication: Oct 2008.): p. 1204.

78. Kim, S.W., et al., Comparison of radiographic changes after ACDF versus Bryan disc arthroplasty in single and bi-level cases. European Spine Journal, 2009. 18(2) :(pp 218-231), 2009. Date of Publication: February 2009.): p. 231.

79. Ogon, M., et al., Die Bandscheibenprothese an der Halswirbelsäule. J Fur Minerallstoffwechsel, 2006. 13: p. 14-17.

80. Park, J.H., et al., Comparative analysis of cervical arthroplasty using Mobi-C and anterior cervical discectomy and fusion using the Solis-cage. Journal of Korean Neurosurgical Society, 2008. 44(4) :(pp 217-221), 2008. Date of Publication: 2008.): p. 221.

81. Wang, Y., et al., [Clinical outcomes of single level Bryan cervical disc arthroplasty: a prospective controlled study]. Zhonghua Wai Ke Za Zhi, 2008. 46(5): p. 328-32. 
82. Peng-Fei, S. and J. Yu-Hua, Cervical disc prosthesis replacement and interbody fusion: a comparative study. Int Orthop, 2008. 32(1): p. 103-6.

83. Abitbol, J.-J., et al., 2-Year Results from Four IDE Study Sites: CerviCore Intervertebral Disc vs. Fusion. The Spine Journal, 2010. 10(9): p. S139-S140.

84. Abitbol, J.J., et al., CerviCore disc replacement versus fusion for single-level cervical radiculopathy: oneyear outcomes from 4 study sites in a prospective randomized controlled trial. Canadian Journal of Surgery, 2009. 52(3 Suppl): p. S7.

85. Attabib, N., et al., Cervical disc arthroplasty versus anterior cervical discectomy and fusion: prospective randomized trial. Canadian Journal of Surgery, 2008. 51(3 Suppl): p. S7-S8.

86. Bae, H.W., et al., Mobi-C Cervical Disc Replacement for the Treatment of One- and Two-Level Cervical Degenerative Disc Disease: One-Site Analysis Participating in the US FDA Trial. The Spine Journal, 2010. 10(9, Supplement 1): p. S145-S145.

87. Davis, R., et al., A prospective, randomised, controlled, multi-centre clinical study (IDE) comparing the Mobi-C cervical disc prosthesis with anterior discectomy and fusion in the treatment of degenerative cervical disc disease at 2 levels, in SFCR congress 2011 (French Spine Society). 2011, Springer-Verlag. p. 1209.

88. Davis, R., Comparison of the Mobi-C Cervical Artificial Disc to Anterior Cervical Discectomy and Fusion in the Treatment of Symptomatic Cervical Degenerative Disc Disease at Two Levels. The Spine Journal, 2011. 11(10): p. S135.

89. Nunley, P., et al., A Prospective Randomized Controlled Trial to Assess the Efficacy of the MobiC<sup>\&\#xae;</sup> Artificial Cervical Disc in the Management of Intractable Cervical Myelo-Radiculopathy at One or Two Contiguous Level. The Spine Journal, 2011. 11(10): p. S47-S48.

90. Bartels, R.H., et al., Design of the PROCON trial: a prospective, randomized multi-center study comparing cervical anterior discectomy without fusion, with fusion or with arthroplasty. BMC Musculoskelet Disord, 2006. 7: p. 85.

91. Buchowski, J.M., et al., Cervical disc arthroplasty compared with arthrodesis for the treatment of myelopathy. Surgical technique. J Bone Joint Surg Am, 2009. 91 Suppl 2: p. 223-32.

92. Robertson, J.T., S.M. Papadopoulos, and V.C. Traynelis, Assessment of adjacent-segment disease in patients treated with cervical fusion or arthroplasty: a prospective 2-year study. J Neurosurg Spine, 2005. 3(6): p. 417-23.

93. Guyatt, G.H., et al., GRADE guidelines: 4. Rating the quality of evidence--study limitations (risk of bias). J Clin Epidemiol, 2011. 64(4): p. 407-15.

94. Cepoiu-Martin, M., et al., Artificial cervical disc arthroplasty: a systematic review. Spine (Phila Pa 1976), 2011. 36(25): p. E1623-33.

95. Jiang, H., et al., Cervical disc arthroplasty versus fusion for single-level symptomatic cervical disc disease: a meta-analysis of randomized controlled trials. Arch Orthop Trauma Surg, 2012. 132(2): p. 141-51.

96. McAfee, P.C., et al., A meta-analysis of comparative outcomes following cervical arthroplasty or anterior cervical fusion: results from 4 prospective multicenter randomized clinical trials and up to 1226 patients. Spine (Phila Pa 1976), 2012. 37(11): p. 943-52.

97. Upadhyaya, C.D., et al., Analysis of the three United States Food and Drug Administration investigational device exemption cervical arthroplasty trials. J Neurosurg Spine, 2012. 16(3): p. 216-28.

98. Yu, L., et al., Systematic review and meta-analysis of randomized controlled trials: comparison of total disk replacement with anterior cervical decompression and fusion. Orthopedics, 2011. 34(10): p. e651-8. 


\section{Chapter 2 - Appendix 1 - Search strategies}

\section{CENTRAL search strategy}

\#1 MeSH descriptor Neck explode all trees

\#2 MeSH descriptor Neck Pain explode all trees

\#3 MeSH descriptor Cervical Vertebrae explode all trees

\#4 (cerv* NEAR/5 (spine or spinal))

\#5 (cerv* NEAR/5 (disc* or disk*))

\#6 ACDF

\#7 (\#1 OR \#2 OR \#3 OR \#4 OR \#5 OR \#6)

\#8 MeSH descriptor Spinal Fusion explode all trees

\#9 (spine* or spinal) NEAR/2 fusion

\#10 cerv* NEAR/5 fusion

\#11 MeSH descriptor Diskectomy explode all trees

\#12 discectomy

\#13 diskectomy

\#14 MeSH descriptor Intervertebral Disk explode all trees

\#15 MeSH descriptor Intervertebral Disk Displacement explode all trees

\#16 slipped NEAR/2 (disc* or disk*)

\#17 degenerat* NEAR/2 (disc* or disk*)

\#18 displace* NEAR/2 (disc* or disk*)

\#19 herniat* NEAR/2 (disc* or disk*)

\#20 MeSH descriptor Radiculopathy explode all trees

\#21 MeSH descriptor Spondylosis explode all trees

\#22 MeSH descriptor Spinal Osteophytosis explode all trees

\#23 (spine* or spinal) NEAR/2 decompress*

\#24 MeSH descriptor Decompression, Surgical explode all trees

\#25 (anterior or posterior) NEAR/2 fusion

\#26 fixation NEAR/2 (spine* or spinal)

\#27 stabili* NEAR/2 (spine* or spinal)

\#28 (\#8 OR \#9 OR \#10 OR \#11 OR \#12 OR \#13 OR \#14 OR \#15 OR \#16 OR \#17 OR \#18 OR

\#19 OR \#20 OR \#21 OR \#22 OR \#23 OR \#24 OR \#25 OR \#26 OR \#27)

\#29 (\#7 AND \#28)

\#30 MeSH descriptor Arthroplasty explode all trees

\#31 (spine* or spinal) NEAR/2 arthroplasty

\#32 cerv* NEAR/2 arthroplasty

\#33 MeSH descriptor Arthroplasty, Replacement explode all trees

\#34 Diskectomy

\#35 diskectomy

\#36 MeSH descriptor Diskectomy explode all trees 
\#37 MeSH descriptor Intervertebral Disk explode all trees

\#38 MeSH descriptor Intervertebral Disk Displacement explode all trees

\#39 degenerat* NEAR/2 (disc* or disk*)

\#40 slipped NEAR/2 (disc* or disk*)

\#41 displace* NEAR/2 (disc* or disk*)

\#42 herniat* NEAR/2 (disc* or disk*)

\#43 MeSH descriptor Radiculopathy explode all trees

\#44 MeSH descriptor Spondylosis explode all trees

\#45 MeSH descriptor Spinal Osteophytosis explode all trees

\#46 (spine* or spinal) NEAR/2 decompress*

\#47 MeSH descriptor Decompression, Surgical explode all trees

\#48 fixation NEAR/2 (spine* or spinal)

\#49 stabili* NEAR/2 (spine* or spinal)

\#50 MeSH descriptor Joint Prosthesis explode all trees

\#51 ((disk* or disc*) NEAR/2 (replac* or prosthes*))

\#52 (\#30 OR \#31 OR \#32 OR \#33 OR \#34 OR \#35 OR \#36 OR \#37 OR \#38 OR \#39 OR \#40

OR \#41 OR \#42 OR \#43 OR \#44 OR \#45 OR \#46 OR \#47 OR \#48 OR \#49 OR \#50 OR \#51)

\#53 (\#7 AND \#52)

\#54 (\#29 OR \#53)

\section{MEDLINE search strategy}

1. randomized controlled trial.pt.

2. controlled clinical trial.pt.

3. randomized.ab.

4. placebo.ab,ti.

5. drug therapy.fs.

6. randomly.ab,ti.

7. trial.ab,ti.

8. groups.ab,ti.

9. or/1-8

10. (animals not (humans and animals)).sh.

11. 9 not 10

12. Neck/

13. exp Neck Pain/

14. Cervical Vertebrae/

15. (cerv* adj5 (spine or spinal)).mp

16. (cerv* adj5 (disc* or disk*)).mp.

17. ACDF.mp.

18. or/12-17

19. exp Spinal Fusion/ 
20. ((spine* or spinal) adj2 fusion).mp.

21. (cerv* adj5 fusion).mp.

22. exp Diskectomy/

23. discectomy.mp.

24. diskectomy.mp.

25. Intervertebral Disk/

26. Intervertebral Disk Displacement/

27. (slipped adj2 (disc* or disk*)).mp.

28. (degenerat* adj2 (disc* or disk*)).mp.

29. (displace* adj2 (disc* or disk*)).mp.

30. (herniat* adj2 (disc* or disk*)).mp.

31. Radiculopathy/

32. Spondylosis/

33. Spinal Osteophytosis/

34. ((spine* or spinal) adj2 decompress*).mp.

35. Decompression, Surgical/

36. ((anterior or posterior) adj2 fusion).mp.

37. (fixation adj2 (spine* or spinal)).mp.

38. (stabili* adj2 (spine* or spinal)).mp.

39. or/19-38

40. 18 and 39

41. Arthroplasty/

42. ((spine* or spinal) adj2 arthroplasty).mp.

43. (cerv* adj2 arthroplasty).mp.

44. exp Arthroplasty, Replacement/

45. exp Diskectomy/

46. discectomy.mp.

47. diskectomy.mp.

48. Intervertebral Disk/

49. Intervertebral Disk Displacement/

50. (slipped adj2 (disc* or disk*)).mp.

51. (degenerat* adj2 (disc* or disk*)).mp.

52. (displace* adj2 (disc* or disk*)).mp.

53. (herniat* adj2 (disc* or disk*)).mp.

54. Radiculopathy/

55. Spondylosis/

56. Spinal Osteophytosis/

57. ((spine* or spinal) adj2 decompress*).mp.

58. Decompression, Surgical/

59. (fixation adj2 (spine* or spinal)).mp.

60. (stabili* adj2 (spine* or spinal)).mp. 
61. Joint Prosthesis/

62. ((disk* or disc*) adj2 (replac* or prosthes*)).mp.

63. or/41-62

64. 18 and 63

65.40 or 64

66. 11 and 65

\section{EMBASE search strategy}

1. Clinical Article/

2. exp Clinical Study/

3. Clinical Trial/

4. Controlled Study/

5. Randomized Controlled Trial/

6. Major Clinical Study/

7. Double Blind Procedure/

8. Multicenter Study/

9. Single Blind Procedure/

10. Phase 3 Clinical Trial/

11. Phase 4 Clinical Trial/

12. crossover procedure/

13. placebo/

14. or/1-13

15. allocat\$.mp.

16. assign\$.mp.

17. blind\$.mp.

18. (clinic\$ adj25 (study or trial)).mp.

19. compar\$.mp.

20. control\$.mp.

21. cross?over.mp.

22. factorial\$s.mp.

23. follow?up.mp.

24. placebo\$.mp.

25. prospectiv\$.mp.

26. random\$.mp.

27. ((singl\$ or doubl\$ or trebl\$ or tripl\$s) adj25 (blind\$ or mask\$)).mp.

28. trial.mp.

29. (versus or vs).mp.

30. or/15-29

31. 14 and 30

32. human/ 
33. Nonhuman/

34. $\exp$ ANIMAL/

35. Animal Experiment/

36.33 or 34 or 35

37. 32 not 36

38. 31 not 36

39. 37 and 38

40. 38 or 39

41. neck/

42. cervical spine/

43. neck pain/

44. (cerv* adj5 (spine or spinal)).mp.

45. (cerv* adj5 (disc* or disk*)).mp.

46. ACDF.mp.

47. or/41-45

48. exp spine fusion/

49. ((spine* or spinal) adj2 fusion).mp.

50. (cerv* adj5 fusion).mp.

51. discectomy.mp.

52. intervertebral diskectomy/

53. diskectomy.mp.

54. intervertebral disk/

55. intervertebral disk hernia/

56. (slipped adj2 (disc* or disk*)).mp.

57. (degenerat* adj2 (disc* or disk*)).mp.

58. (displace* adj2 (disc* or disk*)).mp.

59. (herniat* adj2 (disc* or disk*)).mp.

60. exp radiculopathy/

61. cervical spondylosis/

62. ((spine* or spinal) adj2 decompress*).mp.

63. decompression surgery/

64. ((anterior or posterior) adj2 fusion).mp.

65. (fixation adj2 (spine* or spinal)).mp.

66. (stabili* adj2 (spine* or spinal)).mp.

67. or/48-66

68. discectomy.mp.

69. intervertebral diskectomy/

70. diskectomy.mp.

71. intervertebral disk/

72. intervertebral disk hernia/

73. (slipped adj2 (disc* or disk*)).mp. 
74. (degenerat* adj2 (disc* or disk*)).mp.

75. (displace* adj2 (disc* or disk*)).mp.

76. (herniat* adj2 (disc* or disk*)).mp.

77. exp radiculopathy/

78. cervical spondylosis/

79. ((spine* or spinal) adj2 decompress*).mp.

80. decompression surgery/

81. (fixation adj2 (spine* or spinal)).mp.

82. (stabili* adj2 (spine* or spinal)).mp.

83. arthroplasty/

84. ((spine* or spinal) adj2 arthroplasty).mp.

85. (cerv* adj2 arthroplasty).mp.

86. joint prosthesis/

87. ((disk* or disc*) adj2 (replac* or prosthes*)).mp.

88. or/68-87

89. 47 and 67

90.47 and 88

91. 89 or 90

92. 40 and 91

Trials Register search strategy

((surg* OR fusion) AND cervical)

Search all non-indexed text fields 


\section{Chapter 2 - Appendix 2 - Risk of Bias analysis}

\section{Allocation (selection bias)}

Four of the studies used adequate randomisation and allocation concealment procedures (Heller 2009; Marzluff 2010; McAfee 2010; Mummaneni 2007). One study used an adequate allocation concealment procedure, but the method of randomisation was unclear (Kelly 2011). One study used an adequate randomisation procedure, but the allocation concealment procedure was unclear (Porchet 2004). For the other three studies both the randomisation and allocation concealment procedures were unclear (Coric 2011; Nabhan 2007; Pettine 2010).

We identified no significant differences between the arthroplasty groups and fusion groups at baseline for the primary outcomes with a baseline measurement. VAS arm pain $(P=0.99)$, VAS neck pain $(P=0.50), N D I(P=0.64)$, Short Form-36 Physical Health Component Score (SF36-PCS) $(P=0.26)$, Short Form-36 Mental Health Component Score (SF36-MCS) $(P=0.23)$. This supports overall adequate randomisation.

\section{Blinding (performance bias and detection bias)}

There were no studies that were blinded for either patient, care provider, or outcome assessor at all time points. Five studies were explicitly reported to not be blinded (Heller 2009; Kelly 2011; Marzluff 2010; McAfee 2010; Mummaneni 2007); for the other four studies blinding was unclear (Coric 2011; Nabhan 2007; Pettine 2010; Porchet 2004).

\section{Incomplete outcome data (attrition bias)}

Seven studies had sufficient levels of follow-up to qualify for a low risk of bias (Coric 2011; Heller 2009; Kelly 2011; Marzluff 2010; McAfee 2010; Mummaneni 2007; Pettine 2010). One study had insufficient levels of follow-up at three months (Nabhan 2007), which was primarily because of patients that were excluded from the analysis due to radiographic markers being obscured. However, this should not be a reason to exclude these patients from other, non-radiographic, outcome measures. For one study the actual level of follow-up was unclear, since they reported data for participants evaluated at the one-year time point at the time writing the publication, without stating how many participants were not yet at the one-year time point (Porchet 2004).

\section{Selective reporting (reporting bias)}

Six studies were judged to have a low risk of selective outcome reporting (Coric 2011; Heller 2009; Kelly 2011; Marzluff 2010; Mummaneni 2007; Pettine 2010). Three studies were judged to have a high risk of bias on this subject (McAfee 2010; Nabhan 2007; Porchet 2004). For one of these studies primary outcome scores (e.g. VAS arm pain, VAS neck 
pain, NDI) were not reported for the entire population (McAfee 2010). For the second study it was reported in one of the abstracts that the NDI was used, but results on this outcome parameter were never reported, although three full-text articles concerning this study population have been published to date (Nabhan 2007). For the third study it was reported that neurological outcome was measured, but results for this outcome were not reported (Porchet 2004).

\section{Publication bias}

We planned to use funnel plots to investigate publication bias. Since we included nine studies, the recommended minimum number of 10 studies could not be met for any of the outcomes (Higgins 2011). Given the number of ongoing trials, this should be possible in a future update of this review.

\section{Sponsoring/conflict of interest}

Almost all of the included studies (eight of nine) were industry sponsored, and in many cases one or more of the authors/investigators had a conflict of interest. Given the logistics and costs involved when conducting a large-scale surgical trial, as is demanded by the FDA in order to acquire approval to use a medical device in the US, it is almost impossible for investigators to conduct such a trial without industry sponsoring. We did receive the complete study data for several trials from the sponsor (Heller 2009; Mummaneni 2007; Porchet 2004). In one case we received data for the entire study population although results for only a subset of the participating centres had been published at that time (Marzluff 2010).

Conflict of interest of the investigators can be a source of bias. Although most of the primary outcomes were patient reported it is important to realise that patients' appreciations can be influenced by the treating physician (e.g. patient expectations were based on the information provided).

The influence of conflict of interest of investigators was investigated for two studies (Heller 2009; Mummaneni 2007) by comparing results from investigators with a financial interest during some point of the clinical study ( $22 \%$ to $28 \%$ of the investigators and coinvestigators) with the other investigators. There were no statistical differences in one study. In the other study NDI and overall success rate were significantly lower in the investigational group from investigators with a conflict of interest, at one year' follow-up. These analyses were reported in the clinical study report that was available through the FDA website, but was not mentioned elsewhere. Given the amount of discussion on this subject it would be interesting if this type of analysis could be reported standardly in future reports. 


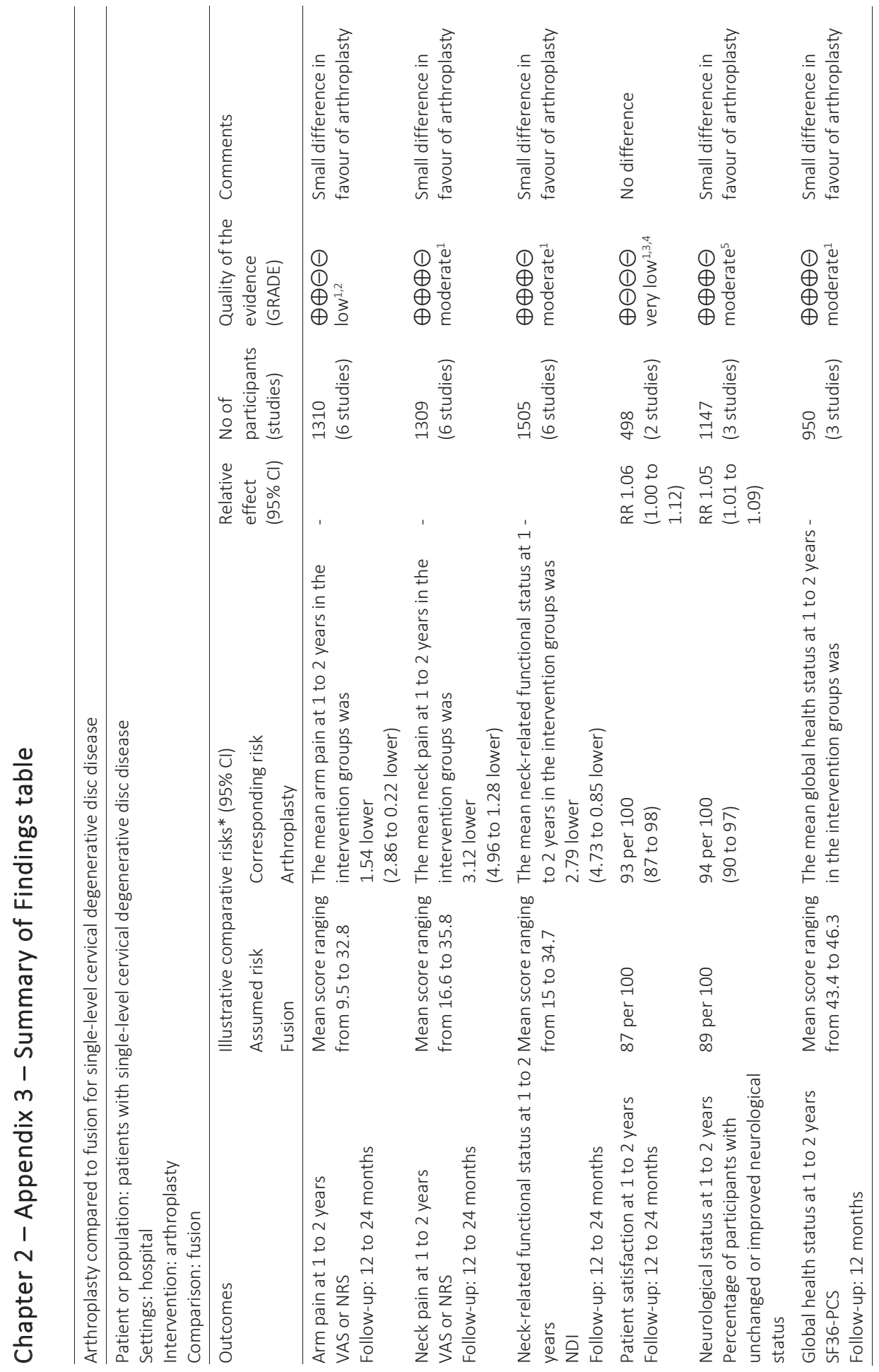




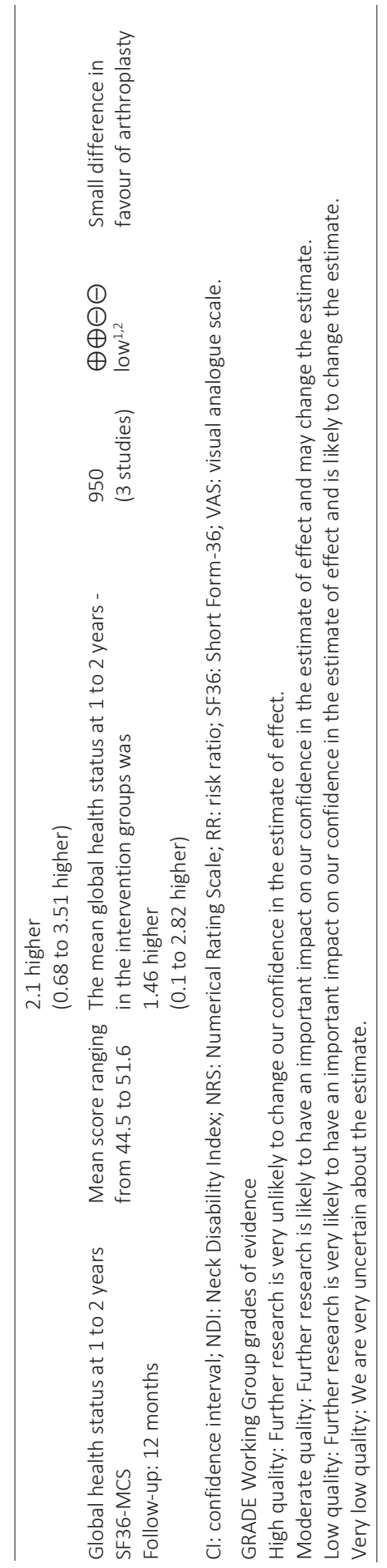





\section{Chapter}

\section{Cervical Vertebrae Tracking in Video-Fluoroscopy Using the Normalized Gradient Field}

Rianne Reinartz, Bram Platel, Toon Boselie, Henk van Mameren, Henk van Santbrink, Bart ter Haar Romeny Medical Image Computing and Computer-Assisted Intervention 2009 


\begin{abstract}
For patients with neck problems valuable functional and diagnostic information can be obtained from a fluoroscopy video of a flexion-extension movement of the cervical spine. In most cases physicians have to manually extract the vertebrae, making the analysis of these video sequences tedious and time consuming. In this paper we propose an automatic fast and precise method for tracking cervical vertebrae. Our method relies only on a rough selection of template areas of each vertebra in a single frame of the video sequence. Compared to existing automated methods, no contours need to be extracted and no vertebra segmentation is required. Tracking is done with a normalized gradient field, using only the gradient orientations as features. Experimental results show that the algorithm is robust and able to track the vertebrae accurately even if they are partially occluded or if a disc prosthesis is present.
\end{abstract}




\section{Introduction}

The order in which the segments of the cervical spine contribute to the motion of the skull with respect to the thorax during anteflexion and retroflexion (forward and backward bending of the neck), has proven to provide valuable functional and diagnostic information for patients with neck complaints $[1,2]$. To analyse the movement of the cervical spine in detail, a video-fluoroscopy recording (i.e. an x-ray video), is created for a movement from maximum extension to maximum flexion of the neck (Figure 1). From this recording the rotation and translation of the cervical vertebrae with respect to each other need to be extracted. Automatic spinal measurement techniques have been studied for the lumbar as well as the cervical spine. Generally these methods are either based on landmark detection, or on the extraction of vertebral boundaries. Due to noise, occlusion and unclear vertebral contours, the detection of landmarks or contours is difficult. Techniques exploiting the Hough transform [3, 4], active appearance models [5], level sets [6], and polar signatures [7], have been used to find the unclear vertebral boundaries and track them in fluoroscopy videos. These methods often use large sets of templates to capture the great variability in the shape of the vertebrae, require extensive manual interaction, are computationally heavy and are complex to implement. For the motion analysis of the cervical spine, exact contour extraction and vertebra segmentation is not necessary. We therefore propose a method based on the normalized gradient field that can automatically track cervical vertebra in a video-fluoroscopy sequence, requiring user interaction in only a single frame of the video. This eliminates the cumbersome and computationally heavy segmentation of the vertebral contours. 


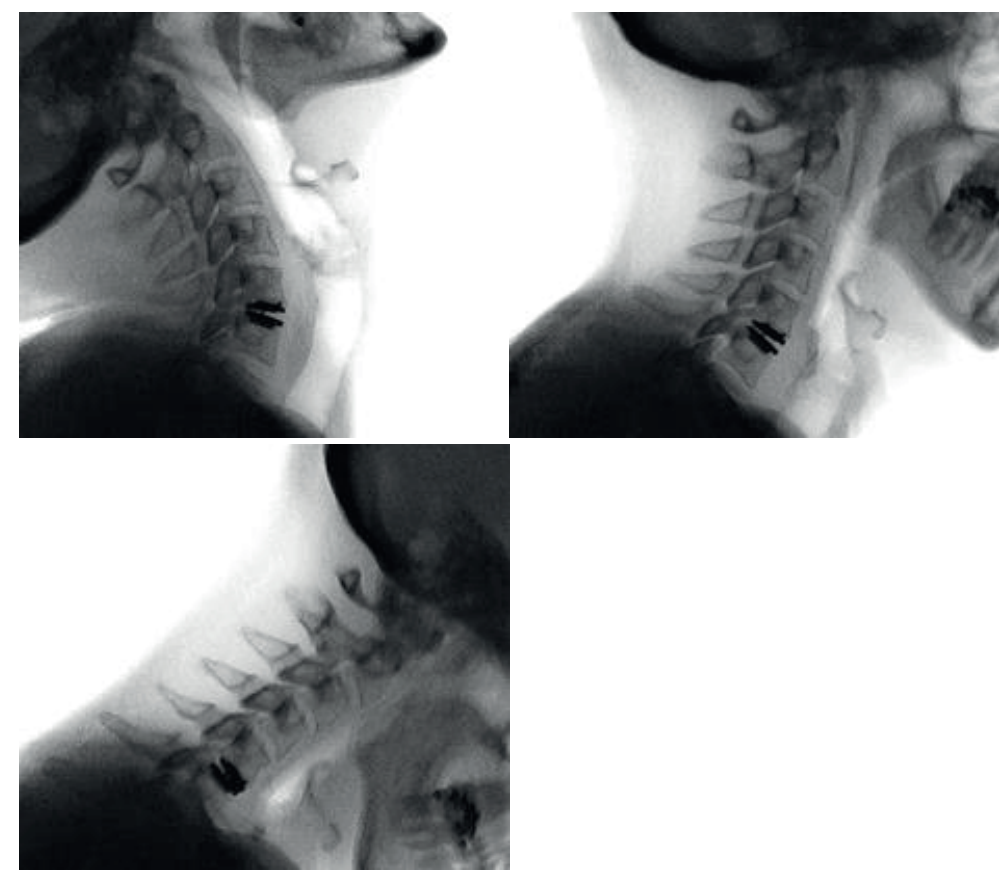

Figure 1: Three frames of a typical cervical fluoroscopy video. The images respectively show the patient in extension (retroflexion), neutral position and anteflexion.

\section{Methods}

\subsection{Cervical Video-Fluoroscopy}

To acquire the cervical fluoroscopy video, the subject is placed on a stool, with his shoulders perpendicular to the image intensifier. The shoulder nearest to the image intensifier is placed directly against it. The subject is asked to move his head in maximal extension without moving the upper part of his body. As soon as the recording is started, the subject is instructed to move his head in the sagittal plane from maximal extension to maximal flexion, without moving the upper part of the body. The subjected is asked to perform the full movement in about 15 seconds. It is important that the subject's shoulders are kept as low as possible while making the cervical fluoroscopy videos to ensure that all the cervical vertebrae are visible. The movement of the cervical spine should be as fluent as possible to prevent for sudden large rotations and translations between consecutive frames. The fluoroscopic recordings were made with a digital X-ray detector, capturing frames of $1024 \times 1024$ pixels, at 10 frames per second. The recordings were stored without compression. 


\subsection{Template Selection}

The tracking algorithm requires a template of each vertebral body and the skull, which can be tracked throughout the whole video sequence. Due to partial occlusion by the shoulder of C5 and C6 only the anterior parts of these vertebrae are visible during retroflexion, which is why these anterior parts are taken as a template. The anterior parts of $\mathrm{C} 1$ and $\mathrm{C} 2$ appear as one, as the dens of C2 moves into C1 to form the atlanto-axial joint. This is why only the posterior parts of these vertebrae are used as a template. The bottom part of the maxillary sinus and the palatum durum are projected in the $\mathrm{x}$-ray image as a stable and structure rich area of the skull. This is therefore an ideal template to track the movement of CO (the skull). C7 is usually completely occluded by the shoulder and can therefore not be tracked. Figure 2 shows the template regions. The user is asked to draw these templates in a single frame of the fluoroscopy video. The templates are selected by positioning a closed spline over the template area. This spline is positioned by moving a small number of points (the user can choose the amount, but these are usually 4 points) over the image.

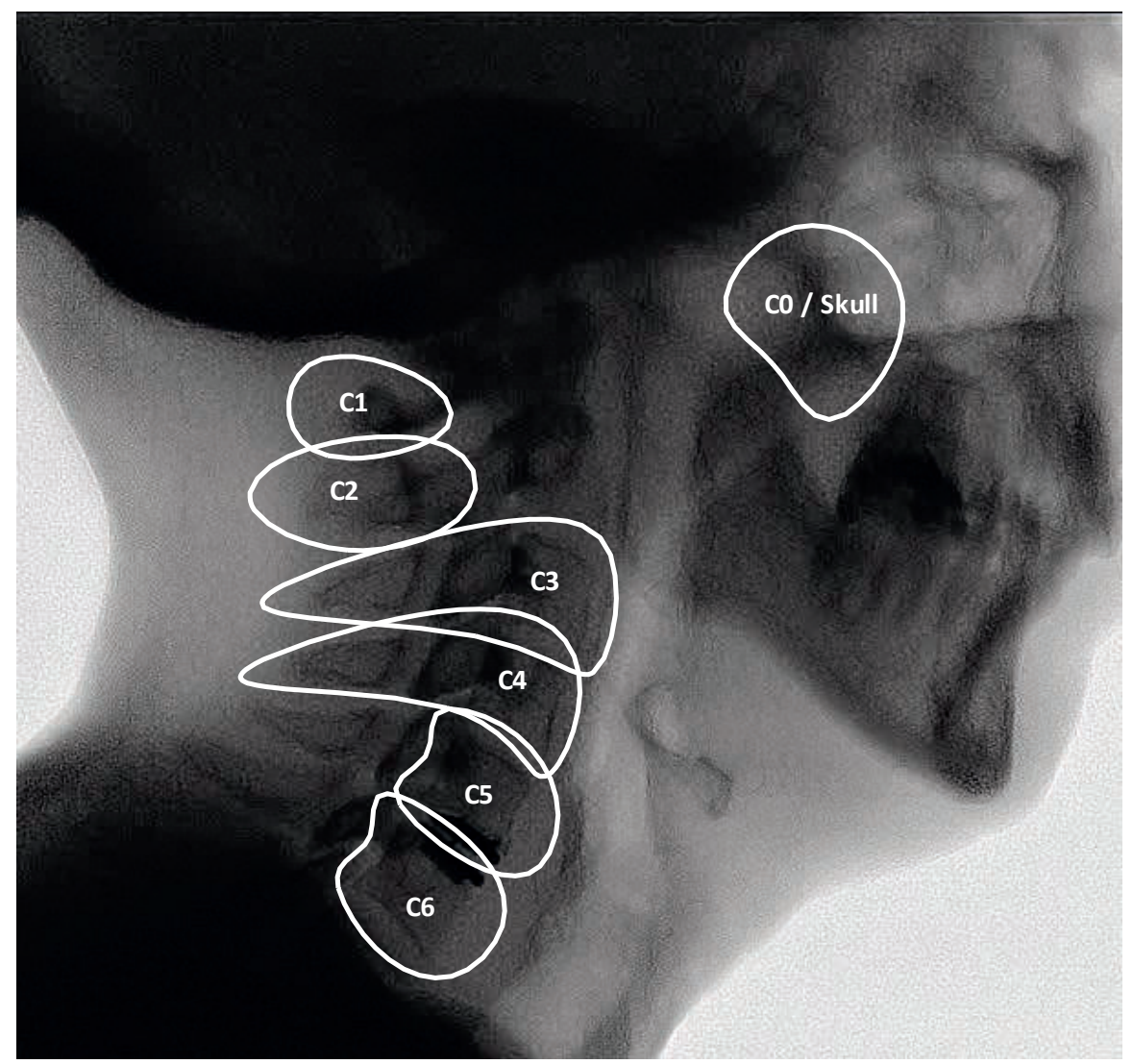

Figure 2: An example set of templates for the vertebrae and the skull. 


\subsection{Vertebra Tracking}

We define a user selected portion of a vertebral body as the template $T$, and the subsequent image in the fluoroscopy video sequence as the reference $R$. The goal is to find a rigid transformation $\phi$ such that the "distance" between the template $T$, and the reference $R$, is as small as possible. The rigid transformation is defined as:

$$
\phi(\gamma, p)=\left(\begin{array}{cc}
\cos \left(\gamma_{1}\right) & -\sin \left(\gamma_{1}\right) \\
\sin \left(\gamma_{1}\right) & \cos \left(\gamma_{1}\right)
\end{array}\right)\left(\begin{array}{l}
p_{1} \\
p_{2}
\end{array}\right)+\left(\begin{array}{c}
\gamma_{2} \\
\gamma_{3}
\end{array}\right)
$$

Given a distance measure $D$, the goal is to find a minimizer $\gamma$ of

$$
f(\gamma):=D(R(\cdot), T(\varphi(\gamma, \cdot)))
$$

The cervical vertebrae tracking is done on a video sequence of fluoroscopic images.

Partial occlusion of the vertebrae by other bones causes significant intensity variations. A proper distance measure to register the reference and target should therefore not depend on the intensities of the image. We have chosen to use the normalized gradient field (NGF) as described by Haber [8]. The NGF contains the local orientations of the image gradient. In regions where the intensities are nearly constant the gradient orientation is unreliable. To lower the weight of these gradient orientations a parameter $\epsilon$ is introduced so that the normalized gradient $\widehat{\nabla}_{\epsilon}$ becomes

$$
\hat{\nabla}_{\epsilon} I:=\frac{\nabla I}{\sqrt{|\nabla I|^{2}+\epsilon^{2}}}, \quad \text { with } \quad \epsilon=\frac{\eta}{A} \int_{\Omega}|\nabla I(p)| d p,
$$

where $\eta$ is the estimated noise level in the image and $A$ is the area of the image domain $\Omega$. With $S:=T \cdot \phi$, the normalized gradient vectors at position $p$ become $\left\{\hat{R}_{x}(p), \hat{R}_{y}(p)\right\}=\widehat{\nabla}_{\epsilon} R(p)$ and $\left\{\hat{S}_{x}(p), \hat{S}_{y}(p)\right\}=\widehat{\nabla}_{\epsilon} S(p)$. As the gradient fields are approximately normalized, the angle $\theta(p)$ between the vectors $\left\{\hat{R}_{x}(p), \hat{R}_{y}(p)\right\}$ and $\left\{\hat{S}_{x}(p), \hat{S}_{y}(p)\right\}$ is given by:

$$
\begin{aligned}
\left|\hat{R}_{x}(p) \hat{S}_{y}(p)-\hat{R}_{y}(p) \hat{S}_{x}(p)\right|= & \left|\left\{\hat{R}_{x}(p), \hat{R}_{y}(p)\right\}\right|\left|\left\{\hat{S}_{x}(p), \hat{S}_{y}(p)\right\}\right| \cdot \sin (\theta(p)) \\
& \approx \sin (\theta(p)) .
\end{aligned}
$$


As we want to minimize the differences in the angles between the normalized gradient field of reference $R$ and template $T$ we define the distance between two normalized gradient vectors as

$$
d(R(p), T(\varphi(p)))=\left(\left(\hat{R}_{x}(p) \hat{S}_{y}(p)-\hat{R}_{y}(p) \hat{S}_{x}(p)\right)^{2}\right.
$$

The overall distance between $R$ and $T$ is defined as

$$
\begin{aligned}
D(R, T) & =\frac{1}{2} \int_{\Omega} d(R(p), T(\varphi(p))) d p \\
& \approx \frac{h^{2}}{2} \sum_{p \in \omega}\left(\hat{R}_{x}(p) \hat{S}_{y}(p)-\hat{R}_{y}(p) \hat{S}_{x}(p)^{2} .\right.
\end{aligned}
$$

Where $h$ is the distance between grid points, defined as the image domain $\Omega$, divided by the number of grid points in each direction.

A Gauss-Newton optimization scheme is used to solve for the registration parameters $\gamma$. For each iteration the parameter change $s$ is found that minimizes $f(\gamma+s)$. Using a second order Taylor expansion we obtain

$$
f(\gamma+s) \approx f(\gamma)+\nabla f(\gamma) \cdot s+\frac{1}{2} s \cdot \nabla^{2} f(\gamma) \cdot s .
$$

This equation needs to be minimized; therefore the first order derivative of the function with respect to $s$ should equal zero.

$$
f^{\prime}(\gamma+s) \approx \nabla f(\gamma)+\nabla^{2} f(\gamma) \cdot s
$$

By setting the result equal to zero and rearranging the terms we obtain

$$
-\nabla f(\gamma)=\nabla^{2} f(\gamma) \cdot s
$$

$f(\gamma)$ can be written in a general form:

$$
f(\gamma)=\frac{h^{2}}{2}|r(\gamma)|_{2}^{2}, \text { with } \quad r\left(\gamma_{p}\right)=\hat{R}_{x}(p) \hat{S}_{y}(p)-\hat{R}_{y}(p) \hat{S}_{x}(p)
$$

The matrix of the first-order partial derivatives of $r$ is the Jacobian, 


$$
J=\left[\frac{\partial r_{j}}{\partial \gamma_{p}}\right]
$$

By using the Jacobian the derivatives of $f(\gamma)$ can be written as:

$$
\begin{gathered}
\nabla f(\gamma)=J(\gamma)^{T} r(\gamma) . \\
\nabla^{2} f(\gamma)=J(\gamma)^{T} J(\gamma)+\sum_{j=1}^{2} r_{j}(\gamma) \nabla^{2} r_{j}(\gamma) \approx J(\gamma)^{T} J(\gamma) .
\end{gathered}
$$

Substituting Eqn. 12 and 13 in Eqn. 9 and rearranging the terms leads to

$$
s=-\left(J(\gamma)^{T} J(\gamma)\right)^{-1} J(\gamma)^{T} r(\gamma)
$$

The partial derivates $\frac{\partial r}{\partial \gamma}$ of $r(T(\varphi(\gamma)))$ can be calculated using the chain rule

$$
\frac{\partial r}{\partial \gamma}=\frac{\partial r}{\partial T} \frac{\partial T}{\partial \varphi} \frac{\partial \varphi}{\partial \gamma}
$$

leading to

$$
\frac{\partial r}{\partial \gamma}=\left(-\hat{R}_{y} \hat{R}_{x}\right)\left(\begin{array}{c}
\hat{S}_{x x} \hat{S}_{x y} \\
\hat{S}_{x y} \hat{S}_{y y}
\end{array}\right)\left(\begin{array}{ccc}
-x_{1} \sin \left(\gamma_{1}\right)-x_{2} \cos \left(\gamma_{1}\right) & 1 & 0 \\
x_{1} \cos \left(\gamma_{1}\right)-x_{2} \sin \left(\gamma_{1}\right) & 0 & 1
\end{array}\right)
$$

We use a convolution with a Gaussian kernel to calculate image derivatives.

$$
\phi_{\sigma}(x, y)=\frac{1}{2 \pi \sigma^{2}} e^{-\frac{1}{2}\left(x^{2}+y^{2}\right) / \sigma^{2}} .
$$

Derivatives of the image can be calculated at any scale by

$$
D I(x, y, \sigma)=\left(D \varphi_{\sigma} * I\right)(x, y),
$$


where $D$ is any linear derivative operator with constant coefficients. By convolving the reference image $R$ with the first order derivatives of the Gaussian kernel we obtain $\hat{R}_{x}$ and $\hat{R}_{y}$ and similarly by convolving the transformed template $T$ with the second order derivatives of the Gaussian kernel we obtain $\hat{S}_{x x}, \hat{S}_{x y}$, and $\hat{S}_{y y}$.

By iterating Eqn. 14 and updating the parameters $\gamma_{i+1}=\gamma_{i}+s$ the best match between the template and the reference image is established. The process is repeated until the distance $D(R, T)$ divided by the number of pixels in the template $T$, between two consecutive iterations, is less than $1 e-9$. This threshold is experimentally set. A change of an order of magnitude in this threshold will not significantly change the outcome of the algorithm. This threshold is the same for all sequences.

The obtained parameters $\gamma$ can be used to track the cervical vertebra from frame $i$ to frame $i+1$. This is done for each vertebra and for the skull.

\section{Results}

The results of our automatic tracking algorithm are presented to the user in an animation as shown in Figure 3. The results were validated by comparison with manual vertebrae outline tracking by a trained clinician. The manual tracking was obtained by outlining all cervical vertebrae and the skull in a neutral frame.
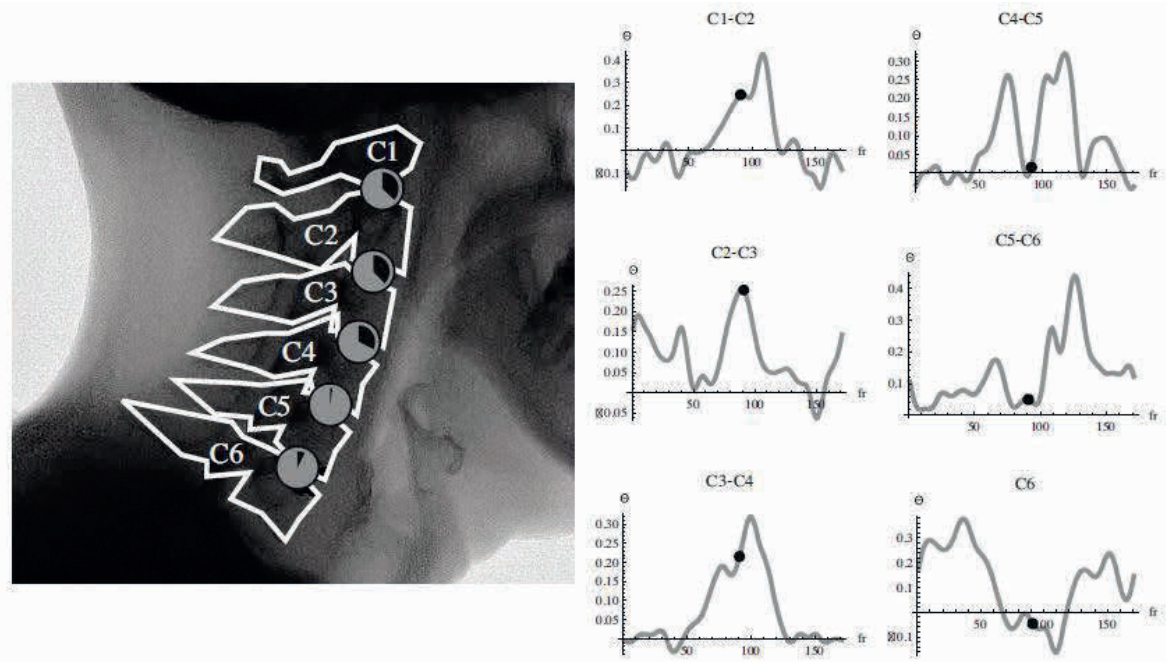

Figure 3: Visualization of the results; the user is presented with an animation where on the left side the vertebrae and a moving outline (user-specified in a single frame) are shown; the amount of rotation between two adjacent vertebrae is visualized by (exaggerated) polar-wedge diagrams. On the right side the amount of rotation between adjacent vertebrae is quantified in graphs, the black dot represents the current frame. 
In the consecutive frames the outlines of the previous frame are displayed over the image. These outlines are then rotated and translated horizontally and vertically by the user until they visually match with the corresponding vertebrae in that frame. This is repeated until all the frames contain matching outlines. This manual analysis of an entire anteflexion-flexion movie takes a trained expert 5 to 6 hours.

\section{Discussion}

The angles of rotation obtained by both the expert and our algorithm were compared for three separate cervical fluoroscopy video's, of which one contains a disc prosthesis. In all cases the automatic method performed as well as, or better than the manual tracking by the expert. The performance of one sequence is shown in Figure 4. It is clear that the differences are small apart from some manual annotation errors that are apparent as high peaks in the graphs. Our algorithm is robust to variations in the choice of the templates (Section 2.2) as long as a substantial part of template is visible throughout the entire sequence. If, however, the template area is too small, a region without any structure or an area which will be largely occluded later in the video sequence is selected, the algorithm will fail to track that vertebra. As a test we have asked six different users to draw the templates for the vertebrae and the skull, and in all of these cases the algorithm was able to track the vertebrae flawlessly, even though there were substantial differences in the selected areas.

Our method is insensitive for the presence of a disc prosthesis, which is valuable as it enables the analysis of the movement of the cervical spine before and after the placement of such a prosthesis.

The frames of the video sequence were cropped such that only areas that contain information on the vertebrae and the skull were kept, i.e. the shoulders were removed from the image and any excess space in front of or above the patient was removed. On average this reduces the dimensions of the images to $500 \times 500$ pixels. Implementing these changes into the fluoroscopy acquisition protocol will reduce the dose. Selecting the neutral frame and marking the template areas on the video sequence takes no more than 3 minutes and is all the user interaction required for the algorithm to run. Our Mathematica $^{\text {TM }}$ implementation of the automatic tracking algorithm takes around 45 minutes to run for all vertebrae on the entire fluoroscopy video sequence, this algorithm runs unsupervised. 

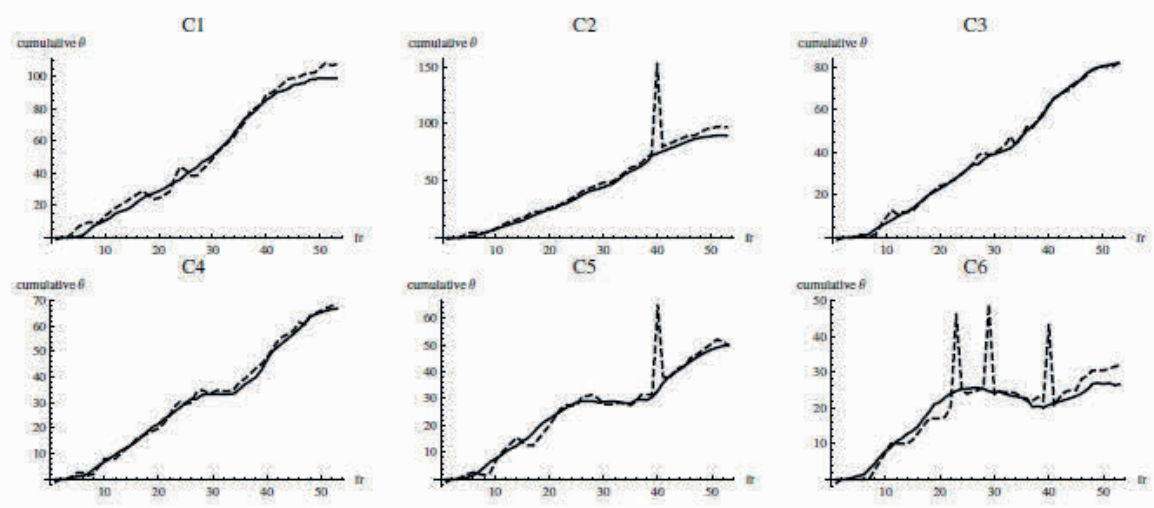

Figure 4: The cumulative angle of each vertebra through the video sequence, as marked by the user (dashed) and our algorithm (continuous).

Processing time can be shortened by reducing the video frame rate. We were able to reduce the original frame rate of 10 frames per second to 3 frames per second without any loss of accuracy. (Note that adjusting the acquisition protocol to this frame rate will lead to a significant dose reduction). If the movement between two consecutive frames is too large, the algorithm will fail to track the vertebrae correctly. An algorithm to drop consecutive frames with little movement could further decrease the computation time. The goal of this study was to develop an automated cervical vertebrae tracking algorithm that can give quantitative data on the order of movement of segments of the cervical spine. Our method is able to accurately track the skull and the cervical vertebrae throughout an entire cervical fluoroscopy video. It is less labor-intensive than manual methods, less complex and not as computationally demanding as existing methods which extract the contours of the vertebra in each frame of the video sequence. The tracking algorithm is robust and allows for large variations in the choice of the template regions. 


\section{References}

1. Van Mameren, H., et al., Cervical spine motion in the sagittal plane (I) range of motion of actually performed movements, an X-ray cinematographic study. Eur J Morphol, 1990. 28(1): p. 47-68.

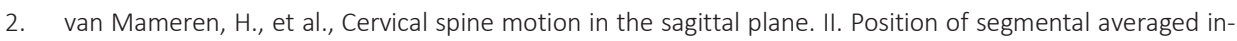
stantaneous centers of rotation--a cineradiographic study. Spine (Phila Pa 1976), 1992. 17(5): p. 467-74.

3. Howe, B., et al. Hierarchical segmentation of cervical and lumbar vertebrae using a customized generalized Hough transform and extensions to active appearance models. in 6th IEEE Southwest Symposium on Image Analysis and Interpretation, 2004. 2004.

4. Zheng, Y., M.S. Nixon, and R. Allen, Automated segmentation of lumbar vertebrae in digital videofluoroscopic images. IEEE Trans Med Imaging, 2004. 23(1): p. 45-52.

5. Roberts, M.G., T.F. Cootes, and J.E. Adams, Vertebral shape: automatic measurement with dynamically sequenced active appearance models. Med Image Comput Comput Assist Interv, 2005. 8(Pt 2): p. 733-40.

6. Chaitanya, B., Segmentation of radiographs of cervical spine using level sets. 2006, Texas Tech University.

7. Benjelloun, M. and S. Mahmoudi, X-ray image segmentation for vertebral mobility analysis. International Journal of Computer Assisted Radiology and Surgery, 2008. 2(6): p. 371-383.

8. Haber, E. and J. Modersitzki, Intensity gradient based registration and fusion of multi-modal images. Med Image Comput Comput Assist Interv, 2006. 9(Pt 2): p. 726-33. 
Chapter

\begin{abstract}
A pilot study of sequence of segmental contributions in the lower cervical spine during active extension and flexion:

healthy controls vs. cervical degenerative disc disease patients
\end{abstract}




\section{Abstract}

Study Design: A retrospective study in which, first, a definition of normal movement in the cervical spine is formulated. Second, use of this definition in differentiating between normal and abnormal movement of the cervical spine is evaluated.

Objective: To formulate a clear definition of the normal sequence of segmental contributions in the cervical spine during flexion/extension, and to evaluate the sensitivity, specificity, and reliability of sequence of segmental contributions analysis in differentiating between normal and abnormal movement of the cervical spine in healthy controls and patients with cervical degenerative disc disease (CDDD).

Summary of Background Data: To study mobility before and after cervical arthroplasty, segmental range of motion (sROM) is most commonly used. Analysis of sequence of segmental contributions during flexion and/or extension might be more suitable.

Methods: The definition was formulated by analysing flexion cinematographic recordings (FCRs) and extension cinematographic recordings (ECRs) in healthy controls. Sequences in ECRs were more consistent than in FCRs and were therefore used to define "normal" motion. Five blinded spine surgeons scored randomized graphs from ECRs in 20 healthy controls and 10 pre-operative CDDD patients, using this definition, at three time points. Sensitivity, specificity, and reliability were calculated.

Results: Reliability of scoring as normal or abnormal is high (Fleiss kappa of 0.80-0.84, range of $95 \% \mathrm{Cl}$ : 0.66- 0.98$)$, sensitivity and specificity are also high, with an average sensitivity of $90 \%$ (range of $95 \% \mathrm{Cl}: 78.4-99.8 \%$ ), and an average specificity of $85 \%$ (range of $95 \% \mathrm{Cl}: 72.9-96.4 \%)$.

Conclusions: This is the first described method which can reliably differentiate between normal or abnormal movement of the cervical spine in an individual subject. This enables an observer to determine if the normal sequence is restored after arthroplasty. In contrast to the given method, sROMs are not able to distinguish between healthy controls and CDDD patients. 


\section{Introduction}

In the treatment of cervical degenerative disc disease (CDDD), cervical arthroplasty (CA) by implanting a mobile disc prosthesis is one of the options. The advantage of CA over the more commonly performed anterior cervical discectomy with fusion (ACDF), or simple discectomy, should be prevention of adjacent segment degeneration (ASDeg) through preservation of mobility in the treated segment. ASDeg is a radiological diagnosis. Clinically symptomatic adjacent segment degeneration, or adjacent segment disease (ASDis), has an incidence of $2.9 \%$ per year [1,2].

To study mobility after CA, segmental range of motion (sROM, i.e. the amount of sagittal rotation in a segment between the maximal flexion and maximal extension position of the entire cervical spine) is most commonly used. SROMs, however, suffer from large intra- and interindividual variability [3, 4].

The goal of CA should be not only to retain mobility, but to restore normal movement, which means a physiological interplay within the motion segments. This can be expressed as the sequence of segmental contributions during active flexion and/or extension of the entire cervical spine $[3,5]$. This sequence can be determined from a flexion cinematographic recording (FCR) and/or an extension cinematographic recording (ECR), and might be a more suitable parameter than sROMs to evaluate cervical movement before and after surgery. Demonstrating this sequence requires accurate determination of the positions of the skull and cervical vertebrae on all frames of this recording. This is very time consuming with existing methods [6, 7]. The authors previously described a method to do this almost automatically [8].

The aim of the current study was fourfold, to 1) create a clear definition of the normal sequence of segmental contributions in the lower cervical spine during flexion and extension of the entire cervical spine in healthy controls, 2) to determine if preoperative patients with single level CDDD in the lower cervical spine have an abnormal sequence, 3) to determine reproducibility, sensitivity and specificity of this sequence in distinguishing between healthy controls and CDDD patients. Finally, 4) we aim to compare sequence of segmental contributions analysis to the use of sROMs in distinguishing between healthy controls and CDDD patients.

\section{Materials and methods}

\section{Sample size calculation}

Based on historic data a normal sequence of segmental contributions was expected in at least $80 \%$ of the healthy controls. We assumed this normal sequence would be present in $25 \%$ of the CDDD patients. With a two-sided significance of 0.05 and a power of 0.8 , the necessary number of participants per group is ten. 


\section{Participants - healthy controls}

Two groups (HC1-10 and HC11-20), each consisting of ten healthy controls, between 18 and 55 years of age, with a Neck Disability Index (NDI) score lower than 4 were included. Exclusion criteria were pregnancy, a history of cervical spine complaints as indicated by a previous diagnosis or during history taking, inflammatory spinal disease, and previous surgery/radiotherapy/malignancy in the cervical spine region. Findings in HC1-10 were validated against findings in HC11-20. Participant characteristics are described in Table 1.

Table 1: subject characteristics of healthy controls and cervical degenerative disc disease (CDDD) patients. Neck disability index (NDI) score is reported in points out of the maximum score of 50 points. There was a significant difference in sex $(P<0.0001)$, NDI score $(P<0.0001)$, and age $(P<0.05)$ between the groups.

\begin{tabular}{lll}
\hline & Healthy controls $(n=20)$ & CDDD patients $(n=10)$ \\
\hline Female, $n(\%)$ & $10(50 \%)$ & $9(90 \%)$ \\
NDI score, mean (SD) & $0.4(0.9)$ & $21.1(8.64)$ \\
Age in years, mean (SD) & $23.45(2.65)$ & $39.7(7.28)$ \\
\hline
\end{tabular}

\section{Participants - CDDD patients}

The ten most recent patients from a randomised controlled trial comparing CA versus simple discectomy were included (NCT00868335) [9]. Patients between 18 and 55 years of age, with single level CDDD at C5-C6 or C6-C7, and symptoms refractory to at least 8 weeks of non-surgical treatment were included in this trial. Exclusion criteria were pregnancy, inflammatory spinal disease, previous surgery/radiotherapy/malignancy in the cervical spine region, and $<2^{\circ}$ of active ROM at the target level. Preoperative FCRs/ECRs of the entire cervical spine were used.

Written informed consent was acquired from all healthy controls, as well as CDDD patients. Medical ethical committee approval was acquired (NCT00868335, NL14240.068.06 / METC-06-1-098). Participant characteristics are described in Table 1.

\section{Image acquisition}

The acquisition method of FCRs/ECRs was described in detail previously [8, 9]. In summary, a participant is placed on a bench which is placed in front of a digital X-ray detector (Toshiba Infinix VC-i, Toshiba Medical Systems Corporation, Japan). For FCRs, a lateral fluoroscopy is then acquired while the participant is performing a 'bending' maximum extension to maximum flexion movement (or vice versa for ECRs) of the head and entire cervical spine, capturing 10 frames per second. Between the flexion and extension recording the participant is encouraged to assume a neutral position and relax the cervical musculature. These recordings were analysed using image recognition software described previously [8]. This software uses normalised gradient field analysis to determine the position of the skull and vertebrae throughout all frames of a fluoroscopic recording, by comparing each frame with the median frame, based on a best-fit principle. For 
healthy controls, FCRs/ECRs were acquired at two time points, two weeks apart. For CDDD patients one recording was obtained, this was made at a maximum of two days preoperatively. An example ECR is added as Supplemental Video File 1 (available in the original publication).

\section{Definition of normal sequence of segmental contributions in the lower cervical spine}

Graphs from each of the ten healthy controls in HC1-10 and HC11-20, at both time points were collected. For all healthy controls at each time point a graph was exported depicting sagittal rotation in each of the three lower cervical motion segments (C4-C5, C5-C6, and C6-C7), set against cumulative sagittal rotation in block C4-C7 (i.e. the cumulated amount of flexion or extension). This allows the user to establish the sequence in which each of the segments contributes to flexion or extension.

Based on historic data, the sequence that appeared most promising in flexion was a biphasic pattern in block $\mathrm{C} 4-\mathrm{C} 7$, the first phase starting with a contribution in $\mathrm{C} 6-\mathrm{C} 7$, followed by $\mathrm{C} 5-\mathrm{C} 6$ and then $\mathrm{C} 4-\mathrm{C} 5[3,5]$. The second phase showed the reverse order of the first phase (i.e. cranial to caudal). The contribution of C4-C5 consists of one central peak, or two separate ones. This sequence was therefore the primary focus of a possible definition. In extension, a similar biphasic pattern was described, with the difference that the sequence in the first phase was variable. The sequence in the second phase however, was cranial to caudal in the majority of healthy controls $[3,5]$. This was a secondary focus of a possible definition.

For both flexion and extension, the first author analysed ten graphs from HC1-10 per time point to identify consistent patterns in the sequence of segmental contributions, guided by the descriptive historic data in healthy controls [5]. Based on the most consistent pattern a sequence definition was made, which was then tested against the graphs of HC1120 , to determine if the defined sequence was consistent across these two groups. For reasons explained later in the Results section a decision was made to base the sequence definition of "normal" movement on the extension data, consequently, all subsequent analyses to determine the reproducibility, sensitivity and specificity of this definition were based on extension data only.

\section{Sequence in patients}

Additionally, graphs from the ten CDDD patients at the preoperative time point were analysed. These ten graphs were checked by the first author for the presence or absence of the sequence identified in the healthy controls, based on the sequence definition from the healthy controls. 


\section{Determination of reproducibility, sensitivity and specificity}

Reproducibility, sensitivity and specificity of distinguishing healthy controls from CDDD patients were determined, based on the sequence definition. Ten randomly chosen extension graphs from HC1-10 (HC1-extT1, HC2-extT2, HC3-extT1, HC4-extT1, HC5-extT1, HC6extT2, HC7-extT1, HC8-extT2, HC9-extT2, HC10-extT1), as well as all ten extension graphs from CDDD patients were scored by five blinded independent spinal surgeons, at two time points ( 11 and $\mathrm{t} 2$ ), at least one week apart. They were asked to score the twenty graphs, presented in a random order, as being 'normal' or 'not normal', based on the sequence definition. At a third time point ( $\mathrm{t} 3$ ) they were asked to score twenty graphs again, the same ten graphs from CDDD patients, and ten random extension graphs from HC11-20 (HC11-extT1, HC12-extT2, HC13-extT1, HC14-extT2, HC15-extT1, HC16-extT1, HC17extT2, HC18-extT2, HC19-extT1, HC20-extT2) instead of from HC1-10.

\section{Calculation of SROMs}

SROMs were calculated for segments $\mathrm{C} 4-\mathrm{C} 5, \mathrm{C} 5-\mathrm{C} 6$, and $\mathrm{C} 6-\mathrm{C} 7$ by summating all the sagittal rotation values of a motion segment between consecutive frames. This was done for all CDDD patients and healthy controls.

\section{Statistics}

To determine reproducibility Fleiss' kappa values were calculated for each time point as a measure of interobserver agreement, kappa values were calculated for each observer as a measure of intra-observer agreement.

Sensitivity and specificity were calculated, based on the normal sequence definition. Sensitivity and specificity were calculated by conventional methods, i.e. the percentage of CDDD patients' scorings with an abnormal test results, and healthy controls scorings with a normal test result. Wolfram Mathematica (Wolfram Research, Inc., Version 9.0, Champaign, IL) was used for all statistical calculations.

Differences in SROM between healthy controls and CDDD patients were analysed using an independent samples t-Test, after testing for normality. Statistical significance was defined as $\mathrm{P}<0.05$.

\section{Results}

In FCRs, the earlier described biphasic sequence within block C4-C7 was present in a minority of the graphs of HC1-10 and HC11-20, in six of twenty analyses in HC1-10 and in five of twenty analyses in HC11-20 (Appendix 1). The most consistent finding in FCRs was the presence of the first phase of flexion, with a sequence of segmental contributions from caudal to cranial. In $\mathrm{HC1}-10$ this first phase was present at both time points in five 
of ten healthy controls, and at one time point in four of ten healthy controls. In HC11-20 it was present at both time points in six of ten healthy controls, and at one time point in three of ten healthy controls.

A much more consistent sequence of segmental contributions was identified in the ECRs of HC1-10, in the last phase of the extension movement. It consisted of a peak in rotation in C4-C5, followed by a peak in C5-C6, and finally in C6-C7 (Figure 1).

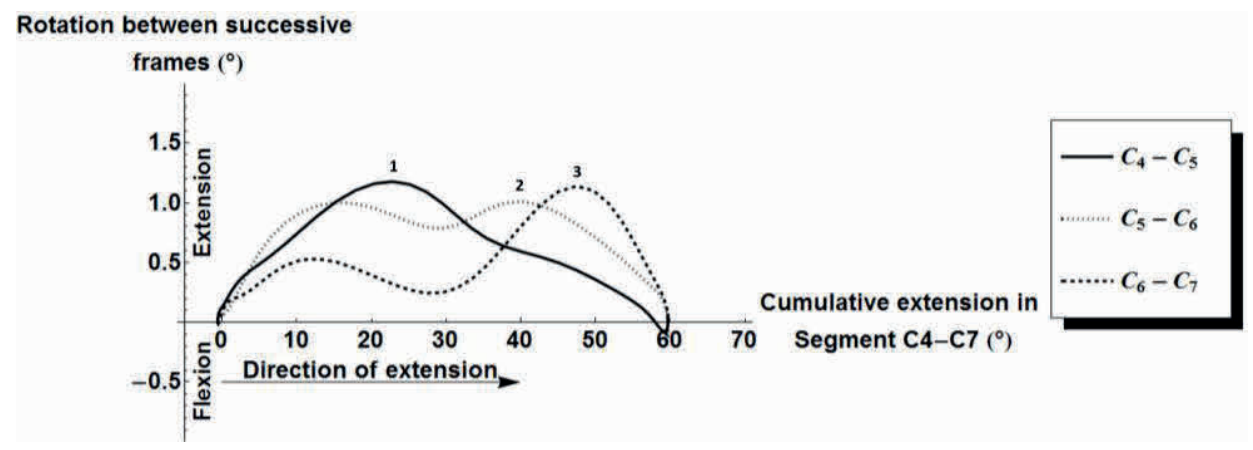

Figure 1: graph depicting sagittal rotation in segments in the lower cervical spine (block C4-C7) during extension of the entire cervical spine, in a healthy control. On the y-axis sagittal rotation between successive frames is shown. On the $\mathrm{x}$-axis cumulative extension in block C4-C7 is shown. Peaks in the graphs depict maximum contributions of a segment in that phase of extension of the entire cervical spine. From left to right a peak in contribution of C6-C7 is followed by a peak in contribution of C5-C6, and then C4-C5 (1). In the following phase, from about 25 up to 60 degrees of extension between C4 and C7 the peak of C4-C5 (1) is followed by a peak in C5-C6 (2), and then C6-C7 again (3). This last phase (1-2-3) is common in extension in healthy controls. Each series of values undergoes smoothing by means of a low-pass Gaussian digital filter (Oth order, $\sigma=2.9$ ).

Only the sequence of the peaks was important, not the height of the peaks in itself. Very small peaks with a rotation lower than $0.3^{\circ}$, which are deemed to fall within measurement error (see Appendix 2), were not taken into consideration. In many cases there was a peak in C4-C5 at the end of extension, which was much smaller than the peak at (1), which was considered normal. This sequence was present in HC1-10 in 80 percent $(8 / 10)$ at $\mathrm{T} 1$, and 90 percent $(9 / 10)$ at $\mathrm{T} 2$.

Consistency of results was much higher for ECRs then FCRs (see Appendix 3). Based on the findings in the ECRs of HC1-10 a definition was written to describe the normal sequence of segmental contributions in extension, to enable objective scoring by multiple observers (Appendix 4). Using this definition, HC11-20 was scored by the first author. Results in this group were similar, with the sequence defined as normal present in 90 percent $(9 / 10)$ at $T 1$, and 80 percent $(8 / 10)$ at $T 2$. The forty graphs from the extension analyses in HC1-10 and HC11-20 are added as Appendix 3.

To answer the second aim of the study, the CDDD patients were also studied by the first author. The sequence defined as normal in the previous paragraph was only present in 10 percent (1/10) of the ECRs. All ten graphs are depicted in Appendix 5. 
Sensitivity in differentiating between normal and not-normal, based on the scores of the five independent spine surgeons, was $88-92 \%$ (range of $95 \% \mathrm{Cl}: 78.4-99.8 \%$ ), specificity was $84-86 \%$ (range of $95 \% \mathrm{Cl}: 72.9-96.4 \%$ ) (Table 2). Fleiss' kappa was 0.80-0.84 (range of $95 \% \mathrm{Cl}$ : 0.66- 0.98). Average Kappa for the five observers was 0.850 (range of $95 \% \mathrm{Cl}: 0.663-1.000)$.

Average sROMs (SD) for flexion and extension studies combined were calculated for HC1-10, HC11-20 and CDDD patients (Appendix 6). Group average sROM for the CDDD patients was significantly lower than in the healthy controls for C5-C6 and C6-C7 (Table 3). For C4-C5 there was no significant difference $(P=0.73)$.

Table 2: test characteristics of five spine surgeons scoring cervical spine movement as being normal or abnormal using the definition that was written, at three time points ( $t 1, t 2$, and $t 3$ ). At each time point twenty graphs were scored (10 healthy controls and 10 cervical degenerative disc disease patients).

\begin{tabular}{llll}
\hline & t1 & t2 & t3 \\
\hline Fleiss' kappa $(95 \% \mathrm{Cl})$ & $0.84(0.70-0.98)$ & $0.80(0.66-0.94)$ & $0.80(0.66-0.94)$ \\
Sensitivity $(95 \% \mathrm{Cl})$ & $92 \%(84.2-99.8 \%)$ & $88 \%(78.4-97.6 \%)$ & $90 \%(81.2-98.8 \%)$ \\
Specificity $(95 \% \mathrm{Cl})$ & $86 \%(75.6-96.4 \%)$ & $84 \%(72.9-95.1 \%)$ & $86 \%(75.6-96.4 \%)$ \\
Kappa (95\% Cl) & & \\
Observer 1 & $0.798(0.663-1,000)$ & Not applicable \\
Observer 2 & $0.900(0.803-1,000)$ & \\
Observer 3 & $0.900(0.803-1,000)$ & \\
Observer 4 & $0.802(0.672-1,000)$ & \\
Observer 5 & $0.900(0.803-1,000)$ & \\
\hline
\end{tabular}

Table 3: Average segmental range of motion (SROM) and their respective standard deviation (SD) for motion segments C4-C5, C5-C6, and C6-C7 for HC1-10, HC11-20, and CDDD group, as well as for reported populations of healthy controls. Average sROM for the CDDD patients is significantly lower than in healthy controls (HC1-10 and HC11-20 combined) for C5-C6 ( $=0.0046)$, and for C6-C7 ( $=0.015)$. For C4-C5 there was no significant difference $(P=0.73)$.

\begin{tabular}{|c|c|c|c|}
\hline \multirow[t]{2}{*}{ This study } & \multicolumn{3}{|c|}{ Mean sROM $\left({ }^{\circ}, \mathrm{SD}\right)$} \\
\hline & $\mathrm{C} 4-\mathrm{C5}$ & $\mathrm{C} 5-\mathrm{C} 6$ & $\mathrm{C6}-\mathrm{C} 7$ \\
\hline HC1-10 & $18.4(3.4)$ & $22.0(1.4)$ & $19.1(2.4)$ \\
\hline HC11-20 & $15.6(2.2)$ & $17.5(3.7)$ & $16.7(5.4)$ \\
\hline CDDD & $17.4(3.4)$ & $14.7(5.1)^{* *}$ & $13.6(3.7)^{*}$ \\
\hline \multirow{2}{*}{\multicolumn{4}{|c|}{$\begin{array}{l}{ }^{* *} P<0.005 \\
{ }^{*} P<0.05\end{array}$}} \\
\hline & & & \\
\hline \multirow[t]{2}{*}{ Literature } & \multicolumn{3}{|c|}{ Mean sROM $\left({ }^{\circ}, \mathrm{SD}\right)$} \\
\hline & $\mathrm{C} 4-\mathrm{C} 5$ & $\mathrm{C} 5-\mathrm{C} 6$ & $\mathrm{C6}-\mathrm{C} 7$ \\
\hline Aho et al. & $22(4)$ & $28(4)$ & $15(4)$ \\
\hline Bhalla et al. & $23(1)$ & $19(1)$ & $18(3)$ \\
\hline Lind et al. & $16(6)$ & $15(8)$ & $11(7)$ \\
\hline Dvorak et al. & $19(4)$ & $20(4)$ & $19(4)$ \\
\hline From: Bogdul & & & \\
\hline
\end{tabular}




\section{Discussion}

This study shows that the sequence of segmental contributions in the lower cervical spine during the second half of extension of the entire cervical spine and head was uniform in healthy controls. The sequence of segmental contributions was $\mathrm{C} 4-\mathrm{C} 5$ followed by $\mathrm{C} 5-\mathrm{C} 6$, and then C6-C7 (Appendix 4). This sequence was present in $80-90 \%$ of healthy controls. In the group of single level CDDD patients the sequence as described in the sequence definition, was present in only one CDDD patient (10\%). Using a clear definition of a normal sequence of segmental contributions, reliability of scoring as normal or abnormal was high, as was proven by a high Fleiss Kappa of 0.80-0.84. Sensitivity and specificity were also high, with an average sensitivity of $90 \%$, and an average specificity of $85 \%$. This method is the first that can reliably differentiate between normal or abnormal movement of the cervical spine in an individual. These characteristics make it a useful tool in the follow-up of cervical arthroplasty, as it enables an observer to reliably determine if the normal sequence is restored.

Results for sequence of segmental contributions in FCRs were less consistent than was expected based on the historic data. A reason for the difference in the findings in FCRs could be the different investigator that was instructing the healthy controls (TB in this study, versus HvM in the historic control group). Also, participants were sitting on a chair in the historic control group, and on a little bench in the current study, which could lead to a difference in sitting position. In contrast, in ECRs the results were very consistent. This suggests that the sequence of segmental contributions is less dependent on these factors during the extension movement.

The most commonly used tool to evaluate segmental motion after arthroplasty is to determine the sROM [10-14]. However, the high intra- and interindividual variability severely limits its use in individual patients [7, 15-17]. This high interindividual variability is also present within the healthy controls in this study, with differences up to $17^{\circ}$ between healthy controls in corresponding motion segments. SROMs in all healthy controls fall within the normal ranges (defined as average +/- 2SD) that have been previously reported (Appendix 6) $[4,7,15,18,19]$. Interestingly, sROMs for all CDDD patients fall within normal ranges as well (Appendix 6), with the possible exception of one CDDD patient (CDDD4) in which a sROM of $3.0^{\circ}$ was present in C5-C6 (which was the symptomatic level). This SROM value would fall within normal range of only one of the reported groups (Lind et al.), but none of the others $[3,15]$. Using sROMs an observer is therefore not able to distinguish between healthy controls and CDDD patients in individuals. This is in contrast with analysis of the sequence of segmental contributions. On a group level sROMs were significantly lower for the CDDD patients in C5-C6 and C6-C7. This might be a result of CDDD, it could also be due to the lower average age in the healthy controls.

The reason why only the most recent ten CDDD patients of the RCT were used in this analysis instead of all 24 of them was twofold. First, based on the sample size calculation for the current study ten CDDD patients were needed. Second, due to less consistent 
results during analysis of FCRs in the healthy controls compared to historic data we hypothesized that the instruction of the participants should be stricter than has been done in earlier patients in our RCT, to avoid 'nodding' flexion, instead of 'bending' flexion. The more strict instructions were given in all of these ten most recently included CDDD patients.

In the CDDD patients there was a high female predominance (90\%). This was by chance, as $63 \%$ of the included population in the RCT is female. Female predominance in CDDD is in line with most surgical literature [11, 14, 20-22]. An influence of gender predominance on the sequence of segmental contributions results is not likely since male and female healthy controls were evenly distributed and showed similar results. The average age in de CDDD patients groups was higher (Table 1). Although an influence of age on the sequence of segmental contributions cannot be excluded, it is not likely. Because in the four youngest patients (all between 26 and 36 years old) only one had a normal sequence (CDDD6-ext), while the five oldest healthy controls in group HC11-20 (all between 26 and 29 years old) had a normal sequence in 8 of 10 ECRs.

Our definition of normal sequence was based only on ECRs in HC1-10, while HC11-20 was scored using this definition with similar results. Results for the blinded observers were also similar for HC1-10 (graphs used at t1 and t2) and HC11-20 (graphs used at t3). When the original graphs in the historic control group were checked for this sequence, it was also present in nine out of ten healthy controls at all three time points that were recorded, and in the tenth healthy control in two out of three time points [5]. The pattern is therefore present in three separate groups of healthy controls, further proving the consistency of the sequence described in ECRs.

The origin of this sequence of segmental contributions is not known. Most likely it results from interplay between muscle activity and gravity, as the latter would be the most efficient way to accomplish later phases in extension. The disturbed sequence of segmental contributions could be analogous to previously described altered patterns in muscle recruitment in patients with chronic neck pain [23]. It could be a way of the body to avoid pain due to radicular compression by narrowing of the neuroforamen during the extension movement. A correlation between the level of disc degeneration and a specific sequence could not be identified as most (9/10) of the CDDD patients in the group that were analysed had CDDD at C5-C6.

This method of analysing movement in the lower cervical spine will be used in our currently ongoing RCT to evaluate if the findings in the currently described group of CDDD patients can be reproduced in a larger sample size of preoperative patients, and if so, if a normal sequence of segmental contributions returns to patients with CDDD after arthroplasty, which is the premise on which prevention of ASDis is based. Additionally, future plans include applying this method of analysis to a group of older healthy controls, to exclude the possibility of age being a confounding factor. 


\section{Key points:}

- Analysis of the sequence of segmental contributions is the first described method, which can reliably differentiate between normal or abnormal movement of the cervical spine in an individual subject.

- A clear definition of a normal sequence of segmental contributions in the lower cervical spine in ECRs is provided.

- Reliability of scoring as normal or abnormal sequence in ECRs is high (Fleiss kappa of $0.80-0.84$, range of $95 \% \mathrm{Cl} 0.66-0.98)$. Sensitivity and specificity are also high, resp. $90 \%$ (range of $95 \% \mathrm{Cl} 78.4-99.8 \%$ ), and $85 \%$ (range of $95 \% \mathrm{Cl} 72.9-96.4 \%$ ).

- This method is a useful tool in the follow-up of cervical arthroplasty, as it enables an observer to reliably determine whether the normal sequence of segmental contributions is restored.

- The often used sROMs are not able to distinguish between healthy controls and CDDD patients. 


\section{References}

1. Hilibrand AS, Carlson GD, Palumbo MA, et al. Radiculopathy and myelopathy at segments adjacent to the site of a previous anterior cervical arthrodesis. J Bone Joint Surg Am 1999;81:519-28.

2. Seo M, Choi D. Adjacent segment disease after fusion for cervical spondylosis; myth or reality? $\mathrm{Br} J \mathrm{Neu}$ rosurg 2008;22:195-9.

3. Bogduk N, Mercer S. Biomechanics of the cervical spine. I: Normal kinematics. Clin Biomech (Bristol, Avon) 2000;15:633-48.

4. Van Mameren H, Drukker J, Sanches H, et al. Cervical spine motion in the sagittal plane (I) range of motion of actually performed movements, an X-ray cinematographic study. Eur J Morphol 1990;28:47-68.

5. Van Mameren H. Motion patterns in the cervical spine: Maastricht University, 1988.

6. Penning L. Normal movements of the cervical spine. AJR Am J Roentgenol 1978;130:317-26.

7. Dvorak J, Froehlich D, Penning L, et al. Functional radiographic diagnosis of the cervical spine: flexion/extension. Spine (Phila Pa 1976) 1988;13:748-55.

8. Reinartz R, Platel B, Boselie T, et al. Cervical vertebrae tracking in video-fluoroscopy using the normalized gradient field. Med Image Comput Comput Assist Interv 2009;12:524-31.

9. Boselie TF, van Mameren $\mathrm{H}$, de Bie RA, et al. Cervical spine kinematics after anterior cervical discectomy with or without implantation of a mobile cervical disc prosthesis; an RCT. BMC Musculoskelet Disord 2015;16:34.

10. Sasso RC, Best NM. Cervical kinematics after fusion and bryan disc arthroplasty. Journal of spinal disorders \& techniques 2008;21:22.

11. Coric D, Nunley PD, Guyer RD, et al. Prospective, randomized, multicenter study of cervical arthroplasty: 269 patients from the Kineflex $\mid C$ artificial disc investigational device exemption study with a minimum 2year follow-up. J Neurosurg Spine 2011.

12. Kelly MP, Mok JM, Frisch RF, et al. Adjacent Segment Motion Following Anterior Cervical Discectomy and Fusion versus ProDisc(R)-C Cervical Total Disk Arthroplasty: Analysis from a Randomized, Controlled Trial. Spine (Phila Pa 1976) 2011.

13. Park JJ, Quirno M, Cunningham MR, et al. Analysis of segmental cervical spine vertebral motion after prodisc-C cervical disc replacement. Spine 2010;35:E289.

14. Mummaneni PV, Burkus JK, Haid RW, et al. Clinical and radiographic analysis of cervical disc arthroplasty compared with allograft fusion: a randomized controlled clinical trial. J Neurosurg Spine 2007;6:198-209.

15. Lind B, Sihlbom H, Nordwall A, et al. Normal range of motion of the cervical spine. Arch Phys Med Rehabil 1989;70:692-5.

16. Frobin $W$, Leivseth $G$, Biggemann $M$, et al. Sagittal plane segmental motion of the cervical spine. A new precision measurement protocol and normal motion data of healthy adults. Clin Biomech (Bristol, Avon) 2002;17:21-31.

17. Wu SK, Kuo LC, Lan HC, et al. The quantitative measurements of the intervertebral angulation and translation during cervical flexion and extension. Eur Spine J 2007;16:1435-44.

18. Bhalla SK, Simmons EH. Normal ranges of intervertebral-joint motion of the cervical spine. Can J Surg 1969;12:181-7.

19. Aho A, Vartiainen O, Salo O. Segmentary antero-posterior mobility of the cervical spine. Ann Med Intern Fenn 1955;44:287-99.

20. Heller JG, Sasso RC, Papadopoulos SM, et al. Comparison of BRYAN cervical disc arthroplasty with anterior cervical decompression and fusion: clinical and radiographic results of a randomized, controlled, clinical trial. Spine (Phila Pa 1976) 2009;34:101-7.

21. Riina J, Patel A, Dietz JW, et al. Comparison of single-level cervical fusion and a metal-on-metal cervical disc replacement device. Am J Orthop 2008;37:E71-7. 
22. Murrey D, Janssen M, Delamarter R, et al. Results of the prospective, randomized, controlled multicenter Food and Drug Administration investigational device exemption study of the ProDisc-C total disc replacement versus anterior discectomy and fusion for the treatment of 1-level symptomatic cervical disc disease. Spine J 2009;9:275-86.

23. Tsang SM, Szeto GP, Lee RY. Altered spinal kinematics and muscle recruitment pattern of the cervical and thoracic spine in people with chronic neck pain during functional task. J Electromyogr Kinesiol 2014;24:104-13. 


\section{Appendix 1: Flexion graphs - healthy controls $\mathrm{HC} 1-10$ at T1 \\ Appendix}

Graphs depicting sagittal rotation in segments in the lower cervical spine (block C4-C7) during flexion of the entire cervical spine, for all healthy controls, at both time points. Peaks in the graphs depict maximum contributions of a segment in that phase of flexion of the entire cervical spine. In these graphs flexion is depicted from right to left. Graphs for $\mathrm{HC} 1-10$ are presented in page 1 and 2 in the same order. Graphs for $\mathrm{HC} 11-20$ are presented in page 3 and 4 , also in the same order. Based on historic data the sequence that appeared most promising was a biphasic pattern in block $\mathrm{C} 4-\mathrm{C} 7$, the first phase starting with $\mathrm{C} 6-\mathrm{C} 7$, followed by $\mathrm{C} 5-\mathrm{C} 6$ and then $\mathrm{C} 4-\mathrm{C} 5$. The second phase in the reverse order of the first phase (cranial to caudal). The contribution of C4-C5 consisting of one central peak, or two.

This biphasic sequence was only present in six of twenty analyses in (HC1-flexT1, HC4-flexT1, HC8-flexT1, HC3flexT2, HC4-flexT2, HC8-flexT2). The first phase was present at both time points in five of ten controls (HC3, HC4, $\mathrm{HC} 7, \mathrm{HC} 8, \mathrm{HC} 9)$, and at one time point in four of ten controls ( $\mathrm{HC} 1, \mathrm{HC} 2, \mathrm{HC} 6, \mathrm{HC} 10)$. It was not present at either time point in one control ( $\mathrm{HC5}$ ). The second phase was present less frequently, at both time points in four of ten controls ( $\mathrm{HC} 1, \mathrm{HC} 4, \mathrm{HC} 5, \mathrm{HC}$ ), at one time point in three of ten controls ( $\mathrm{HC} 2, \mathrm{HC}, \mathrm{HC} 10)$, and not present at all in one of ten controls ( $\mathrm{HC9}$ ).

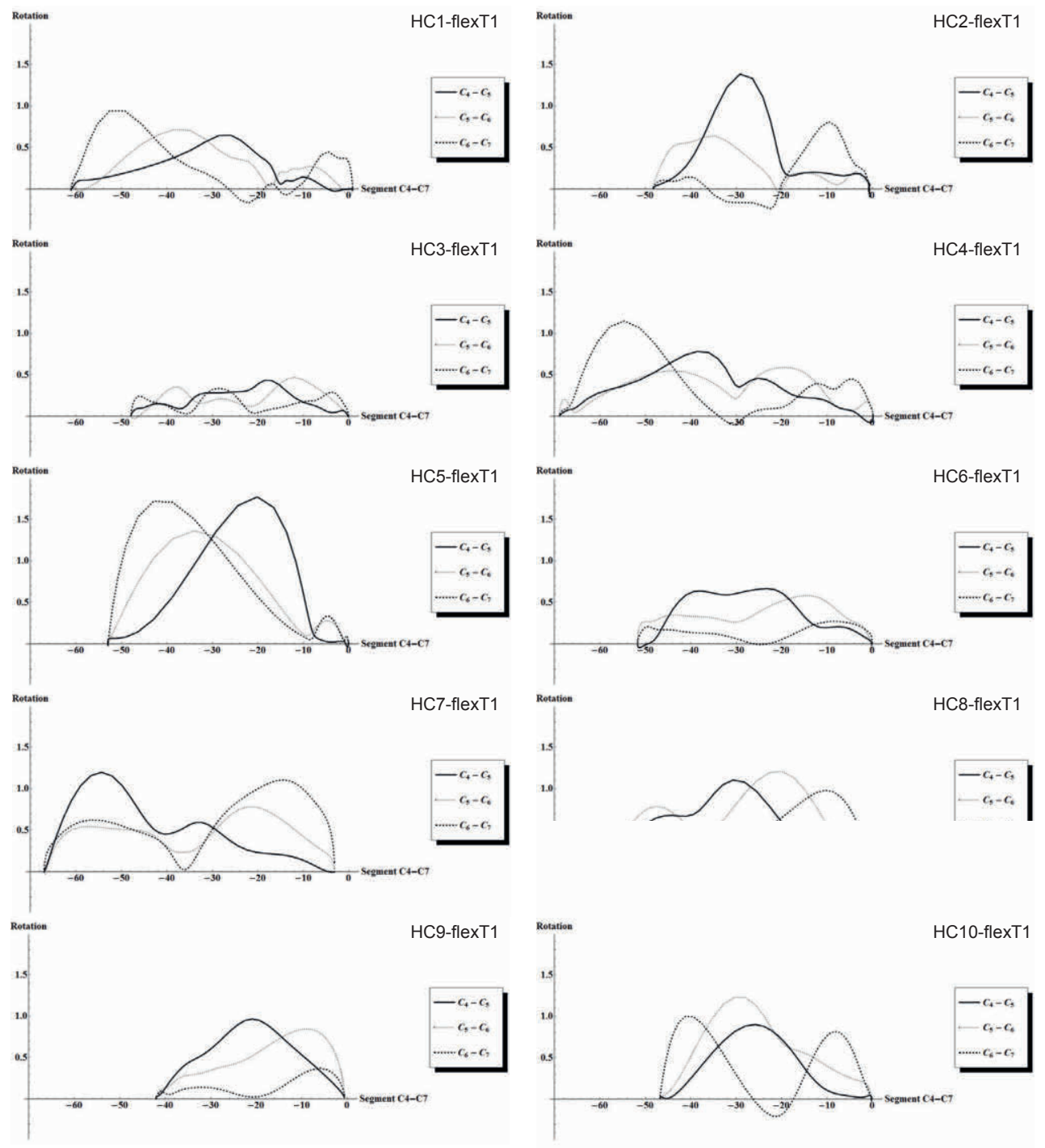


Appendix 1: Flexion graphs - healthy controls HC1-10 at T2

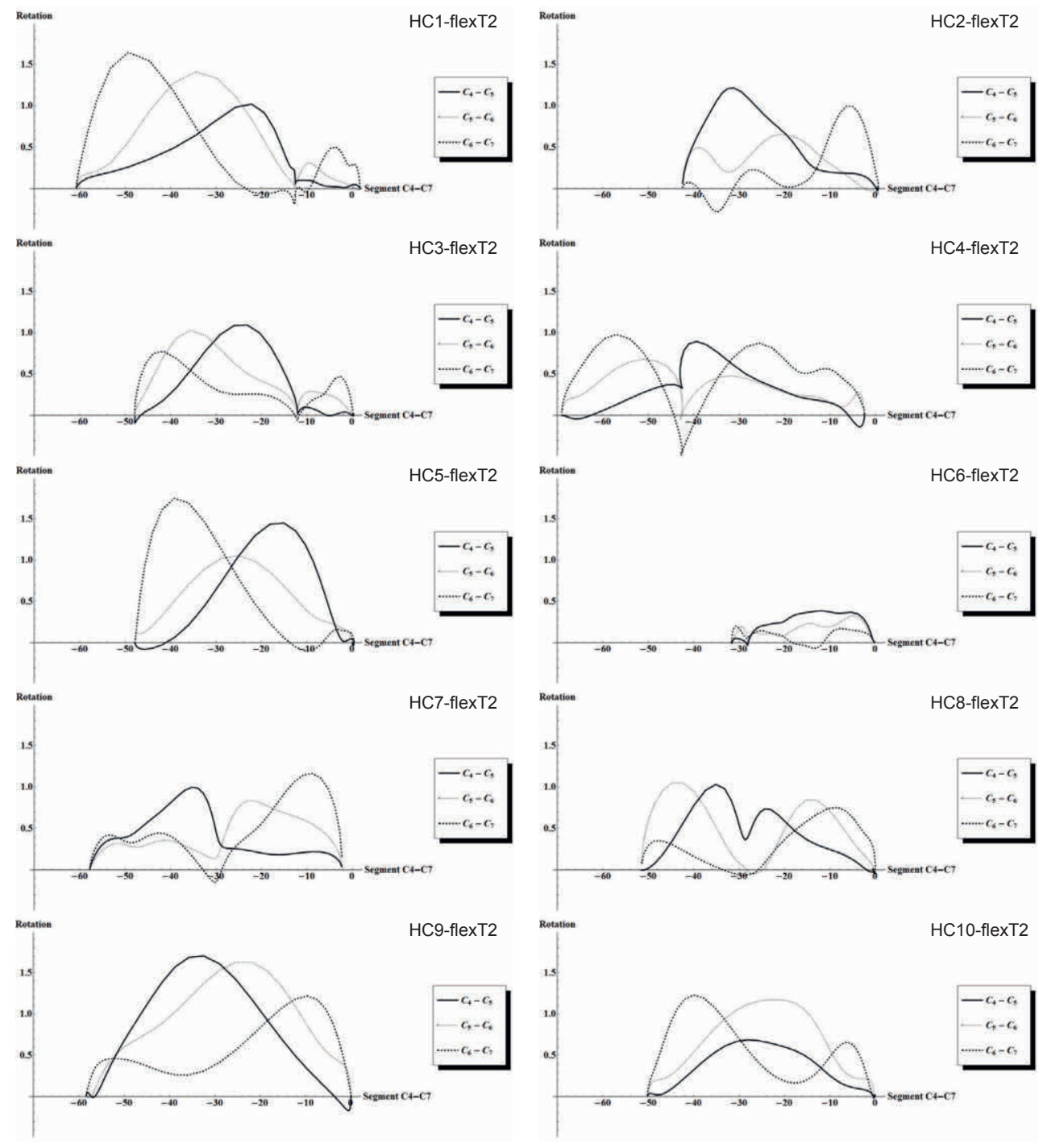




\section{Appendix 1: Flexion graphs - healthy controls $\mathrm{HC} 11-20$ at T1}

In group two the biphasic sequence was only present in five of twenty analyses in group 2 (HC19-flexT1, HC20flexT1, HC13-flexT2, HC15-flexT2, HC19-flexT2). The first phase was present at both time points in six of ten controls (HC12, HC13, HC15, HC16, HC17, HC19), at one time point in three of ten controls (HC14, HC18, HC20), and not present at all in one of ten controls ( $\mathrm{HC11}$ ). The second phase was present at both time points in one of ten controls ( $\mathrm{HC19}$ ), and at one time point in three of ten controls ( $\mathrm{HC13}, \mathrm{HC15}, \mathrm{HC} 20)$. It was not present at either time point in the remaining six controls.

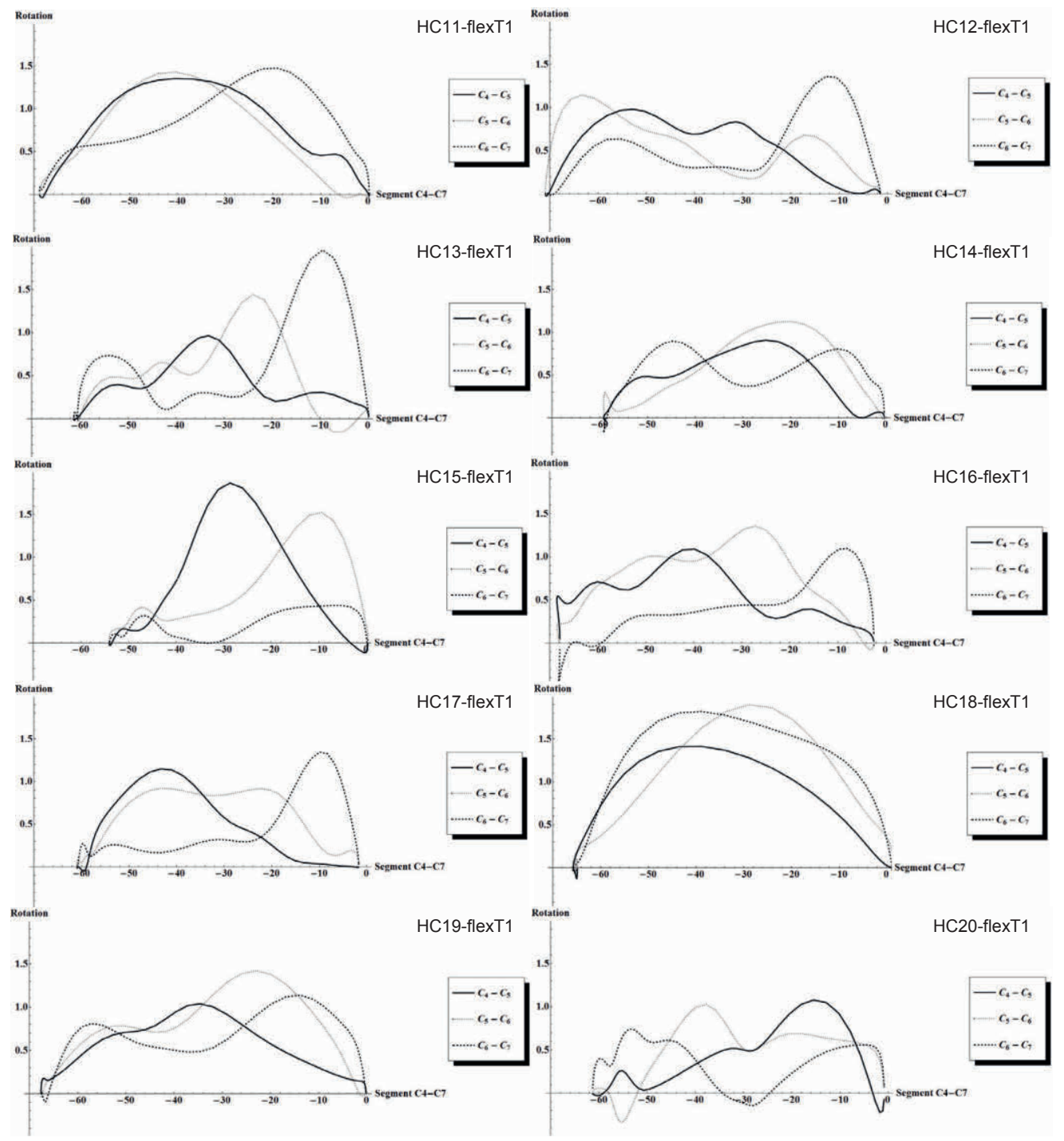


Appendix 1: Flexion graphs - healthy controls HC11-20 at T2

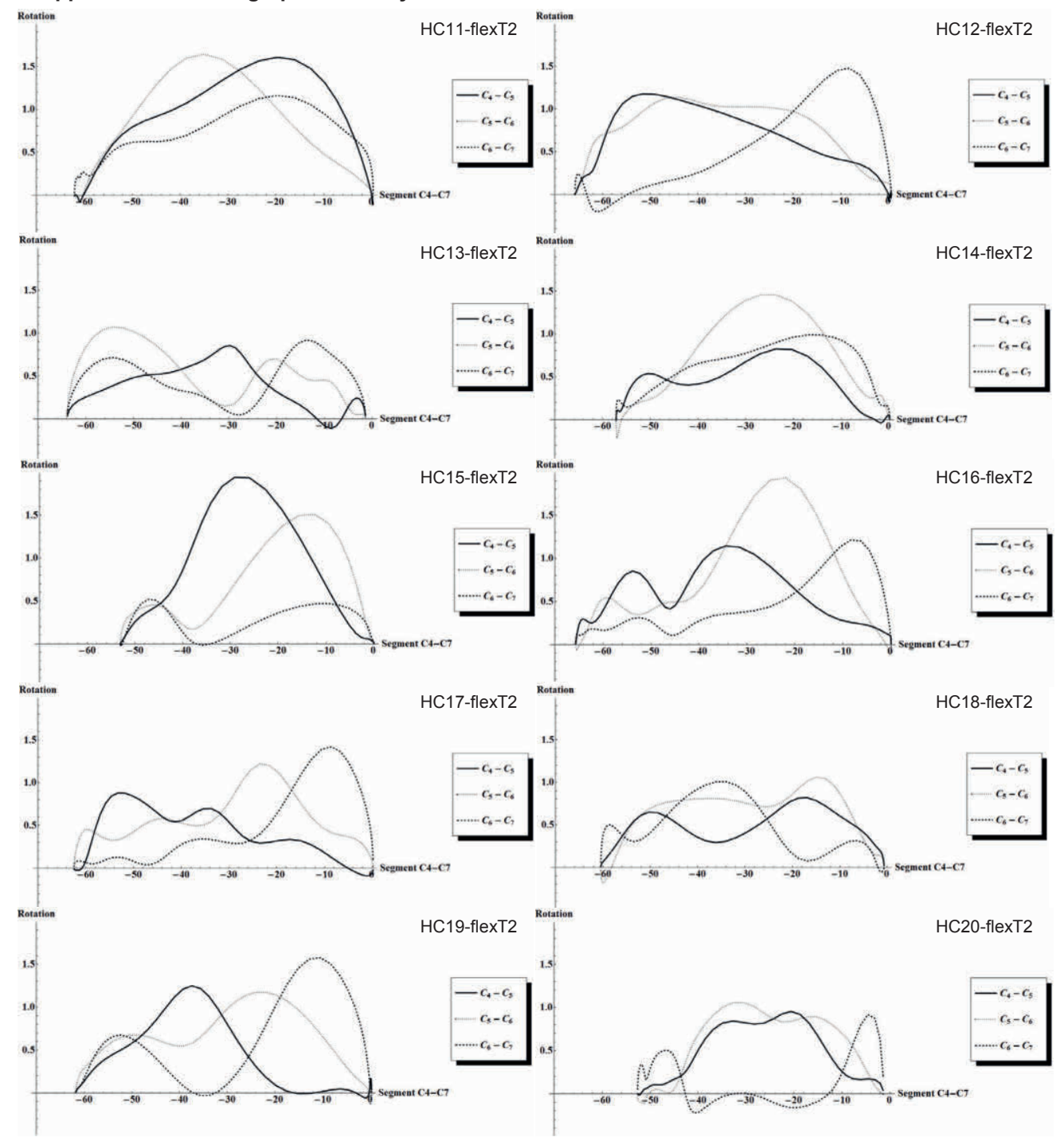




\section{Appendix 2: precision of tracing vertebral contours in the lower cervical spine}

Two recordings were each analysed five times by one author (TB), each run includes drawing of the areas of interest for the image recognition algorithm, the automated analysis by the software, and manually checking the traced contours of the lower cervical vertebrae on all frames and correcting these where needed. One recording was of good image quality (an FCR of HC1), and one recording of bad image quality (an ECR of a CDDD patient that is not described in the current publication). Graphs for rotation in C4-C5, C5-C6 and C6-C7 are depicted for all five analysis runs of HC1-flexT2 on page 2 of this Appendix. The same is done for all five analysis runs of CDDD-ext on page 3 of this Appendix.

Graphs are very similar for the recording of $\mathrm{HC} 1$. The sequence of segmental contributions is the same in all five analysis runs, at the right side a peak in C4-C5 is followed by a peak in C5-C6, and finally C6-C7.

For the recording of CDDD-ext, graphs are less similar. However, the locations of the peaks of the graphs, and therefore the sequence of segmental contributions is the same in all five analysis runs, except for very small peaks that appear, for instance near the end of the graph of C4-C5 in run 1 and 3.

Additionally, precision of using this method was calculated by calculation of intra-observer ICCs, two-way mixed, testing for absolute agreement of single measures. To quantify agreement, repeatability standard deviations $\left(\mathrm{SD}_{\mathrm{r}}\right)$ were calculated.

Intra-observer ICCs were high for all three levels in both recordings (Table S1), indicating good to excellent precision.

\begin{tabular}{|c|c|c|c|}
\hline Recording & Level & ICC & 95\% confidence interval \\
\hline CDDD-ext & C4-C5 & 0.842 & $0.776-0.897$ \\
\hline & C5-C6 & 0.894 & $0.847-0.932$ \\
\hline & C6-C7 & 0.804 & $0.716-0.873$ \\
\hline HC1-flexT2 & C4-C5 & 0.994 & $0.991-0.996$ \\
\hline & C5-C6 & 0.990 & $0.984-0.994$ \\
\hline & C6-C7 & 0.994 & $0.991-0.997$ \\
\hline
\end{tabular}

Table S1: Intraclass correlation coefficients (ICCs) with 95\% confidence intervals for each level in each recording, based on the five analysis runs by one author (TB) for each of these recordings.

Repeatability standard deviations $\left(S_{\mathrm{r}}\right)$ were calculated for each of 50 values (each value is based on the comparison of two consecutive frames of a recording), for each level in each recording (Table S2).

\begin{tabular}{|c|c|c|c|}
\hline Recording & Level & $\boldsymbol{\mu S D}_{\mathrm{r}}\left(^{\circ}\right)$ & Range \\
\hline CDDD-ext & C4-C5 & 0.091 & $0.013-0.145$ \\
\hline & C5-C6 & 0.096 & $0.011-0.195$ \\
\hline & C6-C7 & 0.080 & $0.017-0.117$ \\
\hline HC1-flexT2 & C4-C5 & 0.026 & $0.000-0.050$ \\
\hline & C5-C6 & 0.041 & $0.007-0.120$ \\
\hline & C6-C7 & 0.044 & $0.011-0.093$ \\
\hline
\end{tabular}

Table S2: Average repeatability standard deviations $\left(\mu \mathrm{SD}_{\mathrm{r}}\right.$, in degrees) for each level in the two recordings, the average is based on the 50 values that are being calculated for that level in that recording. The range of these $50 \mathrm{SD}_{\mathrm{r}}$ values is also given.

If the measurement error is defined as $\pm 2 \mu \mathrm{SD}_{\mathrm{r}}$ this amounts to $\pm 0.05^{\circ}$ to $\pm 0.2^{\circ}$. To err on the safe side, it was then decided that peaks smaller than $0.3^{\circ}$ were deemed to fall within measurement error. 

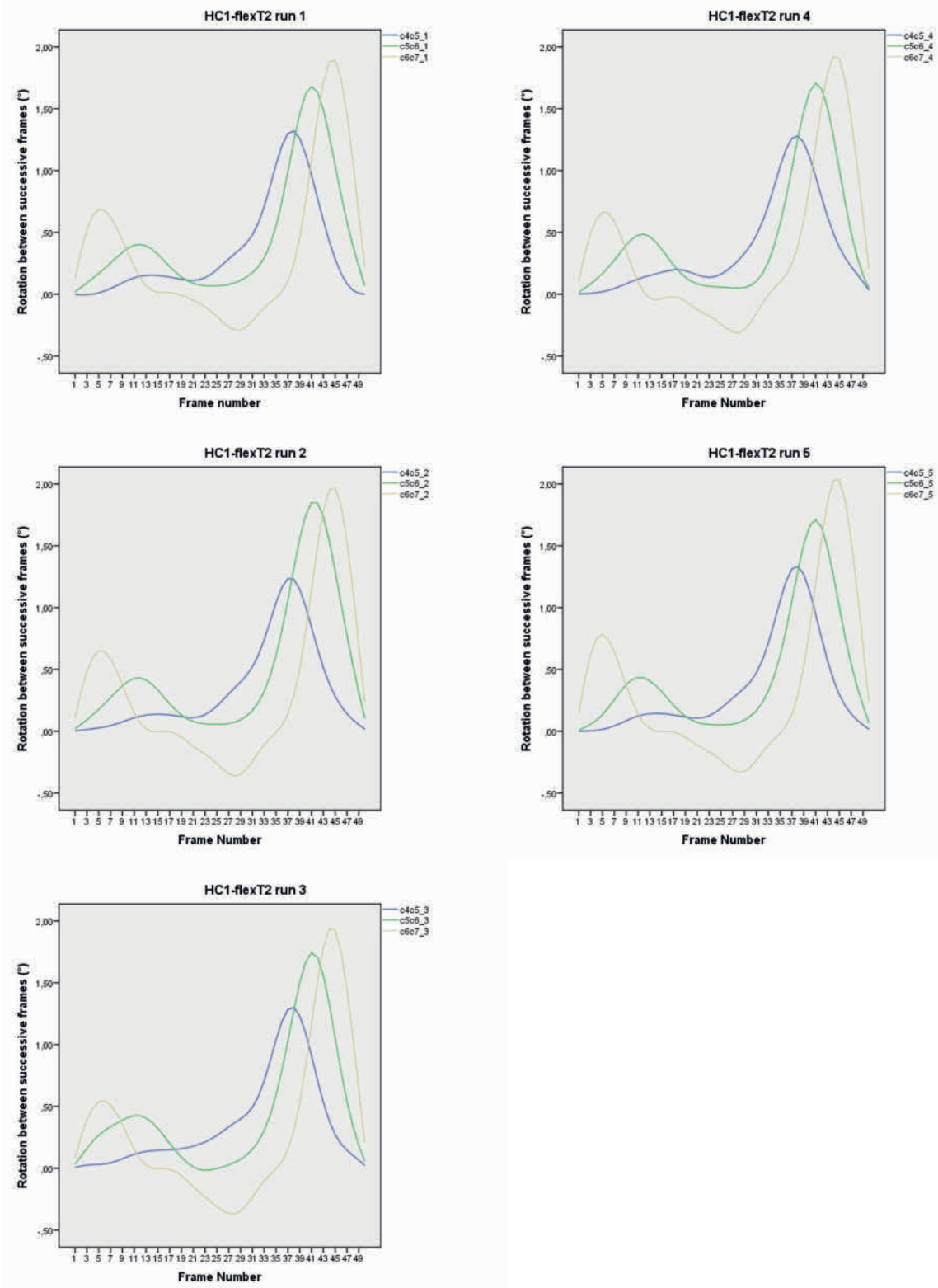

Figure S1: graphs depicting rotation (in degrees, y-axis) in the lower cervical motion segments, as a function of the frame number (x-axis) of the recording (HC1-flexT2). The five graphs each depict one of the five analysis runs that was performed to determine precision and repeatability. The location of the peaks with respect to each other, and therefore the sequence in which the motion segments contribute to the flexion movement, is the same in all five graphs. 

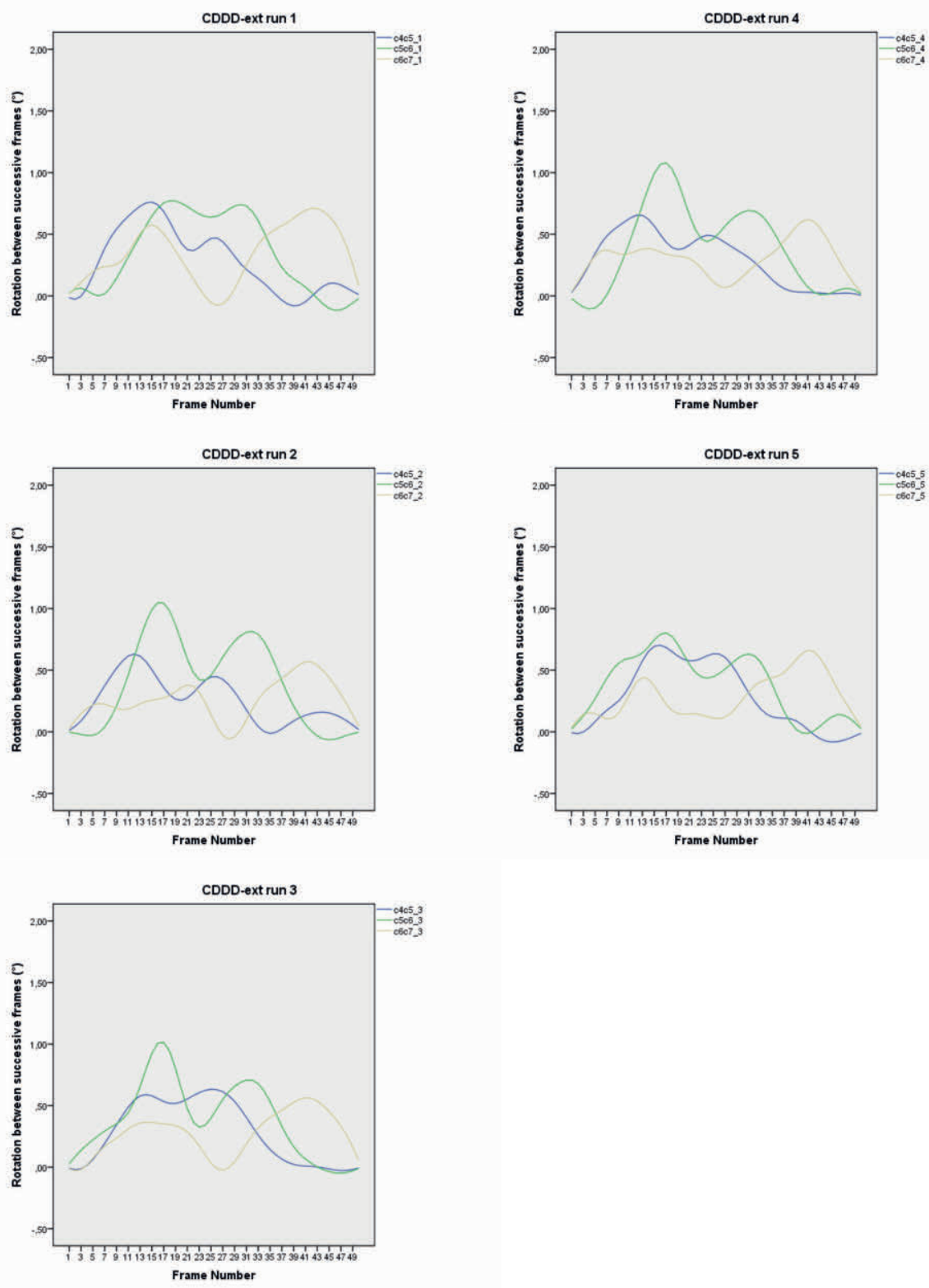

Figure S2: graphs depicting rotation (in degrees, y-axis) in the lower cervical motion segments, as a function of the frame number (x-axis) of the recording (CDDD-ext). The five graphs each depict one of the five analysis runs that was performed to determine precision and repeatability. Although the peaks vary in height and shape, the location of the peaks with respect to each other, and therefore the sequence in which the motion segments contribute to the extension movement, is the same at the end of the extension movement in all five graphs. 


\section{Appendix 3: Extension graphs - healthy controls $\mathrm{HC} 1-10$ at $\mathrm{T} 1$}

Graphs depicting sagittal rotation in segments in the lower cervical spine (block C4-C7) during extension of the entire cervical spine, for all healthy controls in $\mathrm{HC1}-10$ and $\mathrm{HC} 11-20$, at both time points. Peaks in the graphs depict maximum contributions of a segment in that phase of extension of the entire cervical spine.

Graphs for $\mathrm{HC1}-10$ are presented in page 1 and 2 in the same order. Graphs for group HC11-20 are presented in page 3 and 4 , also in the same order.

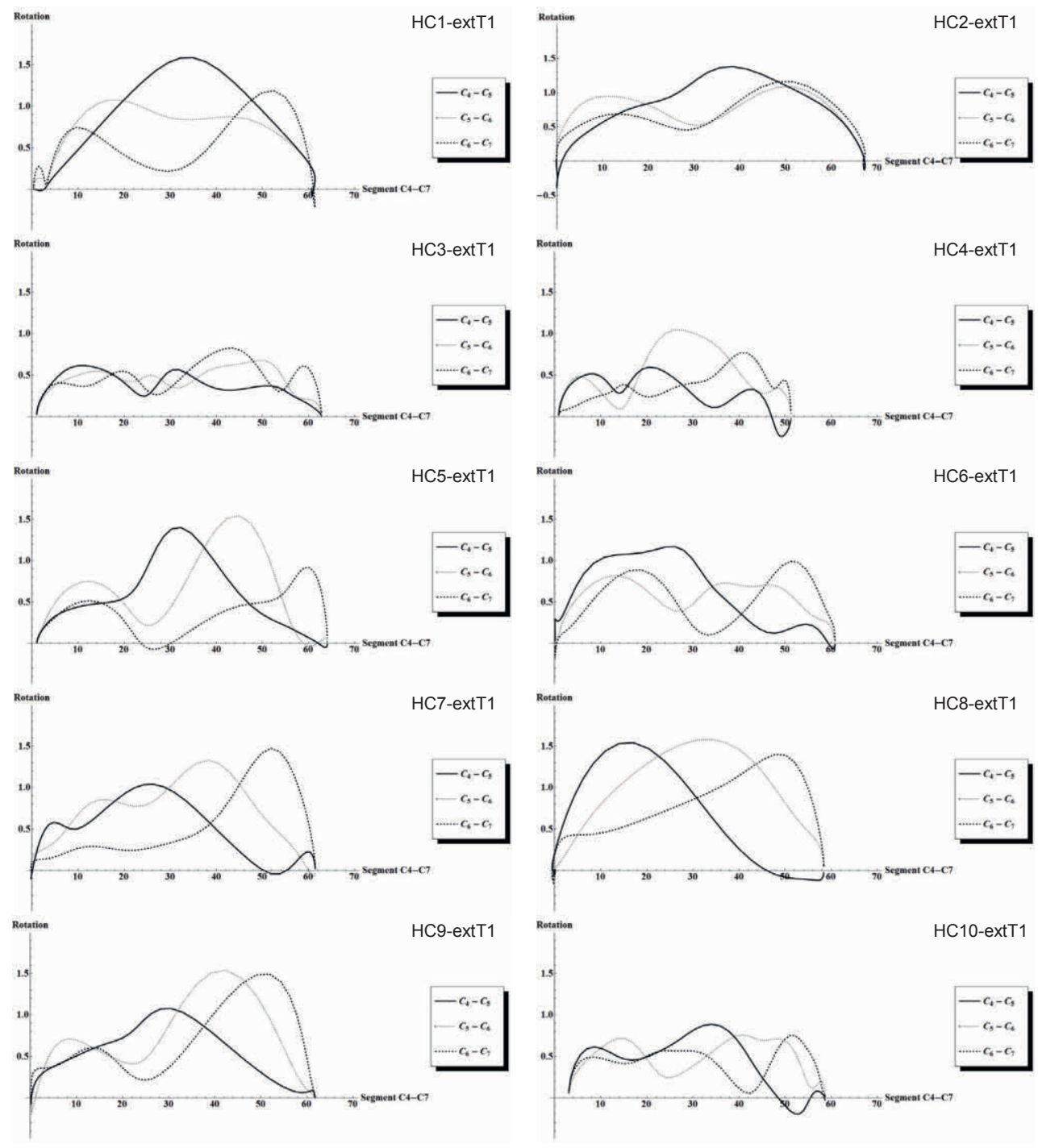


Chapter 4

Appendix 3: Extension graphs - healthy controls HC1-10 at T2

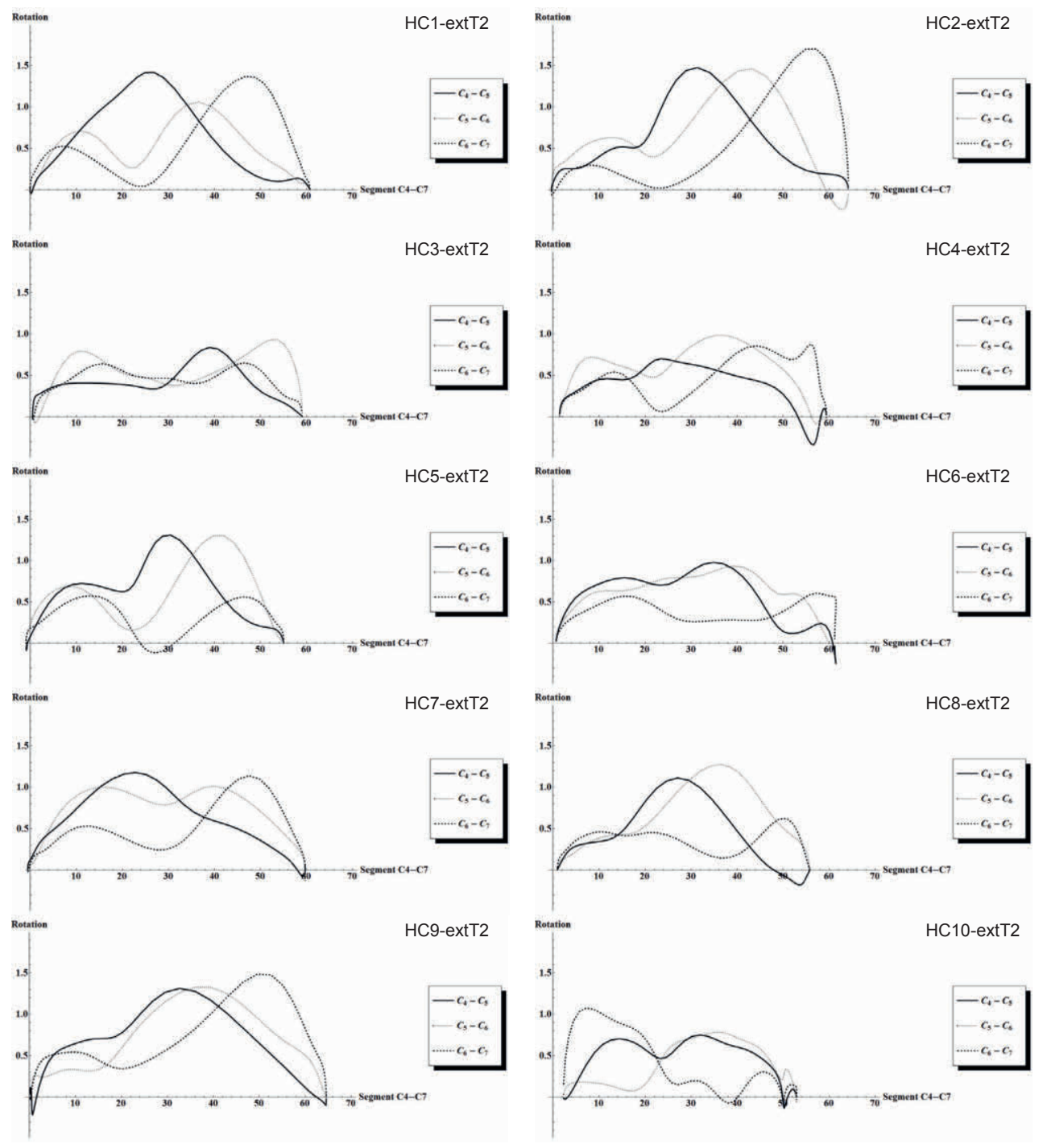


Appendix 3: Extension graphs - healthy controls HC11-20 at T1

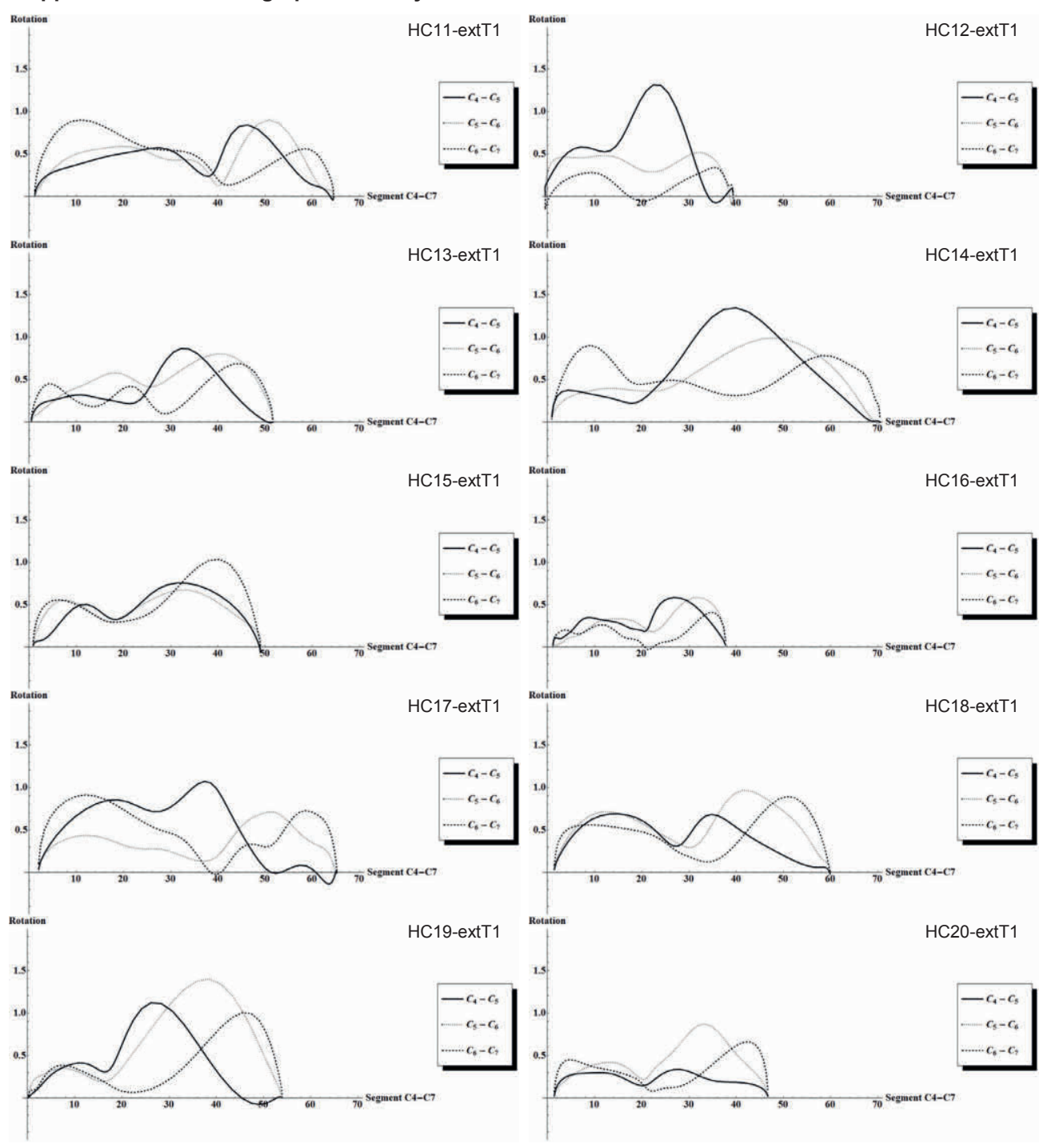


Chapter 4

Appendix 3: Extension graphs - healthy controls HC11-20 at T2

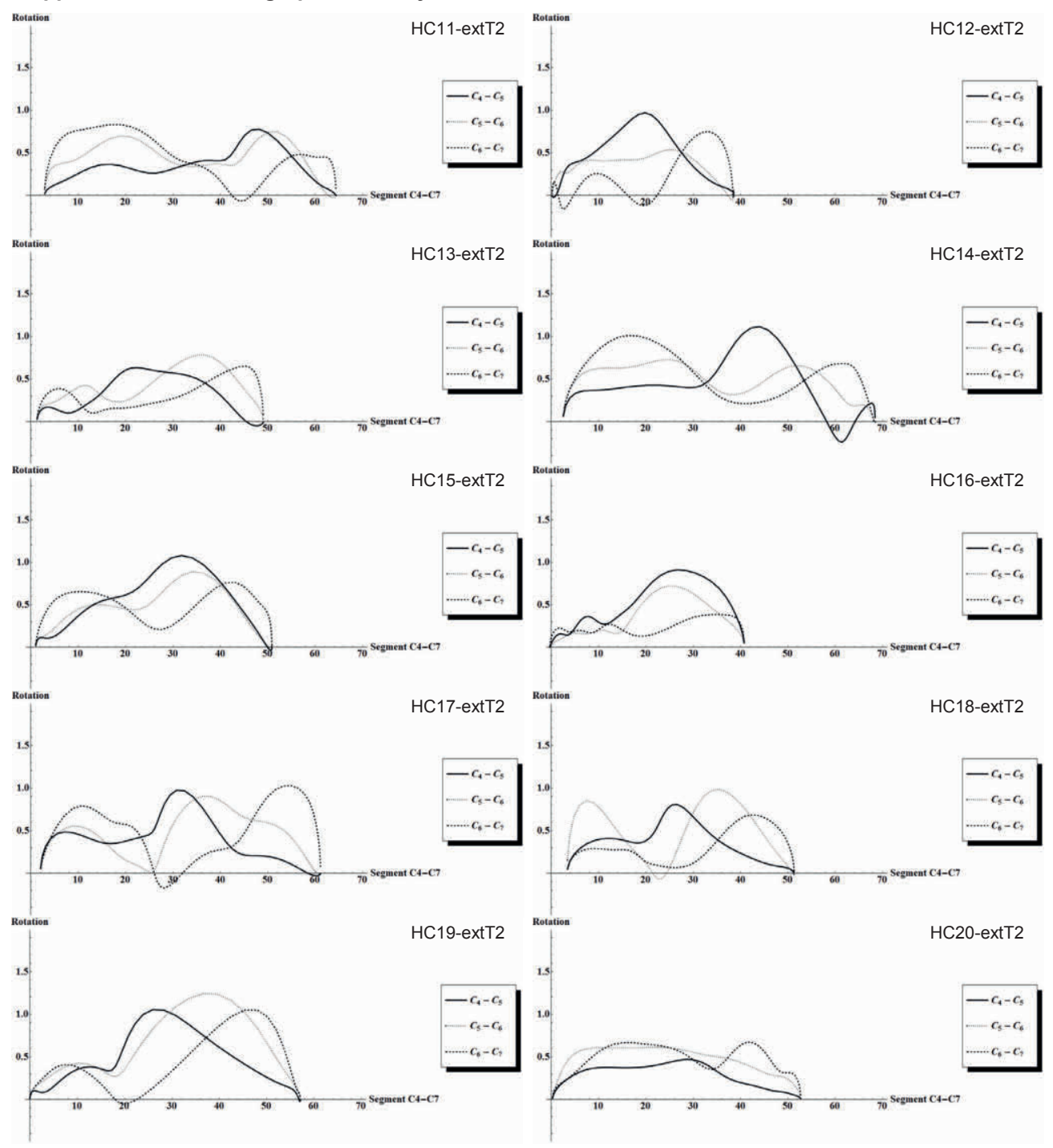




\section{Appendix 4 - Definition of normal extension}

Sagittal rotation ('Rotation', y-axis) of the individual motion segments of the lower cervical spine, set against cumulative sagittal rotation in the segment C4-C7 (i.e. the amount of extension in block $\mathrm{C} 4-\mathrm{C} 7$ ) is used to determine the sequence of segmental contribution to this cumulative rotation (example graph: Figure 1).

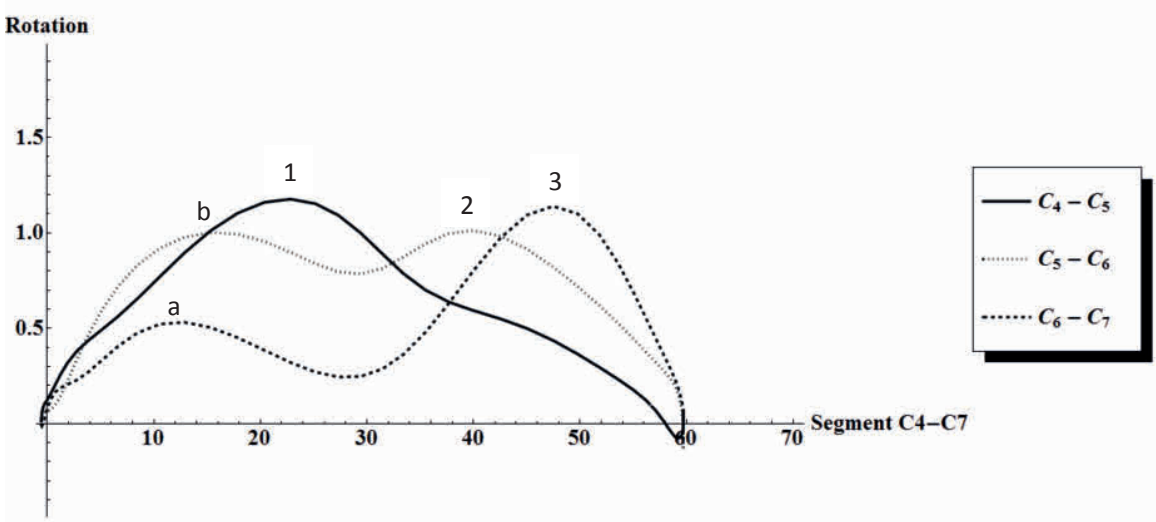

Figure 1: example graph depicting relative rotation in motion segments in the lower cervical spine during extension movement in $\mathrm{C} 4-\mathrm{C} 7$, in an asymptomatic subject. Peaks in the graphs depict maximum contributions of that motion segment. From left to right a peak in contribution of C6-C7 (a) is followed by a significant contribution of C5-C6 (b), and then C4-C5 (1). During the end of the extension movement the peak of C4-C5 (1) is followed by a peak in C5-C6 (2), and then C6-C7 (3) again. This last phase (1-2-3) is commonly found in extension in asymptomatic individuals $(85 \%)$. Only the order of the peaks is important, not the height of the peaks on itself, except for very small peaks with a rotation lower than 0.3 , which are deemed insignificant. In many cases there is a peak in C4-C5 at the end of extension, which is much smaller than the peak at (1), this is considered normal.

- Therefore, in normal extension towards the end of the extension movement (right side of the graph) the order 1-2-3 from the above graph (C4-C5, than C5-C6 and finally C6-C7) should be present.

- Very small peaks $(<0.3)$ are deemed to fall within measurement error.

- A small peak in C4-C5 at the end of extension is normal

- The first part of the extension movement is variable (left part of the graph). 


\section{Chapter 4}

\section{Appendix 5: Extension graphs - CDDD patients}

Graphs depicting sagittal rotation in segments in the lower cervical spine (block C4-C7) during extension of the entire cervical spine, for ten CDDD patients (CDDD1-CDDD10). Peaks in the graphs depict large contributions of a segment in that phase of extension of the entire cervical spine.
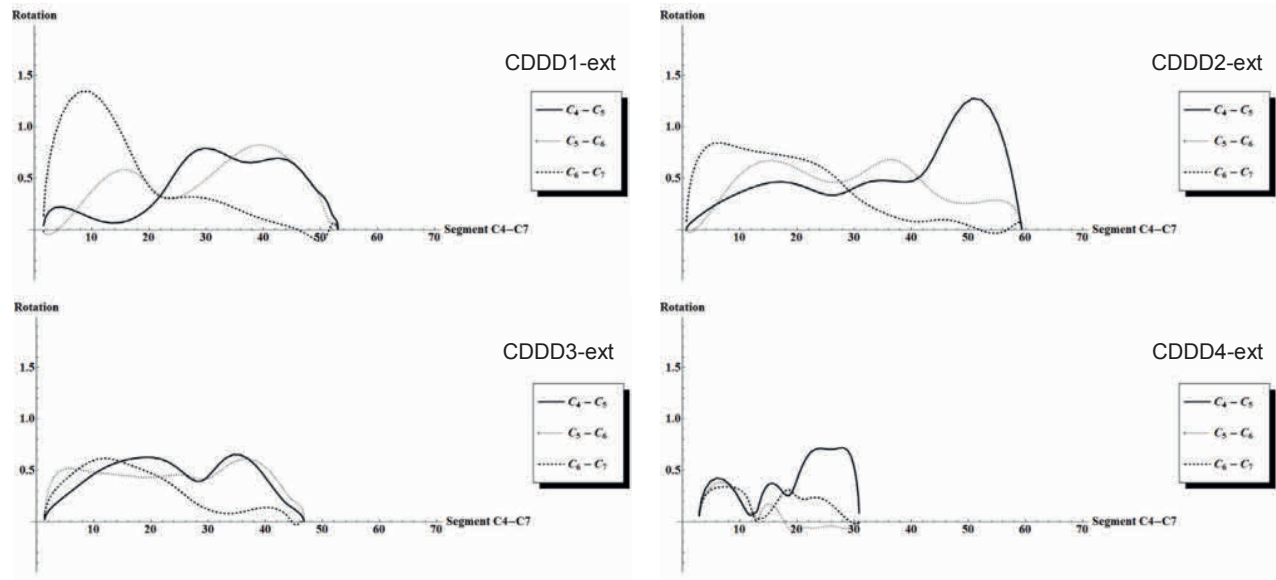

CDDD4-ext
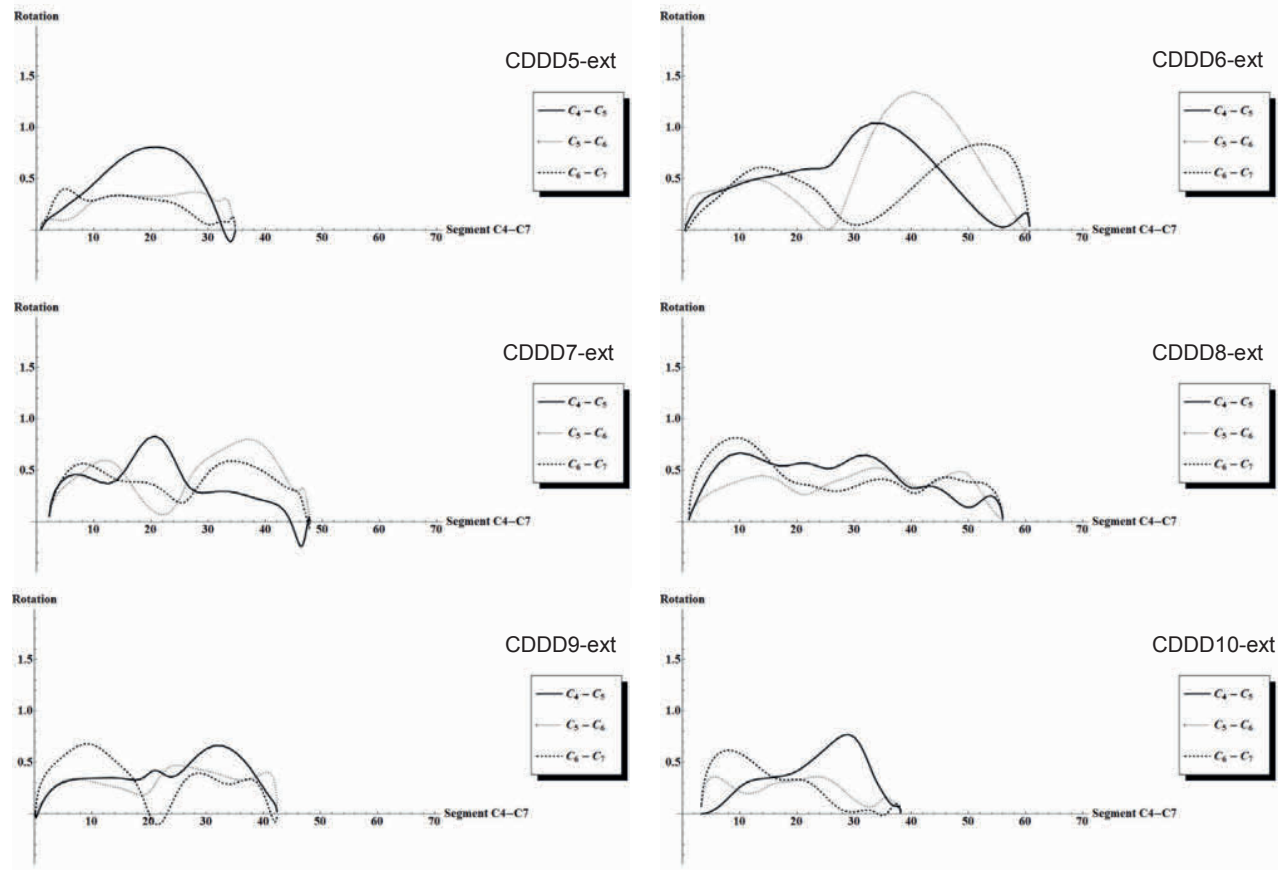


\section{Appendix 6: sROMS for extension studies used in the reliability analysis, compared to normal ranges in literature}

sROM for the extension study used in the reliability study, for all healthy controls in HC1-10, HC11-20, and all CDDD patients in CDDD1-10. All values are compared to the ranges described in literature (where range is defined as the average $+/$ - 2SD for those populations).

\begin{tabular}{|ccccc|}
\hline Healthy controls & C4-C5 & $\begin{array}{c}\text { sROM }\left(^{\circ} \text { ) }\right. \\
\text { C5-C6 }\end{array}$ & C6-C7 & $\begin{array}{c}\text { Segments within normal } \\
\text { range, } \mathbf{N} \text { (\%) }\end{array}$ \\
\hline HC1-extT1 & 22,8 & 21,0 & 16,5 & $3(100 \%)$ \\
HC2-extT2 & 22,4 & 22,6 & 19,6 & $3(100 \%)$ \\
HC3-extT1 & 17,3 & 20,1 & 21,9 & $3(100 \%)$ \\
HC4-extT1 & 11,9 & 21,5 & 17,0 & $3(100 \%)$ \\
HC5-extT1 & 22,3 & 23,1 & 17,6 & $3(100 \%)$ \\
HC6-extT2 & 19,5 & 21,9 & 19,1 & $3(100 \%)$ \\
HC7-extT1 & 17,7 & 24,1 & 19,8 & $3(100 \%)$ \\
HC8-extT2 & 14,3 & 24,2 & 16,3 & $3(100 \%)$ \\
HC9-extT2 & 19,2 & 21,1 & 24,2 & $3(100 \%)$ \\
HC10-extT1 & 16,4 & 20,6 & 18,6 & $3(100 \%)$ \\
HC11-extT1 & 15,3 & 18,4 & 21,2 & $3(100 \%)$ \\
HC12-extT2 & 18,3 & 9,9 & 8,5 & $3(100 \%)$ \\
HC13-extT1 & 15,0 & 20,5 & 15,8 & $3(100 \%)$ \\
HC14-extT2 & 18,0 & 20,7 & 24,7 & $3(100 \%)$ \\
HC15-extT1 & 14,1 & 15,8 & 18,2 & $3(100 \%)$ \\
HC16-extT1 & 13,3 & 12,0 & 7,4 & $3(100 \%)$ \\
HC17-extT2 & 18,4 & 18,6 & 22,1 & $3(100 \%)$ \\
HC18-extT2 & 16,5 & 18,1 & 13,4 & $3(100 \%)$ \\
HC19-extT1 & 15,5 & 21,7 & 15,5 & $3(100 \%)$ \\
HC20-extT2 & 11,4 & 19,5 & 20,5 & $3(100 \%)$ \\
& & & & \\
\hline \hline Mean & 16,4 & 19,5 & 17,9 & \\
SD & 2,7 & 3,7 & 4,6 & \\
\hline
\end{tabular}

\begin{tabular}{|ccccc|}
\hline CDDD patients & C4-C5 & $\begin{array}{c}\text { sROM }\left(^{\circ} \mathbf{~}\right. \\
\mathbf{C 5 - C 6}\end{array}$ & $\mathbf{C 6 - C 7}$ & $\begin{array}{c}\text { Segments within normal } \\
\text { range, } \mathbf{N}(\%)\end{array}$ \\
\hline CDDD1 & 17,6 & 17,4 & 16,6 & $3(100 \%)$ \\
CDDD2 & 23,7 & 18,4 & 16,6 & $3(100 \%)$ \\
CDDD3 & 16,8 & 18,4 & 10,3 & $3(100 \%)$ \\
CDDD4 & 17,3 & 3,0 & 7,8 & $2-3(67-100 \%)$ \\
CDDD5 & 13,8 & 10,7 & 9,5 & $3(100 \%)$ \\
CDDD6 & 20,9 & 20,5 & 18,8 & $3(100 \%)$ \\
CDDD7 & 12,4 & 18,2 & 15,1 & $3(100 \%)$ \\
CDDD8 & 21,4 & 16,3 & 17,9 & $3(100 \%)$ \\
CDDD9 & 15,5 & 13,9 & 12,9 & $3(100 \%)$ \\
CDDD10 & 15,0 & 9,7 & 10,2 & $3(100 \%)$ \\
& & & & \\
Mean & 17,4 & 14,7 & 13,6 & \\
SD & 3,4 & 5,1 & 3,7 & \\
\hline
\end{tabular}

\begin{tabular}{|llcl|}
\hline \multicolumn{1}{|c}{ Literature } & \multicolumn{3}{c|}{ Mean sROM ( ${ }^{\circ}$, SD) } \\
& C4-C5 & C5-C6 & C6-C7 \\
\hline Aho et al & $22(4)$ & $28(4)$ & $15(4)$ \\
Bhalla et al & $23(1)$ & $19(1)$ & $18(3)$ \\
Lind et al & $16(6)$ & $15(8)$ & $11(7)$ \\
Dvorak et al & $19(4)$ & $20(4)$ & $19(4)$ \\
& & & \\
& & & \\
From: Bogduk and Mercer '00 & & \\
\hline
\end{tabular}

Normal range is based on the data available in literature, as average +/- 2SD, depicted in the lower table. 



\section{Chapter}

Cervical spine kinematics after anterior cervical discectomy with or without implantation of a mobile cervical disc prosthesis; an RCT 


\section{Abstract}

\section{Background:}

When surgically treating cervical degenerative disc disease, the most commonly performed procedure is anterior cervical discectomy. This procedure is performed with, or without fusion promoting methods. For both options the rate of fusion is high and there is much debate whether fusion of the treated segment is a contributing factor to accelerated degeneration of adjacent motion segments. In an effort to prevent degeneration of adjacent segments (ASDeg) due to loss of mobility at the operated level, cervical disc arthroplasty (CA) was introduced. To evaluate the effectiveness of CA in preventing ASDeg long term studies are necessary. However, prevention of ASDeg is based on the premise that mobile disc prostheses preserve cervical spine motion in a physiological way. In this article the authors describe a short term protocol for a study that aims to investigate whether CA reaches the intended goal: restoration or preservation of physiological cervical spine motion. To this end, a technique is used to establish the sequence of contributions of cervical motion segments to flexion/extension of the spine.

\section{Methods:}

24 subjects between 18 and 55 years old, with radicular symptoms due to a herniated disc between $\mathrm{C} 5$ and $\mathrm{C} 7$, refractory to conservative therapy are randomized to simple discectomy, or CA. These groups are preceded by a pilot group of three subjects receiving CA. Fluoroscopic flexion-extension recordings are acquired preoperatively, and at three and 12 months postoperative. At these same time points, patient reported outcomes are collected, and a neurological examination is performed by and independent physician.

\section{Discussion:}

Studies investigating arthroplasty determine mobility by measuring segmental range of motion (sROM), which gives no information other than presence, and quantity, of mobility. SROM suffer from high variability. The authors therefore chose to use a method previously used in healthy controls, to describe the dynamic process of cervical spine motion in more detail. Determining cervical spine motion patterns has been reported to be more consistent than sROM. If a physiological motion pattern is absent after surgery in the CA group, prevention of future ASDeg is less likely. Radiological outcomes will be correlated to clinical outcomes.

\section{Trial Registration:}

NCT00868335

\section{Keywords:}

Cervical disc arthroplasty, cervical degenerative disc disease, fusion, cervical kinematics, randomized controlled trial. 


\section{Background}

Cervical degenerative disc disease is defined as complaints due to degenerative changes of the intervertebral disc and the intervertebral disc space. Herniation of the intervertebral disc itself or surrounding osteophytes cause radiculopathy due to compression of a nerve root, leading to pain and/or sensory and/or motor deficit. It can also lead to compression of the spinal cord itself causing myelopathy. Surgery can be considered if there is insufficient relief of symptoms with conservative treatment options, such as physical therapy, non-steroidal anti-inflammatory drugs, or immobilisation. Goal of surgery is relieving radiating arm pain in case of radiculopathy and prevention of progressive neurological deficits in case of myelopathy. The most commonly performed procedure is the anterior cervical discectomy, which is being performed either with, or without fusion promoting methods (called 'fusion' and 'simple discectomy', respectively). Clinical results of these methods are similarly good. Both methods have a high rate of fusion, up to $80 \%$ in simple discectomy, and over 95\% in fusion [1-4].

There is much debate whether fusion is a contributing factor to accelerated degeneration of adjacent motion segments (adjacent segment degeneration, ASDeg) or not. When this degeneration causes complaints, it is called adjacent segment disease (ASDis). The annual risk of ASDis is reported to be $2.9 \%$ [5]. It is not clear if fusion is in fact the culprit, or if it is just progression of degeneration in an ageing spine [6-9].

In an effort to prevent degeneration of adjacent segments due to loss of mobility at the operated level, cervical disc arthroplasty (CA) was introduced. In CA, a mobile disc prosthesis is inserted during surgery to preserve mobility of the operated spinal segment. Short term clinical results for CA are comparable to fusion, which's results are comparable to simple discectomy $[4,10,11]$. Long term studies are necessary to evaluate their effectiveness in preventing ASDis [10, 12].

Prevention of ASDis is based on the premise that mobile disc prostheses preserve cervical spine motion in a physiological way. Motion of the cervical spine is commonly measured by means of flexion-extension radiographs, which give information about the segmental range of motion (SROM). It suffers from a high intra- and inter-individual variability, severely limiting its use as a tool for diagnosis and follow-up in the individual patient. The sequence in which motion segments contribute to the movement to maximum extension or flexion in the entire cervical spine has been reported to be a more consistent parameter in healthy controls [13-15]. Therefore, a randomized controlled study to investigate the influence of implanting a mobile disc prosthesis on motion in the cervical spine was designed, using analysis of the sequence of segmental contributions.

Primary objective of the study is to ascertain whether a mobile cervical disc prosthesis restores/preserves the sequence of segmental contributions that is present in healthy controls, in contrast to patients undergoing simple discectomy. Secondary goal is to evaluate the clinical results of CA with the prosthesis used, compared to simple discectomy, to determine if results are comparable to reported results for this type of surgery. 


\section{Methods}

\section{Subjects}

Patients referred to our neurosurgical department who are eligible for surgery will be informed about the study and, if willing to receive further information, are then referred to HvS and/or TB to receive detailed study information and to evaluate eligibility. They then have a minimum of one week before they are contacted to inquire if they have any remaining questions and if they are willing to participate.

We include patients with a radiculopathy due to, MRI confirmed, single level soft disc herniation at the C5-C6 or C6-C7 level, refractory to at least 12 weeks of conservative treatment. They must be able to actively make a full flexion to extension movement. If there is osseous degeneration at the target level a flexion/extension radiograph is made to be certain that the segment is not spontaneously fused, which is defined as cortical bridging or less than $2^{\circ}$ segmental range of motion. At the time of conception of the study there was insufficient information about safety in patients with myelopathy; patients with a myelopathy are therefore excluded. Other reasons for exclusion are degenerative changes at more than the target level, prior surgery or radiotherapy to the cervical spine, active infection, or a history of malignancy. Inclusion and exclusion criteria are depicted in Table 1.

Informed consent will be acquired from all participants. The study has been approved by the local institutional medical ethical committee (Medical Research Ethics Committee Maastricht UMC+, METC 06-1-098) and has been registered on clinicaltrials.gov (NCT00868335).

Table 1: inclusion and exclusion criteria for the RCT.

\begin{tabular}{|l|l|}
\hline Inclusion & Exclusion \\
\hline $\begin{array}{l}\text { Patients } \\
\text { Mondication for simple discectomy }\end{array}$ & $\begin{array}{l}\text { Patients } \\
\text { myelopathy } \\
\text { I'Hermitte sign positive }\end{array}$ \\
$\begin{array}{ll}\text { Patients and healthy controls } \\
18-55 \text { years old }\end{array}$ & $\begin{array}{l}\text { Patients and healthy controls } \\
\text { prior surgery to the cervical spine } \\
\text { insufficient knowledge of the Dutch language } \\
\text { pregnancy } \\
\text { Willingness to complively make maximal extension to maximal flexion movement and vice versa } \\
\text { Informed consent }\end{array}$ \\
$\begin{array}{l}\text { Healthy controls } \\
\text { Nective infection } \\
\text { immature bone }\end{array}$ \\
\end{tabular}

\section{Sample size calculation}

Sample size calculation is based on the presence or absence of fusion at the operated segment, as the primary outcome is expected to be directly correlated to the presence of fusion. Fusion is defined as $<2^{\circ}$ range of motion. At an $\alpha$ set at 0.05 , the power $(\beta)$ of $80 \%$ to detect a $40 \%$ difference in the amount of patients with $<2^{\circ}$ range of motion, the necessary number of participants per group is ten. At a projected loss to follow up of $20 \%$ 
the amount of participants needed is twelve in each group. We also include a pilot group of three patients that will all receive CA, which will precede the randomised groups, to limit the possible influence of a learning curve. The total number of patients that will be included is therefore 27.

\section{Patient allocation and blinding}

Each patient is allocated to either arthroplasty or simple discectomy by randomly drawing an unmarked opaque sealed envelope from a non-sequential stack. Randomization is performed in a 1:1 ratio without a fixed block size.

The patient is informed about the result of the randomisation at the first radiological examination moment, up to two days before surgery. The surgeon and other caregivers are not blinded. Assessment of the radiological data is not blinded, since absence or presence of a prosthesis is clearly visible. The clinical outcome data are collected by a blinded caregiver.

\section{Interventions}

All patients are operated in the same centre by a single experienced spine surgeon. The prosthesis used is the Activ-C (B. Braun Aesculap, Germany).

Surgery is performed under general anaesthesia, with the patient in a supine position and the neck in a neutral position to slight extension. Antibiotic prophylaxis is given at induction of anaesthesia. The location of the incision is verified using fluoroscopy. In case of poor visibility of the target level, the shoulders are retracted by elastic tape. A transverse incision is made on the right side of the neck. The anterior aspect of the cervical spine is approached medially to the carotid sheath. The level is verified during surgery using fluoroscopy. The vertebral bodies are then distracted using two distraction pins and a Caspar spreader. Using a microscope, a standard discectomy is performed. The posterior longitudinal ligament is opened to reveal any sequesters. Decompression is verified by probing of the neuroforamen.

In the simple discectomy group haemostasis is checked, a low-vacuum drain is placed and the wound is closed in two layers.

In the arthroplasty group the correct prosthesis size is chosen using a fitting-prosthesis, the position is controlled by lateral and anterior-posterior fluoroscopy, taking care to place it in the midline. Subsequently, the caudal endplate is prepared for the keel of the prosthesis and the prosthesis is placed. The definite position is checked using fluoroscopy in 2 directions. The wound is rinsed to clear any drilling material, haemostasis is checked, a low-vacuum drain is placed and the wound is closed in two layers. 


\section{Post-surgical care}

Antibiotic prophylaxis is continued for 24 hours in the arthroplasty group. Cervical spine $X$-rays are acquired the day after surgery and the wound drain is removed. Patients in both groups are encouraged to mobilise, and resume home activities and work as soon as possible. A collar is not prescribed and patients are not routinely referred to a physical therapist.

Patients in the arthroplasty group are prescribed indomethacin 50mg three times a day for a period of three weeks to prevent periprosthetic calcifications, unless they have a history of gastric or intestinal complaints, or previous adverse reactions to non-steroidal anti-inflammatory drugs (NSAIDs). Patients in the simple discectomy group are allowed to use NSAIDs.

\section{Radiological outcome measures}

Radiological outcome measures are evaluated pre-surgery (0-2 days), and at three and twelve months post-surgery.

Radiological outcome measures are:

- Absence or presence of normal sequence of segmental contributions to cervical spine movement, analysed through fluoroscopic flexion-extension recording (FFER). The method of data acquisition and analysis is described below.

- segmental range of motion (sROM) of the target level is measured

- the presence of fusion is defined by cortical bridging and/or SROM less than $<2^{\circ}$

\section{Radiological data acquisition}

Fluoroscopic recordings are made with a digital X-ray detector (Toshiba Infinix VC-i, Toshiba Medical Systems Corporation, Japan), capturing frames of $1024 \times 1024$ pixels, at 10 frames per second. The recordings are stored without compression. To acquire the FFER, the subject is placed on a stool, with his shoulders perpendicular to the digital $\mathrm{x}$ ray receptor plate. The shoulder nearest to it is placed directly against it. The subject is asked to move the head in maximal extension without moving the upper part of his body. As soon as the recording is started, the subject is instructed to move the head in the sagittal plane from maximal extension to maximal flexion, without moving the upper part of the body. The subject is then allowed to relax for a moment, and then the movement from maximal flexion to maximal extension is recorded. The subject is asked to perform each movement in about five to ten seconds. It is important that the subject's shoulders are kept as low as possible during fluoroscopy to ensure that all the cervical vertebrae are visible. 


\section{Radiological data processing}

The authors have developed custom software that uses image recognition algorithms to track the skull and cervical vertebrae during flexion and extension throughout these series of frames [16]. The software follows bony structures within user-defined template areas (splines) throughout all frames, using a best-fit principle to match normalized gradient field images. To define these template areas the user of the software draws polygons around all vertebrae and part of the skull on the median frame of the recording (Figure 1). After the software has completed tracking these structures they can be manually evaluated, and corrections can be made if necessary.

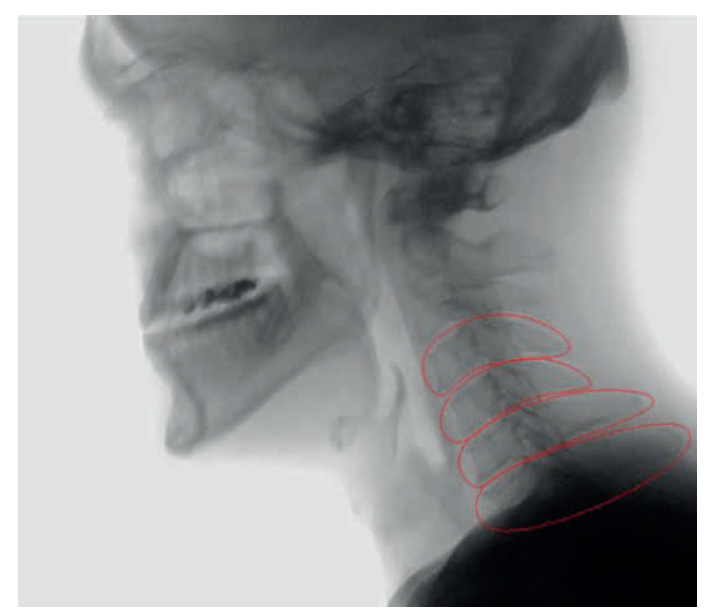

Figure 1: template area example; example of user defined template areas on the median frame of a FFER of a subject with cervical degenerative disc disease.

The rotational data between frames for each bony structure enables the user to calculate sROMs, but also sagittal rotation within a motion segment through time. The sequence of various segmental contributions to movement of the entire cervical spine can therefore be established (Figure 2). The sequence of segmental contributions is evaluated during full 'bending' extension-to-flexion, and vice versa. It is compared to ten healthy controls, and a historical control group [13-15]. 


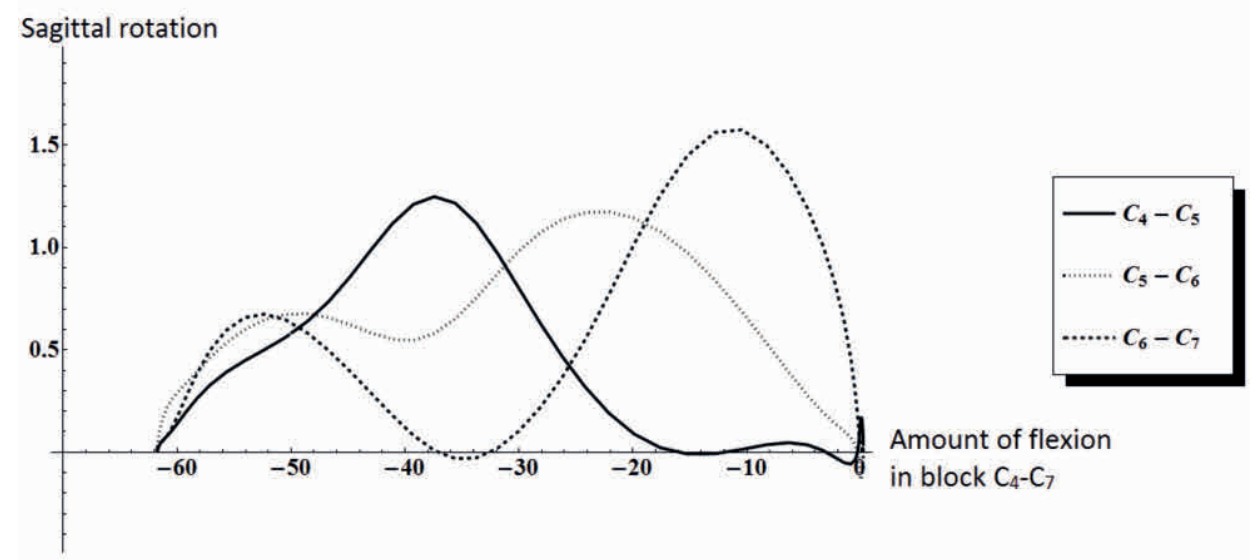

Figure 2: example graph; example graph depicting sagittal rotation in motion segments in the lower cervical spine during movement from maximal extension to maximal flexion in C4-C7 in an asymptomatic subject. Peaks in the graph depict maximum contributions of that motion segment at that moment in the flexion movement. From right to left, a peak in C6-C7 is followed by a peak in C5-C6, and then C4-C5. This is then followed by a second peak in C5-C6, and then in C6-C7. According to historical data this sequence is commonly seen in healthy controls.

\section{Clinical outcome measures}

At the same three time points at which the radiological analysis takes place the subject will be neurologically examined by an independent physician or physician assistant who is blinded to the treatment group. Muscle strength in myotomes C5-Th1 according to the Medical Research Council (MRC) Scale for Muscle Strength, sensory deficits in dermatomes C5-Th1, and deep tendon reflexes, are determined. Cervical spine motion is also estimated (flexion, extension, rotation, and lateral bending, estimated in degrees relative to the neutral position, i.e. the ears are aligned directly over the shoulders).

Adverse events (such as infection and re-operation) are registered, and the patients are asked to fill in the following questionnaires:

- Visual analogue scale for pain radiating to the arm (VAS-arm), on a $100 \mathrm{~mm}$ scale, ranging from 0 (no pain at all) to 100 (worst pain imaginable).

- Visual analogue scale for neck pain (VAS-neck), on a 100mm scale, ranging from 0 (no pain at all) to 100 (worst pain imaginable).

- Neck disability index (NDI), a 10-item questionnaire with a maximum score of 50 points ( 0 points being no perceived disability, and 50 points the maximum amount of disability), to assess perceived disability in work related, and non-work related activities due to neck complaints.

- Short-form 36 (SF36), a survey to assess general health, by using 36 questions to score 8 sections; physical functioning, social functioning, bodily pain, general health, vitality, mental health, and problems with daily activities due to either physical or emotional complaints. 
At the two postoperative time points (three and twelve months) one additional outcome is measured:

- Odom's outcome scale, a four-level outcome scale rating patients' outcome from poor to excellent depending on the level of resolution, improvement, or persistence of pre-operative symptoms.

\section{Statistical methods}

Dichotomous outcome measures (presence of normal sequence of segmental contributions, presence of fusion), and ordinal outcome measures (Odom's outcome scale) will be analysed using a $\chi 2$ analysis. Statistical significance will be defined as $P<0.05$ in the $\chi 2$ analysis. When the requirements of the $\chi 2$ analysis are not met (i.e. less than 5 samples per field) Fisher's exact test will be used.

Continuous outcome measures (NDI, VAS, SF36, sROM) will be analysed using an independent samples t-Test, after testing for normality. Statistical significance will be defined as $\mathrm{P}<0.05$ in the t-Test. If normality is not met, a Mann-Whitney $U$ test will be used.

All data will be analysed according to the 'intent-to-treat' principle, additionally a sensitivity analysis will be performed, based on presence or absence of fusion.

\section{Discussion}

The role of segmental fusion as a cause of ASDis, is widely discussed, and as the intended purpose of arthroplasty is prevention of ASDis, so is the indication for this type of treatment $[6,9,17]$. Given the fairly low incidence of ASDis, which has been reported to be about $2.9 \%$ annually, large studies with long term follow up are necessary to be able to determine the efficacy of arthroplasty to in fact prevent ASDis [8, 18]. As an alternative to these studies the authors decided to investigate the premise itself, that arthroplasty does in fact restore/preserve a normal sequence of segmental contributions in the cervical spine, while simple discectomy is likely to do not. Although a fused segment (in any of the two groups) will have no significant contribution, the sequence of the remaining segments is still of use. As this gives information about the influence of a fused segment on the rest of the cervical spine, and if there is a larger disturbance than simply a missing peak.

Many studies investigating arthroplasty determine mobility of the target level by measuring sROM before and after surgery, which allows for the conclusion that there is mobility, but gives no information other than the quantity of mobility. The authors therefore chose to use a method that was previously used in healthy controls to describe the dynamic process of cervical spine mobility in more detail, using the sequence of segmental contributions. This sequence has been reported to be much more consistent than 
sROMs, which are limited by high intra- and inter-individual variability [13-15]. Since the results are more consistent, sample size can be lower.

No results from a randomized controlled trial about the prosthesis used have been reported to date. Clinical outcome measures were therefore also included as secondary outcome measures, to evaluate if clinical results are in line with previous reports about other cervical disc prostheses.

As in almost all surgical studies, caregivers and X-ray analyses cannot be blinded, and patients report their own outcomes, therewith invoking risk of bias. This is a common weakness in this type of study, as blinding patients in these types of device studies is often regarded as not ethically appropriate, and most of the clinical outcomes are patient-reported outcomes [10]. As a result, many of the outcomes may suffer from bias, although the differences that have been reported so far were usually small [10]. The radiological outcome cannot be blinded completely since the prosthesis is clearly visible in the FFER, and although the motion analysis is performed by image recognition software, these analyses are checked manually to avoid errors. The clinical outcome data are collected by a blinded caregiver; since the patient is not blinded the possibility of the caregiver receiving information about the surgery is therefore present.

Sample size is small in this study. However, given the large expected difference in the amount of fusion between the groups, and the consistent findings when analysing sequence of segmental contributions, the authors expect to be able to draw conclusions about the primary outcome. For the secondary outcomes the sample size will be too small. However, the authors' haven previously shown that clinically relevant differences are not to be expected, and only aim to see if results are in line with those described in literature [10].

The study is industry sponsored, as are most of the RCTs on this subject [10]. This also can lead to bias. The influence of a conflict of interest on outcomes in trials investigating CA has recently been investigated, which led to the conclusion that while there was no influence on quality of life outcomes, it was associated with lower reported complication rates [12].

In the current study the sponsor has no influence on planning or conducting the study, no influence on data acquisition or analysis, or any aspect of the publication process.

The study has several strengths. First, the inclusion criteria are very strict. Inclusion of subjects with multiple degenerated segments would make it hard to distinguish between effects on the target level and adjacent levels due to the surgery, or due to pre-existent degenerative changes. Inclusion of only single level cases, without degenerative changes to the other levels, will therefore minimize confounding. Second, the method of radiological analysis gives more information than the traditionally made function radiographs. This allows for a more thorough analysis of mobility of the target level, as well as motion patterns of the cervical spine. This enables a clearer conclusion if cervical spine motion can be defined as physiological after arthroplasty, when compared to simple discectomy. As restoring normal mobility is the premise of the possible reduction of ASD through use 
of CA, this method of analysis therefore allows for and earlier indication if a specific type of prosthesis might be able to reduce ASD in the future.

\section{List of abbreviations}

RCT: randomized controlled trial, CA: cervical disc arthroplasty, ASD: adjacent segment degeneration, ASDeg: adjacent segment degeneration, ASDis: adjacent segment disease (= symptomatic adjacent segment degeneration), FFER: fluoroscopic flexion-extension recording, sROM: segmental range of motion, MRI: magnetic resonance imaging, MRC: medical research council, NDI: neck disability index, SF36: short form 36, VAS: visual analogue scale, NSAID: non-steroidal anti-inflammatory drug.

\section{Competing interests}

$H$. van Santbrink and T. Boselie have received reimbursements for conference attendance from the study sponsor (B. Braun Aesculap) in the past five years.

$\mathrm{R}$. de Bie, and $\mathrm{H}$. van Mameren report no competing interests, either financially, or otherwise.

\section{Acknowledgements}

The study is partly funded by B. Braun Aesculap, they have no influence on any aspect of conception/writing of the protocol, selection of subjects, data acquisition or data analysis, or any step in the writing or publication process of articles concerning this study.

The study is partly funded by a grant from Maastricht University Medical Center. They have no influence on any aspect of the study. 


\section{References}

1. Dowd, G.C. and F.P. Wirth, Anterior cervical discectomy: is fusion necessary? J Neurosurg, 1999. 90(1 Suppl): p. 8-12

2. Gore, D.R. and S.B. Sepic, Anterior discectomy and fusion for painful cervical disc disease. A report of 50 patients with an average follow-up of 21 years. Spine (Phila Pa 1976), 1998. 23(19): p. 2047-51.

3. Nandoe Tewarie, R.D., R.H. Bartels, and W.C. Peul, Long-term outcome after anterior cervical discectomy without fusion. Eur Spine J, 2007. 16(9): p. 1411-6.

4. Wirth, F.P., et al., Cervical discectomy. A prospective analysis of three operative techniques. Surg Neurol, 2000. 53(4): p. 340-6; discussion 346-8.

5. Hilibrand, A.S., et al., Radiculopathy and myelopathy at segments adjacent to the site of a previous anterior cervical arthrodesis. J Bone Joint Surg Am, 1999. 81(4): p. 519-28.

6. Seo, M. and D. Choi, Adjacent segment disease after fusion for cervical spondylosis; myth or reality? $\mathrm{Br} \mathrm{J}$ Neurosurg, 2008. 22(2): p. 195-9.

7. Nunley, P.D., et al., Symptomatic adjacent segment disease after cervical total disc replacement: re-examining the clinical and radiological evidence with established criteria. Spine J, 2013. 13(1): p. 5-12.

8. Helgeson, M.D., A.J. Bevevino, and A.S. Hilibrand, Update on the evidence for adjacent segment degeneration and disease. Spine J, 2013. 13(3): p. 342-51.

9. Saavedra-Pozo, F.M., R.A. Deusdara, and E.C. Benzel, Adjacent segment disease perspective and review of the literature. Ochsner J, 2014. 14(1): p. 78-83

10. Boselie, A., et al., Arthroplasty versus fusion in single-level cervical degenerative disc disease. Cochrane Database of Systematic Reviews, 2012. (in press, expected September 2012).

11. Barlocher, C.B., et al., Comparative evaluation of microdiscectomy only, autograft fusion, polymethylmethacrylate interposition, and threaded titanium cage fusion for treatment of single-level cervical disc disease: a prospective randomized study in 125 patients. Neurosurg Focus, 2002. 12(1): p. E4.

12. Alvin, M.D., et al., Cervical arthroplasty: a critical review of the literature. Spine J, 2014.

13. Van Mameren, H., Motion patterns in the cervical spine. 1988, Maastricht University.

14. Van Mameren, $H_{\text {., }}$ et al., Cervical spine motion in the sagittal plane (I) range of motion of actually performed movements, an X-ray cinematographic study. Eur J Morphol, 1990. 28(1): p. 47-68.

15. Bogduk, N. and S. Mercer, Biomechanics of the cervical spine. I: Normal kinematics. Clin Biomech (Bristol, Avon), 2000. 15(9): p. 633-48.

16. Reinartz, R., et al., Cervical vertebrae tracking in video-fluoroscopy using the normalized gradient field. Med Image Comput Comput Assist Interv, 2009. 12(Pt 1): p. 524-31.

17. Hilibrand, A.S. and M. Robbins, Adjacent segment degeneration and adjacent segment disease: the consequences of spinal fusion? Spine J, 2004. 4(6 Suppl): p. 190S-194S.

18. Harrod, C.C., et al., Adjacent segment pathology following cervical motion-sparing procedures or devices compared with fusion surgery: a systematic review. Spine (Phila Pa 1976), 2012. 37(22 Suppl): p. S96-S112. 


\section{Chapter}

\section{Sequence of segmental contributions in the caudal cervical spine in arthroplasty and ACD patients: a randomized controlled trial}

Toon FM Boselie, Henk van Mameren, Rob A de Bie, Henk van Santbrink submitted 


\section{Abstract}

Study Design:

Randomized controlled trial (RCT).

\section{Objective:}

To compare radiological outcomes (primarily the presence of a physiological motion pattern) and clinical outcomes for cervical arthroplasty (CA) and anterior cervical discectomy (ACD).

\section{Methods}

24 patients were randomized to either CA $(n=12)$ or ACD $(n=12)$. In each patient an extension cinematographic recording (ECR) was made before surgery and at three months and one year after surgery. In these recordings the sequence of segmental contributions (the sequence in which motion segments C4-C5, C5-C6 and C6-C7 contribute to extension in the block (4-C7) was evaluated, and compared to the sequence as described in asymptomatic individuals to check for the presence of a 'normal' sequence. At the same time points patient reported outcomes (VAS scores for arm pain and neck pain, Neck Disability Index and Short Form-36 scores) were collected, and patients were neurologically examined.

\section{Results}

In both groups ten patients were available for follow up at one year. At one year, fusion rate was $0 / 10(0 \%)$ in the CA group, and $7 / 10(70 \%)$ in the ACD group. The majority of the patients in the CA group (8/10,80\%) showed a normal sequence of segmental contributions. In the ACD subgroup with preserved motion $(n=3)$ there were two patients with a normal sequence of segmental contributions (20\% of the total ACD group). There were no differences in patient reported outcomes between the CA and ACD group.

\section{Conclusions}

In $80 \%$ of the CA group the prosthesis facilitates the sequence of segmental contributions in C4-C7 that is described in asymptomatic patients, while motion is preserved in all patients. There were no differences in patient reported outcomes between the treatment groups. If normal motion at the operated segment indeed prevents adjacent segment disease, this premise is met in the majority of the CA patients.

\section{Trial registration}

NCT00868335 


\section{Introduction}

Cervical arthroplasty (CA) by implanting a motion-preserving disc prosthesis is one of the options in the treatment of cervical degenerative disc disease (CDDD). The advantage of CA over anterior cervical discectomy (ACD) or anterior cervical discectomy with fusion (ACDF), should be prevention of symptomatic adjacent segment degeneration [1], or adjacent segment disease (ASDis), which has a reported incidence of $2.9 \%$ per year [2]. In biomechanical studies fusion of a motion segment led to increased intradiscal pressure and hypermobility in adjacent segments [3], this in turn was postulated to play a role in the development of ASDis [1]. Fusion rate at one year is high for ACD (70\%), and even higher for ACDF (95-100\%) [4-7].

In the short term, patient reported outcomes are similar for CA, anterior cervical discectomy (ACD), and anterior cervical discectomy and fusion (ACDF) [4, 8, 9]. Preliminary mid- to long-term evaluations also show similar clinical results [9-14].

The goal of CA should not only be to preserve mobility, but also to facilitate normal movement, which means a physiological interplay within the motion segments. This can be expressed as the sequence of segmental contributions during active flexion and/or extension of the entire cervical spine $[15,16]$. This sequence can be determined from a flexion cinematographic recording (FCR) and/or an extension cinematographic recording (ECR). The authors have previously described a specific sequence of segmental contributions in extension of the caudal cervical spine (C4-C7) that is present in asymptomatic individuals, which was used to define 'normal motion' [17]. Use of this definition has a high sensitivity, specificity and reliability in differentiating between asymptomatic individuals and patients with CDDD [17].

The aim of the current study was to apply this method of analysis of extension recordings in a randomized controlled trial comparing CA (Activ ${ }^{\circledR}$ cervical disc prosthesis, B. Braun/Aesculap, Tüttlingen, Germany) and ACD [18]. First, it was hypothesized that implanting the movable cervical disc prosthesis facilitates a normal sequence of segmental contributions, while ACD does not. Second, the clinical results of the two treatment modalities were compared.

\section{Materials and methods}

\section{Study design}

A prospective, randomized, controlled trial comparing CA using the ActivC ${ }^{\circledR}$ cervical disc prosthesis and ACD was performed [18]. A total of 24 patients with a cervical radicular syndrome corresponding to soft disc single level CDDD at C5-C6 or C6-C7 on magnetic resonance imaging (MRI), with symptoms refractory to at least 12 weeks of non-surgical treatment, and without degenerative signs (e.g. osteophytes) were included. The latter 
was excluded with an X-ray if deemed necessary. Detailed inclusion and exclusion criteria are shown in Table 1. Sample size calculation was based on the presence or absence of fusion at the operated segment, as the primary outcome (a normal sequence of segmental contributions) was expected to correlate directly with the presence of fusion. At an $\alpha$ set at 0.05 , the power $(\beta)$ of $80 \%$ to detect a $40 \%$ difference in the amount of patients with less than $2^{\circ}$ range of motion, the necessary number of participants per group was ten. At a projected loss to follow up of $20 \%$ the amount of participants needed was twelve in each group. Randomization was performed in a 1:1 ratio with no fixed block size using unmarked, non-sequential, sealed, opaque envelopes. Patients underwent surgery between December 2008 and September 2014. Patients and caregivers were not blinded to allocation. Written informed consent was acquired from all participants. The registered (NCT00868335) study was approved by the local institutional medical ethical committee. Clinical and radiological data were collected preoperatively, and at three months and one year after surgery. Radiological data at three months were not included in the current paper as they will be the focus of a separate paper investigating optimal timing of radiological follow up. Clinical data included neurological status, VAS scores for arm and neck pain, the Neck Disability Index (NDI), Short Form 36 (SF36), and Outcome according to Odom's outcome criteria. Radiological data included a sequence of segmental contribution analysis, which is described below in more detail, and assessment of the presence of fusion (defined as cortical bridging and/or a segmental range of motion (sROM) less than $2^{\circ}$ ). The trial design and surgical technique have been described in more detail previously [18].

Table 1: Inclusion and exclusion criteria for the study.

\begin{tabular}{ll}
\hline Inclusion criteria & Exclusion criteria \\
\hline $\begin{array}{l}\text { Symptomatic cervical degenerative disc disease } \\
\text { (radicular syndrome) }\end{array}$ & Myelopathy \\
Skeletally mature patients (18 years or older) & $\begin{array}{l}\text { Multiple levels of cervical degenerative disc disease / } \\
\text { spondylosis. }\end{array}$ \\
$\begin{array}{l}\text { Single level, soft disc herniation (C5-C6 or C6-C7) } \\
\text { Insufficient relief of pain after >12 weeks conservative }\end{array}$ & $\begin{array}{l}\text { Previous cervical spine surgery } \\
\text { treatment }\end{array}$ \\
Able to fill out the required questionnaires & Malignancy \\
Informed consent & Metabolic bone disease (e.g. osteoporosis) \\
& Inflammatory spinal arthritis \\
\hline
\end{tabular}

\section{Radiological data acquisition}

Fluoroscopic recordings of a maximum 'bending' flexion to maximum extension movement, and vice versa, were made with a digital X-ray detector as previously described [18]. The extension recordings were analyzed using previously described image recognition software to determine rotations and translations of all the cervical vertebrae 
throughout all video frames of the recording [19]. Using these data, graphs depicting sagittal rotation within each motion segment (C4-C5, C5-C6, and C6-C7) between each pair of successive frames were generated as a function of the cumulative extension in block C4-C7. Using these graphs, the sequence in which these segments contribute to extension in block C4-C7 can be visualized. Based on these graphs, motion in the caudal cervical spine was scored as 'normal' or 'not normal' for all patients, based on a previously published definition of the normal sequence of segmental contributions [17]. An example graph of a normal sequence of segmental contributions is shown in Figure 1.

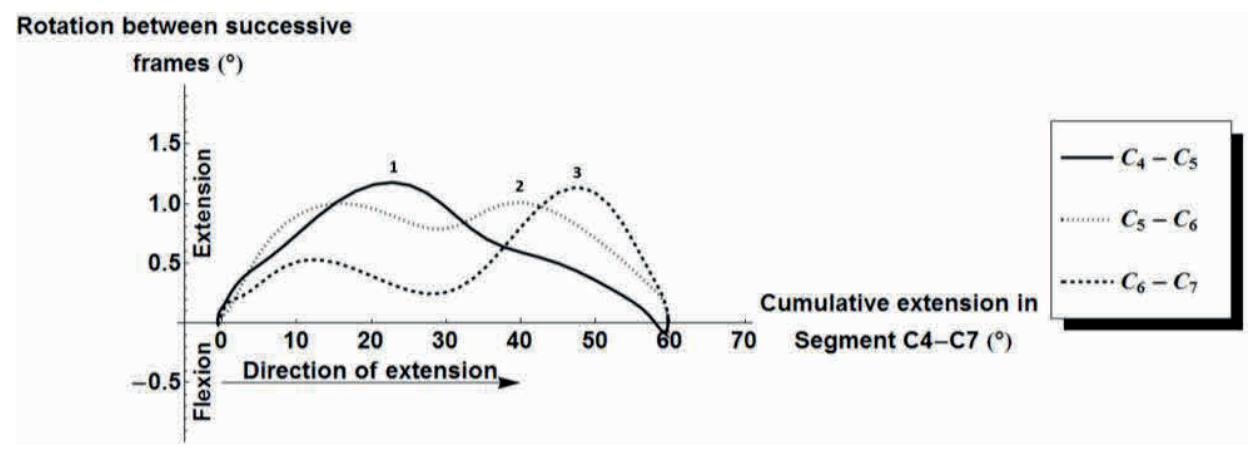

Figure 1: Example graph depicting sagittal rotation in segments in the lower cervical spine (block C4-C7) during extension of the entire cervical spine, in a healthy control. On the $y$-axis sagittal rotation between successive frames is shown. On the $\mathrm{x}$-axis cumulative extension in block C4-C7 is shown. Peaks in the graphs depict maximum contributions of a segment in that phase of extension of the entire cervical spine. From left to right a peak in contribution of C6-C7 is followed by a peak in contribution of C5-C6, and then C4-C5 (1). In the following phase, from about 25 up to 60 degrees of extension between C4 and C7 the peak of C4-C5 (1) is followed by a peak in C5-C6 (2), and then C6-C7 again (3). This last phase (1-2-3) is common in extension in healthy controls. All peaks should be above $0.3^{\circ}$ rotation between successive frames to be above measurement error, and therefore to be considered relevant. (From: "A Pilot Study Of Sequence Of Segmental Contributions In The Lower Cervical Spine During Active Extension And Flexion: Healthy Controls vs. Cervical Degenerative Disc Disease Patients" by Boselie TF, van Santbrink H, de Bie RA, van Mameren H., 2016, Spine (Phila Pa 1976), 42(11): E642E647. Copyright [2016] by Wolters Kluwer. Reprinted with permission.)[17].

\section{Statistical analysis}

All data were analyzed according to an intention-to-treat principle. Additionally, a sensitivity analysis was performed comparing all patients with preserved motion after surgery from both groups, versus all patients with a fused segment. Continuous data were analyzed using an independent samples t-test unless normality was not met, in which case a Mann-Whitney $U$ test was used. Dichotomous data were analyzed using a Chi-squared analysis. If the criteria for a Chi-squared analysis were not met (i.e. less than five expected samples per field) Fisher's exact test was used. For all tests, statistical significance was defined as $P<0.05[18]$. 


\section{Results}

Inclusion and follow-up

Twenty-six patients were referred to the investigators to be assessed for eligibility, two of these were not included (both due to degenerative changes at other levels than the index level). All patients received the allocated treatment. In both groups, ten out of 12 subjects (83\%) were available for follow up one year after surgery (Figure 2).

\section{Study Flow Diagram}

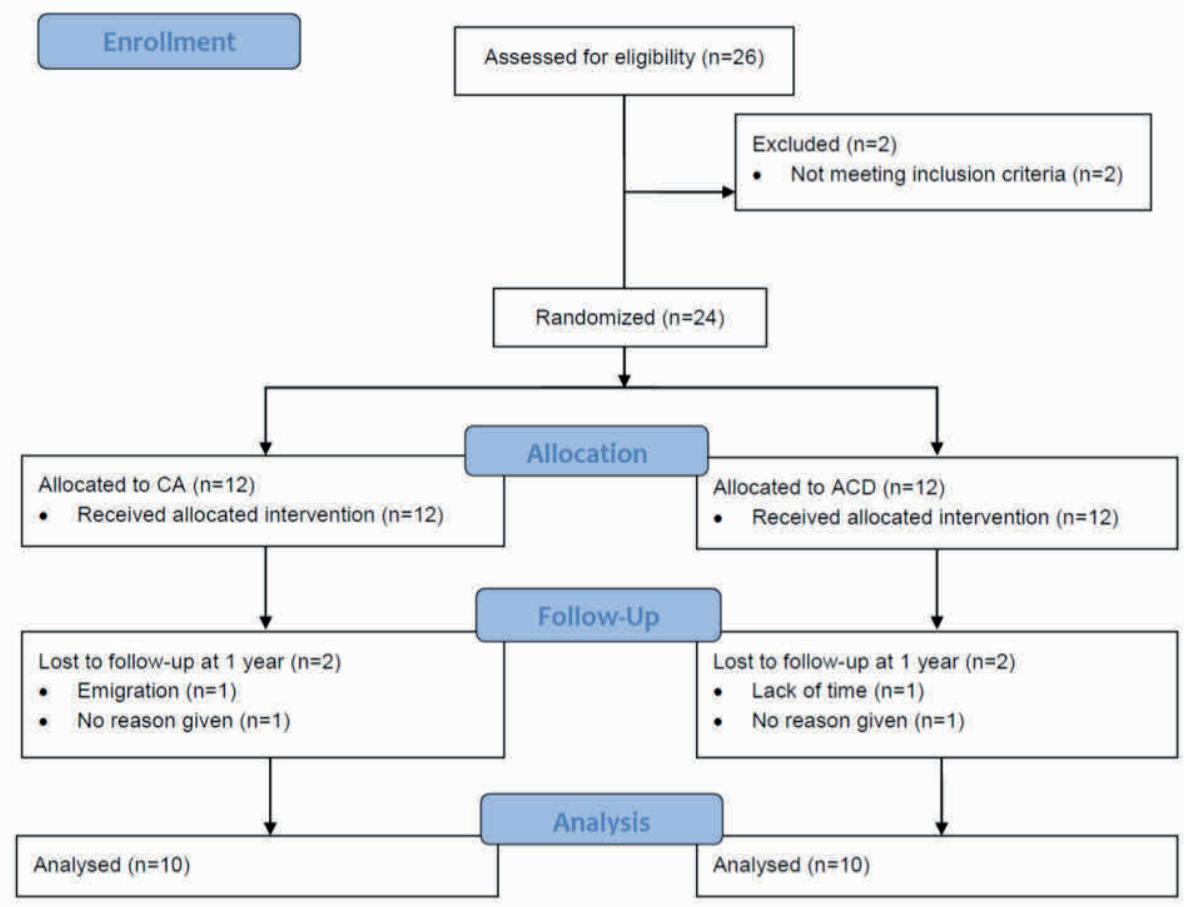

Figure 2: Study flow diagram according to CONSORT guidelines.

\section{Primary radiological outcomes}

Sequence of segmental contributions and preservation of motion

Preoperatively, 2/12 (17\%) patients in the CA group and 5/12 (42\%) patients in the ACD group had a normal sequence of segmental contributions $(P=0.371)$ (Table 2$)$. At one year 
after surgery $8 / 10(80 \%)$ showed a normal sequence of segmental contributions in the CA group versus $2 / 10(20 \%)$ in the ACD group ( $P=0.007)$. The two patients in the CA group with a normal sequence of segmental contributions preoperatively, still had a normal sequence at one year (Appendix 1 and Appendix 2). Motion was preserved in only three patients in the ACD group (30\%), while in all of the patients in the CA group (100\%) $(P=0.003)$. In the subgroup of ACD patients with preserved motion at the index level $(n=3)$, there were two patients with a normal sequence of segmental contributions (Table 2), one of these also had a normal sequence preoperatively. Representative graphs from the CA group and the ACD group are shown in Figure 3, the graphs from all patients are available in Appendix 1.

Table 2: Motion before and after surgery. Before surgery there was no significant difference in the amount of patients with a normal sequence of segmental contributions. At one year the amount of patients with a normal sequence was significantly higher in the CA group. At one year, there were no CA patients with a fused segment after surgery. There were 7 out of 10 patients with a fused segment in the ACD group. Mean sROM was significantly lower at 1 year after surgery in the ACD subgroup with a fused segment.

\begin{tabular}{|c|c|c|c|}
\hline & Normal sequence $(n, \%)$ & sROM (Mean, SD, ${ }^{\circ}$ ) & Fusion ( $\mathrm{n}, \%)$ \\
\hline \multicolumn{4}{|l|}{ Baseline } \\
\hline$C A(n=12)$ & $2 / 12(16.7 \%)$ & $11.4(4.7)$ & $0 / 12(0 \%)$ \\
\hline $\operatorname{ACD}(n=12)$ & $5 / 12(41.7 \%)$ & $14.9(4.8)$ & $0 / 12(0 \%)$ \\
\hline p-value & $P=0.371^{*}$ & $\mathrm{P}=0.088^{* *}$ & $P=1.000$ \\
\hline \multicolumn{4}{|l|}{1 year } \\
\hline$C A(n=10)$ & $8 / 10(80 \%)$ & $12.3(6.1)$ & $0 / 10(0 \%)$ \\
\hline ACD (all, n=10) & $2 / 10(20)$ & $3.0(3.6)$ & $7 / 10(70 \%)$ \\
\hline $\begin{array}{l}\text { - ACD with preserved motion } \\
\qquad(n=3)\end{array}$ & $2 / 3(67 \%)$ & $7.7(3.7)$ & \\
\hline \multicolumn{2}{|c|}{ - ACD with fused segment $(n=7) 0 / 7(0 \%)$} & $1.1(0.4)$ & \\
\hline p-value & $\mathrm{P}=0.007^{*}$ & $P=0.001 * *$ & $\mathrm{P}=0.003 *$ \\
\hline
\end{tabular}

* CA vs. ACD (all), Fisher's exact

${ }^{* *}$ CA vs. ACD (all), Mann-Whitney U 
Rotation between successive
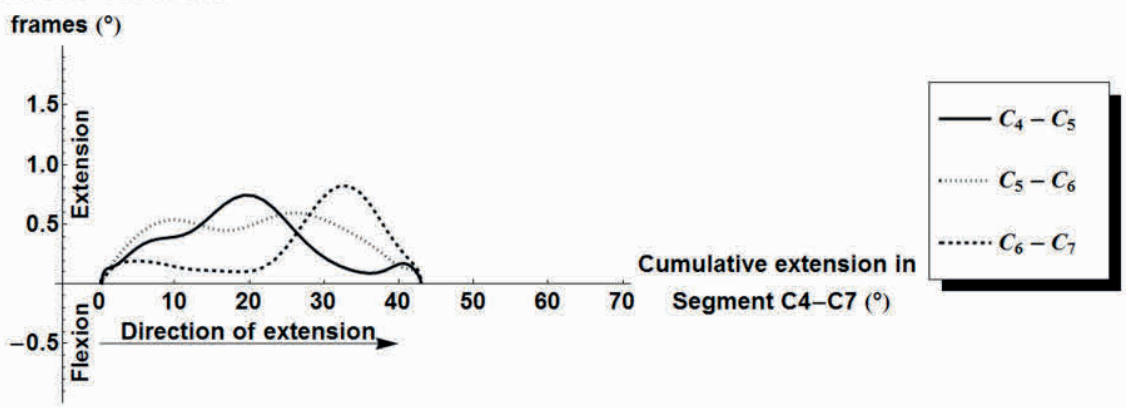

\section{Rotation between successive}

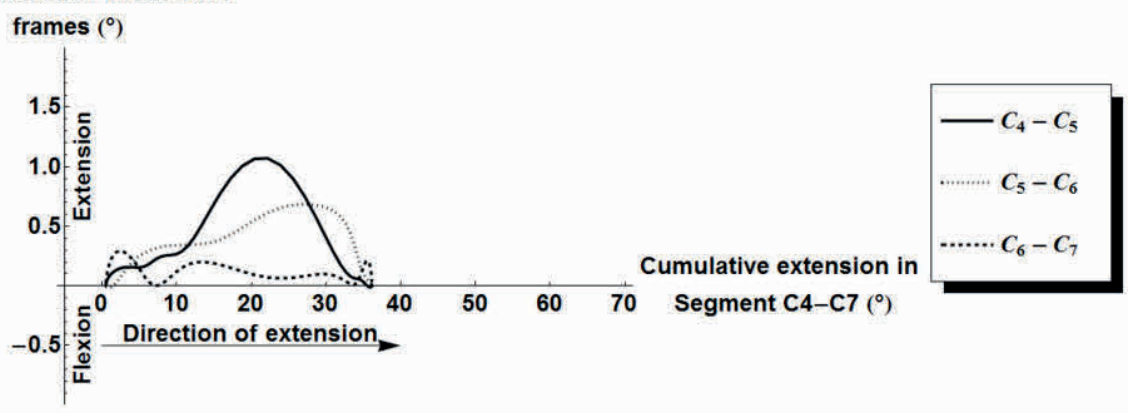

Figure 3: Representative examples of the postoperative graph at one year after surgery. Representative examples of the postoperative graph at one year after surgery of a CA patient (top graph, patient 9, operated at C6C7), and an ACD patient (bottom graph, patient 11, operated at C6-C7). The sequence of segmental contribution in the last phase of extension in the CA patient is comparable to the sequence found in asymptomatic subjects. This is not the case in the ACD patient, where a peak is missing from the sequence due to the fusion in C6-C7.

\section{Clinical outcomes}

\section{Patient reported outcomes}

There were no significant differences in any of the outcomes at baseline (Table 3). At three months and one year follow up there were no significant differences for any of the patient reported outcomes (Tables 4 and 5). Patients in both treatment arms had on average a significant improvement in patient reported outcomes compared to baseline (Table 6). 
Table 3: Baseline characteristics for both treatment groups. There were no significant differences between the two groups.

\begin{tabular}{llll}
\hline & CA & ACD & P-value \\
\hline Female gender $(n)$ & $8 / 12(67 \%)$ & $7 / 12(58 \%)$ & $1.000^{*}$ \\
Age (mean years, SD) & $41.3(8.5)$ & $42.0(5.7)$ & $0.823^{* *}$ \\
Level C5-C6 vs. C6-C7 & $9: 3$ & $6: 6$ & $0.400^{*}$ \\
NDI (Mean, SD) & $57.2(18.9)$ & $57.6(19.2)$ & $0.954^{* *}$ \\
VAS arm (Mean, SD) & $66.2(18.4)$ & $65.3(21.7)$ & $0.913^{* *}$ \\
VAS neck (Mean, SD) & $59.8(29.6)$ & $57.0(28.4)$ & $0.814^{* *}$ \\
SF36-MCS (Mean, SD) & $35.6(12.0)$ & $38.0(13.9)$ & $0.658^{* *}$ \\
SF36-PCS (Mean, SD) & $38.3(5.4)$ & $37.6(8.1)$ & $0.804^{* *}$ \\
\hline
\end{tabular}

* Fisher's exact

** t-test

Table 4: clinical results three months after surgery. There were no significant differences for the patient reported outcomes.

\begin{tabular}{llll}
\hline & CA & ACD & P-value \\
\hline NDI (Mean, SD) & $35.1(12.7)$ & $39.7(21.2)$ & $0.869 *$ \\
VAS arm (Mean, SD) & $15.5(31.8)$ & $18.8(26.3)$ & $0.946^{*}$ \\
VAS neck (Mean, SD) & $26.9(27.8)$ & $25.7(34.2)$ & $0.638 *$ \\
SF36-MCS (Mean, SD) & $44.9(11.8)$ & $37.5(16.9)$ & $0.309 *$ \\
SF36-PCS (Mean, SD) & $44.2(8.4)$ & $44.2(8.0)$ & $0.990 * *$ \\
Outcome according to Odom's & & & \\
criteria & & $1(9 \%)$ & \\
- Excellent & $6(55 \%)$ & $7(64 \%)$ & \\
- Good & $4(36 \%)$ & $2(18 \%)$ & \\
- Fair & $1(9 \%)$ & $1(9 \%)$ & \\
- Poor & $0(0 \%)$ & $8(73 \%)$ & \\
- Success (Good+Excellent) & $10(91 \%)$ & & \\
Adverse events & & $0 / 11(0 \%)$ & \\
- Wound infections & $0 / 11(0 \%)$ & $0 / 11(0 \%)$ & \\
- Other infections & $0 / 11(0 \%)$ & n.a. & \\
- Implant migration & $0 / 10(0 \%)$ & $2 / 11(18 \%)$ & \\
- Neurological worsening & $0 / 11(0 \%)$ & $0 / 11(0 \%)$ & \\
- Neurological worsening (mye- & $0 / 11(0 \%)$ & & \\
lopathy) & & & \\
\hline
\end{tabular}

* Mann-Whitney U

$* *$ t-test

*** Fisher's exact 
Table 5: Results one year after surgery. There were no significant differences for the patient reported outcomes.

\begin{tabular}{llll}
\hline & CA & ACD & P-value \\
\hline NDI (Mean, SD) & $33.8(14.9)$ & $31.1(19.5)$ & $0.761 * *$ \\
VAS arm (Mean, SD) & $6.8(8.1)$ & $18.8(29.0)$ & $0.699 * *$ \\
VAS neck (Mean, SD) & $32.2(37.8)$ & $24.9(37.9)$ & $0.540 * *$ \\
SF36-MCS (Mean, SD) & $44.8(11.8)$ & $44.3(13.7)$ & $0.880 * *$ \\
SF36-PCS (Mean, SD) & $44.3(10.9)$ & $49.2(9.1)$ & $0.284 * * *$ \\
Outcome according to & & & \\
Odom's criteria & & $4(40 \% 0$ & \\
- Excellent & $3(30 \%)$ & $3(30 \%)$ & $0.211 * * * *$ \\
- Good & $7(70 \%)$ & $2(20 \%)$ & \\
- Fair & $0(0 \%)$ & $1(10 \%)$ & \\
- Poor & $0(0 \%)$ & $7(70 \%)$ & \\
- Success (Good+Excellent) & $10(100 \%)$ & & \\
Adverse events & & $0 / 10(0 \%)$ & \\
- Wound infections & $0 / 10(0 \%)$ & $0 / 10(0 \%)$ & \\
- Other infections & $0 / 10(0 \%)$ & n.a. & \\
- Implant migration & $0 / 10(0 \%)$ & $3 / 10(30 \%)$ & \\
- Neurological worsening & $1 / 10(10 \%)$ & $0 / 10(0 \%)$ & \\
- Neurological worsening & $0 / 10(0 \%)$ & & \\
(myelopathy) & & & \\
\hline
\end{tabular}

* Chi square

** Mann-Whitney U

$* * *$ t-test

$* * * *$ Fisher's exact

Table 6: improvement in NDI scores, and VAS scores for arm pain and neck pain at three months and one year after surgery compared to baseline. A significant improvement was present for all outcomes at three months, and at one year. There were no significant differences between the two groups for any of the outcomes at three months and one year after surgery.

\begin{tabular}{lllllll}
\hline & \multicolumn{5}{l}{ Three months after surgery } & \multicolumn{3}{l}{ One year after surgery } \\
\cline { 2 - 6 } & CA & ACD & p-value & CA & ACD & P-value \\
\hline NDI (Mean, SD) & $-23.6(24.1) *$ & $-17.4(16.6) *$ & 0.488 & $-24.4(18.4) *$ & $-24.2(16.1)^{*}$ & 0.982 \\
VAS arm (Mean, $-53.0(39.9) *$ & $-46.7(32.6) *$ & 0.688 & $-61.7(21.2) *$ & $-46.4(30.3) *$ & 0.208 \\
SD) & & & & & \\
VAS neck (Mean, $-37.3(42.7) *$ & $-34.5(34.0) *$ & 0.866 & $-35.5(35.1) *$ & $-35.2(34.1) *$ & 0.988 \\
SD) & & & & & \\
\hline
\end{tabular}

$* \mathrm{P}<0.05$ (compared to baseline, paired t-test)

\section{Adverse events}

There were no superficial or deep wound infections, or any other infections (e.g. urinary tract infection) during hospital stay (Tables 4 and 5). No implant migration or subsidence 
was seen. In the CA group there was one patient who was operated on the C5-C6 level with late-onset ( 6 months after surgery) hypoesthesia and intermittent brachialgia in the left arm corresponding to the $\mathrm{C} 7 / \mathrm{C} 8$ dermatome without pyramidal tract symptoms or pathologic reflexes; MRI did not show nerve root or spinal cord compression, which could be observed well despite some artefacts due to the MRI-compatible implant. In this patient, the complaints spontaneously resolved several weeks later. In the ACD group there were two patients with mild paresis (MRC grade 4) of the deltoid muscle (one ipsilateral to the preoperative complaints, one bilaterally) without sensory deficit, radicular pain, or pathologic reflexes. Additionally, in the ACD group there was one patient with diffuse hypoesthesia in the right arm (ipsilateral to preoperative symptoms), without motor deficit or pathologic reflexes. This patient was also treated for shoulder complaints on that side by an arthroscopic subacromial decompression. There were no patients with myelopathy after surgery.

\section{Secondary radiological outcomes}

\section{Segmental range of motion (sROM)}

At one year, mean sROM in the CA group was $12.3^{\circ}\left(\mathrm{SD} 6.1^{\circ}\right.$ ) versus $3.0^{\circ}\left(\mathrm{SD} 3.6^{\circ}\right)$ in the ACD group (Table 2 ). In the ACD subgroup with preserved motion mean sROM was $7.7^{\circ}$ $\left(\mathrm{SD} 3.7^{\circ}\right)$, while in the ACD subgroup with a fused segment mean sROM was $1.1^{\circ}\left(\mathrm{SD} 0.4^{\circ}\right)$ (Table 2). When compared to baseline, there was no significant difference in sROM at one year at target level for the CA group $\left(12.3^{\circ}\right.$ compared to $\left.11.4^{\circ}, \mathrm{P}=0.526\right)$ and the $A C D$ subgroup with preserved motion $\left(7.7^{\circ}\right.$ compared to $\left.11.7^{\circ}, \mathrm{P}=0.374\right)$. At one year, sROM was significantly lower in the ACD group with a fused segment compared to baseline $\left(1.1^{\circ}\right.$ compared to $\left.15.6^{\circ}, \mathrm{P}=0.002\right)$.

\section{Discussion}

At one year after surgery, the sequence of segmental contributions in C4-C7 that is described in asymptomatic subjects was present in $80 \%$ of the CA group, while in only $20 \%$ of the ACD group (Table 2 and Appendix 1). If normal motion at the operated segment indeed prevents ASDis, this premise is met in the majority of the CA patients.

The amount of patients with a normal sequence of segmental contributions before surgery $(29 \%)$ was higher than we previously found in a smaller subset of this population (10\%) [17]. However, the amount of patients with a normal sequence of segmental contributions is still much lower than the amount that was found in the groups of asymptomatic individuals (85\%) on which the definition of the normal sequence was based [17]. 
Absence of a normal sequence of segmental contributions in the patients preoperatively might be caused by pain. The disturbed sequence of segmental contributions could be analogous to previously described altered patterns in muscle recruitment in patients with chronic neck pain [20]. It could be a way of the body to avoid pain due to radicular compression by narrowing of the neuroforamen during the extension movement, or avoidance of motion in a degenerated disc. However, when comparing the patients with and without a normal sequence of segmental contributions preoperatively, there were no significant differences in VAS score for arm pain $(P=0.579)$, or VAS score for neck pain $(P=0.909)$. Simply the presence of symptoms, not the severity, might be the determining factor. When analyzing differences in SROM at the index level versus the adjacent level within C5-C6 and C6-C7, to see if the diseased segment showed a lower sROM then the adjacent segment within the same patient, the sROM was not significantly different $\left(12.3^{\circ}\right.$ versus $\left.13.0^{\circ}, \mathrm{P}=0.204\right)$.

The difference in the amount of patients with a normal sequence of segmental contributions after surgery between the CA group and ACD group can largely be explained by the high amount of fused segments in the ACD group. Since a patient with a fused segment cannot have a normal sequence of segmental contributions. In the ACD subgroup with preserved motion, 2 out of the 3 patients had a normal sequence of segmental contributions. This was an interesting finding, as the influence of the discectomy on the motion pattern within the motion segment appears to be low (contrary to what was expected). However, given the very low number of patients in this group no conclusion should be drawn on this finding. To be able to show the sequence of segmental contributions in the ACD subgroup with preserved motion a substantially larger sample size for the ACD group would be necessary (about 3 times higher), given the high fusion rate in this group. This is of little clinical relevance because the absolute number of patients with a normal sequence of segmental contributions in the ACD group will be very low. Of the seven patients in the ACD subgroup with a fused segment, the sequence within the two remaining (mobile) segments was normal in relation to each other in five of them.

In total, seven out of the ten patients in the ACD group had a fused segment one year after surgery. This amount is in line with previous studies, which already showed that even without placement of an interposition material to promote fusion, this type of surgery has a high rate of fusion $[4,21]$. Some surgeons see fusion as a main goal of surgery, besides neural decompression, in which case ACDF is preferred to ACD due to the markedly higher fusion rate. Other surgeons see relieving radicular pain as the goal, which is reached equally effective by $A C D$ and $A C D F$, and they see fusion of the segment as a sideeffect of the surgery $[4,21]$. At the time of conception of the study, the latter was the case, and ACD was the standard of practice at the authors' institution. Currently, with an increasing focus on sagittal balance, ACDF is more commonly performed to maintain/restore lordosis at the operated segment. Interestingly, a recently published RCT also questioned the positive influence of CA and ACDF on cervical sagittal balance in the longer term compared to ACD [22]. 
Several subgroup analyses were performed. No significant difference in patient reported outcomes were found when patients with preserved motion at the operated level (both in the CA group and the ACD subgroup with preserved motion) were compared to patients with a fusion. Similarly, no significant differences were found when the patients with preserved motion and a normal sequence were compared to the patients with preserved motion but absence of a normal sequence. The latter might be the results of a type II error, given the high number of fusions.

A downside of the study is the fact that patients and caregivers were not blinded to allocation. Since most outcomes are patient reported this means that these outcomes were unblinded. Prior to participating in the study, all patients were informed that there was no reason to assume better results in the short term for either of the treatments and that a difference in the long term was possible, but all yet unproven. Although adequately informing the patients in this way should limit possible bias, new technology bias is a definite possibility. However, it is highly unlikely that this will change the sequence in which the motion segments in the caudal cervical spine contribute, since the sequence cannot be influenced by voluntary movement.

Attrition rate was $17 \%$ in both treatment groups, this is not expected to be a problem, since the power analysis was calculated with an assumed loss to follow up of $20 \%$, which is why 12 instead of ten patients per arm were included [18].

The duration of inclusion is another downside of the study. Annual inclusion rate was lower than was initially expected. The stringent exclusion criteria, most of all the fact that only soft disc single level pathology without degenerative changes on other levels was included, led to this low rate of inclusion. However, this can also be seen as a strong point of the study as these stringent inclusion criteria lead to less confounding, which would be introduced by varying degrees of degenerative changes in the groups. The influence of varying degrees of degenerative changes on the sequence of segmental contributions is not yet known and should be the focus of further study.

There were more patients in the ACD group with neurological worsening one year after surgery. Of note, none of these patients had new motor or sensory deficit directly after surgery. One of these patients had concomitant shoulder problems on the ipsilateral side, which might have played a role. The two patients with isolated deltoid muscle weakness did not have these complaints directly after surgery, nor at 6 and 12 weeks after surgery. They were in the lower regions of VAS scores for arm pain and neck pain (all between 3 and $20 \mathrm{~mm}$ ), which makes an antalgic paresis unlikely. They did not have a radicular or myelopathic syndrome. Although C5 palsy has been described after ACDF, onset is usually within 10 days after surgery, and is rare when the C4-C5 level is not involved in the decompression [23-25]. The patient in the CA group with clinical symptoms of a more caudal nerve root compression did not show abnormalities on radiographs and on MRI. In this patient, the complaints spontaneously resolved.

All data on the sROMs in the target and adjacent levels is available in Appendix 2. At the target level there was a significant difference in sROM between the two groups at 
one year after surgery, when the patients with a fusion in the ACD group are omitted $(n=3$ remaining), the mean SROM in the ACD group is $7.7^{\circ}$ versus $12.3^{\circ}$ in the prosthesis group $(P=0.246)$. It is thought that fusion of a segment may increase the sROM at the superior and inferior adjacent level, as a compensation for loss of motion [1, 3]. No such increase was found in the ACD group on the superior adjacent level $\left(12.1^{\circ}\right.$ at baseline vs. $13.2^{\circ}$ at one year, $P=0.393)$, or the inferior adjacent level $\left(17.2^{\circ}\right.$ at baseline vs. $15.3^{\circ}$ at one year, $\mathrm{P}=0.532)$. For the $\mathrm{CA}$ group there was no difference on the superior adjacent level $\left(13.3^{\circ}\right.$ at baseline vs. $15.2^{\circ}$ at one year, $\left.\mathrm{P}=0.347\right)$, but a significant decrease at the inferior adjacent level $\left(14.4^{\circ}\right.$ at baseline vs. $11.0^{\circ}$ at one year, $\left.\mathrm{P}=0.019\right)$. The clinical relevance of this decrease is difficult to interpret, as the sROM preoperatively might be influenced by the patients' symptoms at that time and cannot be used as a reference. Usefulness of sROMs in individual patients is already severely impeded by the known high intra- and interindividual variability of sROMs [26]. This variability is further increased by the influence of the method of measurement. In the present study there were differences up to $11^{\circ}$ for a motion segment in the same patient at a single time point, depending on the method of measurement (Appendix 2).

Data on intervertebral translations might also be of importance. These data were also acquired but will be analyzed separately.

The potential clinical relevance of a normal sequence of segmental contributions on itself is doubtful. However, the ultimate goal of CA is prevention of ASDis in the long term. If fusion at the operated segment indeed plays a role in the development of ASDis, preservation of motion with a normal motion pattern may be a prerequisite for prevention of ASDis in the long term. Several studies with longer term follow up (4-7 years) are becoming available, with conflicting results regarding secondary surgery; some studies report a significantly lower rate of secondary surgery for CA than for ACDF, while other studies did not find a significant difference [10-12, 27].

The difference in incidence of ASDis between patients with preservation of motion and a normal sequence of segmental contributions, versus preservation of motion without a normal sequence of segmental contributions is also interesting. This could be used to verify the role of a normal sequence of segmental contributions. Given the number of patients necessary to be able to draw conclusions, it would be beneficial if this method of analysis would be applied more frequently.

The authors aim to plan a long term follow up moment for the population described in this paper, to evaluate the amounts of ASDis, secondary surgery, and long term adverse events, and whether a normal sequence of segmental contributions plays a role in preventing ASDis. 


\section{Conclusion}

At one year after surgery, the sequence of segmental contributions in C4-C7 that is described in asymptomatic subjects was present in $8 / 10$ (80\%) of the CA group, and in 2/10 (20\%) of the ACD group. Fusion rate was $0 / 10(0 \%)$ in the CA group, and $7 / 10(70 \%)$ in the ACD group. There were no differences in patient reported outcomes between the two treatments, nor in any of the subgroup analyses. Therefore the clinical relevance of restoring/preserving a normal motion pattern in the short term is doubtful. However, if fusion at the operated segment indeed plays a role in the development of ASDis, preservation of motion with a normal motion pattern may be a prerequisite for prevention of ASDis in the long term. It would be beneficial if the analysis of the sequence of segmental contributions would be used more commonly, as it is the most consistent parameter for evaluating the presence of physiologic motion in the caudal cervical spine. 


\section{References}

1. Seo, M. and D. Choi, Adjacent segment disease after fusion for cervical spondylosis; myth or reality? $\mathrm{Br} \mathrm{J}$ Neurosurg, 2008. 22(2): p. 195-9.

2. Hilibrand, A.S., et al., Radiculopathy and myelopathy at segments adjacent to the site of a previous anterior cervical arthrodesis. J Bone Joint Surg Am, 1999. 81(4): p. 519-28.

3. Eck, J.C., et al., Biomechanical study on the effect of cervical spine fusion on adjacent-level intradiscal pressure and segmental motion. Spine (Phila Pa 1976), 2002. 27(22): p. 2431-4.

4. Dowd, G.C. and F.P. Wirth, Anterior cervical discectomy: is fusion necessary? J Neurosurg, 1999. 90(1 Suppl): p. 8-12.

5. Heller, J.G., et al., Comparison of BRYAN cervical disc arthroplasty with anterior cervical decompression and fusion: clinical and radiographic results of a randomized, controlled, clinical trial. Spine (Phila Pa 1976), 2009. 34(2): p. 101-7.

6. Mummaneni, P.V., et al., Clinical and radiographic analysis of cervical disc arthroplasty compared with allograft fusion: a randomized controlled clinical trial. J Neurosurg Spine, 2007. 6(3): p. 198-209.

7. Sasso, R.C., et al., Motion analysis of bryan cervical disc arthroplasty versus anterior discectomy and fusion: results from a prospective, randomized, multicenter, clinical trial. J Spinal Disord Tech, 2008. 21(6): p. 3939.

8. Boselie, T.F., et al., Arthroplasty versus fusion in single-level cervical degenerative disc disease: a Cochrane review. Spine (Phila Pa 1976), 2013. 38(17): p. E1096-107.

9. Donk, R.D., et al., What's the best surgical treatment for patients with cervical radiculopathy due to singlelevel degenerative disease? A randomized controlled trial. PLoS One, 2017. 12(8): p. e0183603.

10. Hisey, M.S., et al., Prospective, Randomized Comparison of Cervical Total Disk Replacement Versus Anterior Cervical Fusion: Results at 48 Months Follow-up. J Spinal Disord Tech, 2015. 28(4): p. E237-43.

11. Janssen, M.E., et al., ProDisc-C Total Disc Replacement Versus Anterior Cervical Discectomy and Fusion for Single-Level Symptomatic Cervical Disc Disease: Seven-Year Follow-up of the Prospective Randomized U.S. Food and Drug Administration Investigational Device Exemption Study. J Bone Joint Surg Am, 2015. 97(21): p. 1738-47.

12. Sasso, R.C., et al., Results of cervical arthroplasty compared with anterior discectomy and fusion: four-year clinical outcomes in a prospective, randomized controlled trial. J Bone Joint Surg Am, 2011. 93(18): p. 1684-92.

13. Burkus, J.K., et al., Long-term clinical and radiographic outcomes of cervical disc replacement with the Prestige disc: results from a prospective randomized controlled clinical trial. J Neurosurg Spine, 2010. 13(3): p. 308-18.

14. Phillips, F.M., et al., Long-term Outcomes of the US FDA IDE Prospective, Randomized Controlled Clinical Trial Comparing PCM Cervical Disc Arthroplasty With Anterior Cervical Discectomy and Fusion. Spine (Phila Pa 1976), 2015. 40(10): p. 674-83.

15. Bogduk, N. and S. Mercer, Biomechanics of the cervical spine. I: Normal kinematics. Clin Biomech (Bristol, Avon), 2000. 15(9): p. 633-48.

16. Van Mameren, H., Motion patterns in the cervical spine. 1988, Maastricht University.

17. Boselie, T.F., et al., A Pilot Study Of Sequence Of Segmental Contributions In The Lower Cervical Spine During Active Extension And Flexion: Healthy Controls vs. Cervical Degenerative Disc Disease Patients. Spine (Phila Pa 1976), 2016.

18. Boselie, T.F., et al., Cervical spine kinematics after anterior cervical discectomy with or without implantation of a mobile cervical disc prosthesis; an RCT. BMC Musculoskelet Disord, 2015. 16: p. 34.

19. Reinartz, R., et al., Cervical vertebrae tracking in video-fluoroscopy using the normalized gradient field. Med Image Comput Comput Assist Interv, 2009. 12(Pt 1): p. 524-31.

20. Tsang, S.M., G.P. Szeto, and R.Y. Lee, Altered spinal kinematics and muscle recruitment pattern of the cervical and thoracic spine in people with chronic neck pain during functional task. J Electromyogr Kinesiol, 2014. 24(1): p. 104-13. 
21. Wirth, F.P., et al., Cervical discectomy. A prospective analysis of three operative techniques. Surg Neurol, 2000. 53(4): p. 340-6; discussion 346-8.

22. Donk, R.D., et al., Cervical sagittal alignment after different anterior discectomy procedures for single-level cervical degenerative disc disease: randomized controlled trial. Acta Neurochir (Wien), 2017.

23. Kim, S., et al., Clinical and radiographic analysis of c 5 palsy after anterior cervical decompression and fusion for cervical degenerative disease. J Spinal Disord Tech, 2014. 27(8): p. 436-41.

24. Yang, C.W. and J.L. Fuh, C5 palsy after cervical spine decompression surgery. J Chin Med Assoc, 2013. 76(7): p. 363-4.

25. Wang, $\mathrm{H}$., et al., Analysis of correlative risk factors for $\mathrm{C} 5$ palsy after anterior cervical decompression and fusion. Int J Clin Exp Med, 2015. 8(3): p. 3983-91.

26. Van Mameren, $H_{\text {., }}$ et al., Cervical spine motion in the sagittal plane (I) range of motion of actually performed movements, an X-ray cinematographic study. Eur J Morphol, 1990. 28(1): p. 47-68.

27. Boselie, T.F. and H. van Santbrink, Arthroplasty in cervical degenerative disc disease: fulfilling its long-term promise? J Spine Surg, 2016. 2(4): p. 359-361. 


\section{Appendices}

\section{Chapter 6 - Appendix 1 - Graphs depicting sequence of segmental contributions ('sequence') within block C4-C7 before surgery and 1 year after surgery.}

All the graphs depicting the sequence of segmental contributions by segments C4-C5, C5-C6 and C6-C7 within block C4-C7 are visible in the next four pages. Presence or absence of a normal sequence was scored based on the definition of the normal sequence, which was based on healthy controls.

Patients 1-3 were part of a pilot to limit a possible learning curve and are not depicted. Patients 4-27 are divided into four groups, based on the allocated treatment and the level that was operated. The graphs from each of these patients before surgery and 1 year after surgery are presented as pairs.

${ }^{1}$ Boselie TF, van Santbrink H, de Bie RA, van Mameren H. A Pilot Study Of Sequence Of Segmental Contributions In The Lower Cervical Spine During Active Extension And Flexion: Healthy Controls vs. Cervical Degenerative Disc Disease Patients. Spine (Phila Pa 1976). 2016 


\section{Disc prosthesis group, only patients operated on C5-C6}

\section{Patient No}

4

Rotation between successive

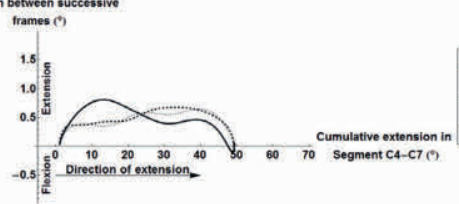

Preoperative

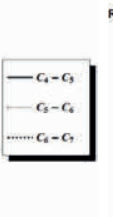

1 year postoperative

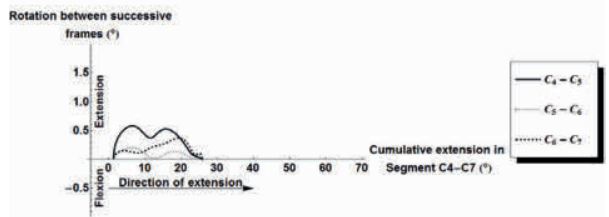

Patient 4 did not have a normal sequence before surgery (the peak in $\mathrm{C} 6-\mathrm{C} 7$ precedes the peak in $\mathrm{C} 5-\mathrm{C} 6$ at the end of extension). 1 year after surgery the sequence also was not normal (the peak in C5-C6 is below 0.3 degrees between successive frames, the sequence in the other two segments is correct).

5

Rotation between euccesove

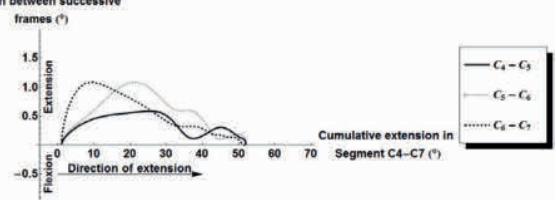

Patient 5 did not have a normal sequence before surgery (the sequence ends with a peak in C4-C5), and was not available for follow up 1 year after surgery.

13

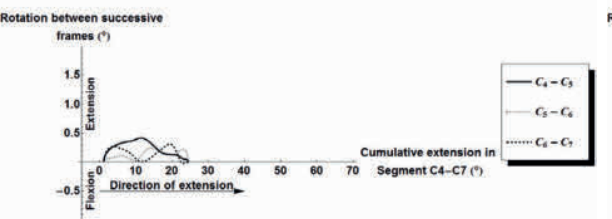

Rotation between successive

frames ${ }^{\circ}$

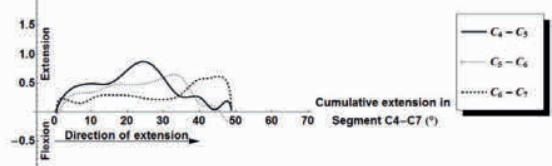

Patient 13 did not have a normal sequence before surgery (no peak above $0.3^{\circ}$ rotation in $\mathrm{C} 5-\mathrm{C} 6$ after the peak in C4-C5), while the sequence was normal 1 year after surgery.

15

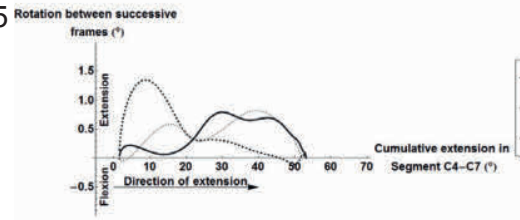

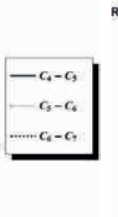

Rotation betreen successive

frames (9)

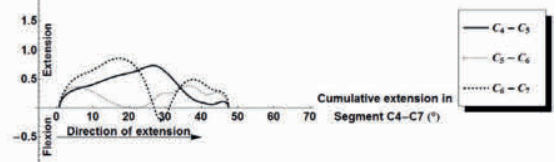

Patient 15 did not have a normal sequence before surgery (no peak in $\mathrm{C} 6-\mathrm{C} 7$ at the end of extension), while the sequence was normal 1 year after surgery (the small peaks in $\mathrm{C} 5-\mathrm{C} 6$ and $\mathrm{C} 6-\mathrm{C} 7$ at the end are below $0.3^{\circ}$ rotation).

17 R

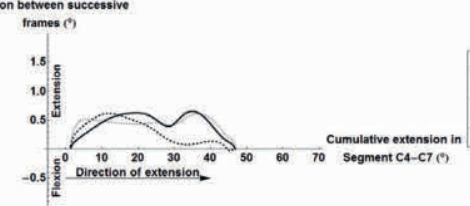

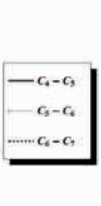

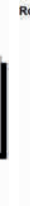

Patient 17 did not have a normal sequence before surgery (no peak in $\mathrm{C6}-\mathrm{C} 7$ at the end of extension), while the sequence was normal 1 year after surgery. 
$\underline{\text { Patient No }}$

21 Rotation between successive

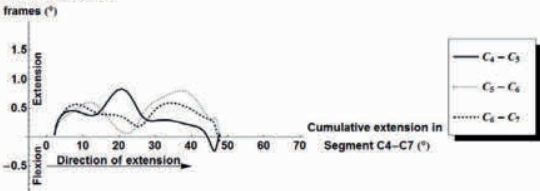

Patient 21 did not have a normal sequence before surgery (the peak in C6-C7 precedes the peak in C5-C6), and was not available for follow up 1 year after surgery.

22

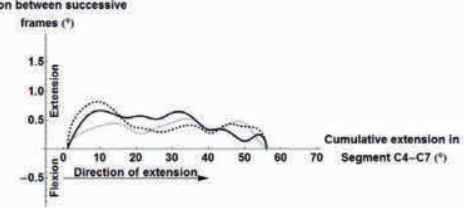

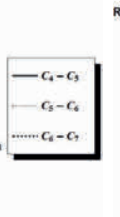

Rotation between successive

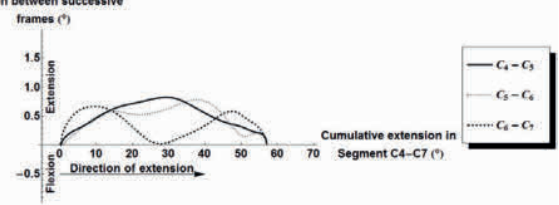

Patient 22 did not have a normal sequence before surgery (concluding peak in C5-C6), while there was a normal sequence 1 year after surgery.

23

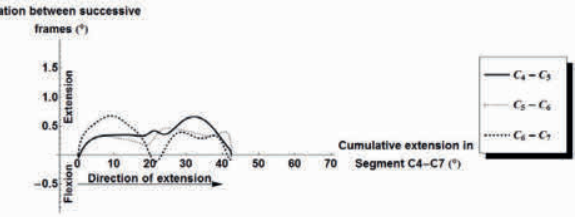

Rotation between successive

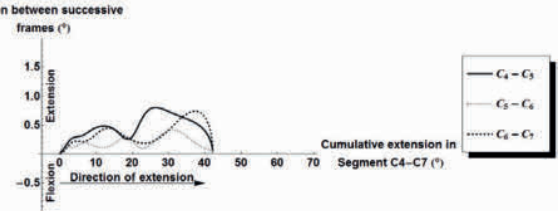

Patient 23 did not have a normal sequence before surgery (concluding peak in C5-C6), while the sequence was normal 1 year after surgery.

24 Rotation between successive

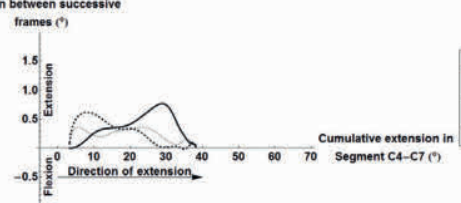

Rotation between successive

frames o
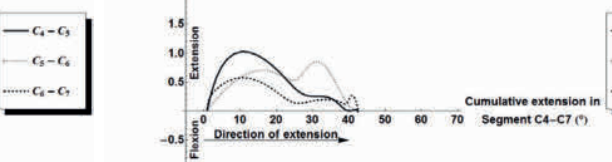

Patient 24 did not have a normal sequence before surgery (concluding peak in C4-C5), while the sequence still was not normal 1 year after surgery (no concluding peak in C6-C7). 
Disc prosthesis group, only patients operated on C6-C7

Patient No

9 Rotation between successive

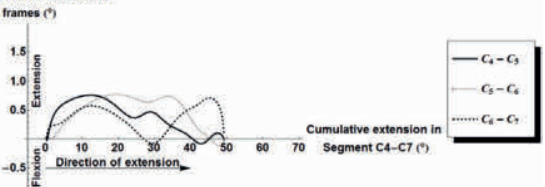

Preoperative

tion between successive

$$
\text { frames }{ }^{\circ}
$$

\section{1 year postoperative}

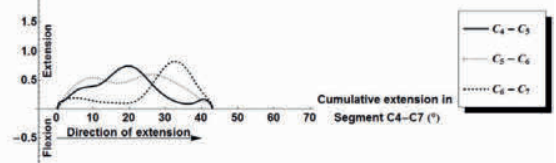

Patient 9 had a normal sequence before surgery, and also a normal sequence 1 year after surgery.

12

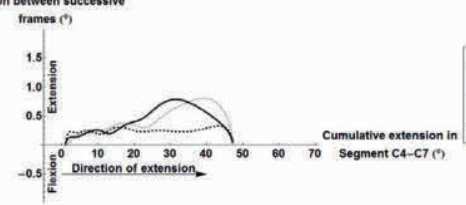

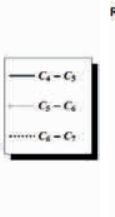

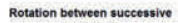

$$
\begin{aligned}
& \text { frames } \\
& \text { (⿻) }
\end{aligned}
$$

frames (c)

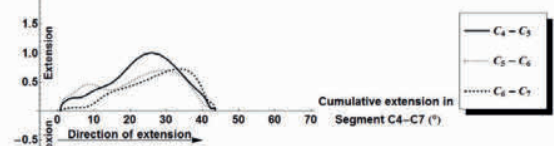

$\frac{8}{4}$

Patient 12 had a normal sequence before surgery (the peak in C6-C7 at the end is above $0.3^{\circ}$ rotation), and also a normal sequence 1 year after surgery.
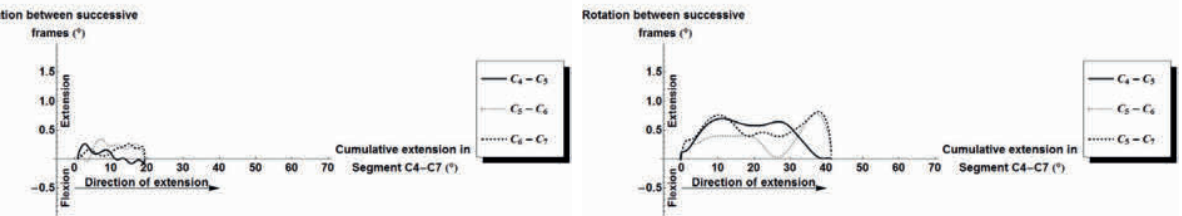

Patient 27 did not have a normal sequence before surgery (almost absent motion in all three segments), while the sequence was normal 1 year after surgery. 


\section{ACD group, only patients operated on C5-C6}

\section{Patient No}

6 Rotation between successive

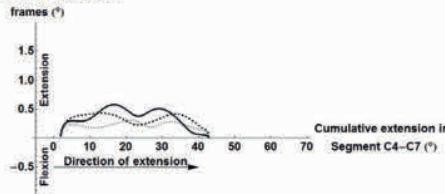

\section{1 year postoperative}

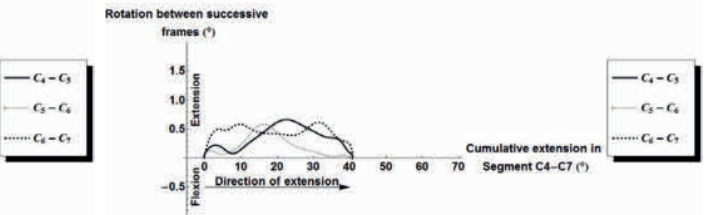

Patient 6 had a normal sequence before surgery, while 1 year after surgery it was not normal (the peak in C5-C6 precedes the peak in C4-C5).

8

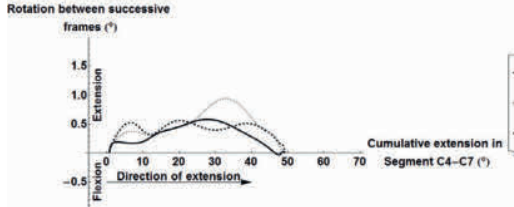

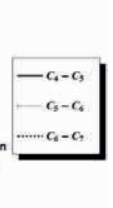

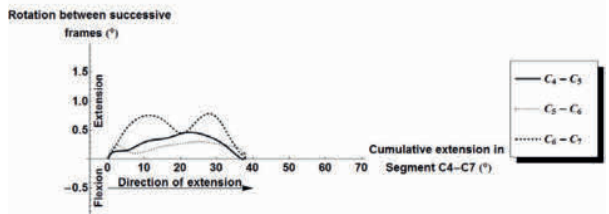

Patient 8 had a normal sequence before surgery, and also a normal sequence 1 year after surgery (the peak in C5-C6 is just above $0.3^{\circ}$ rotation between successive frames).

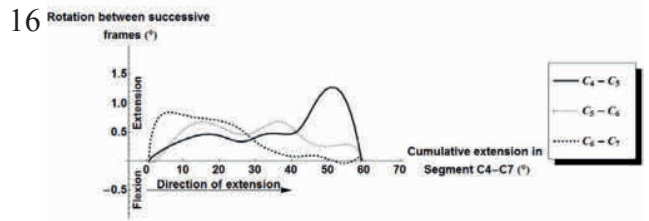

Patient 16 did not have a normal sequence before surgery (no peak in C6-C7 at the end of extension), and was not available for follow up 1 year after surgery.

19 Rotation between successive

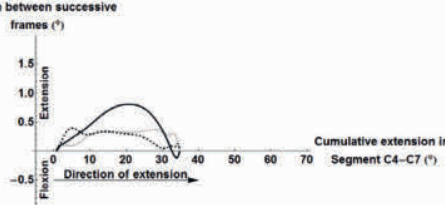

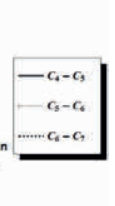

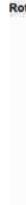

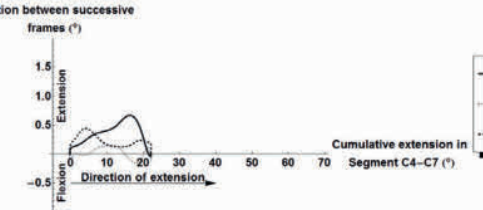

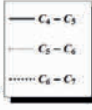

Patient 19 did not have normal sequence before surgery (no concluding peak in C6-C7), and also did not have a normal sequence 1 year after surgery (not only due to absence of a peak $>0.3^{\circ}$ rotation in $\mathrm{C} 5-\mathrm{C} 6$, but also since there is no concluding peak in C6-C7)

20 Rotation between successive frames $(0)$

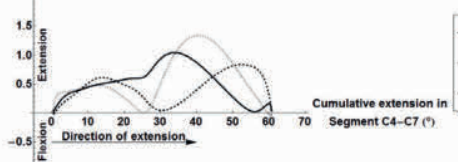

Patient 20 had a normal sequence before surgery, while the sequence 1 year after surgery was not normal (due to fusion in C5C6, the sequence in the two remaining segments is correct). 
26 Rotation between successive

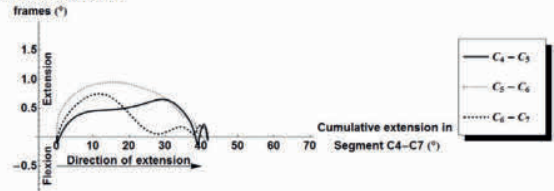

Patient 26 did not have a normal sequence before surgery (C4-C5 after C5-C6 and no concluding peak in C6-C7), and was not available for follow up 1 year after surgery. 
ACD group, only patients operated on C6-C7

Patient No

7 Rotation between successive

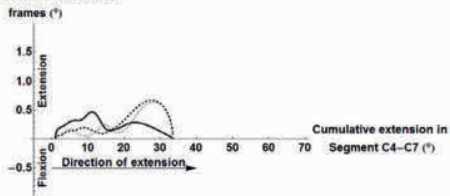

Preoperative

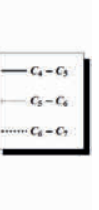

1 year postoperative

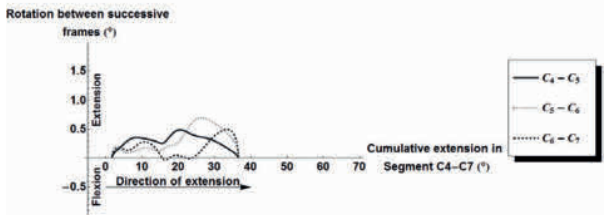

Patient 7 did not have a normal sequence before surgery (peak in C6-C7 slightly preceeding C5-C6), while 1 year after surgery the sequence was normal.
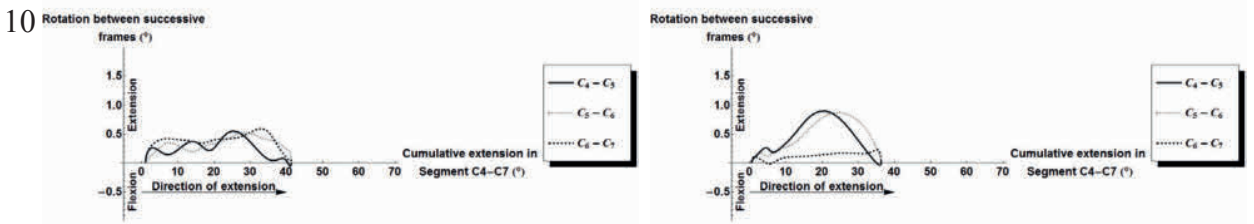

Patient 10 had a normal sequence before surgery, while the sequence was not normal 1 year after surgery (due to fusion in C5$\mathrm{C6}$, the sequence within the remaining motion segments is correct).

11

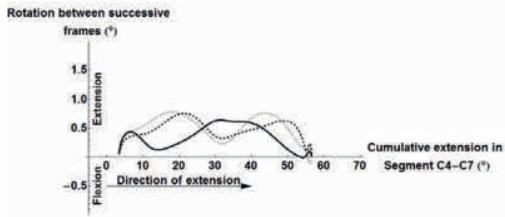

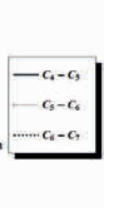

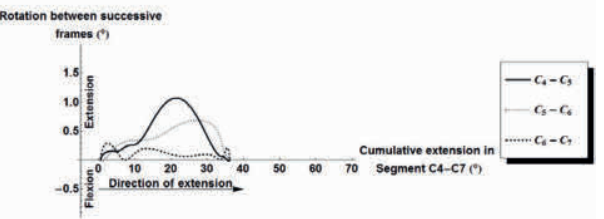

Patient 11 had a normal sequence before surgery, while the sequence was not normal 1 year after surgery (based on (near) fusion in the operated segment, the sequence within the remaining motion segments is correct).

14

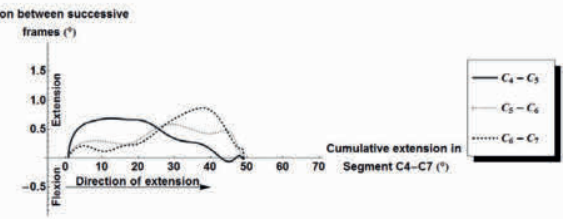

Rotation between successive

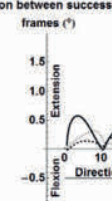

Patient 14 did not have a normal sequence before surgery (peak in C5-C6 after the peak in C6-C7), and still was not normal 1 year after surgery (due to fusion in the operated segment, the sequence in the two remaining segments is correct).

18 R

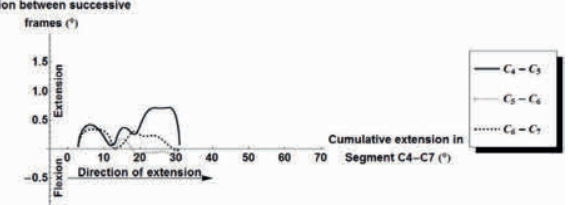

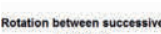

$$
\begin{array}{r}
1.5 \\
1.0 \\
0.5 \\
-0.5
\end{array}
$$

Patient 18 did not have a normal sequence before surgery (peak in C4-C5 at the end of extension), the sequence still was not normal 1 year after surgery (due to fusion in the operated segment, the sequence in the two remaining segments is correct). 
$\underline{\text { Patient No }}$

25 Rotation between successive

trames (9)

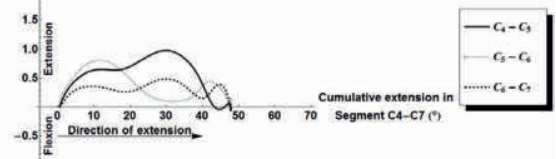

Preoperative
1 year postoperative

Rotation between successive

trames (o)

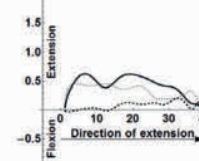

0.5
Cumulative extension in $\begin{array}{r}-c_{\mathrm{s}}-c_{9} \\ -c_{\mathrm{s}}-c_{\mathrm{c}} \\ \cdots \cdots \\ \cdots\end{array}$

Segment $\mathrm{C} 4-\mathrm{C} 7 \mathrm{C}$ ?

Patient 25 did not have a normal sequence before surgery (peak in C6-C7 just after peak in C4-C5 and before the peak in C5C6), the sequence still was not normal 1 year after surgery (due to fusion in the operated segment). 
Chapter 6 - Appendix 2 - sROMs at target and adjacent levels Table A - Disc prosthesis, only patients operated on C5-C6

\begin{tabular}{|c|c|c|c|c|c|c|c|c|}
\hline \multirow[t]{2}{*}{ Pat no. } & \multicolumn{2}{|c|}{ Sequence } & \multirow[t]{2}{*}{ Level } & \multirow{2}{*}{$\begin{array}{c}\text { Preop } \\
\text { sROM F } \rightarrow E\end{array}$} & \multirow{2}{*}{$\begin{array}{c}\text { Preop } \\
\text { sROM E } \rightarrow \text { F }\end{array}$} & \multirow{2}{*}{$\begin{array}{l}\text { Post-op } 1 \text { yr } \\
\text { sROM F } \rightarrow E\end{array}$} & \multirow{2}{*}{$\begin{array}{l}\text { Post-op } 1 \text { yr } \\
\text { sROM E } \rightarrow F\end{array}$} & \multirow[t]{2}{*}{$\Delta$ sROM (1 yr - preop) } \\
\hline & Preoperative & $1 \mathrm{yr}$ & & & & & & \\
\hline \multirow{3}{*}{4} & \multirow{3}{*}{ Not normal } & \multirow{3}{*}{ Not normal } & $\mathrm{c} 4 \mathrm{c5}$ & 17.0 & 19.3 & 12.0 & 9.0 & -7.7 \\
\hline & & & c5c6 & 10.3 & 13.7 & 5.7 & 5.3 & -6.5 \\
\hline & & & $\mathrm{c} 6 \mathrm{c} 7$ & 15.3 & n.v. * & 9.3 & 7 & -7.2 \\
\hline \multirow{3}{*}{5} & \multirow{3}{*}{ Not normal } & \multirow{3}{*}{ n.a. ** } & $\mathrm{c} 4 \mathrm{c5}$ & 10.7 & 10.0 & n.a. ** & n.a. ** & n.a. ** \\
\hline & & & c5c6 & 14.7 & 15.7 & n.a. ** & n.a. ** & n.a. ** \\
\hline & & & $\mathrm{c} 6 \mathrm{c} 7$ & 22.7 & 14.7 & n.a. ** & n.a. ** & n.a. ** \\
\hline \multirow{3}{*}{13} & \multirow{3}{*}{ Not normal } & \multirow{3}{*}{ Normal } & $\mathrm{c} 4 \mathrm{c5}$ & 10.0 & 10.0 & 19.0 & 16.0 & 7.5 \\
\hline & & & c5c6 & 2.3 & 8.0 & 19.0 & 18.7 & 13.7 \\
\hline & & & $\mathrm{c} 6 \mathrm{c} 7$ & 9.7 & n.v. * & 5.3 & 16.7 & 1.3 \\
\hline \multirow{3}{*}{15} & \multirow{3}{*}{ Not normal } & \multirow{3}{*}{ Normal } & $\mathrm{c} 4 \mathrm{c5}$ & 19.0 & 15.3 & 14.3 & 18.3 & -0.8 \\
\hline & & & c5c6 & 11.3 & 13.0 & 7.7 & 6.3 & -5.2 \\
\hline & & & $\mathrm{c} 6 \mathrm{c} 7$ & 19.7 & 9.7 & 19.0 & 14.7 & 2.2 \\
\hline \multirow{3}{*}{17} & \multirow{3}{*}{ Not normal } & \multirow{3}{*}{ Normal } & $c 4 c 5$ & 11.3 & 15.0 & 17.7 & 14.7 & 3.0 \\
\hline & & & c5c6 & 18.0 & 13.3 & 22.0 & 17.3 & 4.0 \\
\hline & & & $\mathrm{c} 6 \mathrm{c} 7$ & 12.7 & 15.7 & 10.0 & n.v. * & -4.2 \\
\hline \multirow{3}{*}{21} & & & $\mathrm{c} 4 \mathrm{c5}$ & 12.7 & 12.7 & n.a. ** & n.a. ** & n.a. ** \\
\hline & Not normal & n.a. ** & c5c6 & 13.7 & 18.0 & n.a. ** & n.a. ** & n.a. ** \\
\hline & & & $\mathrm{c} 6 \mathrm{c} 7$ & 15.7 & n.v. * & n.a. ** & n.a. ** & n.a. ** \\
\hline & & & $\mathrm{c} 4 \mathrm{c5}$ & 18.0 & 15.0 & 19.3 & 12.7 & -0.5 \\
\hline 22 & Not normal & Normal & c5c6 & 12.7 & 11.3 & 18.0 & 14.3 & 4.2 \\
\hline & & & $\mathrm{c} 6 \mathrm{c} 7$ & 19.3 & 14.0 & 15.0 & 15.3 & -1.5 \\
\hline & & & $\mathrm{c} 4 \mathrm{c} 5$ & 14.0 & 12.0 & 15.0 & 15.7 & 2.3 \\
\hline 23 & Not normal & Normal & $\mathrm{c} 5 \mathrm{c} 6$ & 10.3 & 7.3 & 6.0 & 5.7 & -3.0 \\
\hline & & & $\mathrm{c} 6 \mathrm{c7}$ & 11.3 & 12.7 & 12.3 & 9 & -1.3 \\
\hline & & & c4c5 & 13.7 & 13.7 & 9.3 & 13.7 & -2.2 \\
\hline 24 & Not normal & Not normal & $c 5 c 6$ & 10.0 & 9.3 & 14.0 & 22.0 & 8.3 \\
\hline & & & $\mathrm{c} 6 \mathrm{c} 7$ & 13.0 & 7.3 & 6.0 & n.v. * & -4.2 \\
\hline Disc pro & sthesis, only $\mathrm{p}$ & atients oper & $\mathrm{C} 6-\mathrm{C} 7$ & & & & & \\
\hline Pat no. & Sequ & nce & Level & Preop & Preop & Post-op 1 yr & Post-op 1 yr & $\Delta$ sROM (1 yr - preop) \\
\hline & Preoperative & $1 \mathrm{yr}$ & & $\mathrm{sROM} F \rightarrow E$ & $\mathrm{sROM} \mathrm{E} \rightarrow \mathrm{F}$ & $\mathrm{sROM} F \rightarrow E$ & sROM E $\rightarrow F$ & \\
\hline & & & $c 5 c 6$ & 15.0 & 15.0 & 15.7 & 14.0 & -0.2 \\
\hline 9 & Normal & Normal & $\mathrm{c} 6 \mathrm{c} 7$ & 14.3 & 13.0 & 15.7 & 9.7 & -1.0 \\
\hline & & & $\mathrm{c} 7 \mathrm{t} 1$ & n.v. * & n.v. * & 11.7 & 15 & n.a. ** \\
\hline & & & c5c6 & 13.7 & 12.0 & 15.7 & 13.0 & 1.5 \\
\hline 12 & Normal & Normal & $\mathrm{c} 6 \mathrm{c} 7$ & 16.0 & 12.0 & 8.3 & 7.3 & -6.2 \\
\hline & & & $\mathrm{c} 7 \mathrm{t} 1$ & n.v. * & 8.3 & n.v. * & n.v. * & n.a. ** \\
\hline & & & c5c6 & 1.7 & 2.3 & 13.7 & 5.0 & 7.3 \\
\hline 27 & Not normal & Normal & $\mathrm{c} 6 \mathrm{c} 7$ & 3.0 & 2.3 & 6.7 & 4.7 & 3.0 \\
\hline & & & $\mathrm{c} 7 \mathrm{t} 1$ & n.v. * & n.v. * & n.v. * & n.v. * & n.a. ** \\
\hline
\end{tabular}




\section{Chapter 6 - Appendix 2 - sROMs at target and adjacent levels}

Table B: patients with an anterior cervical discectomy (ACD) without placement of a motion preserving disc prosthesis. Six of these patients were operated on the C5-C6 level, and six of them on C6-C7. Five of them had a normal sequence of segmental contributions before surgery, while two out of ten had a normal sequence of segmental contributions one year after surgery (out of the eight patients without a normal sequence this was solely due to the fusion in six, the other two segments had a correct sequence). Two patients were lost to follow-up. In seven of the patients the operated level was fused $\left(\mathrm{sROM}<2^{\circ}\right)$ one year after surgery. On the cranial adjacent level there was no significant change in SROM, there was no consistent increase or decrease in SROM. No conclusion can be made from either an increase or decrease in SROM, as it is compared to an abnormal reference (the patients are symptomatic before surgery). Of note, there were large differences between sROMs measured on the Flexion to Extension $(\mathrm{F} \rightarrow \mathrm{E})$ movement, and sROMs measured on the Extension to Flexion $(\mathrm{E} \rightarrow \mathrm{F})$ movement (up to $11.3^{\circ}$ for a segment in the same patient at the same moment).

The only conclusion that can be made using SROM data is the fact that there is presence or absence of motion at the operated segment. The outcome parameter is too variable to be useful for conclusions about normal or abnormal motion at the operated level, or adjacent levels.

Table B - ACD, only patients operated on C5-C6

\begin{tabular}{|c|c|c|}
\hline \multirow[t]{2}{*}{ Pat no. } & \multicolumn{2}{|c|}{ Sequence } \\
\hline & Preoperative & $1 \mathrm{yr}$ \\
\hline 6 & Normal & Not normal \\
\hline 8 & Normal & Normal \\
\hline 16 & Not normal & n.a. ** \\
\hline 19 & Not normal & Not normal \\
\hline 20 & Normal & Not normal \\
\hline 26 & Not normal & n.a. ${ }^{* *}$ \\
\hline
\end{tabular}

Level

c4c5

$\mathrm{c} 5 \mathrm{c} 6$
$\mathrm{c} 6 \mathrm{c} 7$

405

c5 6

c6c7

c4c5

c5c6

c6c7

c4c5

c5c6
c6c7

c4c5

c5c6

c4c5

c5c6
c6c7
Preop
ROM F $\rightarrow$ Preop
sROM

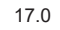

9.3

18.7

14.0

17.0

14.3

18.3

18.7

17.3

10.3

9.7

12.3

15.0

19.7

23.3

10.0

13.7

14.0
Level

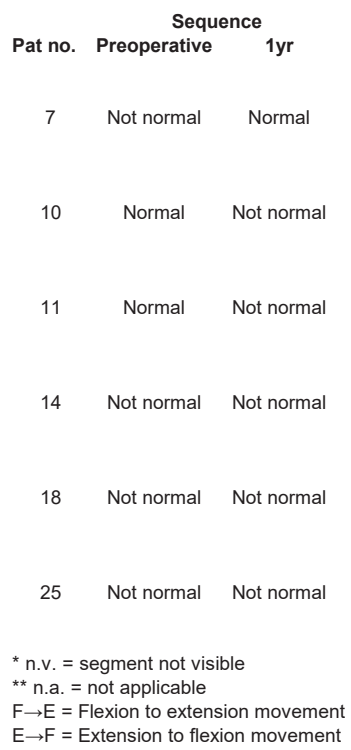

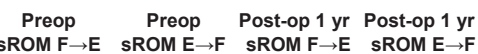

55

c6c7

c7t1

c5c6

$\mathrm{c} 6 \mathrm{c} 7$

$\mathrm{c} 7 \mathrm{t} 1$

c5 56

$c 5 c 6$
$c 6 c 7$

$\mathrm{c} 7 \mathrm{t} 1$

c5 6

c6c7

c7t1

c5 6

$\mathrm{c} 5 \mathrm{c} 6$
$\mathrm{c} 6 \mathrm{c} 7$

c7t1

c5 6

c6c7

c7t1

8.7

8.7

n.v. *

6.7
11.3

\section{3}

11.3

6.0

n.v.

14.7

$\begin{array}{cc}1.3 & 1.7 \\ \text { n.v. } & \end{array}$

12.7

12.7
1.7

n.v. *

7.3

1.3

8

8.0
1.0

n.v. *

8.0

1.7
n.v.
$\Delta$ sROM (1 yr - preop)

3.5

$-4.5$

2.5

$-2.5$

$-7.5$

2.0

n.a. **

n.a. **

n.a. **

4.5

$-7.8$

$-5.5$

$-4.3$

$-4.2$

n.a. **

n.a. **

n.a. **

$\Delta$ sROM (1 yr - preop)

3.0

n.a. **

7.0

$-9.5$

n.a. **

$-3.5$

$-17.8$

n.a. **

0.3

$-13.5$

n.a. **

$-2.0$

$-5.5$

n.a. **

$-2.2$

$-12.7$ 

Chapter

General discussion 
The main goal of this thesis was to determine whether cervical arthroplasty restores normal motion in cervical degenerative disc disease (CDDD) patients. As we discussed in Chapter 1 of this thesis, preservation of motion in the operated segment by cervical arthroplasty is intended to reduce the incidence of adjacent segment disease (ASDis) after anterior cervical discectomy. Anterior cervical discectomy (ACD) can be performed with or without fusion-promoting actions and is referred to as ACDF and ACD, respectively. As ASDis is a long term phenomenon after $A C D(F)$, the assumed beneficial effect of cervical arthroplasty cannot be determined on the basis of outcomes of short term studies. At the time of conception of the RCT as described in this thesis, no long term data were available. Even to date there is a paucity of good quality long term data, these studies are only just beginning to be reported [1-5].

To determine whether short term results were sufficiently similar to justify an RCT that is aimed at motion analysis, i.e. to determine if the principle of clinical equipoise was met, a systematic review and meta-analysis were performed on the available studies. The results of this systematic review are described in Chapter 2. It showed that results for most of the outcome parameters were significantly better for arthroplasty than for fusion. However, the difference in effect size was not clinically relevant for any of these outcomes, with the exception of the amount of segmental range of motion, which was much higher in the arthroplasty group. The latter finding was expected, as one of the treatments is aimed at fusing the operated segment, while the other aims at preserving motion in the operated segment. Based on this review we concluded that cervical arthroplasty can be performed in studies, as an alternative for the commonly performed fusion, while awaiting long term results.

A second issue was the lack of an adequate definition of normal motion and the way to assess it. The conventional method of motion analysis, determining segmental range of motions (sROMs) is known for its high intra- and interindividual variability, which makes it useless to determine normal motion in individual patients. An alternative was a more dynamic method of analysis, enabling the description of motion patterns. This method was based on historic data, in which motion patterns in the cervical spine were described. These patterns were reported to be consistent within and between subjects. As this method of analysis is very time consuming due to the number of vertebrae that need to be tracked, and the number of frames in a single recording, we aimed to develop a computer assisted or automated method of image analysis. In conjunction with the department of Biomedical Engineering from the Eindhoven University of Technology an image analysis algorithm was developed that was able to track the skull base and all cervical vertebrae throughout all frames of a recording of a subject performing a full bending flexion to extension movement (and vice versa). This image recognition algorithm, which uses normalized gradient field analysis, is described in Chapter 3.

This tracking algorithm was then used on flexion and extension cinematographic recordings (FCRs/ECRs) of two groups of ten asymptomatic subjects (healthy controls). A consistent motion pattern was identified, being present in the vast majority of healthy 
controls, often at both time points at which the recordings were made. A definition of normal motion was created. To test sensitivity and specificity of the definition in differentiating between healthy controls and pre-operative CDDD patients, a panel of independent raters was asked to score a series of graphs depicting these motion patterns at three time points. Intra- and interrater agreement were high (average kappa of 0.85 and Fleiss' kappa of 0.82 , respectively). Sensitivity and specificity were very high, $90 \%$ and $85 \%$, respectively. In contrast it was shown that it was not possible to differentiate between healthy controls and pre-operative CDDD patients by using sROMs. These findings are described in Chapter 4. The definition that was described in Chapter 4 was then applied to what it was developed for, and used as a primary outcome measure in an RCT comparing cervical arthroplasty (CA) and ACD. Details of this RCT are described in Chapter 5. It was then shown that at one year after having undergone CA, 8 out of 10 patients had a normal motion pattern. In the ACD group this was only 2 out of 10 patients, most likely due to the high rate of fusion. This is described in Chapter 6.

Therefore, the primary aim of this thesis was reached; a definition of normal motion was determined, and this definition was applied to post-operative CDDD patients that had undergone CA.

The secondary aim was to compare (patient reported) outcomes for CA and for ACD. No clinically relevant differences were seen between the two groups, apart from a higher amount of patients with intact mobility of the operated segment in the CA group one year after surgery. This is also described in Chapter 6.

\section{Origin of the motion pattern: some reflections}

The primary focus of this thesis was to investigate if cervical arthroplasty restores/preserves normal motion in the sagittal plane after anterior cervical discectomy for CDDD. Although a consistent motion pattern was found in healthy controls and was used to create a definition for normal motion, this sheds no light on the origin of the motion pattern itself. Understanding why a certain pattern is found can help in the interpretation of its relevance. The reason why this specific sequence of segmental contributions is found is most likely because this is the way to move the cervical spine with the least effort when sitting in an upright position. This means movement with maximal 'help' of gravity and with as little motion against the vector of gravity as possible. In this case it is probably most efficient to move the heads' centre of gravity dorsally early in the movement, as this will aid in extension of the cervical spine.

The reason why this motion pattern is not present in the majority of our population before surgery is possibly of antalgic origin. Stretching of a nerve root during flexion, or compression on the nerve root due to foraminal narrowing during extension might be causing pain. This could lead to avoidance of certain movements. When target level sROM preoperative and at one year postoperative in de CA group were compared this did not 
reflect in a significant increase in target level SROM (10.8 (SD: 5.05) versus $12.3^{\circ}$ (SD: 6.1), respectively, $P=0.519)$. Nor was there a significant increase in sROM block C4-C7 for this group (41.29 (SD: 12.1 ) versus $44.1^{\circ}$ (SD: 9.0), respectively, $\left.\mathrm{P}=0.549\right)$. This indicates that the presence of antalgia did not result in a substantial decrease in SROM. This could be due to the researcher motivating the patients during the fluoroscopic recordings to move as far as possible, through the pain. It therefore does not exclude the possibility that the painful part of the motion is delayed, rather than not performed at all. Recently, the radiological data at three months after surgery have been analysed (data not yet published). Interestingly, only 2 out of 11 patients in the CA group had a normal sequence of segmental contributions at this time point, while this was 8 out of 10 patients at one year after surgery (see Chapter 6). There was no significant difference in VAS scores for arm and neck pain, or for any of the patient reported outcomes between the three month and one year time point. This makes residual nerve root compression at three months as a cause at this time point unlikely. The cervical disc prosthesis is the same at both time points, so should not be an influence as well. Scar tissue due to the surgery is a possibility, but this should still be present at one year after surgery. Maturation of the scar tissue could allow for more mobility. A possible explanation is an altered motion pattern due to an antalgic pattern of muscle recruitment. A relation between pain and coordination of muscle activity (called the pain-adaptation model) during movement has previously been demonstrated $[6,7]$. In patients with chronic neck pain, altered patterns in muscle recruitment to avoid pain have also been described [8]. This might also be the case in patients without a normal sequence of segmental contributions. Although pain had significantly decreased at the first time point of follow up, it is possible that they maintained an antalgic muscle recruitment pattern for some time before returning to a more normal pattern, leading to return of the normal sequence of segmental contributions. Persisting motor adaptation despite resolution of pain has been described in patients with recurrent episodes of pain $[9,10]$. The most recent episode of pain in the subjects in those publications was always within the past three months, it is therefore not possible to state how long signs of persistent motor adaptation remain present. Additionally, a persisting antalgic standing position was described in patients one month after decompression surgery for lumbar spinal stenosis, although spinal movement during gait approximated normal movement at that same time point [11]. As with the previous two publications the follow up was limited, in this case never longer than one month after surgery.

Interestingly, two of the ACD patients had a normal motion pattern at one year after surgery, while the contents of the disc space as well as the cartilage endplates are removed during surgery. This might indicate that the design of a disc prosthesis is of limited influence, and that the pattern is steered by other factors such as the facet joints and surrounding soft tissues. 


\section{Role of this method of motion analysis in the treatment of CDDD}

Using the analysis of sequence of segmental contributions (SOSC analysis) one can determine whether a patient not only has preserved motion at one year after surgery, but also if motion is restored/preserved with a physiological motion pattern.

In the current study we found no difference in outcome between CA and ACD, this was also the case in the subgroup analysis of patients with a normal motion pattern compared to those without a normal motion pattern. Therefore, the clinical relevance of a 'normal motion pattern' is doubtful at one year after surgery.

If the premise that normal motion decreases the risk of developing ASDis is correct, a correlation can be found between a normal sequence of segmental contributions and a lower risk of ASDeg/ASDis on the long term in future studies. This would make this method a very useful tool in the follow up of CA. It would then be possible to evaluate future effects of an implant design relatively quickly, without the need for long term studies on this outcome. It would then first be necessary to use SOSC analysis more widely, at least at one year after surgery, to correlate long term risk of ASDis to absence or presence of normal motion.

\section{Alternatives to this method of analysis}

The most common alternative has been discussed previously in this thesis, sROMs. SROMs can be used to show whether a segment is fused or still mobile, while also enabling quantification of the amount of mobility. SROMs are known to show high intra- and interindividual variability which interferes with use as a valid outcome in individual subjects [12-17]. This is clearly visible in the data on sROMs in Chapter 4, which show that sROMs are inadequate to differentiate between asymptomatic individuals and patients with symptomatic CDDD. All but one of the preoperative SROMs in the group of CDDD patients were within reported normal ranges. Also, there is no baseline available for a normal situation since the preoperative value is measured in a symptomatic patient. In large groups the variability will play a much smaller role, as the mean of the group will be taken. Therefore, one conclusion that can be drawn from the previously published RCTs is therefore that in most cases (86-100\%) motion is preserved one to two years after arthroplasty [18-22]. On the long term the number of still mobile segments decreases to around $91.5-97.1 \%$ at up to five years after surgery, $88.9 \%$ at seven years after surgery, and $81 \%$ at ten years after surgery $[1,5,23,24]$. Some of these studies did not report the amount of mobile segments (usually defined as $>2^{\circ} \mathrm{ROM}$, but report the amount of segments or with less than grade 4 heterotopic ossification. A segment with grade 3 heterotopic ossification has no bridging osteophytes, but might show $<2^{\circ} \mathrm{ROM}$, the amount of patients with a mobile segment might therefore be overestimated. 
No conclusions on quality of motion can be drawn. Quality of motion, however, might be an important aspect in assessing cervical spine mobility as well as functioning in daily life.

A second objective measure would be to measure intradiscal pressure. If one is not necessarily interested in the motion of a segment, but more in the assumed role of a fused segment on the adjacent segments (as described by Eck et al) [25]. This could be done in patients who underwent fusion and in patients who underwent arthroplasty. Measuring intradiscal pressure would elucidate a possible factor of influence on ASDis. The technology to do this is available, with wireless transmission of data so the wound can be closed in the usual way. During the procedure the surgical exposure is usually large enough to be able to do this with minimal manipulation, as the prevertebral fascia is opened already. There is however a large downside; the disc space needs to be entered. Puncturing the disc space with a 16-21G needle, or stabbing/incising the annulus (sometimes only the outer rim) is a known animal model for disc degeneration; it leads to disc degeneration within several months in rabbits, sheep and pigs [26]. False level localization by placing a $22 \mathrm{G}$ needle in the disc space and checking the level under fluoroscopy also was described as a risk factor for ASDeg in humans, resulting in a 3-fold increase for ASDeg [27]. It would therefore not be ethical to place an intradiscal sensor.

A third method to analyze motion in the spine is the use of instantaneous centers of rotation (ICR) [28-31]. An ICR is the point in space around which a vertebra rotates and translates to go from position A to position B. As motion between two vertebrae not only consists of rotation, but also translation, the ICR between position $A$ and $B$ can be different from between position $B$ and $C$, and between position $C$ and D. The series of ICRs that depicts a complete movement, e.g. the maximum flexion to maximum extension movement, forms a curve that is called the centrode of motion (Figure 1).

It has been suggested that specific abnormalities in ICR location may correspond to specific pathologies [30, 32]. In asymptomatic control subjects, this center was described to be generally fixed in the superior-inferior direction within a segment, but to translate in anterior-posterior direction during a flexion to extension movement. The ICR was reported to be located near the center of C3 for movement of C2-C3, and to move progressively superior from cranial to caudal, up to near the endplate of C7 for C6-C7 [29, 31, 33]. In recent years one of these studies did not find a change in location of the ICR in motion segments adjacent to a C5-C6 or C6-C7 arthrodesis, 7 months after surgery [34]. The shift of the ICR from cranial to caudal could be an interesting focus in the follow up of CA to investigate if the same shift that is seen in asymptomatic control subjects is present. The ICR depends on the anatomy of the motion segment, so the design of a cervical disc prosthesis (and the resulting motion constraints, such as the possibility of translation in the anterior-posterior direction) could be of influence. 


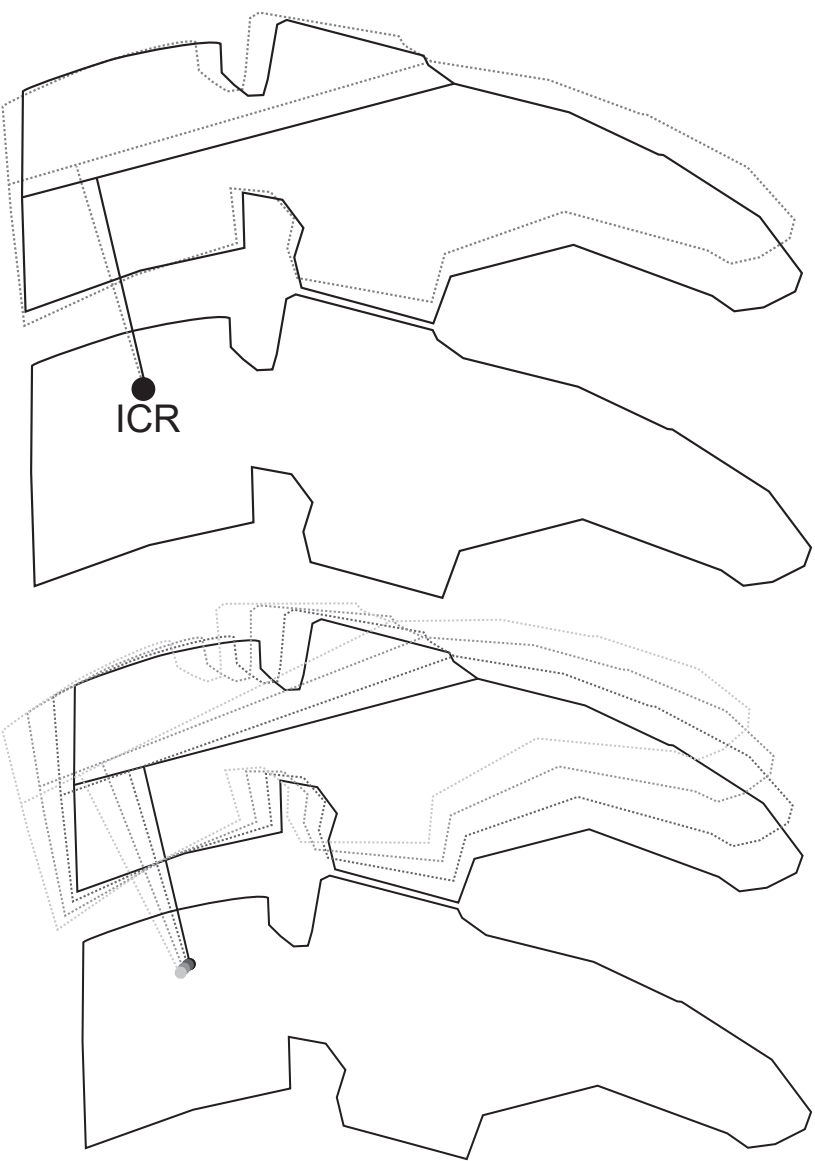

Figure 1A: example of an ICR, defined as the center of rotation around which a vertebra rotates around another vertebra.

Figure 1B: example of a centrode of motion, i.e. the motion path of the ICRs for a full motion, based on a sequence of pairs of frames that are compared.

\section{Criticism}

With regard to the primary aim of this thesis, the motion study, there are several points of critique.

The definition of normal motion has been based on a fairly small group of healthy controls ( $n=10$, recorded at two time points). However, in a subsequent study there was corroborative evidence for the defined sequence as this was also found in a second group of 10 healthy controls (also recorded at two points). Moreover, the sequence that was described in the definition of normal motion was also present in the majority of a historic control group from a descriptive study $[28,35]$. The sequence was present in about $90 \%$ 
of each of the three groups of healthy controls while it was absent in the majority of preoperative CDDD patients.

A second possible point of critique is the definition of normal motion, which is not a universal definition for normal motion in 'the cervical spine'. Only the block C4-C7 is evaluated, so no conclusions can be drawn on the rest of the cervical spine. Additionally, there might very well be other parameters that can be evaluated alongside a SOSC analysis. However, to date there is no alternative method of analysis with a specificity and sensitivity as high as SOSC analysis. Additional parameters might become available in the future, such as SOSC analysis in the upper cervical spine (block CO-C2) or in the middle cervical spine. Additionally. only rotation in the sagittal plane was evaluated. Analysis of translation in the sagittal or coronal plane, lateroflexion, and axial rotation are not evaluated with this method. A combination of parameters could increase sensitivity and/or specificity.

A third possible point of critique could be the statement that the clinical relevance of this motion pattern is debatable, as was mentioned previously in this chapter. We did not see a correlation between patient reported outcomes and presence or absence of a normal motion pattern. However, this was never the intention of the study. Similar outcomes were in fact to be expected for some outcomes (e.g. VAS scores for arm pain), as the level of neural decompression for both types of surgery is the same. The clinical relevance lies more in providing new information that can be applied to the hypothesis underlying the use of cervical arthroplasty; that it facilitates normal motion, which in turn is intended to lead to less ASDis. The first part is made more likely by this study. Although this in no way evidences that there will be an actual reduction in ASDis, it does substantiate the assumed underlying mechanism for cervical arthroplasty.

For now, the findings from this study only apply to the specific prosthesis that was used. Although other cervical disc prostheses might show similar results, this can only be proven by applying this method of analysis to patients with other types of cervical disc prostheses.

Another downside to the study is the fact that inclusion criteria and exclusion criteria were quite strict and our study population therefore does not reflect the typical CDDD patient, which diminishes generalizability of the results. This is also a strength of the study, since there are less confounders to take into account.

In retrospect, the usefulness of a control group with a high rate of fusion (which is the case for ACD as well as ACDF) is debatable. The fusion of a segment leads to a segment with no or minimal contribution to segmental motion as depicted in the graphs, which will result in a missing peak from the sequence of segmental contributions. This sequence can therefore never be normal. Simply using a group of healthy controls and a CA group would have been sufficient to be able to show presence or absence of normal motion after cervical arthroplasty. However, we would not have been able to determine whether the addition of implanting the disc prosthesis leads to a substantial increase in patients with a normal motion pattern after surgery compared to ACD. This is strengthened by the 
interesting finding that two out of the three ACD patients that did not fuse had a normal motion pattern.

With regard to the secondary aim of this thesis, comparing clinical results of arthroplasty and fusion, there are several points of critique. These points not only apply in relation to the study described in this thesis, but also in relation to the field of cervical arthroplasty in general. Since the introduction of movable cervical disc prostheses in 1991 (Bristol/Cummins cervical disc replacement, a stainless steel ball-and-socket prosthesis), several RCT's have been performed to compare the results of cervical arthroplasty with the results of the most common alternative; fusion.

Results of many of these studies, especially when pooled for meta-analysis, show statistical superiority of arthroplasty over fusion in the short term [36]. This fact is counterintuitive for patient reported outcomes as VAS scores for arm pain and the NDI, as the decompression of spinal cord and nerve root should be equal for both groups. The presumed advantage of one over the other is a long term phenomenon. The most likely explanation for the fact that there often is a statistical superiority of arthroplasty over fusion, is the presence of bias due to implementing a new technology or treatment, in combination with absence of blinding. This is a known form of ascertainment bias [37, 38]. There is the possibility of expectation bias in a patient (but it can also apply to the people who give the interventions, and assessors of the outcomes), who often expect a newer type of surgery, drug, or implant to lead to better results. The latter is referred to as novelty bias. Most of the outcomes that are used to evaluate the efficacy of this type of treatment are patient reported outcomes. That means that the patients' perceived outcome might lead to a bias to favor newer technology. The fact that for instance VAS (or NRS) for arm pain, and scores on the NDI are almost invariably lower in the arthroplasty groups is hard to explain in another way. That being said, the actual difference between the two groups is very low and cannot be considered clinically relevant, as was shown in Chapter 2 [36]. The solution to this type of bias is blinding. This is, however, difficult in surgical trials. The surgeon who is performing the treatment is obviously unblinded. A solution could be to have one surgeon perform the surgery, while a blinded physician takes care of the other aspects of treatment. This is not in agreement with the doctorpatient relation, in which a patient can (and will) expect a physician to be aware of all aspects of their diagnosis and treatment [39]. Also, a routine x-ray examination will clearly show the cervical disc prosthesis, which breaks the blinding. Additionally, it is often considered unethical to blind patients with regard to their treatment as blinding patients withholds them of the final opportunity to withdraw from the study after randomization $[40,41]$. However, the benefits of adequately blinding patients are substantial, and should at least be considered $[42,43]$. For several outcomes a blinded assessor of outcomes could be a solution, this is not the case in these studies. The majority of outcomes are patient reported outcomes and are not blinded if the patient is not blinded.

A second problem with drawing conclusions based on the outcome of the above studies, is the fact that almost all studies are industry sponsored. It has been reported that 
conflicted studies have a higher likelihood of reporting favorable results, in cervical disc prosthesis, but also in general for medical devices [44, 45]. This can also be the case for adverse events; it was also reported that studies with a conflict of interest reported lower rates of heterotopic ossification [46]. The outcome that is clinically most relevant is the amount of secondary surgery, particularly adjacent level surgery. The amount of secondary surgery can be seen as a measure of ASDis, the incidence of which one aims to lower by performing arthroplasty. The choice of performing additional surgery is quite subjective, as a treating physician is the one who decides if he offers surgical treatment in case of symptoms due to ASDis. Although very dichotomous, it cannot be seen as an objective measure.

More specific to the study described in this thesis, the small population is a definite downside. The number of subjects that was needed for the RCT was calculated based on the primary outcome. As is evident from the results of this study it was in fact sufficient to draw conclusions on the sequence of segmental contributions, this was not the case for the patient reported outcomes. The study is therefore underpowered with respect to the patient reported outcomes. In retrospect the choice could have been made to not measure patient reported outcomes at all (since the focus was on the motion study), or increase the number of included patients. However, based on the systematic review in Chapter 2 (in which data for almost 2500 patients were included), as well as the individual RCTs on which it is based, it is unlikely that (even substantially) increasing the group sizes would have led to a significant and clinically relevant difference in our RCT.

\section{Future plans}

There are several possible ways to continue this research. First, it would be very interesting to repeat the SOSC analysis on the long term in our population. The population from the RCT that we described is at 2 to 10 years after surgery at the moment. By using a long term SOSC analysis in the same population it would be possible to see whether there is a change in the sequence over time. It would also be possible to see if there is a correlation between the presence/absence of a normal sequence and results of patient reported outcomes in the long term, or the amount of secondary surgery. The previously discussed limited sampled size is a severe limiting factor, only a very clear correlation can be identified this way.

Second, now that a normal sequence has been described, the method could be used in other populations with varying cervical spine pathologies. For instance in patients with myelopathy due to cervical canal stenosis, or to assess stability after trauma.

Third, it would be interesting to investigate if a similarly consistent normal sequence can be identified for the lumbar spine, which could then serve as a basis for further study in the lumbar spine in varying pathologies. 
In the fourth place, it would be interesting to further investigate the origin of the sequence that was found. As already mentioned in the previous paragraph, it intuitively is the way to move the cervical spine with the least effort, so with maximum 'help' of gravity. This would mean that in a different position, for instance in a supine position, the sequence would be very different due to the different vector of gravity.

This method could be used on different populations of CA patients, with varying types of cervical disc prostheses. This way it would be possible to assess if there is a difference in the amount of patients who return to a normal sequence of segmental contributions. Ideally this would be combined with short and longer term follow up in these different populations to not only increase power, but to also increase external validity of this method in CA patients.

Finally, since it is not uncommon for CDDD patients to have multilevel pathology, it would also be interesting to investigate the motion pattern in multilevel cervical arthroplasty. 


\section{References}

1. Hisey, M.S., et al., Prospective, Randomized Comparison of One-level Mobi-C Cervical Total Disc Replacement vs. Anterior Cervical Discectomy and Fusion: Results at 5-year Follow-up. Int J Spine Surg, 2016. 10: p. 10.

2. Janssen, M.E., et al., ProDisc-C Total Disc Replacement Versus Anterior Cervical Discectomy and Fusion for Single-Level Symptomatic Cervical Disc Disease: Seven-Year Follow-up of the Prospective Randomized U.S. Food and Drug Administration Investigational Device Exemption Study. J Bone Joint Surg Am, 2015. 97(21): p. $1738-47$.

3. Sasso, W.R., et al., Long-term Clinical Outcomes of Cervical Disc Arthroplasty: A Prospective, Randomized, Controlled Trial. Spine (Phila Pa 1976), 2017. 42(4): p. 209-216.

4. Phillips, F.M., et al., Long-term Outcomes of the US FDA IDE Prospective, Randomized Controlled Clinical Trial Comparing PCM Cervical Disc Arthroplasty With Anterior Cervical Discectomy and Fusion. Spine (Phila Pa 1976), 2015. 40(10): p. 674-83.

5. Coric, D., et al., Prospective, randomized multicenter study of cervical arthroplasty versus anterior cervical discectomy and fusion: 5-year results with a metal-on-metal artificial disc. J Neurosurg Spine, 2018. 28(3): p. 252-261.

6. Thomas Graven-Nielsen, P.S.L.A.-N., P. Svensson, and L. Arendt-Nielsen, Effect of Muscle Pain on Motor Control: A Human Experimental Approach. Advances in Physiotherapy, 2000. 2(1): p. 26-38.

7. Lund, J.P., et al., The pain-adaptation model: a discussion of the relationship between chronic musculoskeletal pain and motor activity. Can J Physiol Pharmacol, 1991. 69(5): p. 683-94.

8. Tsang, S.M., G.P. Szeto, and R.Y. Lee, Altered spinal kinematics and muscle recruitment pattern of the cervical and thoracic spine in people with chronic neck pain during functional task. J Electromyogr Kinesiol, 2014. 24(1): p. 104-13.

9. Hodges, P.W. and C.A. Richardson, Inefficient muscular stabilization of the lumbar spine associated with low back pain. A motor control evaluation of transversus abdominis. Spine (Phila Pa 1976), 1996. 21(22): p. 2640-50.

10. MacDonald, D., G.L. Moseley, and P.W. Hodges, Why do some patients keep hurting their back? Evidence of ongoing back muscle dysfunction during remission from recurrent back pain. Pain, 2009. 142(3): p. 1838.

11. Kuwahara, W., et al., Characteristics of thoracic and lumbar movements during gait in lumbar spinal stenosis patients before and after decompression surgery. Clin Biomech (Bristol, Avon), 2016. 40: p. 45-51.

12. Van Mameren, $H_{\text {., }}$ et al., Cervical spine motion in the sagittal plane (I) range of motion of actually performed movements, an X-ray cinematographic study. Eur J Morphol, 1990. 28(1): p. 47-68.

13. Lind, B., et al., Normal range of motion of the cervical spine. Arch Phys Med Rehabil, 1989. 70(9): p. 692-5.

14. Dvorak, J., et al., In vivo flexion/extension of the normal cervical spine. J Orthop Res, 1991. 9(6): p. 828-34.

15. Dvorak, J., et al., Functional radiographic diagnosis of the cervical spine: flexion/extension. Spine (Phila Pa 1976), 1988. 13(7): p. 748-55.

16. Bhalla, S.K. and E.H. Simmons, Normal ranges of intervertebral-joint motion of the cervical spine. Can J Surg, 1969. 12(2): p. 181-7.

17. Aho, A., O. Vartiainen, and O. Salo, Segmentary antero-posterior mobility of the cervical spine. Ann Med Intern Fenn, 1955. 44(4): p. 287-99.

18. Tu, T.H., et al., Heterotopic ossification after cervical total disc replacement: determination by CT and effects on clinical outcomes. J Neurosurg Spine, 2011. 14(4): p. 457-65.

19. Goffin, J., et al., Intermediate follow-up after treatment of degenerative disc disease with the Bryan Cervical Disc Prosthesis: single-level and bi-level. Spine (Phila Pa 1976), 2003. 28(24): p. 2673-8.

20. Porchet, F. and N.H. Metcalf, Clinical outcomes with the Prestige II cervical disc: preliminary results from a prospective randomized clinical trial. Neurosurg Focus, 2004. 17(3): p. E6.

21. Mummaneni, P.V., et al., Clinical and radiographic analysis of cervical disc arthroplasty compared with allograft fusion: a randomized controlled clinical trial. J Neurosurg Spine, 2007. 6(3): p. 198-209. 
22. Anderson, P.A., et al., The Bryan Cervical Disc: wear properties and early clinical results. Spine J, 2004. 4(6 Suppl): p. 303S-309S.

23. Radcliff, K., et al., Long-term Evaluation of Cervical Disc Arthroplasty with the Mobi-C(c) Cervical Disc: A Randomized, Prospective, Multicenter Clinical Trial with Seven-Year Follow-up. Int J Spine Surg, 2017. 11: p. 31.

24. Dejaegher, J., et al., 10-year follow-up after implantation of the Bryan Cervical Disc Prosthesis. Eur Spine J, 2017. 26(4): p. 1191-1198.

25. Eck, J.C., et al., Biomechanical study on the effect of cervical spine fusion on adjacent-level intradiscal pressure and segmental motion. Spine (Phila Pa 1976), 2002. 27(22): p. 2431-4.

26. Singh, K., K. Masuda, and H.S. An, Animal models for human disc degeneration. Spine J, 2005. 5(6 Suppl): p. 267S-279S.

27. Nassr, A., et al., Does incorrect level needle localization during anterior cervical discectomy and fusion lead to accelerated disc degeneration? Spine (Phila Pa 1976), 2009. 34(2): p. 189-92.

28. Bogduk, N. and S. Mercer, Biomechanics of the cervical spine. I: Normal kinematics. Clin Biomech (Bristol, Avon), 2000. 15(9): p. 633-48.

29. Amevo, B., D. Worth, and N. Bogduk, Instantaneous axes of rotation of the typical cervical motion segments: a study in normal volunteers. Clin Biomech (Bristol, Avon), 1991. 6(2): p. 111-7.

30. Bogduk, N., B. Amevo, and M. Pearcy, A biological basis for instantaneous centres of rotation of the vertebral column. Proc Inst Mech Eng H, 1995. 209(3): p. 177-83.

31. Anderst, W.J., et al., Cervical spine intervertebral kinematics with respect to the head are different during flexion and extension motions. J Biomech, 2013. 46(8): p. 1471-5.

32. Amevo, B., C. Aprill, and N. Bogduk, Abnormal instantaneous axes of rotation in patients with neck pain. Spine (Phila Pa 1976), 1992. 17(7): p. 748-56.

33. van Mameren, $H_{\text {., }}$ et al., Cervical spine motion in the sagittal plane. II. Position of segmental averaged instantaneous centers of rotation--a cineradiographic study. Spine (Phila Pa 1976), 1992. 17(5): p. 467-74.

34. Anderst, W., et al., Motion path of the instant center of rotation in the cervical spine during in vivo dynamic flexion-extension: implications for artificial disc design and evaluation of motion quality after arthrodesis. Spine (Phila Pa 1976), 2013. 38(10): p. E594-601.

35. Van Mameren, H., Motion patterns in the cervical spine. 1988, Maastricht University.

36. Boselie, T.F., et al., Arthroplasty versus fusion in single-level cervical degenerative disc disease: a Cochrane review. Spine (Phila Pa 1976), 2013. 38(17): p. E1096-107.

37. Schulz, K.F., et al., Empirical evidence of bias. Dimensions of methodological quality associated with estimates of treatment effects in controlled trials. JAMA, 1995. 273(5): p. 408-12.

38. Colditz, G.A., J.N. Miller, and F. Mosteller, How study design affects outcomes in comparisons of therapy. I: Medical. Stat Med, 1989. 8(4): p. 441-54.

39. Goold Susan, D. and M. Lipkin, The Doctor-Patient Relationship. Journal of General Internal Medicine, 1999. 14(S1): p. 26-33.

40. Nardini, C., The ethics of clinical trials. Ecancermedicalscience, 2014. 8: p. 387.

41. McLeod, R.S., Issues in surgical randomized controlled trials. World J Surg, 1999. 23(12): p. $1210-4$.

42. de Kunder, S.L., et al., A protocol of a randomized controlled multicenter trial for surgical treatment of lumbar spondylolisthesis: the Lumbar Interbody Fusion Trial (LIFT). BMC Musculoskelet Disord, 2016. 17(1): p. 417.

43. Arts, M.P., et al., The NEtherlands Cervical Kinematics (NECK) trial. Cost-effectiveness of anterior cervical discectomy with or without interbody fusion and arthroplasty in the treatment of cervical disc herniation; a double-blind randomised multicenter study. BMC Musculoskelet Disord, 2010. 11: p. 122.

44. Narain, A.S., et al., Cervical disc arthroplasty: do conflicts of interest influence the outcome of clinical studies? Spine J, 2017. 17(7): p. 1026-1032.

45. Bhandari, M., et al., Hierarchy of evidence: differences in results between non-randomized studies and randomized trials in patients with femoral neck fractures. Arch Orthop Trauma Surg, 2004. 124(1): p. 10-6.

46. Alvin, M.D., et al., Cervical arthroplasty: a critical review of the literature. Spine J, 2014. 14(9): p. $2231-45$. 

Valorisation 

The mean annual incidence of cervical radiculopathy is reported to be about 83 per 100,000 people, with a peak incidence of 202 per 100,000 for people aged 50 to 54 years. The prevalence is about 3.5 per 1,000 people, also with a peak in the sixth decade $[1,2]$.

The natural course of a cervical radiculopathy is benign, with a spontaneous resolution of complaints in $60 \%$ of the patients after 6 weeks of conservative treatment, and in $83 \%$ of the patients after 2 years of conservative treatment [3]. A recurrence of a cervical radiculopathy has been described in $31.9 \%$ of the conservatively treated patients. Many of these were isolated recurrences [1]. The reported proportion of patients who are eventually treated surgically ranges from $8 \%$ to $35 \%[1,4,5]$. In the Netherlands, around 2,000 patients with cervical radiculopathy are operated upon each year. Based on these numbers, the cost of surgical treatment of cervical radiculopathy was estimated to lie around 30 million euros [6]. Of the surgically treated patients, around $90 \%$ was satisfied with the result at 6 to 8 weeks after surgery [7-9].

Although many of the patients recover from the complaints with conservative therapy alone or with surgical treatment, the burden of disease is high in these patients whilst the complaints are still present. The actual burden of cervical degenerative disc disease for society is not known. The costs-of-illness for neck pain in the Netherlands have been reported to be $1 \%$ of the total health care expenditures in 1996 [10], the largest part of these costs were related to disability and absenteeism (35\% and 65\%, respectively). The percentage of these patients with neck pain that had a cervical radiculopathy is not reported. The largest company in the Netherlands that supports employers and employees in illness-related absenteeism, ArboNed, reported a total of 1079 people absent from work due to a cervical radiculopathy in the period 2015 to October 2018. This was about $1.2 \%$ of the total amount of people that were reported to be absent from work in that period. On average, they were absent from work for 246 days (personal communication with ArboNed). One day of absence from work is usually calculated to cost around 250 euros, which leads to average costs of absenteeism of 61.500,- euros per patient. Given that the market share of ArboNed lies around 23\%, the actual number of patients that were absent from work during that period due to a cervical radiculopathy can be extrapolated to be four times higher, with annual costs solely due to absenteeism of 66 million euros.

An RCT investigating cost-effectiveness in ACDF and dorsal foraminotomy in cervical radiculopathy is currently including patients, and could shed more light on the actual societal cost of cervical radiculopathy [11]. This will of course only be applicable to a subset of patients, the ones who end up being surgically treated. Another RCT investigating costeffectiveness of three types of surgery for cervical radiculopathy, 1) ACD with interbody fusion, 2) ACD without interbody fusion and 3) arthroplasty has finished including patients, but results have not been reported yet [12]. 


\section{Secondary surgery}

The incidence ASDis in patients who have undergone fusion surgery is reported to be $2.9 \%$ per year, and $25 \%$ in ten years [13]. Prevention of new radicular complaints in surgically treated patients could prevent new episodes of discomfort, disability, absenteeism, as well as the accompanying costs. If there are differences in cost-effectiveness between the different surgical treatments of cervical radiculopathy the studies mentioned in the previous paragraph can hopefully shed more light on it [11, 12]. Given the fact that the intended goal of cervical arthroplasty is preventing secondary complaints due to adjacent segment disease (ASDis) the societal burden in the long term could be lower in the arthroplasty group.

The idea behind cervical arthroplasty is to reduce adjacent level surgery by preserving motion in the operated segment. This thesis, however showed that the imaging technique is very good, but interpretation of normal motion remains difficult. An actual reduction in ASDis can only be proven with long-term randomized studies of good quality. Long-term data are slowly becoming available and several of them report lower rates of secondary surgery in the arthroplasty group, when compared to the fusion group [14]. We are currently performing a systematic review on the long term results of RCT's comparing arthroplasty to fusion [15]. This will hopefully shed more light on this subject. However, irrespective of a difference in secondary surgery that might be present, it is yet unknown whether this can be linked to the postulated preservation of motion.

\section{Timing of evaluation}

A reduction of secondary surgery is based on the assumption that arthroplasty preserves physiological motion, and that fusion plays a causal role in the development of ASDis. A definition of a physiological motion pattern was reported in this thesis. This motion pattern was present in most asymptomatic individuals, while absent in the majority of CDDD patients. It was present in the majority of these patients after surgical decompression with placement of a cervical disc prosthesis.

Although this is only one parameter of physiological motion there currently is no alternative that can differentiate more reliably between CDDD patients and asymptomatic individuals. It could therefore be used as a tool to evaluate patients after surgery. While clinical results take 5 to 10 years of follow-up (and even then a systematic review appears to be necessary to be able to reach statistical significance), the motion pattern of a patient can be determined one year after surgery. However, to be able to draw conclusions based on the sequence of segmental contributions data from one year after surgery, a good correlation would have to be shown between the motion pattern and long term outcome.

To determine whether this method is useful as an early parameter to determine if a type of prosthesis (or other variables such a location of the disc prosthesis within the disc 
space, or concomitant degenerative changes) facilitates a physiological motion pattern, a much larger sample size would be necessary given the low annual incidence of ASDis and the large amount of variables that might influence results. Ideally this method of analysis would be used more widely, in future (or ongoing) RCT's.

It has already been shown that cervical arthroplasty preserves motion in the majority of patients, primarily by use of segmental range of motions (sROMs). Given the known high variability of sROMs the data are not useful to determine if physiological motion is present or absent. Without information whether the motion is physiological or not, it cannot be concluded that motion preservation on itself might be enough to prevent secondary surgery. It is possible that patients with a physiological motion pattern have a lower risk of secondary surgery then patient with preserved motion but without a physiological motion pattern.

\section{Expertise in analysis of sequence of segmental contributions}

There are several companies (e.g. Medical Metrics Inc., ICON) that offer analysis of various types of imaging such as digital subtraction angiography (DSA), X-ray CT, single photon emission computed tomography (SPECT), positron emission tomography (PET), optical techniques and magnetic resonance imaging (MRI). These Imaging Core Labs (ICLs) support trials by collecting, analysing (blinded) and archiving the imaging data for a complete trial. Some of these ICLs are commercial, others are academic / not-for-profit. In the fusion and arthroplasty studies these companies primarily analyse flexion- and extension radiographs (to determine sROMs and translations in the sagittal plane). As was stated above, these outcomes are less than optimal for use in individual patients. None of these ICLs currently offer analysis of sequence of segmental contributions.

A similar type of Imaging Lab could be set up to offer analysis of sequence of segmental contributions. Facilitating use of this method of analysis in other studies could help to make it more widely used.

\section{Impact for future health care if a relation between preservation of physiological motion and secondary surgery would be proven}

If a physiological motion pattern would be proven to correlate with a decrease in the risk of secondary surgery there could be a role for analysis of sequence of segmental contributions in earlier stages of clinical trials of new types of cervical disc prostheses. Because the sequence of segmental contributions can be determined one year after surgery, while results on secondary surgery take several years (and large groups) to become known, valuable time and resources could be saved. If a new type of prosthesis preserves motion, but without a physiological motion pattern, design changes could be implemented. 
Ideally, one would want to be able to do this even before clinical trials, in the design phase. It would then be necessary to find out which parameters determine the physiological motion pattern (e.g. the location of the centre of rotation of a prosthesis, whether or not it needs to be able to accommodate translation in the coronal/sagittal/transverse plane, but also patient specific variables such as the size and shape of the intervertebral space, orientation and shape of the facet joints, and alignment of the spine). A very large amount of source data would be necessary to determine this, which is not realistic at this time. A second option would be a combination with Finite Element Analysis to create a model of the cervical spine in which the influence of the parameters that were mentioned in the previous paragraph on the sequence of segmental contributions can be determined, and to validate this model using already available data. However, a highly complex model would be required to be able to do this. 


\section{References}

1. Radhakrishnan, K., et al., Epidemiology of cervical radiculopathy. A population-based study from Rochester, Minnesota, 1976 through 1990. Brain, 1994. 117 ( Pt 2): p. 325-35.

2. Salemi, G., et al., Prevalence of cervical spondylotic radiculopathy: a door-to-door survey in a Sicilian municipality. Acta Neurol Scand, 1996. 93(2-3): p. 184-8.

3. Kuijper, B., et al., Degenerative cervical radiculopathy: diagnosis and conservative treatment. A review. Eur J Neurol, 2009. 16(1): p. 15-20.

4. Saal, J.S., J.A. Saal, and E.F. Yurth, Nonoperative management of herniated cervical intervertebral disc with radiculopathy. Spine (Phila Pa 1976), 1996. 21(16): p. 1877-83.

5. Wolff, M.W. and L.A. Levine, Cervical radiculopathies: conservative approaches to management. Phys Med Rehabil Clin N Am, 2002. 13(3): p. 589-608, vii.

6. Vleggeert-Lankamp, C.L.A. and B. Kuijper, Cervical radicular syndrome: a general reflection on contemporary treatment. Tijdschrift voor Neurologie en Neurochirurgie, 2013(114): p. 36-42.

7. Dowd, G.C. and F.P. Wirth, Anterior cervical discectomy: is fusion necessary? J Neurosurg, 1999. 90(1 Suppl): p. 8-12

8. Wirth, F.P., et al., Cervical discectomy. A prospective analysis of three operative techniques. Surg Neurol, 2000. 53(4): p. 340-6; discussion 346-8.

9. Nandoe Tewarie, R.D., R.H. Bartels, and W.C. Peul, Long-term outcome after anterior cervical discectomy without fusion. Eur Spine J, 2007. 16(9): p. 1411-6.

10. Borghouts, J.A., et al., Cost-of-illness of neck pain in The Netherlands in 1996. Pain, 1999. 80(3): p. 629-36.

11. Broekema, A.E., et al., Study protocol for a randomised controlled multicentre study: the Foraminotomy ACDF Cost-Effectiveness Trial (FACET) in patients with cervical radiculopathy. BMJ Open, 2017. 7(1): p. e012829.

12. Arts, M.P., et al., The NEtherlands Cervical Kinematics (NECK) trial. Cost-effectiveness of anterior cervical discectomy with or without interbody fusion and arthroplasty in the treatment of cervical disc herniation; a double-blind randomised multicenter study. BMC Musculoskelet Disord, 2010. 11: p. 122.

13. Hilibrand, A.S., et al., Radiculopathy and myelopathy at segments adjacent to the site of a previous anterior cervical arthrodesis. J Bone Joint Surg Am, 1999. 81(4): p. 519-28.

14. Boselie, T.F. and H. van Santbrink, Arthroplasty in cervical degenerative disc disease: fulfilling its long-term promise? J Spine Surg, 2016. 2(4): p. 359-361.

15. Boselie TFM, et al., Arthroplasty versus fusion for single-level cervical degenerative disc disease (protocol for update) Cochrane Database of Systematic Reviews 2016(Issue 9): p. CD012339. . 

Summary 

Cervical degenerative disc disease (CDDD) is degeneration of a cervical intervertebral disc and/or the adjoining vertebral bodies. Possible sequelae are a bulging or herniated intervertebral disc, foraminal narrowing due to loss of disc space height or osteophyte formation, or a combination of these. A common resulting clinical symptom is cervical radiculopathy. The burden of disease due to cervical radiculopathy is high, for patients as well as society. Surgical treatment is an option in case of inadequate relief of symptoms after a period of non-operative treatment. For decades the most commonly performed surgery for this indication was an anterior cervical discectomy without/with promoting fusion (ACD/ACDF). Both of these options have a high rate of fusion of the motion segment.

Cervical disc prostheses have been developed to preserve of motion in the operated segment after ACD/ACDF. The intended goal is a reduction in adjacent segment disease (ASDis), which has been reported to occur in $25 \%$ of the patients in the ten years after surgery. It has been suggested that degeneration in the adjacent segment is accelerated as a result of the fusion in the operated segment. This has not been proven in vivo, and is largely based on biomechanical studies in cadavers.

The protective effect of cervical disc prosthesis is based on medical device manufactures' claims that these prostheses facilitate normal motion in the operated segment. However, these claims have not been proven. Therefore, we aimed to investigate motion after placement of a cervical disc prosthesis ('arthroplasty'). A couple of methods to evaluate motion in the cervical spine were considered. The first was measurement of segmental range of motion (sROM), which is the most common method in trials investigating cervical spine mobility. The second was determining the sequence of segmental contributions to flexion and/or extension. The first is known to show large intra- and interindividual variability, which severely limits its use in individual patients. The second has previously been reported to show a much more consistent pattern, with little intra- and interindividual variability.

The aim of the thesis was therefore to investigate if arthroplasty restores a normal sequence of segmental contributions after anterior cervical discectomy (chapter 1).

\section{Clinical results of arthroplasty versus fusion}

Arthroplasty has been the focus of several clinical trials, and is usually compared to ACDF. Long term studies were not available when the subject focus of this research trajectory was determined. A possible reduction in ASDis in the long term can only be awaited if short term results are equal to, or better than ACDF, the most commonly performed alternative. 
This was why a systematic review was performed. Nine randomized controlled trials (RCTs) were included in this systematic review, all directly comparing arthroplasty to fusion. These nine studies included a total of 2.400 patients. Duration of follow up was a maximum of one to two years.

Although several outcomes were statistically in favour of arthroplasty, no clinical relevant differences were seen except a significantly higher amount of patients with preserved motion. The authors therefore concluded that cervical disc prostheses should only be used in trials until long term data would be available (chapter 2).

\section{Motion analysis}

Analysis of the sequence of segmental contributions has previously been performed by manual imputation of vertebral landmarks on all frames of $x$-ray cinematographic recordings of a flexion or extension movement, using a digitizer tablet. This is extremely labour intensive. In conjunction with Technical University Eindhoven (TU/e) an image analysis assisted method was developed to track the skull and cervical vertebrae throughout $\mathrm{x}$ ray cinematographic recordings of a flexion or extension movement. This method uses a normalized gradient field to compare the orientation of a used defined template area between frames of such a recording, which speeds up the process markedly (chapter 3 ).

This method was then applied to x-ray cinematographic recordings of full 'bending' flexion movements (FCRs) and extension movement (ECRs) in healthy volunteers. These data were used to determine a definition of a 'normal' sequence of segmental contributions in the lower cervical spine (C4-C7). A specific sequence in ECRs was found to be most consistent. This was the sequence of a maximum contribution of C4-C5, followed by C5C6 and finally C6-C7 during the last phase of the extension movement (chapter 4).

This definition was then used to score the sequence of segmental contributions in twenty healthy controls, and ten preoperative patients with CDDD in a blinded fashion to be able to determine reproducibility, sensitivity and specificity in determining between healthy controls and preoperative CDDD patients. This was done by five blinded independent observers at three time points. Reproducibility was high with a Fleiss kappa of 0.80-0.84 (range of $95 \% \mathrm{Cl}: 0.66-0.98$ ), sensitivity and specificity were also high, with an average sensitivity of $90 \%$ (range of $95 \% \mathrm{Cl}: 78.4-99.8 \%$ ), and an average specificity of $85 \%$ (range of $95 \% \mathrm{Cl}: 72.9-96.4 \%$ ) (chapter 4).

\section{Randomized controlled trial}

A protocol was written for a RCT comparing arthroplasty to the most commonly performed alternative at that time at our centre, ACD. Primary outcome was the sequence of segmental contributions. Additionally, several patient reported outcomes, as well as 
neurologic examinations were determined as secondary outcomes. A total of twelve patients was calculated to be necessary for each of the two groups, taking an attrition rate of $20 \%$ into account (chapter 5 ).

The first patient was operated in December 2007, the last follow up of the last patient took place in October 2015. At one year after surgery, the sequence of segmental contributions in C4-C7 that is described in asymptomatic subjects was present in 8/10 (80\%) of the CA group, and in 2/10 (20\%) of the ACD group. Fusion rate was $0 / 10(0 \%)$ in the CA group, and $7 / 10(70 \%)$ in the ACD group. There were no differences in patient reported outcomes between the two treatments, nor in any of the subgroup analyses (chapter 6).

\section{Conclusion}

The aim of the thesis was to investigate if arthroplasty restores a normal sequence of segmental contributions in CDDD patients. At one year after surgery, the majority $(80 \%)$ of the patients with a prosthesis was shown to have a motion pattern as was defined based on healthy controls. However, the clinical relevance of restoring/preserving a normal motion pattern in the short term is doubtful. If fusion at the operated segment indeed plays a role in the development of ASDis, preservation of motion with a normal motion pattern may be a prerequisite for prevention of ASDis in the long term. It would be beneficial if the analysis of the sequence of segmental contributions would be used more commonly, as it is the most consistent parameter for evaluating the presence of physiologic motion in the caudal cervical spine. 

Samenvatting 

Symptomatische cervicale discus degeneratie (CDDD) omhelst degeneratieve afwijkingen van een cervicale discus en/of de twee aangrenzende wervels. Dit kan resulteren in uitpuilende of herniërende discus, versmalling van het neuroforamen door hoogteverlies van de discus, vorming van osteofyten, of een combinatie hiervan. Dit kan leiden tot een radiculopathie. De ziektelast van een cervicale radiculopathie is hoog, zowel voor de patient als voor de maatschappij. Chirurgische behandeling is een optie indien er onvoldoende verbetering van de klachten optreedt onder een niet-chirurgisch beleid. Decennialang was de meest voorkomende behandeling een anterieure cervicale discectomie, met of zonder fusie bevorderende maatregelen (ACD/ACDF). Zowel de ACD als de ACDF leiden in veel gevallen tot fusie van het bewegingssegment.

Cervicale discusprothesen zijn ontwikkeld om de mobiliteit van het bewegingssegment te behouden na een ACD. Het doel hiervan is het verminderen van de hoeveelheid patiënten die op een later moment klachten ontwikkeld door degeneratie van een aangrenzend bewegingssegment (ASDis), waarvan beschreven is dat dit in de tien jaar na de ingreep bij $25 \%$ van de patiënten voorkomt. Er is gesuggereerd dat degeneratie van een aangrenzend bewegingssegment versneld wordt door fusie in het geopereerde segment. Dit is nooit in vivo aangetoond, en is grotendeels gebaseerd op biomechanisch onderzoek in kadavers.

Een beschermend effect van cervicale discusprothesen is gebaseerd op de bewering van fabrikanten dat deze leiden tot normale mobiliteit van een bewegingssegment na de ingreep. Dit is echter nooit bewezen. Wij wilden derhalve de mobiliteit na het implanteren van een discusprothese ("arthroplasty") onderzoeken. Daarvoor werden twee methoden voor het onderzoeken van de beweeglijkheid van de cervicale wervelkolom overwogen. De eerste was het meten van de segmentale beweeglijkheid (sROM), wat de meest gebruikte methode is bij studies die beweeglijkheid van de cervicale wervelkolom onderzoeken. De tweede was het bepalen van de volgorde waarin de afzonderlijke bewegingssegmenten bijdragen aan flexie/extensie van de cervicale wervelkolom. Van de eerste methode is bekend dat deze een zeer hoge intra- en interindividuele variabiliteit heeft, waardoor het nut in individuele patiënten zeer beperkt is. Van de tweede methode is eerder beschreven dat deze een veel consistenter patroon laat zien, met een beperkte intra- en interindividuele variabiliteit.

Het doel van dit proefschrift was derhalve om te onderzoeken of door arthroplasty de normale volgorde van bewegen in de cervicale wervelkolom hersteld wordt (hoofdstuk 1).

\section{Klinische resultaten van arthroplasty versus fusie}

$\mathrm{Er}$ is in meerdere studies gekeken naar arthroplasty, waarbij er doorgaans vergeleken wordt met ACDF ('fusie'). Er waren nog geen studies met lange termijn resultaten beschikbaar toen het onderwerp van dit proefschrift bepaald werd. Er kan alleen afgewacht worden of er een afname van het aantal patiënten met ASDis na het plaatsen van een 
discusprothese is, indien arthroplasty vergelijkbare, of betere, resultaten laat zien dan fusie op de korte termijn.

Er werd derhalve besloten tot het verrichten van een systematische review. Hierin werden negen gerandomiseerde studies (RCT's) geïncludeerd, die allen arthroplasty rechtstreeks vergeleken met fusie. In deze negen studies werden in totaal 2.400 patiënten geincludeerd. De patiënten werden maximaal één of twee jaar na de operatie opgevolgd.

Hoewel meerdere resultaten significant beter waren voor de arthroplasty groep werden er geen klinisch relevante verschillen gezien behalve een veel groter aantal patiënten met intacte beweeglijkheid van het geopereerde segment. De auteurs concludeerden daarom dat cervicale discusprothesen alleen in studieverband geïmplanteerd zouden moeten worden tot er voldoende lange termijn resultaten beschikbaar zouden zijn (hoofdstuk 2).

\section{Bewegingsanalyse}

In het verleden werd de volgorde van segmentale bijdragen geanalyseerd door middel van het handmatig aangeven van karakteristieke punten van wervels op alle beelden van een röntgenopname van een flexie of extensie beweging. Dit gebeurde op een digitizer en is zeer arbeidsintensief. Samen met de Technische Universiteit Eindhoven (TU/e) werd een beeldherkenningsalgoritme geassisteerde methode ontwikkeld waarmee de schedel en alle cervicale wervels gevolgd kunnen worden op alle beelden van een röntgenopname van een flexie of extensiebeweging. Deze methode makt gebruik van een genormaliseerd gradiëntveld in een door de gebruiker aangegeven gebied, en vergelijkt dit met de andere beelden van een röntgenopname. Het proces wordt hierdoor grotendeels geautomatiseerd en daardoor sneller en minder arbeidsintensief (hoofdstuk 3).

Deze methode werd vervolgens toegepast op röntgenopnamen van flexie bewegingen (FCR's) en röntgenopnamen van extensie bewegingen (ECR's) bij klachtenvrije vrijwilligers. De resultaten van deze analyse werden gebruikt om een definitie van een 'normale' volgorde van segmentale bijdragen te bepalen in de caudale cervicale wervelkolom (C4-C7). Een bepaalde volgorde in de ECR's bleek het meest consistent. Dit was een maximale bijdrage van C4-C5, gevolgd door C5-C6, en dan C6-C7, tijdens de laatste fase van de extensie beweging (hoofdstuk 4).

Deze definitie werd vervolgens gebruikt om geblindeerd de volgorde van segmentale bijdragen te scoren in opnamen van 20 klachtenvrije vrijwilligers en tien preoperatieve patiënten met CDDD. Hierdoor werden reproduceerbaarheid, sensitiviteit en specificiteit bepaald bij het maken van onderscheid tussen klachtenvrije mensen en mensen met CDDD. Het scoren gebeurde geblindeerd door vijf onafhankelijke observatoren, op drie tijdpunten. De reproduceerbaarheid was hoog met een Fleiss kappa van 0.80-0.84 (gren- 
zen van het 95\% Cl: 0.66-0.98), sensitiviteit en specificiteit waren ook hoog, met een gemiddelde sensitiviteit van 90\% (95\% Cl: 78.4-99.8\%), en een gemiddelde specificiteit van $85 \%$ (95\% Cl: 72.9-96.4\%) (hoofdstuk 4).

\section{Randomized controlled trial}

Er werd een protocol geschreven voor een RCT waarin arthroplasty met de meest gebruikte behandeling in ons centrum op dat moment, ACD, vergeleken zou worden. Primaire uitkomstmaat was de volgorde van segmentale bijdragen. Als secundaire uitkomstmaten zouden meerdere enquêtes ingevuld worden door de patiënten, en zou er een neurologisch onderzoek op enkele momenten plaatsvinden. Er werd berekend dat er twaalf patiënten nodig waren per groep, rekening houdend met een loss to follow up van 20\% (hoofdstuk 5).

De eerste patiënt werd geopereerd in december 2007, de laatste controle bij de laatste patiënt vond plaats in oktober 2015. Eén jaar na de ingreep was de volgorde van segmentale bijdragen in C4-C7 die beschreven is in klachtenvrije personen aanwezig in 8/10 (80\% van de patiënten in de arthroplasty groep en in 2/10 (20\%) van de patiënten in de ACD groep. Er was fusie opgetreden bij 0/10 (0\%) van de patiënten in de arthroplasty groep, en in 7/10 (70\%) van patiënten in de ACD groep. Er werd geen klinisch verschil in uitkomst gevonden tussen de twee behandelingen in de studie, noch in de subgroep analyses (hoofdstuk 6).

\section{Conclusie}

Het doel van dit proefschrift was om te onderzoeken of bij CDDD patiënten de volgorde van segmentale bijdragen hersteld wordt door implantatie van een discusprothese. Eén jaar na de ingreep laat de meerderheid van de patiënten in de arthroplasty groep (80\%) een normale volgorde van segmentale bijdragen in de caudale wervelkolom zien. Op de korte termijn is de klinische relevantie van de aanwezigheid van een normale volgorde twijfelachtig. Als fusie van een geopereerd segment inderdaad een rol speelt bij het ontstaan van ASDis zou het behouden van beweeglijkheid in het segment met herstellen van een normale volgorde van segmentale bijdragen een vereiste zijn om ASDis op de lange termijn te voorkomen. Het zou goed zijn als de analyse van de volgorde van segmentale bijdragen vaker gebruikt zou worden, aangezien het de meest consistente parameter is voor het vaststellen van normale beweeglijkheid in de caudale cervicale wervelkolom. 



\section{List of abbreviations}

\begin{tabular}{|c|c|}
\hline ACD & anterior cervical discectomy \\
\hline ACDF: & anterior cervical discectomy with fusion \\
\hline ACDA & anterior cervical discectomy with arthroplasty \\
\hline ASD & adjacent segment degeneration \\
\hline ADD & adjacent disc disease \\
\hline ASDeg & adjacent segment degeneration \\
\hline ASDis & adjacent segment disease (= symptomatic adjacent segment degeneration) \\
\hline CA & cervical arthroplasty \\
\hline CBRG & Cochrane Back Review Group \\
\hline CDDD & cervical degenerative disc disease \\
\hline CENTRAL & Cochrane Central Register of Controlled Trials \\
\hline $\mathrm{Cl}$ & confidence interval \\
\hline FDA & US Food and drug administration \\
\hline EBMR & Evidence-Based Medicine Reviews \\
\hline ECR & extension cinematographic recording \\
\hline EMBASE & Excerpta Medica dataBASE \\
\hline FCR & flexion cinematographic recording \\
\hline FFER & fluoroscopic flexion-extension recording \\
\hline GRADE & Grading of Recommendations Assessment, Development and Evaluation \\
\hline $\mathrm{HC}$ & healthy control \\
\hline IDE & investigational device exemption \\
\hline MD & mean difference \\
\hline MECIR & Methodological Expectations for Cochrane Intervention Reviews \\
\hline MEDLINE & Medical literature analysis and retrieval system online \\
\hline METC & Medical ethical committee \\
\hline MRI & magnetic resonance imaging \\
\hline MRC & medical research council \\
\hline NDI & neck disability index \\
\hline NGF & normalized gradient field \\
\hline NRS & numerical rating scale \\
\hline NSAID & non-steroidal anti-inflammatory drug \\
\hline RCT & randomized controlled trial \\
\hline RR & relative risk \\
\hline $\mathrm{ROB}$ & risk of bias \\
\hline SD & standard deviation \\
\hline & Supplemental Digital Content \\
\hline
\end{tabular}


SF-36-MCS

Short Form-36 mental component summary

SF-36-PCS Short Form-36 physical component summary

SIGLE System for Information on Grey Literature

SMD standardised mean difference

SROM segmental range of motion

VAS

visual analogue scale 


\section{Dankwoord}


In de afgelopen jaren heb ik mij gelukkig mogen prijzen met vele vrienden en familie die me hebben gesteund bij het schrijven van dit proefschrift, maar belangrijker nog, bij het leven daaromheen.

Velen van jullie heb ik de afgelopen jaren veel te weinig gezien, ik hoop dat de komende tijd te kunnen inhalen. Onderstaande lijst met personen is niet uitputtend, ik ben met aan zekerheid grenzende waarschijnlijkheid mensen vergeten te vermelden.

Prof. dr. de Bie, beste Rob, dank voor je begeleiding de afgelopen jaren. Je nuchtere kijk op onze manuscripten, 'uitdagingen' in het onderzoek, en 'het systeem' werkt relativerend. Je kritische blik op Engelstalige manuscripten, en analytische kijk op de inhoud ervan was zeer welkom. Ook de logistieke ondersteuning, mede via je rechter hand Conny, was onmisbaar. Dank voor je begeleiding al deze jaren.

Dr. van Santbrink, beste Henk, dank voor alle tijd en moeite die je als co-promotor in dit onderzoek hebt gestoken. Ik was pas net als ANIOS aan het werk toen je vroeg of ik interesse had in een onderzoekstraject. Dat onderzoek is in de jaren erna uitgekristalliseerd tot dit proefschrift. Naast leerzaam waren de congressen die we bezochten ook gezellig, en hebben we altijd tijd gevonden om ook de stad even te verkennen en een (liefst lokaal) biertje te drinken. Daarnaast dank voor je rol als plaatsvervangend opleider/opleider. Ik heb veel van je geleerd, je kalme manier om iemand 'door een ingreep heen te praten' speelde daarbij een grote rol. Ik hoop de komende jaren nog veel van je te leren.

Prof. dr. van Mameren, beste Henk, je bent sterk verbonden met het onderwerp, je hebt in je eigen proefschrift ooit segmentale volgorde van bewegen in het leven geroepen als uitkomstmaat. We hebben vaak bij jou en Sylvia aan de keukentafel zitten overleggen met stapels grafieken om ons heen (en een Cuvée du Château). Hierbij werden pittige discussies niet geschuwd, waarbij je pas te overtuigen was (en bent) met harde data. Ik denk dat niemand kritischer is dan jij bij het beschrijven van methoden en uitkomsten, en hoe frustrerend dat soms ook kan zijn, het heeft de publicaties sterker gemaakt. Op naar nieuwe projecten.

Graag zou ik de leden van de beoordelingscommissie, bestaande uit prof. dr. L. van Rhijn, dr. L. Jacobi-Postma, prof. dr. W. Peul, prof. dr. Y. Temel, en dr. A. Vilanova, willen bedanken voor de beoordeling van dit proefschrift.

Beste collega neurochirurgen van de afdeling neurochirurgie Maastricht, dank voor de opleiding die ik heb mogen genieten in ons centrum. Ik ben erg blij dat ik na het afronden van mijn opleiding zo warm verwelkomd ben in de staf, ondanks de roerige tijden waarin de afdeling zich bevond. Ik zie een mooie toekomst voor de neurochirurgie in Academisch Neurochirurgisch Centrum Limburg.

Jasper en Yasin, aan jullie ben ik een apart dankwoord verschuldigd. Via jullie twee ben ik ooit de neurochirurgie ingerold, nadat ik als tweedejaars student geneeskunde interesse toonde in onderzoek binnen het vak. De rest is geschiedenis. Punt. 
In de afgelopen jaren heb ik mij gelukkig mogen prijzen met fijne collega's in de assistentengroep, sommigen daarvan zijn in het vorige paragraaf al aan bod gekomen. Vincent, je bent weliswaar geen assistent meer maar we hebben onze opleiding grotendeels samen afgelegd nadat ik terug kwam uit mijn onderzoeksperiode. Het was erg prettig om samen te kunnen sparren over de opleiding, het werk, en alles daaromheen. Youssef, Roel, Felix, Anouk, Tim, Suzanne, Karlijn, Jeroen, en Rick, dank voor de collegialiteit en de goede werksfeer de afgelopen jaren. Succes met jullie carrières binnen en buiten de neurochirurgie, en binnen en buiten Maastricht. Luc, je werkt nu al jaren als PA binnen onze afdeling, en hebt inmiddels menig A(N)IOS ingewerkt op de afdeling. Ook heb je je altijd ingezet voor het begeleiden van de patiënten die deelnamen aan de RCT. Dank voor je inzet en je altijd gepaste humor op de afdeling.

Angélique, Carla, Charlotte, Claudia, Regina, Monique, Monique en Nicole, dank voor de secretariële ondersteuning, het meedenken, en de flexibiliteit als zich weer een verandering in het rooster of OK-programma voordoet.

Drs. Curfs, drs. Van den Boogaart, dr. Willems, drs. Tilman, en dr. Van Hemert, beste Inez, Mark, Paul, Pieter en Wouter, dank voor de fijne samenwerking binnen de gecombineerde orthopedische/neurochirurgische poli's en OK's. Ik hoop nog veel met jullie samen te werken en van jullie te leren de komende tijd.

Rianne en Bram, jullie zijn voor de master thesis van Rianne vanuit de TU/e met de beeldherkenningssoftware aan de slag gegaan en hebben daarvoor een heel mooi algoritme ontwikkeld dat absoluut zijn meerwaarde heeft bewezen. Het was een stuk moeilijker geweest om dit onderzoek te voltooien als alle contouren met de hand gevolgd hadden moeten worden (zoals vroeger). Dank voor jullie hulp.

Dionne, Claire en Charles, dank voor jullie ondersteuning bij het maken van de röntgendoorlichtingen van alle klachtenvrije proefpersonen en de patiënten die aan de RCT deelnamen.

Hamid, Inge, en Rick, ik heb jullie in de afgelopen jaren mogen begeleiden tijdens semiarts stages en keuzeblokken, en daarnaast bij onderzoeksprojecten. Dank voor jullie harde werk. Veel succes in jullie verdere carrières. Sayf en Suzanne, ik heb mogen meewerken aan jullie onderzoeksprojecten naar de axiale rotatiecomponent bij scoliose, en door jullie harde werk zijn daar twee mooie publicaties uit voortgevloeid. Dank voor jullie harde werk en jullie enthousiasme.

Ik kijk ook terug op een mooie periode op het orthopedisch research lab. Tim, Pieter en Chris, dank voor het faciliteren van mijn plekje daar. Don, Andy, Jim, Laura, Ilona, Guus, Alex, en Liesbeth het was gezellig op congres, met de Cake van de Week, het Bier van de Maand, de Koffie van de Dag, en belangrijke projecten als de 'Tostifieerbaarheidsfactor'. 
Annerie, Bart, Elles, Hanneke, Inge, Jeroen, en Steven, we hebben de studie geneeskunde samen mogen volbrengen. Het was gezellig om samen te studeren, een wijntje te drinken, surprises te maken en krijgen met Sinterklaas, samen te eten in het Huis der Zeven Schimmelen, weekendjes weg te gaan in huurhuisjes van mensen die al dan niet een kikker-fetisj hadden, en te klagen over het Portfolio systeem (als dit onzalige systeem reeds in de tijd van Dante bedacht was, had hij zeker een $8^{\text {ste }}$ laag binnen een niet nader te noemen bijzonder warme omgeving beschreven). We zien elkaar helaas niet meer zo vaak als destijds, maar het blijft gezellig om jullie weer te zien als de kans zich weer eens voordoet. Das Glas darf nicht leer sein! B, J, en Steef, ik kijk alweer uit naar onze volgende LAN-party.

Steven, als onderdeel van bovenstaande groep verdien je toch een speciaal bedankje. We hebben ook na onze opleiding nog goed contact gehouden, zijn vrijwel jaarlijks gaan skiën, zijn regelmatig gaan hardlopen, hebben uren en uren en uren (en dagen) Talisman en andere bordspellen gespeeld met jou en Odette onder het genot van een paar wijntjes. Als het echt een goede avond was (voor iedereen behalve jou) eindigde de avond met Cointreau $(-)$. Ik vind het ergens erg jammer dat jij en Odette met de jongens in Zwitserland zijn gaan wonen en werken, hoewel het wel fantastisch is om op een steenworp afstand te kunnen skiën bij een bezoekje. Bedankt dat je mijn paranimf wilt zijn.

Nard, Sjoerd en Tom (a.k.a. de 'Masters'), we kennen elkaar nu al meer dan 20 jaar. In die tijd hebben we veel plezier gehad met stappen, met het bezoeken van festivals, en met dwaze projecten (waarbij in de meerderheid van de gevallen weinig tot geen schade aan koeien, weilanden, afrasteringen, auto's en onszelf aangericht is). Daarnaast vele avonden met een filmpje, een goed gesprek, en een biertje, waarbij de kwaliteit van het bier in de loop der jaren wat toenam (misschien wel in tegenstelling tot de kwaliteit van de films). Dank voor jullie vriendschap al die jaren. Nard, bedankt dat jij namens deze drieeenheid mijn paranimf wilt zijn.

Lindi, dank voor het ontwerpen van de mooie kaft. Je grafische kwaliteiten hebben al eerder een proefschrift mogen sieren en ik ben blij dat je ook deze uitdaging hebt aangenomen. Bovenal dank voor je vriendschap door de jaren.

Lieve pap en mam, Peer en Marianne, dank voor het bieden van een liefdevol thuis en jullie onvoorwaardelijke steun de afgelopen decennia. Ik was niet helemaal gepland en jullie hebben het niet altijd gemakkelijk gehad met het combineren van het afronden van studies, werk, en een oplopend aantal kinderen (en kleinkinderen). Ik ben trots op jullie beiden voor wat jullie bereikt hebben, jullie hebben me geleerd om door te zetten. Bovenal zijn jullie beiden een fantastisch voorbeeld als mens. Ik ben ontzettend dankbaar dat ik jullie zoon ben.

Lieske, Joep, Frans, Godelieve en Tof, lieve broertjes en zusjes, ik ben dankbaar voor het feit dat ik jullie grote broer mag zijn, en trots op jullie allemaal. Ook jullie heb ik de afgelopen jaren minder vaak gezien dan ik zou willen, ik hoop dat we dat de komende tijd 
kunnen inhalen. Als voltallig gezin recent een obstacle run doen is een mooi begin geweest.

Anneke, Jac, Jan en Patricia, Renske en Nick, bedankt dat jullie me zo hartelijk hebben opgenomen in de familie. Anneke, dank voor de tijd die je doorbrengt met de kleinkinderen. Owen is altijd blij als oma weer langskomt. PS! is dit nu het boekje waar de zegeltjes in horen?

Lieve Marjolein, zonder jouw steun was dit proefschrift waarschijnlijk niet afgerond. Je hebt me al die jaren gesteund, gestimuleerd door te zetten op de momenten dat het tegenzat, meegedacht over manuscripten, problemen besproken en je heldere geest en analytische kijk hebben daarbij veel geholpen. We hebben elkaar ontmoet in het eerste jaar dat ik als promovendus op het orthopedisch research lab te gast mocht zijn (in de wandelgangen het weeskindje van de neurochirurgie). Nu ben je al enkele jaren zelf gepromoveerd, en op datzelfde lab zelfs opgeklommen tot UD terwijl we eigenlijk ernaar streefden om tegelijk te promoveren. Ik zeg het veel te weinig, maar ik houd nog steeds zielsveel van je en ben blij en dankbaar dat jij mijn vrouw bent. Het meest waardevolle dat ik overhoud aan dit promotietraject zijn jij en de kinderen.

Lieve Owen en Lisa, zonder jullie was dit proefschrift waarschijnlijk 2 jaar eerder klaar geweest, en dat is het meer dan waard geweest. Elke dag dat ik jullie mag zien opgroeien is een geschenk waarvoor woorden tekort schieten. 

Curriculum Vitae 


\section{Nederlands}

Toon Boselie werd in Heerlen geboren op 22 januari 1981. Hij ging naar basisschool Nutsschool Oost te Maastricht en in verband met een verhuizing later naar basisschool Windekind te Heerlen. In 1993 vervolgde hij zijn schoolcarrière op het gymnasium van het Bernardinuscollege te Heerlen. Halverwege het eerste jaar verhuisde het gezin opnieuw en vervolgde hij zijn scholing op het gymnasium van R.K.S.G. Serviam te Sittard waar hij zijn diploma in 1999 haalde. Hij ging daarna Biomedische Technologie studeren aan de TU in Eindhoven, maar stopte daar na een jaar mee om geneeskunde te gaan studeren. Helaas werd hij uitgeloot waarop hij een Staatexamen VWO heeft behaald om een hogere lotingsgroep te bereiken. Hij werd daarop ingeloot en startte in 2001 met de opleiding geneeskunde aan de Universiteit Maastricht. In het tweede jaar van de opleiding werd zijn interesse voor de neurochirurgie gewekt door een keuzeblok. In 2005 behaalde hij zijn doctoraal cum laude. Hij sloot zijn opleiding af met een participatiestage in de neurochirurgie en behaalde in 2007 zijn artsexamen. Daarop startte hij na sollicitatie bij prof. dr. E. Beuls, het toenmalig afdelingshoofd, als ANIOS neurochirurgie. Hij werkte twee jaar als ANIOS en startte ondertussen met een promotietraject onder begeleiding van $\mathrm{dr}$. $\mathrm{H}$. van Santbrink, prof. dr. H. van Mameren en prof. dr. R. de Bie. Nadat een aanvraag voor financiering in de vorm van een profileringsfonds van het MUMC+ toegekend werd, kon hij in 2009 voor drie jaar fulltime onderzoek doen. In diezelfde periode werd hem na een positief gesprek met prof. dr. K. van Overbeeke, het toenmalig afdelingshoofd van de neurochirurgie, een opleidingsplaats aangeboden aansluitend aan de onderzoeksfase. In mei 2012 begon hij met de opleiding tot neurochirurg. Gedurende de laatste jaren van de opleiding heeft hij zich meer en meer gedifferentieerd richting de wervelkolomchirurgie. In de laatste fase van zijn opleiding heeft hij een observership in de Cleveland Clinic (Cleveland, Ohio, VS) verricht onder begeleiding van prof. dr. E. Benzel. Hij heeft in oktober 2017 zijn opleiding tot neurochirurg afgerond en is sindsdien werkzaam als staflid in het MUMC+ en het Zuyderland MC te Heerlen/Sittard-Geleen. Zijn primaire aandachtsgebied is daarbij de wervelkolomchirurgie. 


\section{English}

Toon Boselie was born in Heerlen on January 22, 1981. He went to primary school at Nutsschool Oost in Maastricht, followed by Windekind in Heerlen due to moving. He started grammar school at the Bernardinuscollege in Heerlen, followed by R.K.S.G. Serviam in Sittard due to moving again. He graduated from grammar school in 1999. He then studied Biomedical Engineering at the Technical University Eindhoven for a year, after which he decided to study Medicine. He was not selected in the draw for the restrictedintake study in 2000, and decided to retake his grammar school exams for a higher chance in the next draw. He was selected in 2001, and started studying Medicine at Maastricht University. His interest for neurosurgery was kindled in the second year due to an elective. In 2005 he received his Bachelor degree with honors. After doing an elective in neurosurgery in the last year of the study he graduated in 2007. The worked as a residentnot-in-training at the department of neurosurgery in Maastricht for two years. During this period he started a PhD-trajectory, under the supervision of dr. H. van Santbrink, prof. dr. $H$. van Mameren and prof. dr. R. de Bie. Due to a grant he received from the hospital he was given the opportunity to plan almost three years of fulltime research. Prof. dr. K. van Overbeeke, the head of the department at that time, offered him a place as a neurosurgeon in training after this research period. He started his residencies in May 2012. During the last years of his residencies he became focused on spinal surgery more and more. In the last phase of his residencies he visited the department of neurological surgery of the Cleveland Clinic (Cleveland, Ohio, VS), under the supervision of prof. dr. E. Benzel. He finished his training October 2017 and started as a neurosurgeon at Maastricht University Medical Center and Zuyderland MC Heerlen/Sittard-Geleen in November 2017. His primary field of interest is spine surgery. 



\section{List of Publications}

van Aalst J, Beuls EA, Cornips EM, van Straaten HW, Boselie AF, Rijkers K, Weber JW, Vles JS. The spinal dermal-sinus-like stalk. Childs Nerv Syst. 2009 Feb; 25(2):191-7.

Reinartz R, Platel B, Boselie T, van Mameren H, van Santbrink H, Romeny BH. Cervical vertebrae tracking in video-fluoroscopy using the normalized gradient field. Med Image Comput Comput Assist Interv. 2009; 12(Pt 1):524-31.

van Aalst J, Boselie TF, Beuls EA, Vles JS, van Straaten HW. Spinal congenital dermal sinus in a chick embryo model. Laboratory investigation. J Neurosurg Pediatr. 2009 Jan; 3(1):248.

Boselie TF, van Santbrink H. Letter to the article of Jiang et al. Arch Orthop Trauma Surg. 2012 Feb; 132(2):153.

Boselie TF, Willems PC, van Mameren H, de Bie R, Benzel EC, van Santbrink H. Arthroplasty versus fusion in single-level cervical degenerative disc disease (Withdrawn). Cochrane Database Syst Rev. 2012 Sep 12; 9: CD009173.

Boselie TF, Willems PC, van Mameren H, de Bie R, Benzel EC, van Santbrink H. Arthroplasty versus fusion in single-level cervical degenerative disc disease: a Cochrane review. Spine (Phila Pa 1976). 2013 Aug 1; 38(17):E1096-107.

Boselie TF, van Mameren H, de Bie RA, van Santbrink H. Cervical spine kinematics after anterior cervical discectomy with or without implantation of a mobile cervical disc prosthesis; an RCT. BMC Musculoskelet Disord. 2015 Feb 21; 16:34.

Eijgenraam SM, Boselie TF, Sieben JM, Bastiaenen CH, Willems PC, Arts JJ, Lataster A. Development and assessment of a digital $\mathrm{X}$-ray software tool to determine vertebral rotation in adolescent idiopathic scoliosis. Spine J. 2017 Feb; 17(2):260-265.

Boselie TF, Willems PC, van Mameren $\mathrm{H}$, de Bie R, van Santbrink H. Arthroplasty versus fusion in single level cervical degenerative disc disease (protocol for update) Cochrane Database Syst Rev. 2016

Boselie TF, van Santbrink H. Arthroplasty in cervical degenerative disc disease: fulfilling its long-term promise? (Editorial). J Spine Surg. 2016 Dec; 2(4):359-361.

Boselie TFM, van Santbrink H, de Bie RA, van Mameren H. A pilot study of sequence of segmental contributions in the lower cervical spine during active extension and flexion: healthy controls vs. cervical degenerative disc disease patients. Spine (Phila Pa 1976). 2017 Jun 1;42 (11):E642-E647. 
Faraj SSA, Boselie TFM, Vila-Casademunt A, de Kleuver M, Holewijn RM, Obeid I, Acaroglu E, Alanay A, Kleinstück F, Pérez-Grueso FS, Pellisé F; European Spine Study Group. Radiographic Axial Malalignment is Associated With Pretreatment Patient-Reported Health-Related Quality of Life Measures in Adult Degenerative Scoliosis: Implementation of a Novel Radiographic Software Tool. Spine Deform. 2018 Nov - Dec; 6(6):745-752.

Boselie TFM, de Bie RA, van Mameren H, van Santbrink H. Sequence of segmental contributions in the caudal cervical spine in arthroplasty and ACD patients: a randomized controlled trial.

(Submitted)

M. Pishnamaz, I. Curfs, D. Uhing, C. Herren, H. van Santbrink, C.A. Mueller, M. Scholz, P. Lichte, K. Rijkers, T. Boselie, F. Hildebrand, P. Willems, P. Kobbe. Two-nation comparison of classification and treatment of subaxial cervical spine fractures: An internet-based multicenter study among spine surgeons. World Neurosurgery, 2018 (E-pub ahead of print) 


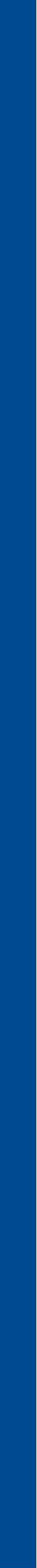

